\title{
The sensitivity of radiotherapy to tissue composition and its estimation using novel dual energy $\mathrm{CT}$ methods
}

Citation for published version (APA):

Landry, G. (2014). The sensitivity of radiotherapy to tissue composition and its estimation using novel dual energy CT methods. [Doctoral Thesis, Maastricht University]. Datawyse / Universitaire Pers Maastricht. https://doi.org/10.26481/dis.20140612gl

Document status and date:

Published: 01/01/2014

DOI:

10.26481/dis.20140612gl

Document Version:

Publisher's PDF, also known as Version of record

\section{Please check the document version of this publication:}

- A submitted manuscript is the version of the article upon submission and before peer-review. There can be important differences between the submitted version and the official published version of record.

People interested in the research are advised to contact the author for the final version of the publication, or visit the DOI to the publisher's website.

- The final author version and the galley proof are versions of the publication after peer review.

- The final published version features the final layout of the paper including the volume, issue and page numbers.

Link to publication

\footnotetext{
General rights rights.

- You may freely distribute the URL identifying the publication in the public portal. please follow below link for the End User Agreement:

www.umlib.nl/taverne-license

Take down policy

If you believe that this document breaches copyright please contact us at:

repository@maastrichtuniversity.nl

providing details and we will investigate your claim.
}

Copyright and moral rights for the publications made accessible in the public portal are retained by the authors and/or other copyright owners and it is a condition of accessing publications that users recognise and abide by the legal requirements associated with these

- Users may download and print one copy of any publication from the public portal for the purpose of private study or research.

- You may not further distribute the material or use it for any profit-making activity or commercial gain

If the publication is distributed under the terms of Article $25 \mathrm{fa}$ of the Dutch Copyright Act, indicated by the "Taverne" license above, 


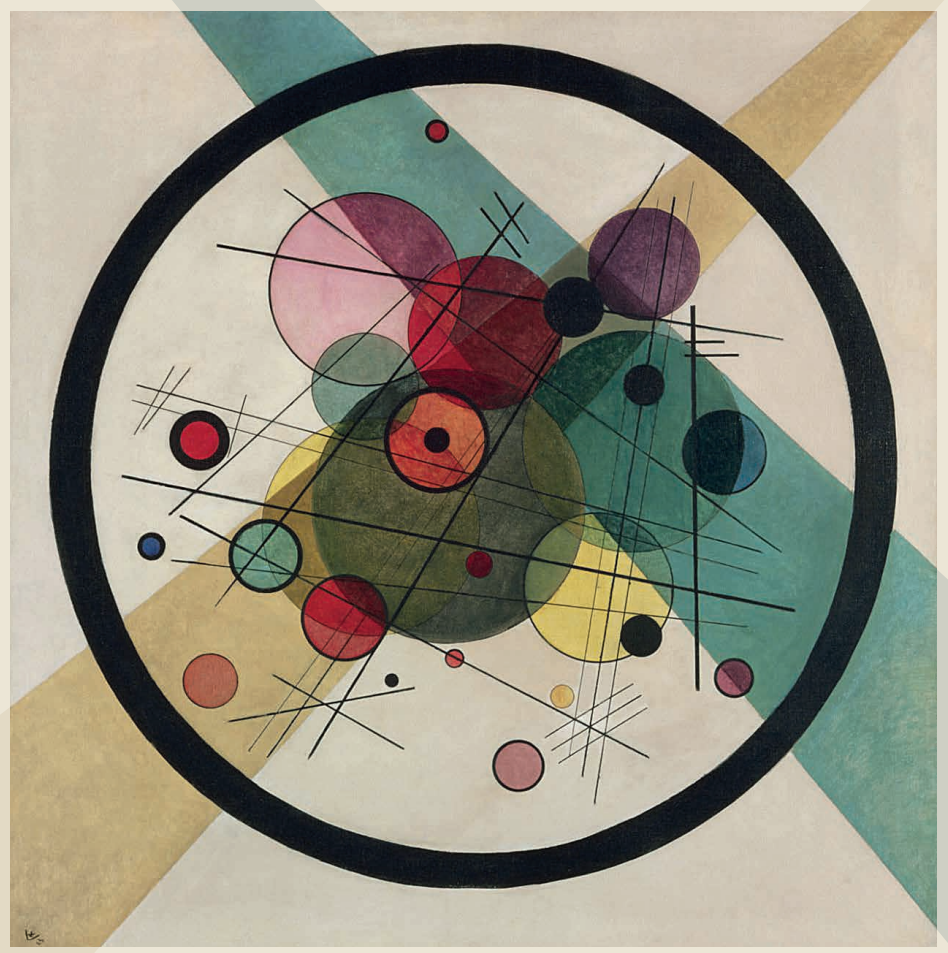

THE SENSITIVITY OF RADIOTHERAPY TO TISUUE COMPOSITION AND ITS ESTIMATION UIING NOVEL DUAL ENERGY (T METHODS

Guillaume Landry 


\section{Cover illustration}

\section{Circles in a Circle}

Vasily Kandinsky, Oil on canvas, 1923

$387 / 8 \times 375 / 8$ inches $(98.7 \times 95.6 \mathrm{~cm})$

Philadelphia Museum of Art: The Louise and Walter Arensberg Collection, 1950

(C) VG Bild-Kunst, Bonn, Germany

\section{Production}

Printing: Datawyse | Universitaire Pers Maastricht

ISBN: $\quad 9789461593290$

(C) Copyright G Landry, Maastricht 2014 


\section{The sensitivity of radiotherapy to tissue composition and its estimation using novel dual energy CT methods}

\section{Thesis}

to obtain the degree of Doctor at

Maastricht University,

on the authority of the Rector Magnificus, Prof. dr. L.L.G. Soete

in accordance with the decision of the Board of Deans,

to be defended in public

on Thursday 12 June 2014, at 12:00

by

Guillaume Landry

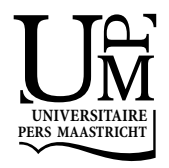




\section{Supervisor:}

Prof. Dr. F. Verhaegen

\section{Co-supervisor:}

Prof. Dr. L. Beaulieu, Universite Laval / CHUQ

\section{Assessment Committee:}

Prof. Dr. P. Lambin (chairman)

Dr. Å. Carlsson Tedgren, Linköping University

Prof Dr. A. Nahum, Liverpool Cancer Research Centre

Prof. Dr. D. De Ruysscher, UZ Leuven

Dr. J.L.M. Venselaar, Instituut Verbeeten 


\section{Contents}

$\begin{array}{lll}\text { Chapter } 1 & \text { Introduction and outline of the thesis } & 7\end{array}$

Chapter 2 Review of Monte Carlo methods in brachytherapy 13

Chapter 3 Sensitivity of low energy brachytherapy Monte Carlo dose calcula- 29 tions to uncertainties in human tissue composition

Chapter 4 The difference of scoring dose to water or tissues in Monte Carlo dose calculations for low energy brachytherapy photon sources

Chapter 5 Simulation study on potential accuracy gains from dual energy CT tissue segmentation for low-energy brachytherapy Monte Carlo dose calculations

Chapter 6 Extracting atomic numbers and electron densities from a dual source dual energy CT scanner: experiments and a simulation model

Chapter 7 Systematic evaluation of noise levels from dual energy acquisitions with a dual source CT scanner: comparing iterative and analytical reconstruction methods

Chapter 8 Deriving effective atomic numbers from DECT based on a parameterization of the ratio of high and low linear attenuation coefficients

Chapter 9 Deriving concentrations of oxygen and carbon in human tissues using single- and dual-energy CT for ion therapy applications

Chapter 10 General discussion and future perspectives

Summary

List of publications

Acknowledgements

Curriculum vitae 



\section{CHAPTER}

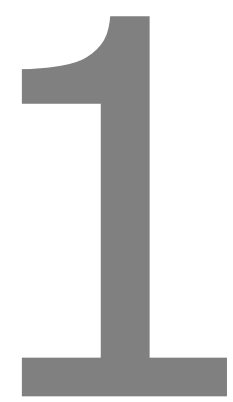

Introduction and outline of the thesis 



\section{INTRODUCTION}

Low dose rate (LDR) transperineal permanent seed implantation performed under transrectal ultrasound (TRUS) guidance is widely used in the treatment of early stage

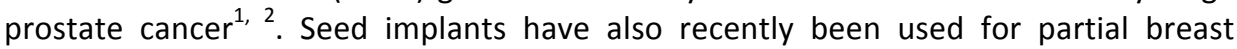
irradiation of early stage breast cancer patients ${ }^{3-5}$ following breast-conserving surgery. Both these techniques employ low energy photons (energies below $50 \mathrm{keV}$ ) to irradiate malignancies.

The dosimetry of low energy photon brachytherapy procedures based on LDR seed implants using ${ }^{125} \mathrm{I}$ and ${ }^{103} \mathrm{Pd}$ or high dose rate (HDR) electronic brachytherapy sources (EBS) follows the AAPM Radiation Therapy Committee Task Group 43 formalism $^{6,7}$ (TG-43). The use of dosimetric functions obtained in uniform water spheres of $15 \mathrm{~cm}$ radius has reported shortcomings, such as the inability to handle tissue heterogeneities and interseed attenuation in prostate and breast seed implants ${ }^{8-11}$. These shortcomings are further discussed in chapter 2.

The AAPM Radiation Therapy Committee Task Group 186 (TG-186) ${ }^{12}$ has recently published their investigations on model based dose calculation (MBDC) algorithms capable of addressing the shortcomings of TG-43. Monte Carlo (MC) simulations have been recommended as one of the alternatives for their ability to accurately transport low energy photons in realistic geometries ${ }^{10}$. To achieve maximum accuracy in this energy range, MC methods and other model-based algorithms require voxel by voxel knowledge of mass density $\rho$ and tissue elemental composition.

In current clinical practice, quantitative information on tissues is determined by conventional single energy CT (SECT) in the form of attenuation coefficients (often expressed by Hounsfield Units, HU). From these, mass or electron densities are derived using empirical relationships. The current standard for heterogeneous dose calculations, which are almost exclusively used in external beam therapy, is based on these densities and does not account for tissue type ${ }^{13}$. Tissue type identification based on SECT measurement of the linear attenuation coefficient has been shown to lead to MC dose calculation errors in the megavoltage photon and electron energy range $\mathrm{e}^{14}$. Because of the strong dependence of the photoelectric cross sections on the tissue's atomic number, such errors are expected to be amplified in the low energy range employed in brachytherapy.

\section{Objective of the study}

The goal of this study was twofold. In the first part of the study MC techniques were employed to assess the sensitivity of LDR brachytherapy dosimetry using low energy sources $(<50 \mathrm{keV})$ to variations in human tissue composition. Dose distributions were calculated for a series of human tissues and the impact of uncertainties on the composition of these tissues were quantified. In the second part of the study a dual energy CT (DECT) procedure for tissue composition assignment was developed and 
compared to the conventional tissue composition assignment scheme based on SECT imaging. DECT imaging consists of the acquisition of two CT images taken at different $k V p$ and exploits the $Z$ dependence of photon attenuation to extract additional information on tissue composition, generally in the form of a single effective atomic number $Z_{\text {eff. }}$. The DECT technique was first validated then applied to the case of low energy brachytherapy dose calculations. Furthermore the DECT method was improved in terms of accuracy and precision, and its applicability to other areas of radiotherapy investigated.

\section{Thesis outline}

Chapter 1 covers the objective of the study and introduces the issue of tissue composition assignment for LDR brachytherapy based on low energy sources. Chapter 2 reviews the use of $\mathrm{MC}$ methods in brachytherapy. In that chapter it is presented why MC methods are essential in accounting for patient inhomogeneities as well as the issues associated with CT imaging and tissue composition assignment. In Chapter $\mathbf{3}$ the sensitivity of brachytherapy dosimetry for low energy sources to variations in human tissue compositions around population averaged values is investigated for a series of tissues relevant for brachytherapy. Chapter 4 covers the difference between calculating the radiation dose deposited in water or tissues for photons transported in various tissues. Chapter $\mathbf{5}$ is the first chapter dedicated to dual energy CT. In that chapter a simulation tool for CT imaging is employed to compare conventional SECT imaging and DECT imaging in the context of low energy brachytherapy dose calculations. In Chapter 6 the simulation tool is validated against measurements obtained from a DECT scanner. The next two chapters deal with improvements of the DECT method in terms of precision and accuracy. Chapter $\mathbf{7}$ is dedicated to the evaluation of a commercial noise reduction algorithm for CT imaging in the context of DECT while Chapter 8 presents a novel, simpler and more accurate way of deriving $Z_{\text {eff. }}$ Chapter 9 aims at evaluating whether DECT can be useful for deriving the concentrations of carbon an oxygen in human tissues for ion therapy applications. Finally Chapter $\mathbf{1 0}$ contains an outlook on the use of DECT in brachytherapy and radiotherapy in general. Chapters 3-9 are based on papers which are either published or under review in international peer-reviewed journals. Chapter 2 is an abbreviated version of a book chapter.

\section{REFERENCES}

${ }^{1}$ H.H. Holm and J. Gammelgaard, "Ultrasonically guided precise needle placement in the prostate and the seminal vesicles," J Urol 125, 385-387 (1981).

${ }^{2}$ H.H. Holm, N. Juul, J.F. Pedersen, H. Hansen, and I. Stroyer, "Transperineal 125iodine seed implantation in prostatic cancer guided by transrectal ultrasonography," J Urol 130, 283286 (1983).

3.P. Pignol, B. Keller, E. Rakovitch, R. Sankreacha, H. Easton, and W. Que, "First report of a permanent breast 103Pd seed implant as adjuvant radiation treatment for early-stage breast cancer," Int J Radiat Oncol Biol Phys 64, 176-181 (2006). 
${ }^{4}$ B. Keller, R. Sankreacha, E. Rakovitch, P. O'Brien, and J.P. Pignol, "A permanent breast seed implant as partial breast radiation therapy for early-stage patients: a comparison of palladium-103 and iodine-125 isotopes based on radiation safety considerations," Int J Radiat Oncol Biol Phys 62, 358-365 (2005).

${ }^{5}$ J.P. Pignol, E. Rakovitch, B.M. Keller, R. Sankreacha, and C. Chartier, "Tolerance and acceptance results of a palladium-103 permanent breast seed implant Phase I/II study," Int J Radiat Oncol Biol Phys 73, 1482-1488 (2009).

${ }^{6}$ R. Nath, L.L. Anderson, G. Luxton, K.A. Weaver, J.F. Williamson, and A.S. Meigooni, "Dosimetry of interstitial brachytherapy sources: recommendations of the AAPM Radiation Therapy Committee Task Group No. 43. American Association of Physicists in Medicine," Med Phys 22, 209-234 (1995).

${ }^{7}$ M.J. Rivard, et al., "Update of AAPM Task Group No. 43 Report: A revised AAPM protocol for brachytherapy dose calculations," Med Phys 31, 633-674 (2004).

${ }^{8}$ H. Afsharpour, J.-P. Pignol, B. Keller, J.-F. Carrier, B. Reniers, F. Verhaegen, and L. Beaulieu, "Influence of breast composition and interseed attenuation in dose calculations for postimplant assessment of permanent breast ${ }^{103}$ Pd seed implant," Phys Med Biol 55, 4547-4561 (2010).

${ }^{9}$ G.S. Burns and D.E. Raeside, "The accuracy of single-seed dose superposition for I-125 implants," Med Phys 16, 627-631 (1989).

${ }^{10}$ J.F. Carrier, M. D'Amours, F. Verhaegen, B. Reniers, A.G. Martin, E. Vigneault, and L. Beaulieu, "Postimplant dosimetry using a Monte Carlo dose calculation engine: a new clinical standard," Int J Radiat Oncol Biol Phys 68, 1190-1198 (2007).

${ }^{11}$ O. Chibani, J.F. Williamson, and D. Todor, "Dosimetric effects of seed anisotropy and interseed attenuation for 103Pd and 125I prostate implants," Med Phys 32, 2557-2566 (2005).

${ }^{12}$ L. Beaulieu, et al., "Report of the Task Group 186 on model-based dose calculation methods in brachytherapy beyond the TG-43 formalism: Current status and recommendations for clinical implementation," Med Phys 39, 6208-6236 (2012).

${ }^{13} \mathrm{~N}$. Papanikolaou, et al., "AAPM Report no. 85: Tissue inhomogeneity corrections for megavoltage photon beams. ," (2004).

${ }^{14} \mathrm{~F}$. Verhaegen and S. Devic, "Sensitivity study for CT image use in Monte Carlo treatment planning," Phys Med Biol 50, 937-946 (2005). 



\section{CHAPTER}

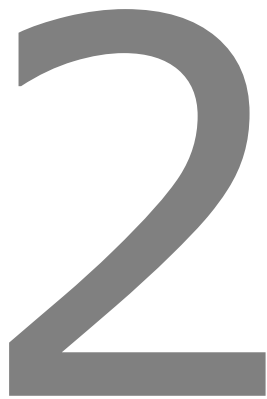

Review of Monte Carlo methods in brachytherapy

Guillaume Landry, Mark J. Rivard, Jeffrey F. Williamson, Frank Verhaegen Book chapter from:

F. Verhaegen and J. Seco, Monte Carlo techniques in radiation therapy, Taylor \& Francis, 2013 



\section{INTRODUCTION}

As in other branches of radiation therapy, Monte Carlo (MC) simulation has become an essential dosimetry tool in modern brachytherapy, playing key roles in both clinical practice and research. The most established application of $M C$ methods in brachytherapy is the determination of dose rate distributions around individual radiation sources. Modern sources generally contain low energy radionuclides such as ${ }^{103} \mathrm{Pd}$, ${ }^{125} \mathrm{I}$, or ${ }^{131} \mathrm{Cs}$ (mean energies < $0.05 \mathrm{MeV}$, referred to henceforth as low-energy sources); higher energy radionuclides such as ${ }^{192} \mathrm{Ir}$ and ${ }^{137} \mathrm{Cs}$ (mean energies 0.355 and $0.662 \mathrm{MeV}$ ); or may consist of miniature $\mathrm{x}$-ray sources (e.g. $50 \mathrm{kVp}$ Bremsstrahlung spectrum). Both source geometries and clinical applications are quite variable: in low dose rate (LDR) brachytherapy, radioactive material and radio-opaque markers are encapsulated to form permanently implantable seeds; while in high dose rate (HDR) brachytherapy e.g. an iridium pellet is welded to the tip of a single-stepping source remote-afterloader cable. Miniature x-ray sources with tungsten anodes also fall in the HDR category, even though they emit photons in the low energy range. While inversesquare law dependence is the dominating feature of brachytherapy dose distributions, photon attenuation and scatter build-up in the surrounding medium and radiation interactions within the source structure give rise to anisotropic dose distributions. The significant modulation of dose distributions must be properly modeled to attain clinically acceptable dosimetric accuracy, and these features are not readily derived from analytical methods such as the Sievert integral ${ }^{1}$. The complexities of experimentally measuring single-source dose distributions caused by the sharp dose gradients, low photon energies and dose rates associated with brachytherapy make computational dosimetry techniques such as MC simulations an essential tool in brachytherapy ${ }^{2}$.

The first computational efforts towards obtaining brachytherapy dose distributions are attributed to the 1960s work of Meisberger who derived 1D tissue-attenuation and scatter build-up factors for ${ }^{198} \mathrm{Au},{ }^{192} \mathrm{Ir},{ }^{137} \mathrm{Cs},{ }^{226} \mathrm{Ra}$, and ${ }^{60} \mathrm{Co}$ point sources ${ }^{3}$, while Dale was the first to apply similar techniques to modern ${ }^{125}$ I in $1983^{4}$. Although MC modeling of a 3D brachytherapy source geometry was performed as early as 1971 by Krishnaswamy for ${ }^{252} \mathrm{Cf}$ needles ${ }^{5}$, it took another decade for the field to fully embrace 3D modeling. Williamson showed in 1983, using 3D MC simulations, that the Sievert integral deviated by 5\%-100\% from MC results for mono-energetic photons of energies lower than $0.3 \mathrm{MeV}$ emitted from an encapsulated line source, emphasizing the need for accurate computational dosimetry ${ }^{6}$. Burns and Raeside were the first to fully model a commercial ${ }^{125}$ I seed (model 6711, 3M, now GE HealthCare / Oncura), modeling the silver radiomarker, ${ }^{125}$ I distribution and titanium encapsulation to obtain a $2 \mathrm{D}$ dose rate distribution ${ }^{7}$. Since the range in water of secondary electrons generated by $30 \mathrm{keV}$ photons is less than $20 \mu \mathrm{m}$, Burns and Raeside did not transport electrons in their simulations and scored collision kerma using a track-length estimator ${ }^{8}$. Approximating absorbed dose by collision kerma is commonly employed by most MC codes used for brachytherapy dosimetry.

While estimating relative $2 \mathrm{D}$ dose distributions in medium around ${ }^{125} \mathrm{I}$ sources from measurements and MC methods ${ }^{9}$ was relatively common by the mid-1980's, the 
dose rate constant (dose at a reference point in medium per unit source strength) for low energy seeds had not been definitively measured or calculated until the late 1980s. Williamson performed simulations in 1988 for models 6711, 6702 and $67013 \mathrm{M}$ sources as well as the National Institute of Standards and Technology (NIST) Ritz low energy free air chamber, used as air kerma strength standard, to obtain dose rate constants $^{10}$. By including the effects of $4.5 \mathrm{keV} \mathrm{Ti} K$ x-rays produced in the source encapsulation, first experimentally observed by $\mathrm{Kubo}^{11}$, Williamson showed that ${ }^{125}$, absolute dose rates obtained from semi-empirical methods were overestimated by $10 \%-14 \%$.

The 1990s saw several studies comparing MC simulations of brachytherapy sources and thermoluminescent dosimetry (TLD) measurements ${ }^{12,13}$. Williamson observed good agreement (1\%-5\%) between simulations and measurements for ${ }^{125}$ I when accounting for the measurement phantom medium in the simulations ${ }^{14}$. Excellent agreement was observed for ${ }^{192} \operatorname{Ir}(2 \%-3 \%)$, when the shape and size of the measurement phantom were modeled although the influence of its composition was found to be less important in this energy range ${ }^{14}$. In a significant series of articles, Williamson's group performed extensive benchmarking of MC photon-transport calculations against precision diode measurements in water showing that $\mathrm{MC}$ accurately (1\%-3\%) reproduced both relative and absolute dose rates across the entire brachytherapy energy range in both homogeneous and heterogeneous phantom geometries ${ }^{14-19}$. These results confirmed that the MC methodology applied to brachytherapy dosimetry was mature and sufficiently accurate and robust to support clinical dosimetry. This was reinforced by the American Association of Physicists in Medicine (AAPM) Task Group No. 43 (TG-43) requirement that at least one experimental and one MC determination of dosimetry parameters be published before using a source clinically, essentially making MC simulations an industry standard ${ }^{20}$. However, $M C$ results should not be trusted blindly, as illustrated by the significant dose-estimation errors resulting from use of obsolete low energy cross sections data ${ }^{21-23}$, a problem first pointed out by Williamson $^{14}$. For brachytherapy dose distributions in the $<50 \mathrm{keV}$ energy range, where energy deposition is dominated by photo-electric absorption, even $1 \%-2 \%$ errors in the photoelectric cross section can give rise to dose computation errors as large as $10 \%-15 \%$ at $5 \mathrm{~cm}$ distance. This led to the adoption of modern cross section libraries derived largely from theoretical quantum mechanical models ${ }^{24}$. The reader is referred to Williamson and Rivard for a more detailed discussion of this complex issue ${ }^{25}$.

The rising popularity of prostate seed implantation in the United States, increasing from 5,000 procedures in 1995 to about 50,000 in 2002, fuelled a rise in the number of commercially available brachytherapy seeds and designs ${ }^{26}$. While the initial 1995 TG43 report presented consensus dosimetry parameters for two ${ }^{125} \mathrm{I}$ and one ${ }^{103} \mathrm{Pd}$ seeds ${ }^{27}$, its 2004 low-energy seed update (TG-43U1) presented data for 8 seed models ${ }^{28}$, while the 2007 supplement presented data from an additional 8 seed mod$\mathrm{els}^{29}$. Yet another supplement is in preparation for the remaining commercially available low-energy photon emitting sources. The recently published joint AAPM/ESTRO report $^{30}$ on high energy brachytherapy dosimetry applied the AAPM prerequisite ${ }^{31}$ to $21{ }^{192} \mathrm{Ir},{ }^{137} \mathrm{Cs}$, and ${ }^{60}$ Co source models. This increasing proliferation of new brachy- 
therapy sources and dosimetry datasets was associated with a rapidly growing number of $\mathrm{MC}$ related brachytherapy publications in the peer-reviewed literature (Figure 2.1).

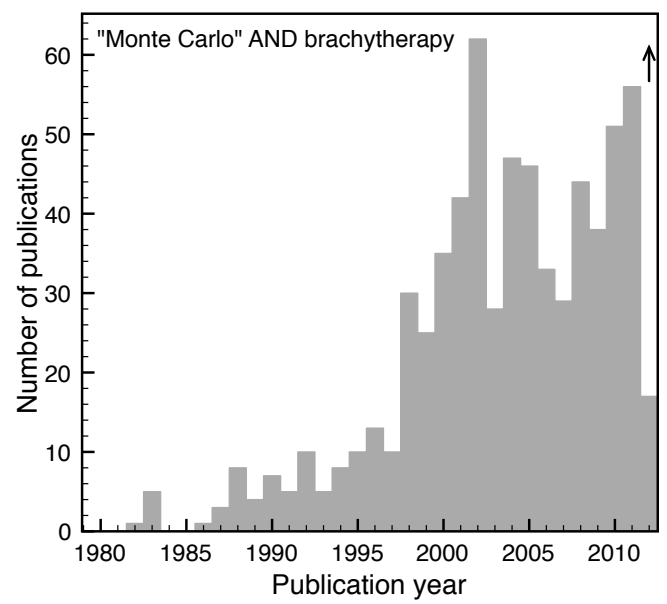

Figure 2.1: Results of a PubMed search (11 April 2012) for the terms MC and brachytherapy presented per publication year. The arrow indicates the extrapolated 2012 number of publications to a complete year $(=61)$.

The use of MC methods in brachytherapy now goes beyond single-source dosimetry. An important area of current research is application of MC simulations to perform patient specific dose calculations. This effort is necessary to overcome the limitations of TG-43-style table-based source-superposition algorithms, which include neglecting interseed attenuation and tissue heterogeneities for low-energy seed implants ${ }^{32-34}$ and neglect of applicator shielding and partial scattering effects for higher energy brachytherapy procedures ${ }^{13,35-37}$. Several MC dose calculation platforms, generally based on CT images, have been presented in the literature ${ }^{38-41}$. For low-energy sources, the biggest challenge to accurate patient-specific dosimetry is accurate voxel-by-voxel assignment of photon cross sections tables.

\section{THE TREATMENT OF INHOMOGENEITIES}

The TG-43 formalism, based on the work of the Interstitial Collaborative Working Group (ICWG) ${ }^{42}$, was a major step forward in brachytherapy dose calculation. It replaced the use of semi empirical calculation methods based on apparent activity, equivalent mass of radium, exposure-rate constants, and tissue-attenuation coefficients, quantities that are not source-model but only radionuclide dependent. By employing dosimetry parameters that are dependent on the radionuclide, its distribution in the source, and the source geometry itself, and by recommending measured or 
calculated consensus datasets, the formalism of the TG-43 protocol, and its subsequent updates, improved standardization and accuracy in brachytherapy dose calculations. Fifteen years later, the widely-cited (over 900 citations as of April 2012 [Web of Science 17 April 2012, Times cited=915]) and -adopted formalism is still heavily used, ensuring consistency, standardization and comparability of brachytherapy dose calculations across institutions worldwide.

Since dose calculations based on the TG-43 formalism rely on the superposition of single source dose distributions over the dwell or seed positions used for treatment, dose distributions can be obtained with minimal calculation time. This fast and practical method facilitates clinical practices such as transrectal ultrasound image-guided live planning and dose distribution optimization. However, the inherent simplicity of the TG-43 formalism can lead to inaccurate dose distributions when the calculation geometry deviates significantly from the reference water sphere used to derive the source's dosimetric parameters. Patient tissue compositions, densities, and dimensions deviate significantly from the reference geometry, which can result in large discrepancies between estimated and delivered dose, especially for low energy sources. Generally, TG-43 dose calculation limitations can be attributed to five phenomena: absorption, attenuation, shielding, scattering, and breakdown of the kerma approximation for absorbed dose. Depending on source energy and anatomic site, some or all of these phenomena may induce significant dose calculation errors. Table 2.1 lists, for both high- and low-energy brachytherapy sources, sites where the formalism leads to significant dose calculation errors ${ }^{43}$. The following sections briefly discuss these aspects.

Table 2.1: List of anatomic sites where the limitations of the TG-43 dose calculation formalism lead to significant dose calculation errors (indicated by " $Y$ "). From Rivard et al. ${ }^{43}$

\begin{tabular}{|c|c|c|c|c|c|}
\hline $\begin{array}{l}\text { Anatomic } \\
\text { site }\end{array}$ & $\begin{array}{l}\text { Source } \\
\text { energy }\end{array}$ & Absorption & Attenuation & Shielding & Scattering \\
\hline \multirow[t]{2}{*}{ Prostate } & High & $\mathrm{N}$ & $\mathrm{N}$ & $\mathrm{N}$ & $\mathrm{N}$ \\
\hline & Low & $Y$ & $Y$ & $Y$ & $\mathrm{~N}$ \\
\hline \multirow[t]{2}{*}{ Breast } & High & $\mathrm{N}$ & $\mathrm{N}$ & $\mathrm{N}$ & $Y$ \\
\hline & Low & $Y$ & $Y$ & $Y$ & $\mathrm{~N}$ \\
\hline \multirow[t]{2}{*}{ GYN } & High & $\mathrm{N}$ & $\mathrm{N}$ & $Y$ & $\mathrm{~N}$ \\
\hline & Low & $Y$ & $Y$ & $\mathrm{~N}$ & $\mathrm{~N}$ \\
\hline \multirow[t]{2}{*}{ Skin } & High & $\mathrm{N}$ & $\mathrm{N}$ & Y & $Y$ \\
\hline & Low & $Y$ & $\mathrm{~N}$ & $Y$ & $\mathrm{Y}$ \\
\hline \multirow[t]{2}{*}{ Lung } & High & $\mathrm{N}$ & $\mathrm{N}$ & $\mathrm{N}$ & $Y$ \\
\hline & Low & $Y$ & $Y$ & $\mathrm{~N}$ & $Y$ \\
\hline \multirow[t]{2}{*}{ Penis } & High & $\mathrm{N}$ & $\mathrm{N}$ & $\mathrm{N}$ & $Y$ \\
\hline & Low & $Y$ & $\mathrm{~N}$ & $\mathrm{~N}$ & $Y$ \\
\hline \multirow[t]{2}{*}{ Eye } & High & $\mathrm{N}$ & $\mathrm{N}$ & $Y$ & $Y$ \\
\hline & Low & $Y$ & $Y$ & $Y$ & $Y$ \\
\hline
\end{tabular}




\section{Absorbed dose differences between water and human tissues}

At low photon energies, the mass-energy absorption coefficient $\mu_{\text {en }} / \rho$ varies significantly between tissues due to the importance of the approximately $Z^{3}$ dependence of the photoelectric cross section. For a given photon energy fluence under conditions of charged particle equilibrium, the absorbed dose-to-water and -tissue are related by $D_{\text {tissue }} /\left(\mu_{\mathrm{en}} / \rho\right)_{\text {tissue }}=D_{\text {water }} /\left(\mu_{\mathrm{en}} / \rho\right)_{\text {water }}$. As seen in Figure 2.2 , the ratio $\left(\mu_{\mathrm{en}} / \rho\right)_{\text {tissue }} /\left(\mu_{\mathrm{en}} / \rho\right)_{\text {water }}$ differs from unity for most tissues in the energy range of lowenergy sources. At high photon energies, the ratios converge to unity as the Compton cross section becomes more important. This gives rise to significant dependence of the fluence-to-kerma conversion factor on tissue elemental composition and mass density ${ }^{44}$.

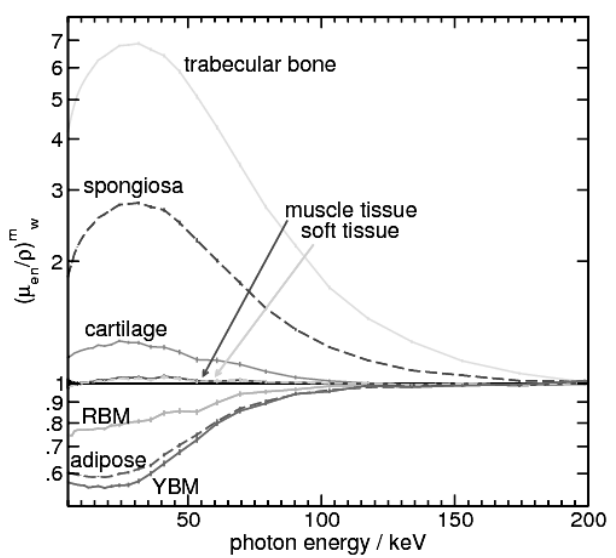

Figure 2.2: The EGSnrc ${ }^{45}$ user code ' $g$ ' was used to calculate $\mu_{e n} / \rho$ for a series of human tissues taken from ICRU Report $46^{46}$ and ICRP Report $89^{47}$. RBM and YBM stand for red and yellow bone marrow from "active and inactive marrow" in ICRP Report $89^{47}$. From Beaulieu et al. ${ }^{48}$

\section{Attenuation differences between water and human tissues}

As the linear attenuation coefficient $\mu$ is proportional to $\rho$, density differences between water and human tissues will result in different photon energy fluence and dose distributions, for both low- and high-energy sources. Mass attenuation coefficients $\mu / \rho$ also vary between tissues and water at low photon energies because of the aforementioned dependence of photoelectric cross section on atomic number. As $\mu / \rho$ is larger at low photon energies (disregarding the absorption edges), differences of elemental composition or density will result in larger dose differences for low-energy sources. 


\section{Shielding}

In multisource implants, photons emitted from a given source can be absorbed by the radio-opaque markers (e.g., $\mathrm{Au}, \mathrm{Ag}, \mathrm{Pb}$ ) or the radio-opaque components of adjacent sources, causing a lower dose than predicted by TG-43. This is generally referred to as interseed attenuation (ISA) in the literature. For HDR high-energy sources, ISA is not an issue since a single source steps through the implanted applicators, treating one dwell position at a time. However, applicator materials such as stainless steel can cause deviations. Some applicators also contain high atomic number, high density shielding material such as tungsten, used to protect organs at risk. The most common are shielded vaginal and intrarectal applicators

\section{Scattering conditions}

TG-43 parameters are calculated in water spheres with radii of $15 \mathrm{~cm}$ and $40 \mathrm{~cm}$ for low- and high-energy sources, respectively, ensuring TG-43 calculations represent doses in unbounded water medium over the therapeutically relevant distance range. Situations where full scatter conditions are not met, sources close to the skin for example, will result in deviation of delivered dose from TG-43. Given the longer pathlength and higher Compton scattering cross section, high-energy photons are more sensitive to geometries with tissue boundaries near the implanted sources. Example clinical geometries include the breast, skin, and lung ${ }^{36}$.

\section{Magnitude of clinical impact}

Several studies have compared MC dose calculations to the results of the TG-43 formalism. Meigooni et al. were the first to investigate interseed attenuation (ISA) in 1992 using TLD measurements and estimated that dose reductions of $6 \%$ could be expected at the edge of $\mathrm{a}^{125} \mathrm{I}$ prostate implant ${ }^{49}$. Chibani et al. and Carrier et al. published results of $\mathrm{MC}$ simulations performed in real ${ }^{125} \mathrm{I}$ and ${ }^{103} \mathrm{Pd}$ prostate implant geometries in 2005 and 2006 respectively, finding $D_{90}$ reductions of $2 \%-5 \%$ due to ISA ${ }^{50}$, 51 . Chibani et al. also investigated the dosimetric impact of the presence of calcifications in the prostate and found $D_{90}$ reductions of up to $37 \%$. Carrier et al. subsequently performed a retrospective study of 28 prostate cancer patients implanted with ${ }^{125}$ I using post-implant CT data (Figure 2.3) based upon ICRP recommended tissue compositions ${ }^{52}$. They found an average $D_{90}$ decrease of $7 \%$ due to ISA and tissue composition $^{53}$. Afsharpour et al. performed a similar study for breast brachytherapy with ${ }^{103} \mathrm{Pd}$ implants ${ }^{54}$. They found $D_{90}$ reductions ranging from $4 \%$ for an all-gland breast to $35 \%$ for an all-adipose breast. This increased sensitivity of breast brachytherapy compared to prostate implants is caused in part by the lower energy of ${ }^{103} \mathrm{Pd}$, but mostly by the larger deviation of adipose tissue's effective atomic number to that of water compared to the relatively small difference between ICRP prostate and water. All studies listed above reported dose to tissue. 


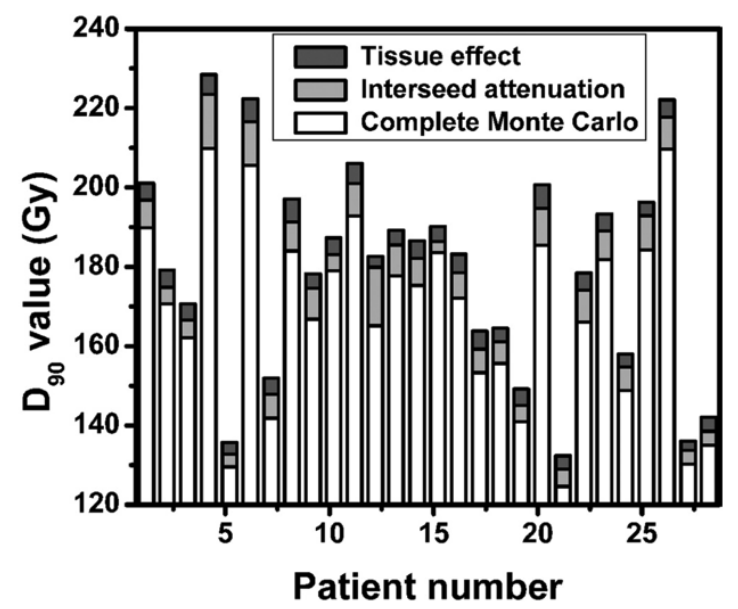

Figure 2.3: Impact of interseed attenuation and tissue nonwater equivalence on the clinical parameter $D_{90}$ from post implant dosimetry of 28 prostate cancer patients treated with ${ }^{125} \mathrm{I}$ implants. Tissue effect and Interseed attenuation represent the $D_{90}$ reductions from the TG-43 estimate from each effect. Complete Monte Carlo represents the $D_{90}$ parameter when accounting for both effects. From Carrier et $a .^{53}$

Melhus and Rivard showed that tissue effects were negligible for ${ }^{192}$ Ir over clinically relevant distances in soft tissue, muscle, and breast using point sources in uniform spherical phantoms ${ }^{55}$. This is due to the higher photon energies of ${ }^{192} \mathrm{Ir}$. On the other hand, applicator shielding, designed to spare organs at risk, can cause dose reductions of $5 \%-11 \%$ for GYN applicators ${ }^{56}$ and up to $24 \%$ for endorectal applicators when compared to the TG-43 predicted dose ${ }^{57}$. Poon et al. showed that TG-43 dose distributions for ${ }^{192}$ Ir breast implants overestimated dose to the skin by $5 \%$, due to differences from full scatter conditions with $\mathrm{MC}$ simulations ${ }^{36}$, confirming earlier findings from Pantelis et $a l^{58}$.

\section{MC dose calculation tools}

\section{ALGEBRA}

ALGEBRA (ALgorithm for heterogeneous dosimetry based on Geant4 for BRAchytherapy) is based on the general purpose MC code Geant4 and the DICOM RT standard. Initially developed by Carrier and co-workers ${ }^{53}$, and referred to as Geant4/DICOMRT in the recent review by Rivard et al. $^{59}$ the code was later modified by Afsharpour and given its current name ${ }^{39}$. The code can import planning data from a TPS (seed 
position, air kerma strength, etc.) and structural data (contours). CT images are imported and a semi automatic segmentation method uses the CT calibration curve to assign densities and organ contours to assign elemental compositions to each voxel. Seed geometries and voxels do not overlap in ALGEBRA; voxels intersecting seeds are discarded, and the missing volume is replaced by water. The new layered mass geometry (LMG) functionality of Geant4, where the overlap of seeds and voxels is permitted, should eliminate the need to perform this step $^{60}$. Dose scoring is performed using the 'parallel world' functionality of Geant4 by creating a scoring mesh independent of the transport geometry. The resolution of the CT derived transport geometry can be modified, as well as that of the scoring mesh, and they need not be equivalent. Secondary electrons are immediately discarded and collision kerma is scored with a pathlength estimator. Both kerma in water or medium by transporting photons in medium, $K_{\mathrm{w}, \mathrm{m}}$ and $K_{\mathrm{m}, \mathrm{m}}$, can be scored. The use of source phase space files is also possible. Calculation times in ALGEBRA for $2 \times 2 \times 2 \mathrm{~mm}^{3}$ voxels are about 6 and 12 minutes for $2 \%$ statistical uncertainty for a breast ${ }^{103} \mathrm{Pd}$ implant and a prostate ${ }^{125} \mathrm{I}$ implant, respectively.

\section{Other tools}

MCPI (Monte Carlo dose calculation for prostate implant) was developed by Chibani and co-workers in 2005 to perform patient specific prostate implant dose calculations with ${ }^{125} \mathrm{I}$ and ${ }^{103} \mathrm{Pd}{ }^{41}$. The code is based on the general purpose MC software GEPTS ${ }^{61}$, using only photon transport for low-energy ( $<1 \mathrm{MeV}$ ) sources. Several variance reduction techniques, in addition to the track-length collision kerma estimator, were developed for $\mathrm{MCPI}$.

The correlated sampling technique ${ }^{62}$ has recently been implemented in Williamson's PTRAN_CT ${ }^{63}$ by Sampson and co-workers ${ }^{38}$. The PTRAN_CT implementation also included the variance reduction techniques of $\mathrm{MCPI}$ described above and expectedvalue track-length scoring. This code can input DICOM CT images and currently uses the EGSnrc CTcreate software to assign cross-section files based upon single-energy CT. An alternative could be the user interface BrachyGUI, developed at McGill University ${ }^{64}$.

BRACHYDOSE is an EGSnrc ${ }^{45}$ user code utilizing Yegin's multi-geometry package ${ }^{65}$ and was developed to perform prostate implant dose calculations ${ }^{66}$. The code has been extensively benchmarked by generating single source TG-43 parameters for several commercially available ${ }^{125} \mathrm{I},{ }^{103} \mathrm{Pd}$ and ${ }^{192}$ Ir sources ${ }^{40}$.

\section{Issues involved in clinical implementation}

$D_{w, m}$ or $D_{m, m}$

An excellent overview of this issue can be found in the report of AAPM Task Group $186^{48}$. MC simulations provide the ability to calculate the energy deposition in the medium present in each voxel of a treatment geometry, either from photons $\left(K_{\mathrm{m}, \mathrm{m}}\right)$ 
or electrons set in motion by photons $\left(D_{\mathrm{m}, \mathrm{m}}\right)$. An alternative approach to reporting dose to medium would be to calculate the energy deposition in a unique reference medium. For historical/practical reasons, this reference medium has consistently been water. While in EBRT $D_{\mathrm{w}, \mathrm{m}} / D_{\mathrm{m}, \mathrm{m}}$ differences between soft tissues and water are of the order of $2 \%$, at the energies of brachytherapy sources these differences can be much larger. In EBRT, conversion is performed by using ratios of mass collision stopping power under the assumptions of Bragg-Gray cavity theory (electron ranges larger than size of cavity). For brachytherapy, the method of conversion depends on the cavity size. Millimeter sized voxels are several times larger than the range of secondary electrons produced by photons emitted from low- or high-energy sources, thus ratios of mass energy absorption coefficients $\mu_{\mathrm{en}} / \rho$ (large cavity theory) may be used to convert $K_{\mathrm{m}, \mathrm{m}}$ to $K_{\mathrm{w}, \mathrm{m}}$. The $\mu_{\mathrm{en}} / \rho$ differences between tissue and water are generally important for low-energy sources (up to $80 \%$ for certain soft tissues), while the situation is similar to EBRT for high-energy brachytherapy sources (3\% - 5\%).

Complications arise when we consider that the radiation response of tissues and tumors correlates with the energy deposited in the nuclei of cells. The dimensions of mammalian cells $(\sim 10 \mu \mathrm{m})$ mean that they act as Bragg Gray or small cavities at the energies of high-energy brachytherapy sources and as intermediate cavities bracketed by large and small cavity theories at the energies of low-energy brachytherapy sources. This makes the conversion of $D_{\mathrm{m}, \mathrm{m}}$ to $D_{\mathrm{w}, \mathrm{m}}$ using $\mu_{\mathrm{en}}$ ratios questionable for low-energy sources and means that different conversion methods are required across the brachytherapy energy range. There is also no clear indication that water is the best material to represent the nuclei of various cells ${ }^{67}$. For these reasons, it has been recommended to report both $D_{\mathrm{m}, \mathrm{m}}$ and $D_{\mathrm{w}, \mathrm{m}}$ when performing $\mathrm{MC}$ dose calculations for brachythera$\mathrm{py}^{48}$.

\section{Tissue composition selection}

MC dose calculations generally require voxel-by-voxel assignment of tissue density and elemental composition (mass fraction of each element composing the tissue). While tissue composition essentially plays no role for brachytherapy MC dose calculations with high-energy sources, it becomes very important for low-energy sources due to the $Z$ dependence of $\mu / \rho$ and $\mu_{\mathrm{en}} / \rho$ (photoelectric effect). While tissue density also affects dose distributions, it is readily obtained from CT images of the treated anatomy using a Hounsfield Unit (HU) to density calibration (similar to the HU to electron density calibration used in external beam).

Soft tissue composition, on the other hand, is difficult to correlate with $\mathrm{HU}$, as several human tissues possessing different compositions have $\mathrm{HU}$ falling in the [$100,100]$ range around water ${ }^{68}$. In most MC brachytherapy studies, tissue composition is assigned on the basis of anatomical organ contours, i.e. prostate tissue is uniformly assigned to the prostate contour and breast tissue is uniformly assigned to the breast contour. With this practice, the question of what constitutes prostate or breast tissue arises. Literature reports ${ }^{46,52}$ provide compositions for a wide range of human tissues, 
however these compositions are based on old (1930-1970) measurements on a small number of subjects and may be of questionable accuracy in characterizing average tissue composition in the population served by brachytherapy. Some reports provide a range of composition for a given tissue, an indication of inter-sample variability ${ }^{69}$. Only one modern study of elemental composition of tumors appears to exist, that of Maughan et al. $^{70}$, which demonstrated a large variation in carbon content $(8 \%$ to $32 \%$ by weight) and mineral ash ( $0.9 \%$ to $3 \%$ by weight) which gives to variation in $\left(\mu_{\text {en }} / \rho\right)_{\text {water }}^{\text {tumor }}$ of $20 \%$ at $30 \mathrm{keV}$.

\section{CT image usage}

CT images are generally the standard input to MC calculation, as CT image intensity approximately tracks the relative linear attenuation coefficient, evaluated at the effective scanning energy, of the underlying tissue. Converting $\mathrm{HU}$ to electron or mass density is a relatively straightforward step, as explained above. For low energy sources, the issues of the previous section demand special attention, but for high-energy sources, where tissue composition effects are essentially negligible, CT images provide all the necessary information to accurately calculate the dose. Additionally, the location of implanted seeds, catheters, or applicators are easily derived from CT images.

CT artifacts, especially those caused by the high density components of implanted LDR seeds (streaking artifacts) and other foreign metal bodies implanted in the body, can seriously degrade the information required for accurate dose calculations. Metal artifact correction algorithms exist and can alleviate this problem ${ }^{71}$. However, metal artifact reduction cannot be considered to be a solved problem, especially at the level of quantitative accuracy needed to specify tissue composition for low-energy brachytherapy. Even relatively subtle streaks can degrade voxel-by-voxel single energy CT tissue identity analyses ${ }^{72}$.

\section{REFERENCES}

${ }^{1}$ J.F. Williamson, "The Sievert integral revisited: evaluation and extension to ${ }^{125} \mathrm{I},{ }^{169} \mathrm{Yb}$, and ${ }^{192} \mathrm{Ir}$ brachytherapy sources," Int J Radiat Oncol Biol Phys 36, 1239-1250 (1996).

2J.F. Williamson and M.J. Rivard, Quantitative dosimetry methods for brachytherapy, in Brachytherapy Physics: Second Edition, B.R. Thomadsen, M.J. Rivard, and W.M. Butler, Editors. 2005, Medical Physics Publishing: Madison, WI. p. 233-294.

${ }^{3}$ L.L. Meisberger, R.J. Keller, and R.J. Shalek, "The effective attenuation in water of the gamma rays of gold 198, iridium 192, cesium 137 , radium 226, and cobalt 60 ," Radiology 90 , 953957 (1968).

${ }^{4}$ R.G. Dale, "Some theoretical derivations relating to the tissue dosimetry of brachytherapy nuclides, with particular reference to iodine-125," Med Phys 10, 176-183 (1983).

${ }^{5} \mathrm{~V}$. Krishnaswamy, "Calculation of the dose distribution about californium-252 needles in tissue," Radiology 98, 155-160 (1971). 
${ }^{6}$ J.F. Williamson, R.L. Morin, and F.M. Khan, "Monte Carlo evaluation of the Sievert integral for brachytherapy dosimetry," Phys Med Biol 28, 1021-1032 (1983).

${ }^{7}$ G.S. Burns and D.E. Raeside, "Two-dimensional dose distribution around a commercial ${ }^{125}$, seed," Med Phys 15, 56-60 (1988).

${ }^{8}$ J.F. Williamson, "Monte Carlo evaluation of kerma at a point for photon transport problems," Med Phys 14, 567-576 (1987).

${ }^{9}$ J.F. Williamson and F.J. Quintero, "Theoretical evaluation of dose distributions in water about models 6711 and $6702^{125}$ I seeds," Med Phys 15, 891-897 (1988).

${ }^{10}$ J.F. Williamson, "Monte Carlo evaluation of specific dose constants in water for ${ }^{125}$ I seeds," Med Phys 15, 686-694 (1988).

${ }^{11} \mathrm{H}$. Kubo, "Exposure contribution from Ti $\mathrm{K} x$ rays produced in the titanium capsule of the clinical I-125 seed," Med Phys 12, 215-220 (1985).

${ }^{12}$ A. Kirov, J.F. Williamson, A.S. Meigooni, and Y. Zhu, "TLD, diode and Monte Carlo dosimetry of an ${ }^{192}$ Ir source for high dose-rate brachytherapy," Phys Med Biol 40, 2015-2036 (1995).

${ }^{13}$ R.K. Valicenti, A.S. Kirov, A.S. Meigooni, V. Mishra, R.K. Das, and J.F. Williamson, "Experimental validation of Monte Carlo dose calculations about a high-intensity Ir-192 source for pulsed dose-rate brachytherapy," Med Phys 22, 821-829 (1995).

${ }^{14}$ J.F. Williamson, "Comparison of measured and calculated dose rates in water near I-125 and Ir192 seeds," Med Phys 18, 776-786 (1991).

${ }^{15}$ J.F. Williamson, H. Perera, Z. Li, and W.R. Lutz, "Comparison of calculated and measured heterogeneity correction factors for ${ }^{125} \mathrm{I},{ }^{137} \mathrm{Cs}$, and ${ }^{192} \mathrm{Ir}$ brachytherapy sources near localized heterogeneities," Med Phys 20, 209-222 (1993).

${ }^{16}$ H. Perera, J.F. Williamson, Z. Li, V. Mishra, and A.S. Meigooni, "Dosimetric characteristics, airkerma strength calibration and verification of Monte Carlo simulation for a new Ytterbium169 brachytherapy source," Int J Radiat Oncol Biol Phys 28, 953-970 (1994).

${ }^{17}$ R.K. Das, Z. Li, H. Perera, and J.F. Williamson, "Accuracy of Monte Carlo photon transport simulation in characterizing brachytherapy dosimeter energy-response artefacts," Phys Med Biol 41, 995-1006 (1996).

${ }^{18}$ R. Das, A.S. Meigooni, V. Mishra, M.A. Langton, and J.F. Williamson, "Dosimetric characteristics of the type 8 Ytterbium-169 interstitial brachytherapy source," Journal of Brachytherapy International 13, 219-234 (1997).

${ }^{19}$ R.K. Das, D. Keleti, Y. Zhu, A.S. Kirov, A.S. Meigooni, and J.F. Williamson, "Validation of Monte Carlo dose calculations near ${ }^{125}$ I sources in the presence of bounded heterogeneities," Int J Radiat Oncol Biol Phys 38, 843-853 (1997).

${ }^{20}$ M.J. Rivard, et al., "Update of AAPM Task Group No. 43 Report: A revised AAPM protocol for brachytherapy dose calculations," Med Phys 31, 633-674 (2004).

${ }^{21}$ T.D. Bohm, P.M. DeLuca, Jr., and L.A. DeWerd, "Brachytherapy dosimetry of ${ }^{125} \mathrm{I}$ and ${ }^{103} \mathrm{Pd}$ sources using an updated cross section library for the MCNP Monte Carlo transport code," Med Phys 30, 701-711 (2003).

${ }^{22}$ B. Reniers, F. Verhaegen, and S. Vynckier, "The radial dose function of low-energy brachytherapy seeds in different solid phantoms: comparison between calculations with the EGSnrc and MCNP4C Monte Carlo codes and measurements," Phys Med Biol 49, 15691582 (2004).

${ }^{23}$ J.J. Demarco, R.E. Wallace, and K. Boedeker, "An analysis of MCNP cross-sections and tally methods for low-energy photon emitters," Phys Med Biol 47, 1321-1332 (2002).

${ }^{24}$ D. Cullen, J.H. Hubbell, and L. Kissel, "EPDL97: the Evaluated Photon Data Library, 97 version," UCRL-50400 Vol. 6, Rev 5, (1997).

${ }^{25}$ J.F. Williamson and M.J. Rivard, Thermoluminescent Detector and Monte Carlo techniques for Reference-Quality Brachytherapy Dosimetry, in Clinical Dosimetry Measurements in 
Radiotherapy (AAPM 2009 Summer School), D.W.O. Rogers and J. Cygler, Editors. 2009, Medical Physics Publishing: Madison, WI. p. 437-499.

${ }^{26}$ P. Grimm and J. Sylvester, "Advances in brachytherapy," Rev Urol 6 Suppl 4, S37-48 (2004).

${ }^{27}$ R. Nath, L.L. Anderson, G. Luxton, K.A. Weaver, J.F. Williamson, and A.S. Meigooni, "Dosimetry of interstitial brachytherapy sources: recommendations of the AAPM Radiation Therapy Committee Task Group No. 43. American Association of Physicists in Medicine," Med Phys 22, 209-234 (1995).

${ }^{28}$ M.J. Rivard, et al., "Update of AAPM Task Group No. 43 Report: A revised AAPM protocol for brachytherapy dose calculations," Med Phys 31, 633-674 (2004).

${ }^{29}$ M.J. Rivard, et al., "Supplement to the 2004 update of the AAPM Task Group No. 43 Report," Med Phys 34, 2187-2205 (2007).

${ }^{30} \mathrm{~J}$. Perez-Calatayud, et al., "Dose calculation for photon-emitting brachytherapy sources with average energy higher than $50 \mathrm{keV}$ : Report of the AAPM and ESTRO," Med Phys 39, 29042929 (2012).

${ }^{31} \mathrm{Z}$. Li, et al., "Dosimetric prerequisites for routine clinical use of photon emitting brachytherapy sources with average energy higher than 50 kev," Med Phys 34, 37-40 (2007).

${ }^{32}$ G.S. Burns and D.E. Raeside, "The accuracy of single-seed dose superposition for I-125 implants," Med Phys 16, 627-631 (1989).

${ }^{33}$ J.F. Carrier, M. D'Amours, F. Verhaegen, B. Reniers, A.G. Martin, E. Vigneault, and L. Beaulieu, "Postimplant dosimetry using a Monte Carlo dose calculation engine: a new clinical standard," Int J Radiat Oncol Biol Phys 68, 1190-1198 (2007).

${ }^{34}$ O. Chibani, J.F. Williamson, and D. Todor, "Dosimetric effects of seed anisotropy and interseed attenuation for 103Pd and 125I prostate implants," Med Phys 32, 2557-2566 (2005).

${ }^{35}$ E. Poon, B. Reniers, S. Devic, T. Vuong, and F. Verhaegen, "Dosimetric characterization of a novel intracavitary mold applicator for ${ }^{192} \mathrm{Ir}$ high dose rate endorectal brachytherapy treatment," Med Phys 33, 4515-4526 (2006).

${ }^{36} \mathrm{E}$. Poon and F. Verhaegen, "Development of a scatter correction technique and its application to HDR ${ }^{192}$ Ir multicatheter breast brachytherapy," Med Phys 36, 3703-3713 (2009).

${ }^{37}$ K.A. Gifford, J.L. Horton, Jr., C.E. Pelloski, A. Jhingran, L.E. Court, F. Mourtada, and P.J. Eifel, "A three-dimensional computed tomography-assisted Monte Carlo evaluation of ovoid shielding on the dose to the bladder and rectum in intracavitary radiotherapy for cervical cancer," Int J Radiat Oncol Biol Phys 63, 615-621 (2005).

${ }^{38}$ A. Sampson, Y. Le, and J.F. Williamson, "Fast patient-specific Monte Carlo brachytherapy dose calculations via the correlated sampling variance reduction technique," Med Phys 39, 10581068 (2012).

${ }^{39} \mathrm{H}$. Afsharpour, et al., "ALGEBRA: ALgorithm for the heterogeneous dosimetry based on GEANT4 for BRAchytherapy," Phys Med Biol 57, 3273-3280 (2012).

${ }^{40}$ R.E. Taylor, G. Yegin, and D.W. Rogers, "Benchmarking brachydose: Voxel based EGSnrc Monte Carlo calculations of TG-43 dosimetry parameters," Med Phys 34, 445-457 (2007).

${ }^{41}$ O. Chibani and J.F. Williamson, "MCPI: a sub-minute Monte Carlo dose calculation engine for prostate implants," Med Phys 32, 3688-3698 (2005).

${ }^{42}$ L.L. Anderson, R. Nath, and K.A. Weaver, Interstitial Collaborative Working Group (ICWG). Interstitial Brachytherapy: Physical, Biological, and Clinical Considerations. 1990, New York: Raven.

${ }^{43}$ M.J. Rivard, J.L. Venselaar, and L. Beaulieu, "The evolution of brachytherapy treatment planning," Med Phys 36, 2136-2153 (2009).

${ }^{44}$ G. Landry, B. Reniers, J.P. Pignol, L. Beaulieu, and F. Verhaegen, "The difference of scoring dose to water or tissues in Monte Carlo dose calculations for low energy brachytherapy photon sources," Med Phys 38, 1526-1533 (2011). 
${ }^{45}$ I. Kawrakow, "Accurate condensed history Monte Carlo simulation of electron transport. I. EGSnrc, the new EGS4 version," Med Phys 27, 485-498 (2000).

${ }^{46}$ ICRU, Report 46: Photon, electron, proton and neutron interaction data for body tissues. 1992, Bethesda, MD.

${ }^{47}$ J. Valentin, "Basic anatomical and physiological data for use in radiological protection: reference values: ICRP Publication 89," Annals of the ICRP 32, 1-277 (2002).

${ }^{48}$ L. Beaulieu, et al., "Report TG-186 of the AAPM, ESTRO, and ABG on model-based dose calculation techniques in brachytherapy: Status and clinical requirements for implementation beyond the TG-43 formalism," Med Phys XX, xx-yy (2012).

${ }^{49}$ A.S. Meigooni, J.A. Meli, and R. Nath, "Interseed effects on dose for ${ }^{125}$ I brachytherapy implants," Med Phys 19, 385-390 (1992).

${ }^{50}$ O. Chibani, J.F. Williamson, and D. Todor, "Dosimetric effects of seed anisotropy and interseed attenuation for ${ }^{103} \mathrm{Pd}$ and ${ }^{125}$ I prostate implants," Med Phys 32, 2557-2566 (2005).

${ }^{51}$ J.F. Carrier, L. Beaulieu, F. Therriault-Proulx, and R. Roy, "Impact of interseed attenuation and tissue composition for permanent prostate implants," Med Phys 33, 595-604 (2006).

${ }^{52}$ ICRP, Report of the task group on reference man, ICRP Report 23. 1975, Washington D.C.

${ }^{53}$ J.F. Carrier, M. D'Amours, F. Verhaegen, B. Reniers, A.G. Martin, E. Vigneault, and L. Beaulieu, "Postimplant dosimetry using a Monte Carlo dose calculation engine: a new clinical standard," Int J Radiat Oncol Biol Phys 68, 1190-1198 (2007).

${ }^{54}$ H. Afsharpour, J.P. Pignol, B. Keller, J.F. Carrier, B. Reniers, F. Verhaegen, and L. Beaulieu, "Influence of breast composition and interseed attenuation in dose calculations for postimplant assessment of permanent breast ${ }^{103}$ Pd seed implant," Phys Med Biol 55, 4547-4561 (2010).

${ }^{55}$ C.S. Melhus and M.J. Rivard, "Approaches to calculating AAPM TG-43 brachytherapy dosimetry parameters for ${ }^{137} \mathrm{Cs},{ }^{125} \mathrm{I},{ }^{192} \mathrm{Ir},{ }^{103} \mathrm{Pd}$, and ${ }^{169} \mathrm{Yb}$ sources," Med Phys 33, 1729-1737 (2006).

${ }^{56}$ J. Markman, J.F. Williamson, J.F. Dempsey, and D.A. Low, "On the validity of the superposition principle in dose calculations for intracavitary implants with shielded vaginal colpostats," Med Phys 28, 147-155 (2001).

${ }^{57}$ E. Poon, J.F. Williamson, T. Vuong, and F. Verhaegen, "Patient-specific Monte Carlo dose calculations for high-dose-rate endorectal brachytherapy with shielded intracavitary applicator," Int J Radiat Oncol Biol Phys 72, 1259-1266 (2008).

${ }^{58} \mathrm{E}$. Pantelis, et al., "The effect of finite patient dimensions and tissue inhomogeneities on dosimetry planning of ${ }^{192}$ Ir HDR breast brachytherapy: a Monte Carlo dose verification study," Int J Radiat Oncol Biol Phys 61, 1596-1602 (2005).

${ }^{59}$ M.J. Rivard, L. Beaulieu, and F. Mourtada, "Enhancements to commissioning techniques and quality assurance of brachytherapy treatment planning systems that use model-based dose calculation algorithms," Med Phys 37, 2645-2658 (2010).

${ }^{60}$ S. Enger, G. Landry, M. D'Amours, F. Verhaegen, L. Beaulieu, M. Asai, and J. Perl, "Layered Mass Geometry: a novel technique to overlay seeds and applicators onto patient geometry in Geant4 brachytherapy simulations," Phys Med Biol XX, xX-yy (2012).

${ }^{61} \mathrm{O}$. Chibani, "Electron depth-dose distributions in water, Iron and Lead - the GEPTS system," Nucl Instrum Meth B 101, 357-378 (1995).

${ }^{62} \mathrm{H}$. Hedtjarn, G.A. Carlsson, and J.F. Williamson, "Accelerated Monte Carlo based dose calculations for brachytherapy planning using correlated sampling," Phys Med Biol 47, 351376 (2002).

${ }^{63}$ J. Dolan, Z. Lia, and J.F. Williamson, "Monte Carlo and experimental dosimetry of an ${ }^{125}$, brachytherapy seed," Med Phys 33, 4675-4684 (2006).

${ }^{64}$ E. Poon, Y. Le, J.F. Williamson, and F. Verhaegen, "BrachyGUI: an adjunct to an accelerated Monte Carlo photon transport code for patient-specific brachytherapy dose calculations and analysis," Journal of Physics: Conference Series 102, 012018 (2008). 
${ }^{65}$ G. Yegin, "A new approach to geometry modeling for Monte Carlo particle transport: An application to the EGS code system," Nuclear Instruments and Methods in Physics Research Section B: Beam Interactions with Materials and Atoms 211, 331-338 (2003).

${ }^{66}$ G. Yegin and D.W.O. Rogers, "A fast Monte Carlo code for multi-seed brachytherapy treatments including interseed effects," Med Phys 31, 1771(abs) (2004).

${ }^{67}$ S.A. Enger, A. Ahnesjö, F. Verhaegen, and L. Beaulieu, "Dose to tissue medium or water cavities as surrogate for the dose to cell nuclei at brachytherapy photon energies," Phys Med Biol 57, 4489 (2012).

${ }^{68}$ W. Schneider, T. Bortfeld, and W. Schlegel, "Correlation between CT numbers and tissue parameters needed for Monte Carlo simulations of clinical dose distributions," Phys Med Biol 45, 459-478 (2000).

${ }^{69}$ H.Q. Woodard and D.R. White, "The composition of body tissues," Br J Radiol 59, 1209-1218 (1986).

${ }^{70}$ R.L. Maughan, P.J. Chuba, A.T. Porter, E. Ben-Josef, and D.R. Lucas, "The elemental composition of tumors: kerma data for neutrons," Med Phys 24, 1241-1244 (1997).

${ }^{71}$ C. Xu, F. Verhaegen, D. Laurendeau, S.A. Enger, and L. Beaulieu, "An algorithm for efficient metal artifact reductions in permanent seed implants," Med Phys 38, 47-56 (2011).

${ }^{72}$ U. Schneider, E. Pedroni, and A. Lomax, "The calibration of CT Hounsfield units for radiotherapy treatment planning," Phys Med Biol 41, 111-124 (1996). 


\section{CHAPTER}

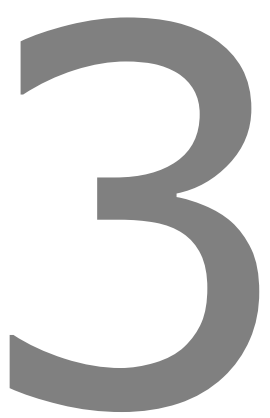

Sensitivity of low energy brachytherapy Monte Carlo dose calculations to uncertainties in human tissue composition

Guillaume Landry, Brigitte Reniers, Lars Murrer, Ludy Lutgens, Esther Bloemen-Gurp, Jean-Philippe Pignol, Brian Keller, Luc Beaulieu, Frank Verhaegen

Published in: Medical Physics 37(10):5188-5198, 2010. 


\section{ABSTRACT}

Purpose The objective of this work is to assess the sensitivity of Monte Carlo (MC) dose calculations to uncertainties in human tissue composition for a range of low photon energy brachytherapy sources: ${ }^{125} \mathrm{I},{ }^{103} \mathrm{Pd},{ }^{131} \mathrm{Cs}$ and an electronic brachytherapy source (EBS). The low energy photons emitted by these sources make the dosimetry sensitive to variations in tissue atomic number due to the dominance of the photoelectric effect. This work reports dose to a small mass of water in medium, $D_{w, m}$ as opposed to dose to a small mass of medium in medium, $D_{m, m}$.

Methods Mean adipose, mammary gland and breast tissues (as uniform mixture of the aforementioned tissues) are investigated as well as compositions corresponding to one standard deviation from the mean. Prostate mean compositions from three different literature sources are also investigated. Three sets of $M C$ simulations are performed with the GEANT4 code: (1) dose calculations for idealized TG-43-like spherical geometries using point sources. Radial dose profiles obtained in different media are compared to assess the influence of compositional uncertainties. (2) Dose calculations for four clinical prostate LDR brachytherapy permanent seed implants using ${ }^{125}$ I seeds (Model 2301, Best Medical, Springfield, VA). The effect of varying the prostate composition in the planning target volume (PTV) is investigated by comparing PTV $D_{90}$ values. (3) Dose calculations for four clinical breast LDR brachytherapy permanent seed implants using ${ }^{103} \mathrm{Pd}$ seeds (Model 2335, Best Medical). The effects of varying the adipose/gland ratio in the PTV and of varying the elemental composition of adipose and gland within one standard deviation of the assumed mean composition are investigated by comparing PTV $D_{90}$ values. For (2) and (3) the influence of using the mass density from CT scans instead of unit mass density is also assessed.

Results Results from simulation (1) show that variations in the mean compositions of tissues affect low energy brachytherapy dosimetry. Dose differences between mean and one standard deviation of the mean composition increasing with distance from the source are observed. It is established that the ${ }^{125} \mathrm{I}$ and ${ }^{131} \mathrm{Cs}$ sources are the least sensitive to variations in elemental compositions while ${ }^{103} \mathrm{Pd}$ is most sensitive. Results from simulation (2) show that two prostate compositions are dosimetrically equivalent to water while the third shows $D_{90}$ differences of up to $4 \%$. Results from simulation (3) show that breast is more sensitive than prostate with dose variations of up to $30 \%$ from water for $70 \%$ adipose/ $30 \%$ gland breast. The variability of the breast composition adds a $\pm 10 \%$ dose variation.

Conclusion Low energy brachytherapy dose distributions in tissue differ from water and are influenced by density, mean tissue composition and patient-to-patient composition variations. Our results support the use of a dose calculation algorithm accounting for heterogeneities such as MC. Since this work shows that variations in mean tissue compositions affect MC dosimetry and result in increased dose uncertainties, we conclude that imaging tools providing more accurate estimates of elemental compositions such as dual energy CT would be beneficial. 


\section{INTRODUCTION}

Low dose rate (LDR) transperineal permanent seed implantation performed under transrectal ultrasound (TRUS) guidance is widely used in the treatment of early stage prostate $_{\text {cancer }}{ }^{1,2}$. Given the demonstrated dose-outcome relationship ${ }^{3}$ for such implants, accurate dosimetry is necessary. Seed implants have also recently been used for partial breast irradiation of early stage breast cancer patients ${ }^{4-6}$ following breastconserving surgery.

LDR brachytherapy seed implant dosimetry currently follows the AAPM Radiation Therapy Committee Task Group 43 formalism ${ }^{7,8}$ (TG-43). The TG-43 protocol utilizes dose kernels obtained in uniform water spheres of $15 \mathrm{~cm}$ radius centered on a brachytherapy seed. This approach is an approximation of the patient geometries encountered in clinical implants and has its shortcomings. Since the photoelectric effect is the dominant interaction mode for the low energy photons emitted by isotopes such as ${ }^{103} \mathrm{Pd},{ }^{125} \mathrm{I}$, and ${ }^{131} \mathrm{Cs}$ or by electronic brachytherapy (EBS) sources, tissue heterogeneities and interseed attenuation influence dosimetry ${ }^{9-16}$. Post-surgical clips and prostate calcifications also may have an influence. Furthermore, the continuous low energy spectrum of EBS sources is subject to a non-negligible spectral hardening effect.

The AAPM Radiation Therapy Committee Task Group 186 (TG-186) has been mandated to investigate model based dose calculation (MBDC) algorithms capable of addressing the shortcomings of TG-43. Monte Carlo (MC) simulations have been proposed as one of the alternatives for their ability to accurately transport low energy photons in realistic geometries ${ }^{13}$. To achieve maximum accuracy in this energy range, $M C$ methods and other model-based algorithms require voxel by voxel knowledge of mass density $\rho$ and elemental composition. The use of modern clinical CT scanners can provide accurate density information using scanner specific mass (or electron) densityHounsfield Units (HU) calibration curves ${ }^{17-19}$. However, factors such as beam hardening, position in phantom, scanner diameter and beam energy have an influence on accuracy. ${ }^{19-21}$ Accurate elemental composition extraction is more challenging as many tissues with different compositions possess similar $\mathrm{HU}$ values ${ }^{22}$. Various schemes ${ }^{23-25}$ have been developed to extract compositions from CT data and inaccuracies incurred by this procedure have been evaluated ${ }^{26}$. In general the elemental compositions assigned to voxels are obtained from population-averaged data sets ${ }^{27-32}$ and ignore individual variability. Such variability introduces an uncertainty in human tissue composition, which has the potential to influence brachytherapy dosimetry for individual patients at low photon energies.

The objective of this work is to assess the influence of variations in the elemental composition of human tissues on the MC dosimetry of various low energy brachytherapy photon sources. Idealized TG-43-like spherical geometries are studied as well as clinical seed implants for breast and prostate LDR brachytherapy patients.

MBDC algorithms such as MC simulations allow dose calculations in various media and naturally provide dose to the local medium with transport in medium, $D_{m, m}$ and 
can also provide dose to a small mass of water with transport in medium, $D_{w, m}$. There is debate in the community over which approach is preferable. For this work the latter scoring scheme has been selected. This should not be interpreted as support by the authors for using $D_{w, m}$ over $D_{m, m}$.

\section{MATERIALS AND METHODS}

\section{Monte Carlo code and dose analysis tool}

GEANT4 ${ }^{33}$ is a general purpose MC simulation toolkit. Version 9.3 was used for this work with the photoelectric effect, Compton scattering and Rayleigh scattering processes of the Lawrence Livermore National Laboratory low energy electromagnetic model activated. This model exploits the EPDL ${ }^{34}, \mathrm{EEDL}^{35}$ and EADL ${ }^{36}$ evaluated data libraries. Since the range of secondary electrons is negligible for the energy regime of interest, dose was approximated by collision kerma and electrons were not tracked. There is lack of consensus in the field regarding the reporting of dose from photons transported in medium and scored in medium, $D_{m, m}$, vs. dose from photons transported in medium and scored in water, $D_{w, m}{ }^{37-39}$. This problem has not been addressed satisfactorily for low-energy brachytherapy. Ideally both quantities would be reported, but for clarity's sake the choice was made to report only one. $D_{w, m}$ was scored using a track length estimator and mass energy absorption coefficients of water were calculated at $1 \mathrm{keV}$ intervals using the EGSnrc ${ }^{40}$ (version V4-r2-3-1) user-code g. When using a track length estimator the $D_{w, m}$ (approximated by the collision kerma to water in medium, $\left.K_{w, m}\right)$ deposited in a scoring region of arbitrary shape by a photon $j$ is given by:

$$
D_{\mathrm{w}, \mathrm{m}}=K_{\mathrm{w}, \mathrm{m}}=\frac{E_{j}\left(\mu_{\mathrm{en}} / \rho\right)_{\mathrm{w}} I_{j}}{V}
$$

where $E_{j}$ is the photon's energy, $l_{j}$ is the straight line distance travelled inside the scoring regions of volume $V$ and $\left(\mu_{\mathrm{en}} / \rho\right)_{w}$ is the mass energy absorption coefficient of water at $E_{j}^{41}$. This method of scoring is used in this study for two types of scoring regions. For the idealized geometries spherical shells are used while grids of voxels are used for the clinical cases.

BrachyGUI ${ }^{42,43}$ is a brachytherapy dose analysis and planning tool developed with MATLAB (version 7.9.0, MathWorks, Natick, MA). It was used in this work to process DICOM data and to create input files for GEANT4 patient simulations as well as to analyze dose distributions.

\section{Composition of human tissues}

There are various literature sources ${ }^{27-32}$ reporting elemental compositions of human tissues. All report mean $(M)$ compositions of the main tissue types while a 
$\mathrm{few}^{28,30,31}$ also provide a range of compositions corresponding to the standard deviation $(\sigma)$ for a given tissue type. Since most sources cite Woodard ${ }^{28}$ when discussing the range of compositions, we based the majority of our work on the compositions reported in that paper. For a given tissue type three compositions are used; $M-\sigma, M$ and $M$ $+\sigma$ and are indexed as $h i-Z$, mean- $Z$ and $l o-Z$. The indexing is based on the value of the effective atomic number $Z_{\text {eff, }}$ given by:

$$
Z_{\text {eff }}=\left(\sum_{i} w_{i} Z_{i}^{3.5}\right)^{1 / 3.5}
$$

where $w_{i}$ is the mass fraction of element $i$ with atomic number $Z_{i}{ }^{44}$ Prostate compositions varying from one report to the other were also included and are indexed $A, B, C$. The compositions studied and their sources are reported in Table 3.1. The tissues listed were chosen because they represent tissues found in clinical sites where low-energy brachytherapy is a treatment option. Mammary gland tissue is labeled as Gland.

Table 3.1: Elemental composition of human tissues and water investigated in this work. Unless indicated otherwise, compositions are from Woodard and White ${ }^{28}$.

\begin{tabular}{|c|c|c|c|c|c|c|c|}
\hline \multirow[t]{2}{*}{ Tissue } & $\mathrm{H}$ & C & $\mathrm{N}$ & $\mathrm{O}$ & $Z>8$ & $\begin{array}{l}\text { Mass } \\
\text { density }\end{array}$ & $Z_{e f f}$ \\
\hline & \multicolumn{5}{|c|}{ \% by weight } & \multicolumn{2}{|l|}{$\mathrm{g} \cdot \mathrm{cm}^{-3}$} \\
\hline Adipose hi-Z & 11.2 & 51.7 & 1.3 & 35.5 & $\begin{array}{l}\mathrm{Na}(0.1), \\
\mathrm{Cl}(0.1)\end{array}$ & 0.970 & 6.84 \\
\hline Adipose mean-Z & 11.4 & 59.8 & 0.7 & 27.8 & $\begin{array}{l}\mathrm{Na}(0.1), \quad \mathrm{S}(0.1) \\
\mathrm{Cl}(0.1)\end{array}$ & 0.950 & 6.67 \\
\hline Adipose lo-Z & 11.6 & 68.1 & 0.2 & 19.8 & $\begin{array}{l}\mathrm{Na}(0.1), \quad \mathrm{S}(0.1) \\
\mathrm{Cl}(0.1)\end{array}$ & 0.930 & 6.47 \\
\hline Gland hi-Z & 10.2 & 15.8 & 3.7 & 69.8 & $\begin{array}{l}\mathrm{Na}(0.1), \quad \mathrm{P}(0.1) \\
\mathrm{S}(0.2), \mathrm{Cl}(0.1)\end{array}$ & 1.060 & 7.58 \\
\hline Gland mean-Z & 10.6 & 33.2 & 3.0 & 52.7 & $\begin{array}{l}\mathrm{Na}(0.1), \quad \mathrm{P}(0.1) \\
\mathrm{S}(0.2), \mathrm{Cl}(0.1)\end{array}$ & 1.020 & 7.27 \\
\hline Gland lo-Z & 10.9 & 50.6 & 2.3 & 35.8 & $\begin{array}{l}\mathrm{Na}(0.1), \quad \mathrm{P}(0.1) \\
\mathrm{S}(0.1), \mathrm{Cl}(0.1)\end{array}$ & 0.990 & 6.89 \\
\hline Prostate A & 10.5 & 8.9 & 2.5 & 77.4 & $\begin{array}{l}\mathrm{Na}(0.2), \quad \mathrm{P}(0.1) \\
\mathrm{S}(0.2), \mathrm{K}(0.2)\end{array}$ & 1.040 & 7.76 \\
\hline Prostate B* & 9.76 & 9.11 & 2.47 & 78.1 & $\begin{array}{l}\mathrm{Na}(0.21), \quad \mathrm{P}(0.1), \\
\mathrm{Mg}(0.019), \mathrm{K}(0.2), \\
\mathrm{Zn}(0.008), \\
\mathrm{Ca}(0.023)\end{array}$ & 1.045 & 7.76 \\
\hline Prostate $\mathrm{C}^{+}$ & 10.5 & 25.6 & 2.7 & 60.2 & $\begin{array}{ll}\mathrm{Na}(0.1), & \mathrm{P}(0.2), \\
\mathrm{S}(0.3), & \mathrm{Cl}(0.2), \\
\mathrm{K}(0.2) & \end{array}$ & 1.040 & 7.60 \\
\hline Water & 0.112 & 0 & 0 & $\begin{array}{l}0.88 \\
8 \\
\end{array}$ & 0 & 1.000 & 7.73 \\
\hline
\end{tabular}

*ICRP $(1975)^{27}$

+ICRP $(2002)^{32}$

Female breast tissue, which can be modeled as being composed of adipose and mammary gland tissue, has also been studied in this work. The mass fraction of both 
tissues in the female breast is known to vary from one individual to the other ${ }^{45}$ and to influence low energy brachytherapy dosimetry ${ }^{14,16}$. We modeled breast tissue as a uniform mixture of adipose and mammary gland tissue and three mass fractions (Adipose/mammary Gland) were studied: A30/G70, A50/G50 and A70/G30. This range approximates the reported range of breast composition in women undergoing mammography ${ }^{46}$. For each $A / G$ mixture the nine possible combinations of adipose and mammary gland tissue compositions in Table I were investigated.

Given the difficulty of accurately extracting tissue composition from CT data and the practice of assigning mean tissue compositions in $M C$ simulations, the variations in tissue compositions introduced above can be interpreted as a compositional uncertainty.

\section{Simulations in uniform geometries}

A GEANT4 application was designed to transport photons emitted from isotropic point sources in $100 \mathrm{~cm}$ radius spheres of uniform medium. Such a large radius was chosen to simulate an infinite geometry and provide full backscatter. Dose to water per emitted photon was scored in $1 \mathrm{~mm}$ thick spherical shells centered at every millimeter for photons transported in both medium and water (using the track length estimator). The application was benchmarked by running mono energetic simulations in water and results were compared to in-house EGSnrc MC simulations using the C++ class library ${ }^{47}$ and published Penelope (v. 2001) ${ }^{48} \mathrm{MC}$ results $^{49}$. Sub percent agreement was obtained between all codes.

Photon spectra used for the point sources were obtained by fully simulating four brachytherapy sources: a model $2301{ }^{125}$ I source and a model $2335{ }^{103} \mathrm{Pd}$ source (Best Medical, Springfield, VA), a model Cs-1Rev2 ${ }^{131}$ Cs source (IsoRay Medical, Richland, WA) and an Axxent EBS (Xoft, Sunnyvale, CA) source. The ${ }^{125} \mathrm{I},{ }^{103} \mathrm{Pd}$ and ${ }^{131} \mathrm{Cs}$ sources were modeled in GEANT4 according to published descriptions ${ }^{50,51}$. Radial dose functions for these three sources agreed with published results ${ }^{50,51}$ within $\pm 1.5 \%$ over the 0 to $8 \mathrm{~cm}$ range and dose rate constants within $0.5 \%$. Anisotropy functions also showed good agreement with absolute differences averaged over the 0 to 90 degree and 0 to 4 $\mathrm{cm}$ range of $1 \pm 2 \%$ (absolute standard deviation), $1 \pm 1 \%$ and $1 \pm 1 \%$ while the maximum deviations were $8 \%, 2.3 \%$ and $7 \%$ for the ${ }^{125} \mathrm{I},{ }^{103} \mathrm{Pd}$ and ${ }^{131} \mathrm{Cs}$ sources respectively. Deviations above $3 \%$ were only observed at 0 degree where volume averaging effects are more severe. Phase space files containing the photon distribution were collected at the surfaces of each source and photon spectra accounting for the spectral shift caused by the encapsulation were extracted from them. The spectra were collected over the whole phase space file, averaging out the source anisotropy. They were cut below $5 \mathrm{keV}$, neglecting the $4.5 \mathrm{keV}$ characteristic $\mathrm{x}$-rays produced in the titanium encapsulation, which only affects the dose in the first $\mathrm{mm}$ from the source. The Axxent source operating at $50 \mathrm{kV}$ with its cooling water sheath was simulated with GEANT4 and its emission spectrum was extracted following the same approach ${ }^{52}$. 
Each of these spectra was used to emit photons from the point source, ignoring anisotropy. The mean photon energies for model $2335\left({ }^{103} \mathrm{Pd}\right)$, model $2301\left({ }^{125} \mathrm{I}\right)$, model Cs-1Rev2 $\left({ }^{131} \mathrm{Cs}\right)$ and $50 \mathrm{kV}$ Axxent emission spectra were $21.0 \mathrm{keV}, 28.4 \mathrm{keV}$, $30.4 \mathrm{keV}$ and $26.9 \mathrm{keV}$ respectively. Absolute radial dose distributions were obtained for tissue compositions by simulating $10^{6}$ photon histories ensuring relative statistical uncertainty less than $1 \%$ (calculated according to Walters ${ }^{53}$ ) at $10 \mathrm{~cm}$. The dose distribution obtained by transporting photons in water and scoring dose to water, $D_{w, w}$, represents a close approximation of the TG-43 protocol. The approximation consists of using a point source instead of a real brachytherapy source. The fact that the water volume in our work simulates infinity as opposed to the $15 \mathrm{~cm}$ radius used in TG-43 is of no consequence for doses of interest, which are in the first $0-8 \mathrm{~cm}$ and are not influenced by scatter beyond $15 \mathrm{~cm}$, given the low energies at play ${ }^{54}$. Therefore, $D_{w, w}$ will be labeled as $D_{\mathrm{TG}-43}$.

\section{Clinical site 1: prostate}

MC dose calculations were performed for four prostate cancer patients who underwent transperineal implantation of the model $2301{ }^{125} \mathrm{I}$ source under TRUS guidance at the MAASTRO Clinic where a $145 \mathrm{~Gy}$ prescription dose is given to the ultrasound prostate contour, taken as the planning target volume (PTV). Implant description for each patient is presented in Table 3.2. CT scans with $3 \mathrm{~mm}$ slice thickness taken within $24 \mathrm{hrs}$ of implantation were imported in the VariSeed planning system (Varian, Palo Alto, CA) where prostate contours were drawn by a radiation oncologist and seed positions established using VariSeed's seed finder algorithm. DICOM data exported from VariSeed were imported in BrachyGUI where material and density assignment were established for each voxel. Two simulations were prepared with BrachyGUI. In the first one $\left(\mathrm{MC} \rho_{=1}\right)$, the density of every voxel was set to $1 \mathrm{~g} \cdot \mathrm{cm}^{-3}$ while in the second one ( $\mathrm{MC} \rho_{=\mathrm{CT}}$ ) mass density maps were established according to the CT scanner's $\mathrm{HU}-\rho$ calibration curve. It is not possible to assign continuous densities in GEANT4 so density values were discretized in $0.01 \mathrm{~g} \cdot \mathrm{cm}^{-3}$ intervals. In all simulations the interseed effect ${ }^{10,12-14}$ was ignored; in $\mathrm{MC} \rho_{=\mathrm{CT}}$ simulations this was done by replacing the mass density of the seeds by the mean mass density of surrounding tissue. This was done to provide clear insight on the influence of tissue composition and also because the interseed effect has been studied elsewhere. In both types of simulations, phase space files were used and the geometry did not contain a model of the brachytherapy seed, again to neglect the interseed attenuation. In these simulations the sources' anisotropy was preserved.

For both $\mathrm{MC} \rho_{=1}$ and $\mathrm{MC} \rho_{=\mathrm{C} T}$ simulations, Prostate $A, B$ and $C$ as well as Water compositions were assigned to voxels within the prostate contour. Voxels outside the prostate contour were modeled as water. The CT data was resampled on a $2 \mathrm{~mm} X$ $2 \mathrm{~mm} \times 3 \mathrm{~mm}$ (slice thickness is $3 \mathrm{~mm}$ ) voxel grid and a phase space file containing the anisotropic photon distribution of the seed was used as photon source at every seed position. Seeds were assumed parallel to the direction of implantation. The $D_{w, m}$ in each voxel was obtained using the track length estimator. The dose distributions 
obtained from $\mathrm{MC} \rho_{=1}$ simulations using the Water composition are conceptually equivalent to the TG-43 formalism and will be labeled $D_{\text {TG-43. The number of histories }}$ $\left(5 \times 10^{7}\right)$ yielded a statistical (type A) dose uncertainty smaller than $1 \%$ in the prostate contour. Dose distributions were imported in BrachyGUI where the minimal dose to $90 \%$ of the prostate contour volume, $D_{90}$, was obtained from dose volume histogram analysis for each composition assigned to the PTV and for each simulation type. $D_{90}$ is of interest given its correlation with outcome ${ }^{3}$. The $D_{90}$ calculated by BrachyGUI for the $D_{\text {TG-43 }}$ distribution and the $D_{90}$ reported by VariSeed for a clinical implant using our TG43 parameters for the model $2301{ }^{125} \mathrm{I}$ source agreed within $2 \%$. The absolute type A uncertainty on $D_{90}$ is estimated to be $0.2 \%$ (percentage of prescribed dose) and was assessed by running ten batches of photons and obtaining the standard deviation of the distribution of $D_{90}$ values.

Table 3.2: Description of prostate implants with ${ }^{125}$ / seeds.

\begin{tabular}{lllll}
\hline \hline & \multicolumn{3}{l}{ Patient number } \\
\cline { 2 - 5 } & 1 & 2 & 3 & 4 \\
\hline Prescription dose (Gy) & 145 & 145 & 145 & 145 \\
Seed activity (U*) & 0.596 & 0.603 & 0.603 & 0.603 \\
Prostate volume (cc) & 54.8 & 31.8 & 27.3 & 83.6 \\
Number of seeds & 75 & 77 & 51 & 99 \\
\hline \hline
\end{tabular}

$* 1 \mathrm{U}=1 \mu \mathrm{Gy} \mathrm{m} \mathrm{h}^{-1}$

\section{Clinical site 2: breast}

Dose calculations were performed for four breast cancer patients from the Sunnybrook Health Sciences Centre (Toronto, Canada) who underwent permanent breast seed implants ${ }^{4-6}$ using the model $2335{ }^{103} \mathrm{Pd}$ seed. A $90 \mathrm{~Gy}$ dose was prescribed, covering the PTV which consists of "the lumpectomy cavity plus a margin of $1 \mathrm{~cm}$ modified to $5 \mathrm{~mm}$ deep to the skin surface and also along the fascia pectoralis" ${ }^{6}$. CT scans with $5 \mathrm{~mm}$ slice thickness taken 2 months post implantation with PTV contours drawn by a radiation oncologist were exported from VariSeed to BrachyGUI where $M C \rho_{=1}$ and $\mathrm{MC} \rho_{=\mathrm{CT}}$ simulations were prepared. Voxels within the PTV contour were assigned each of the breast compositions described in Table 3.3 as well as Water. For a given adipose/gland ratio, the compositions labeled $h i-Z$ and $l o-Z$ were chosen to illustrate the largest possible variations from A50/G50 mean- $Z$ attainable from the selection of compositions used in this work. The dose calculation grid used for these cases was $1 \mathrm{~mm} \times 1 \mathrm{~mm} \times 5 \mathrm{~mm}$ (slice thickness is $5 \mathrm{~mm}$ ). BrachyGUI was used to extract the PTV $D_{90}$. Again $5 \times 10^{7}$ photons histories were simulated yielding type A relative uncertainties within the PTV lower than 1\%. Implant specific details are in Table 3.4. 
Table 3.3: Description of the breast compositions used for patient dose calculations.

\begin{tabular}{lllll}
\hline \hline Composition & $\begin{array}{l}\text { Adipose } \\
\text { fraction } \\
\text { (\% mass) }\end{array}$ & $\begin{array}{l}\text { Adipose } \\
\text { composition }\end{array}$ & $\begin{array}{l}\text { Gland } \\
\text { composition }\end{array}$ & $Z_{\text {eff }}$ \\
\hline A30/G70 hi-Z & 30 & Adipose hi-Z & Gland hi-Z & 7.38 \\
A30/G70 mean-Z & 30 & Adipose mean-Z & Gland mean-Z & 7.10 \\
A50/G50 mean-Z & 50 & Adipose mean-Z & Gland mean-Z & 6.99 \\
A70/G30 mean-Z & 70 & Adipose mean-Z & Gland mean-Z & 6.86 \\
A70/G30 lo-Z & 70 & Adipose lo-Z & Gland lo-Z & 6.60 \\
\hline \hline
\end{tabular}

Table 3.4: Description of breast implants with ${ }^{103} P d$ seeds.

\begin{tabular}{lllll}
\hline \hline & \multicolumn{4}{l}{ Patient number } \\
\cline { 2 - 5 } & 1 & 2 & 3 & 4 \\
\hline Prescription dose (Gy) & 90 & 90 & 90 & 90 \\
Seed activity (U) & 2.5 & 2.7 & 2.7 & 2.5 \\
PTV volume (cc) & 43.8 & 47.5 & 30.9 & 18.7 \\
Number of seeds & 64 & 78 & 60 & 49 \\
\hline \hline
\end{tabular}

\section{RESULTS AND DISCUSSION}

\section{Radial dose functions for single sources in idealized geometries}

Radial dose profiles reported below for the various tissue types under investigation are given in units of absolute dose (Gy) per emitted particle. The inverse square law dependence associated with the point source geometry has been factored out by multiplying dose values by $r^{2}$, where $r$ is the radial distance from the source to the scoring shell's mid-radius. Furthermore, the influence of tissue density has been removed by showing dose profiles as functions of radiological distance $\rho r$. The rationale is that a hypothetical treatment planning system using dose kernels $D_{w, m}$ calculated in medium could accurately extract density information from CT data ${ }^{17-19}$ and scale the distributions accordingly. The challenge of such a TPS would then reside in correctly assigning elemental composition to tissues from CT or other imaging data. In addition to dose profiles, ratios of doses to liquid water $\left(D_{\mathrm{TG}-43}\right)$ are reported to illustrate the magnitude of compositional variations compared to TG-43. Since these ratios are also plotted against the radiological distance they show the variability of tissues with unit mass density. It was observed that the ${ }^{131} \mathrm{Cs}$ source shows little difference when compared to the ${ }^{125} \mathrm{I}$ source because of their similar mean energy. Therefore results for the ${ }^{131} \mathrm{Cs}$ source are mentioned only sporadically in this section. 


\section{Adipose tissue}

Figure 3.1 shows dose profiles and dose ratios to $D_{\mathrm{TG}-43}$ for the three adipose tissue compositions of interest. We observe that the different attenuation between the three adipose compositions and water result in increasing differences from $D_{\mathrm{TG}-43}$ as one moves away from the source. We notice that the largest variation with distance is obtained for the ${ }^{103} \mathrm{Pd}$ isotope. This is due to the lower mean photon energy $(21.0 \mathrm{keV})$ of this source resulting in increased importance of the photoelectric cross-section. Figure 3.2(a) shows the ratio of the total mass attenuation coefficient $\mu / \rho$ of mean adipose tissue over water ${ }^{55}$, which illustrates that for the energies of interest (above $10 \mathrm{keV})$, the lower the energy, the larger the attenuation differences between media. Figure 3.1(b) shows that very close to the source, $D_{w, m} / D_{\mathrm{TG}-43}$ for all sources converge to unity since the attenuation becomes negligible.

To determine which source's dose distribution is the least sensitive to variations in medium, the relative dose variation $\Delta D$ caused by the compositional range of adipose tissue is plotted as a function of distance in Figure 3.2(b). The relative dose variation $\Delta D$ is given by:

$$
\Delta D=\frac{D_{\text {loz }}-D_{\mathrm{hiz}}}{D_{\text {mean z }}} \cdot 100 \% .
$$

We see that the most robust source within $5 \mathrm{~g} \cdot \mathrm{cm}^{-2}$ is ${ }^{125}$ I (and ${ }^{131} \mathrm{Cs}$, not shown) with deviations less than $20 \%$ while the least robust is ${ }^{103} \mathrm{Pd}$ with deviations above $35 \%$. The Axxent source shows smaller deviations beyond $5 \mathrm{~g} \cdot \mathrm{cm}^{-2}(\sim 20 \%)$ due to considerable spectral hardening [Figure 3.3(a)].
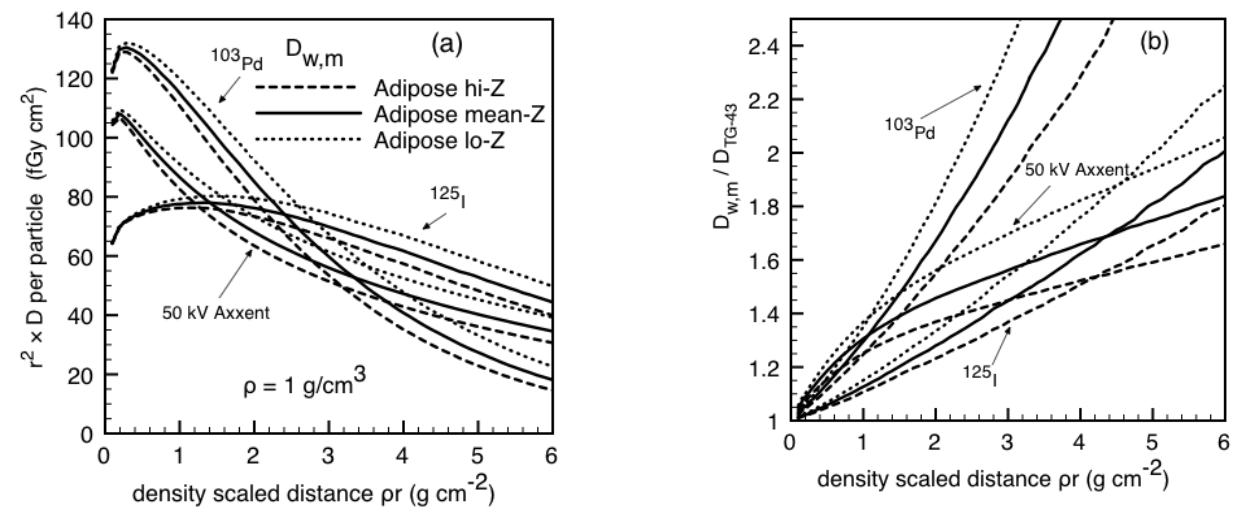

Figure 3.1: (a) Radial $D_{w, m}$ in three different compositions of adipose tissues corrected for the inverse square law for three different brachytherapy photon sources. (b) Ratio of $D_{w, m}$ to the dose obtained in water $\left(D_{T G-43}\right)$.

In general the compositional variability of adipose tissue has a second order influence when compared to the variation between mean adipose tissue (Adipose mean-Z) and water [Figure 3.1(b)]. 

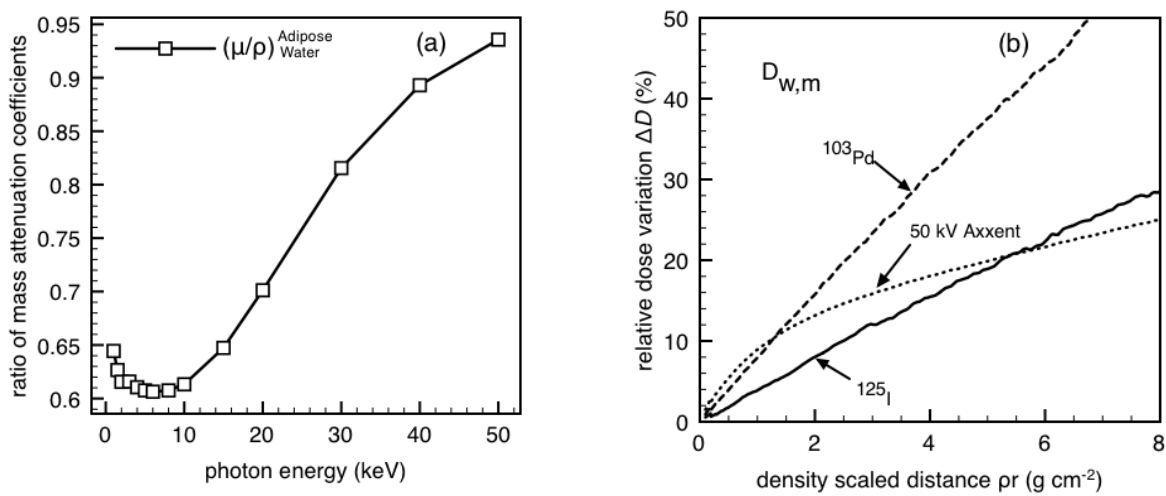

Figure 3.2: (a) Ratio of mass attenuation water to Adipose mean-Z as a function of photon energy. (b) Relative dose variation caused by variations in the composition of adipose tissue relative to the mean composition of adipose tissue.

From Figure 3.1(b) we notice that the $50 \mathrm{kV}$ Axxent source dose ratios exhibit a decreasing slope between 0 and $2 \mathrm{~g} \cdot \mathrm{cm}^{-2}$ followed by a constant slope beyond $2 \mathrm{~g} \cdot \mathrm{cm}^{-2}$. This is attributed to the soft continuous photon spectrum of the Axxent source, which undergoes significant hardening with depth. The photon fluence $\phi(E)$ was scored at various depths in both Water and Adipose mean- $Z$ and the fluence weighted mean photon energy $\langle E\rangle_{\phi(E)}[$ Figure 3.3(a)] as well as the fluence weighted mean photoelectric mass attenuation coefficient $\langle\tau / \rho(E)\rangle_{\phi(E)}[$ Fig. 3(b)] were calculated. Figure 3.3(a) shows that beam hardening is more severe in the first $2 \mathrm{~g} \cdot \mathrm{cm}^{-2}$ and more important for water, given the higher attenuation. Figure $3.3(\mathrm{~b})$ shows that the $\langle\tau / \rho(E)\rangle_{\phi(E)}$ ratio changes rapidly in the first $2 \mathrm{~g} \cdot \mathrm{cm}^{-2}$ and levels off beyond. This rapid variation is responsible for the changing slope observed in the dose ratios since the photoelectric effect is mainly responsible for dosimetric differences between tissues.
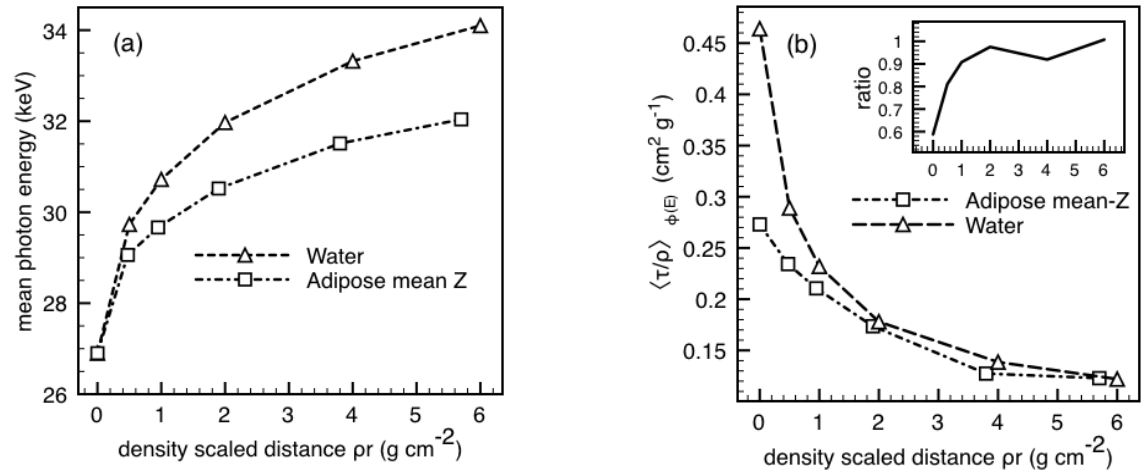

Figure 3.3: (a) Mean photon energy from $50 \mathrm{kV}$ Axxent source as a function of density scaled radial distance in Adipose mean-Z and Water showing different hardening. Data point at origin is for the unfiltered spectrum. (b) Mean photoelectric mass attenuation coefficient. (b, inset) Ratio on same abscissa showing stabilization with distance. 


\section{Mammary gland}

Results for mammary gland tissue are found in Figure 3.4. The mean composition (Gland mean-Z), having a $Z_{\text {eff }}$ closer to that of water, shows smaller dose differences from $D_{\mathrm{TG}-43}$ than mean adipose tissue. In general the same trends as for adipose tissue are observed although the larger $Z_{\text {eff }}$ variations between mammary gland compositions result in larger dose differences between mean- $Z$ and hi/lo- $Z$ compositions than observed in adipose [Figure 3.4(b)]. The dose differences between mean- $Z$ and hi/lo- $Z$ compositions are of the same order as the difference between Gland mean- $Z$ and Water.
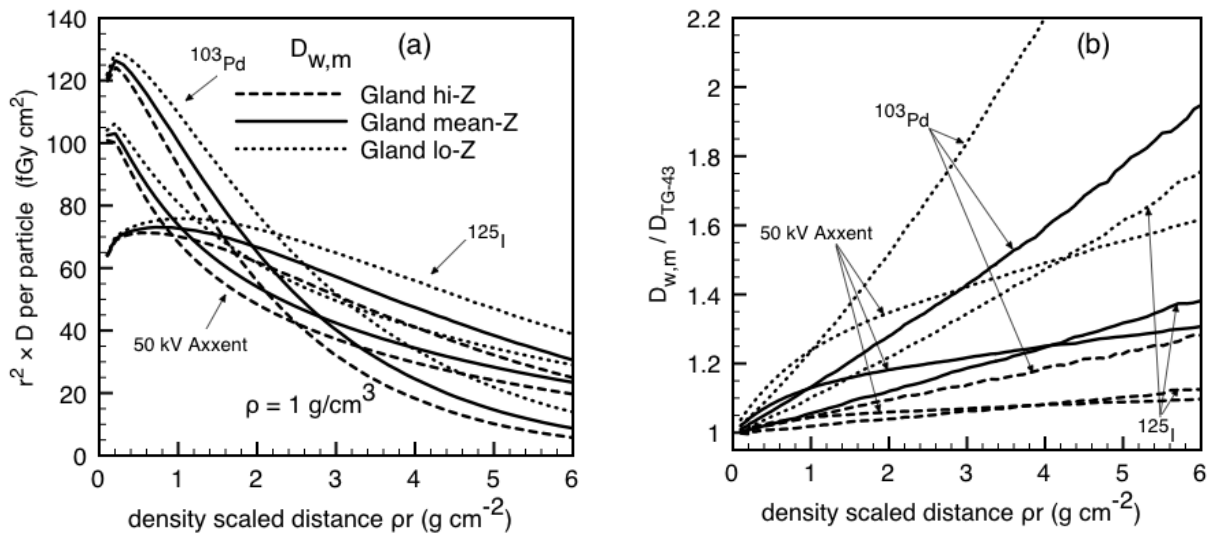

Figure 3.4: (a) Radial $D_{w, m}$ in three different compositions of mammary gland tissues corrected for the inverse square law for three different brachytherapy photon sources. (b) Ratio of $D_{w, m}$ to the dose obtained in water $\left(D_{T G-43}\right)$.

\section{Breast tissue}

Figure 3.5 shows $D_{w, m}$ data for breast tissue. For each adipose/gland mixture studied, the following combinations of adipose and glandular tissues are presented: Breast mean $-Z=$ Adipose mean-Z/Gland mean- $Z$, which represents the mean, Breast hi- $Z=$ Adipose hi-Z/Gland hi-Z and Breast lo-Z = Adipose lo-Z/Gland lo-Z which represent the largest variations from the mean. We observe that the higher the glandular content, the larger the variability between mean-Z and $h i / l o-Z$, as expected from the adipose and mammary gland results in previous sections. 

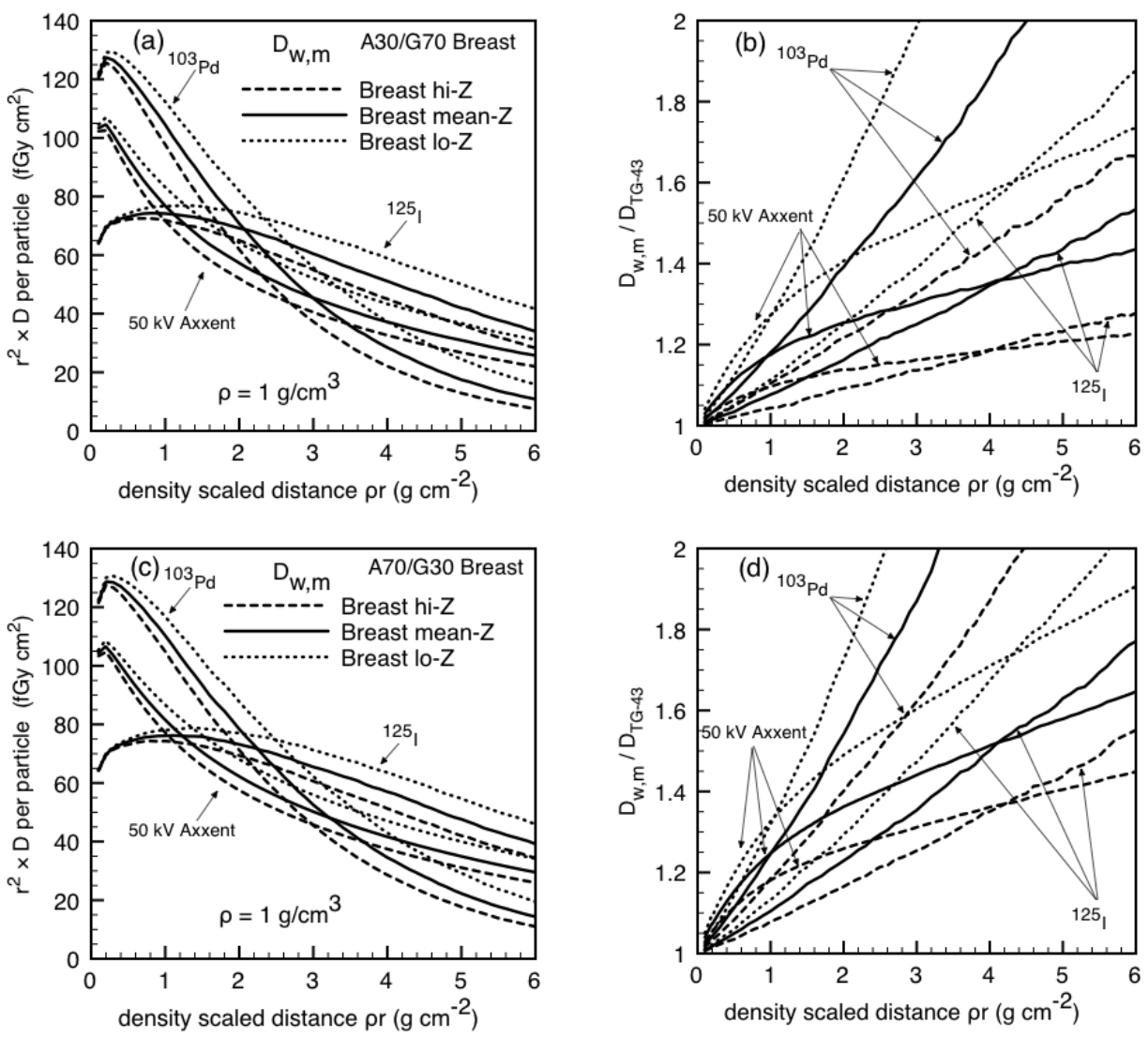

Figure 3.5: (a) Radial $D_{w, m}$ in $A 30 / G 70$ breast tissues corrected for the inverse square law for three different brachytherapy photon sources. The combinations of adipose and glandular tissue compositions were chosen to illustrate the maximum deviations. (b) Ratio of $D_{w, m}$ to the dose obtained in water $\left(D_{T G-43}\right)$. $(c, d)$ Same as above for A70/G30 breast.

\section{Prostate tissue}

The results for prostate tissues are presented in Figure 3.6. Prostate $B$ (see Table 3.1) has been determined to be dosimetrically equivalent to Prostate $A$ and has been omitted from the results. The $Z_{\text {eff }}$ values of both compositions are identical. It is interesting to note that Prostate $A$ is compositionally equivalent to water (neglecting density) at all energies while Prostate $C$ is not. Thus $D_{w, m} / D_{\mathrm{TG}-43}$ is about unity for Prostate $A$ and $B$ [Figure 3.6(b)]. ICRP Report $89^{32}$ provides the composition of Prostate $C$ and refers to ICRU Report $46^{30}$ as data source. The latter makes no mention of prostate tissue and it was determined that the composition reported by ICRP Report 89 for prostate is actually what ICRU Report 46 reports as "average male soft tissue 
(ICRU-44)". Whether Prostate A or Prostate C better represent mean prostate tissue requires further investigation. The difference between each prostate composition is expected to cause dose differences between Prostate $A$ and Prostate $C$ in clinical MC calculations with multiple sources.
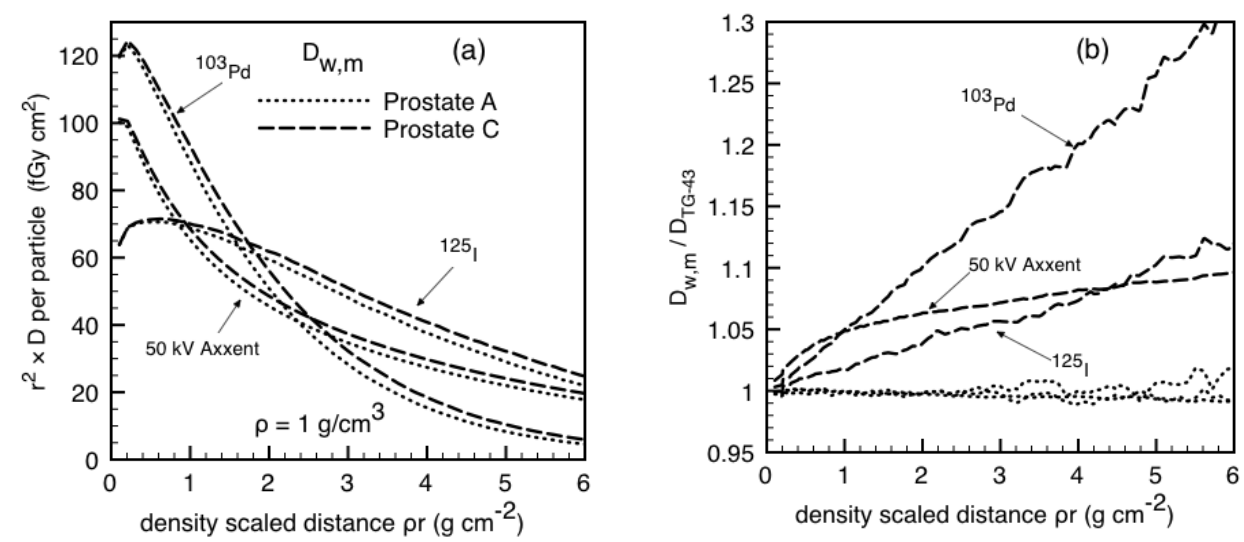

Figure 3.6: (a) Radial $D_{w, m}$ in two different compositions of prostate tissues corrected for the inverse square law for three different brachytherapy photon sources. (b) Ratio of each radial $D_{w, m}$ to the dose obtained in water $\left(D_{T G-43}\right)$. The curves Prostate $A$ for each source lie on top of each other close to unity.

\section{Prostate clinical dose calculations}

$M C \rho_{=1}$ results

The effect of compositional uncertainty on $\mathrm{MC} \rho_{=1} D_{w, m}$ calculations for clinical prostate cases is presented in Table 3.5 where $D_{90}$ values for the prostate PTV contour are presented as relative difference from $D_{\mathrm{TG}-43}$. The $D_{90}$ deviation, $\Delta D_{90}$, is given by:

$$
\Delta D_{90}=\frac{D_{90}^{\text {Comp }}-D_{90}^{\mathrm{TG}-43}}{D_{90}^{\mathrm{TG}-43}} \cdot 100 \%,
$$

where $D_{90}^{\text {Comp }}$ is the $D_{90}$ value for the composition of interest and $D_{90}^{\mathrm{TG}-43}$ is the value for the $D_{\text {TG-43 }}$ distribution. Averaged over the 4 patients there is a $3.2 \%$ difference between Prostate $A$ and Prostate $C$. Furthermore, Prostate $A$ is equivalent to Water (when neglecting density effects) within $0.5 \%$, as expected from the previous section. 
Table 3.5: Variations of prostate contour $D_{90}$ with various compositions compared to $D_{T G-43}$.

\begin{tabular}{|c|c|c|}
\hline $\begin{array}{l}\text { Dose calculation } \\
\text { method }\end{array}$ & $\begin{array}{l}\text { Mean } \Delta D_{90} \\
(\%)\end{array}$ & $\begin{array}{l}\text { Absolute } \\
\text { uncertainty } \\
\text { on } \Delta D_{90}(\%)\end{array}$ \\
\hline \multicolumn{3}{|l|}{$\mathrm{MC} \rho_{=1}$} \\
\hline Prostate A & 0.0 & 0.1 \\
\hline Prostate C & 3.2 & 0.3 \\
\hline \multicolumn{3}{|l|}{$\mathrm{MC} \rho_{=\mathrm{CT}}$} \\
\hline Water $\left(\rho_{\mathrm{CT}}\right)$ & -1.7 & 0.3 \\
\hline Prostate A & -2.0 & 0.2 \\
\hline Prostate C & 1.5 & 0.3 \\
\hline
\end{tabular}

$M C \rho_{=c T}$ results

The lower section of Table 3.5 shows that using densities obtained from CT data ( $\left.\mathrm{MC} \rho_{=\mathrm{CT}}\right)$ instead of unit density $\left(\mathrm{MC} \rho_{=1}\right)$ decreases $D_{90}$ values by $1-2 \%$ compared to $\mathrm{MC} \rho_{=1}$ results. Prostate tissue has a relative density larger than unity, which increases attenuation. The mean mass density in the prostate PTV contour was $1.05 \mathrm{~g} \cdot \mathrm{cm}^{-3}$ averaged over the four patients with a standard deviation of $0.02 \mathrm{~g} \cdot \mathrm{cm}^{-3}$. All patients had mean mass density of $1.05 \mathrm{~g} \cdot \mathrm{cm}^{-3}$.

Carrier et al. ${ }^{13}$ obtained $D_{90}$ values $2.6 \pm 0.4 \%$ lower for MC simulations in tissue compared to water. They scored $D_{m, m}$ and compared to simulations in water for 28 prostate patients using a composition equivalent to Prostate $B$. The difference of scoring scheme (their $D_{m, m}$ vs. our $D_{w, m}$ ) forbids direct comparison of our results. To provide a comparison the $D_{w, m}$ results were converted to $D_{m, m}$ using the $\mu_{\mathrm{en}} / \rho$ ratio Water to Prostate $A$ (equivalent to Prostate $B$ ) at the mean energy of the ${ }^{125}$ I source (28.4 keV). The $D_{90}$ deviation was recalculated using their equation:

$$
\Delta D_{90}^{\mathrm{m}, \mathrm{m}}=\left(1-\frac{D_{90}^{\mathrm{TG}-43}}{D_{90}^{\mathrm{m}, \mathrm{m}}}\right) \cdot 100 \%,
$$

where $D_{90}^{m, m}$ is now from the $D_{m, m}$ distribution. Using a value of 0.993 for the $\mu_{\mathrm{en}} / \rho$ ratio leads to $a D_{90}^{\mathrm{m}, \mathrm{m}}$ of $-1.3 \pm 0.2 \%$. This is a smaller effect than what was observed by Carrier et al. who looked at differences between simulations in tissue and water where the interseed attenuation effect is present. Our study isolates the effect of tissue composition from the interseed effect and only looks at 4 patients. Furthermore the use of the $\mu_{\mathrm{en}} / \rho$ ratio at the average energy of the initial spectrum could introduce an error by ignoring the variation of $\mu_{\mathrm{en}} / \rho$ over the spectrum's energy range as well as shifts in mean energy with depth in tissue. 
Our study left out the interseed effect but the literature ${ }^{10-13}$ shows a $D_{90}$ reduction of about $4 \%$, which is of the same order as the tissue effects presented in Table 3.5.

\section{Breast clinical dose calculations}

$M C \rho_{=1}$ results

The effects of compositional uncertainty on $\mathrm{MC} \rho_{=1} D_{w, m}$ calculations for breast clinical cases are presented in Table 3.6 where relative differences from $D_{\mathrm{TG}-43}$ for PTV $D_{90}$ values are found. The $D_{90}$ deviation, $\Delta D_{90}$, is given by equation 3 . The results of the 4 patients have been averaged. The effect of compositional uncertainty is derived by looking at the difference between mean- $Z$ and hi/lo- $Z$ labeled compositions. The variation for $A 30 / G 70$ breast ( $9 \%$ between mean-Z and $h i-Z$ ) and A70/G30 (9\% between $l o-Z$ and mean-Z) are the same. Such variations are of the same order as what is observed going from A30/G70 mean-Z to A70/G30 mean-Z (8\%). In general, all compositions studied have higher $D_{90}$ values than water because they all have lower $Z_{\text {eff }}$ values, resulting in lower attenuation, which in turn results in increased fluence and higher $D_{w, m}$ values.

Table 3.6: Variations of breast PTV D90 with various compositions compared to DTG-43.

\begin{tabular}{lll}
\hline $\begin{array}{l}\text { Dose calculation } \\
\text { method }\end{array}$ & $\begin{array}{l}\text { Mean } \Delta D_{90} \\
(\%)\end{array}$ & $\begin{array}{l}\text { Absolute } \\
\text { uncertainty on } \\
\Delta D_{90}(\%)\end{array}$ \\
\hline $\mathrm{MC} \rho_{=1}$ & \\
A30/G70 hi-Z & 12.6 & 0.9 \\
A30/G70 mean-Z & 22.1 & 1.4 \\
A50/G50 mean-Z & 26.3 & 1.8 \\
A70/G30 mean-Z & 30.4 & 2.3 \\
A70/G30 lo-Z & 39.8 & 3.2 \\
MC $\rho_{=C T}$ & & \\
Water ( $\left.\rho_{\text {CT }}\right)$ & 3.9 & 1.5 \\
A30/G70 hi-Z & 16.3 & 1.7 \\
A30/G70 mean-Z & 25.8 & 2.0 \\
A50/G50 mean-Z & 29.8 & 2.6 \\
A70/G30 mean-Z & 34.1 & 2.6 \\
A70/G30 lo-Z & 42.9 & 3.1 \\
\hline \hline
\end{tabular}

$M C \rho_{=c T}$ results

The effect of accounting for the CT density, obtained by comparing $\mathrm{MC} \rho_{=\mathrm{CT}}$ to $\mathrm{MC} \rho_{=1}$ results from the lower section of Table 3.6 for a given composition, is a $2-4 \%$ increase 
in $D_{90}$ explained by the lower average density of breast tissue on CT. The mean mass density in the breast PTV contour was $0.94 \mathrm{~g} \cdot \mathrm{cm}^{-3}$ averaged over the four patients with a standard deviation of $0.02 \mathrm{~g} \cdot \mathrm{cm}^{-3}$. A larger variation between patients was observed for breast cases with the highest mean density being $0.96 \mathrm{~g} \cdot \mathrm{cm}^{-3}$ and the lowest 0.92 $\mathrm{g} \cdot \mathrm{cm}^{-3}$.

By using the same approach described above to convert $D_{w, m}$ to $D_{m, m}$ our $\mathrm{MC} \rho_{=\mathrm{CT}}$ results can be compared to the work of Afsharpour et al. ${ }^{16}$ who studied the influence of varying the adipose/glandular ratio using mean-Z compositions for 5 breast patients implanted with the model $2335{ }^{103} \mathrm{Pd}$ seed. They looked at adipose mass fractions of $25 \%, 50 \%$ and $75 \%$ and compared $D_{m, m} D_{90}$ values for MC simulations in breast tissue and in water. Table 3.7 shows the comparison between their linearly interpolated results to $30 \%, 50 \%$ and $70 \%$ adipose mass fractions and our $\mathrm{MC} \rho_{=\mathrm{CT}} D_{w, m}$ results converted to $D_{m, m}$ using the $\mu_{\mathrm{en}} / \rho$ ratio at the ${ }^{103} \mathrm{Pd}$ source's spectrum average energy. Our $D_{90}$ deviation results have been recalculated using their expression:

$$
\Delta D_{90}^{\mathrm{m}, \mathrm{m}}=\left(\frac{D_{90}^{\mathrm{TG}-43}}{D_{90}^{\mathrm{m}, \mathrm{m}}}-1\right) \cdot 100 \%
$$

where $D_{90}^{m, m}$ is from the $D_{m, m}$ distribution. They fall within the range of values they observed, although they are systematically higher by a few percent.

Table 3.7: Comparison of breast PTV $D_{90}$ variations $M C_{\rho=C T}$ with the results of Afsharpour et al. ${ }^{16}$

\begin{tabular}{lll}
\hline \hline & \multicolumn{2}{l}{$\Delta D_{90}{ }^{m, m}(\%)$} \\
\cline { 2 - 3 } Adipose mass & This & Afsharpour \\
fraction (\%) & work & et al. \\
\hline 30 & $8 \pm 2$ & $6 \pm 4$ \\
50 & $11+2$ & $8 \pm 4$ \\
70 & $14 \pm 2$ & $11 \pm 6$ \\
\hline \hline
\end{tabular}

For breast cases composition effects overshadow the interseed attenuation, reported to correspond to a $3 \%$ decrease of $D_{90}{ }^{15,16}$.

Figure 3.7 presents ratios of dose distributions in an axial CT slice for patient 4 for the A70/G30 breast compositions, which is close to the average breast according to a recent study by Yaffe et $a f^{56}$ challenging the notion that A50/G50 breast is representative of the average. Their study looked at North American women who underwent mammography screening and they report a mean mammary gland volumetric percentage of $20 \%$ with $80 \%$ of women having gland volumetric percentage below $27 \%$. Whether this composition is representative of the average breast cancer patient requires further scrutiny.

First, the magnitude of dose variations obtained by going from $D_{\mathrm{TG}-43}$ to $\mathrm{MC} \rho_{=\mathrm{CT}}$ simulations in Breast mean-Z A70/G30 are presented [Figure 3.7(a)]. We observe a general increase in dose with hot spots of up to $30 \%$. Second, the magnitude of dose variations obtained by going from Breast mean-Z to Breast lo- $Z$ is presented [Fig- 
ure 3.7(b)]. Similar trends of reduced amplitude are observed. The variations are smaller than in Figure 3.7(a) but are still in the order of $10 \%$.

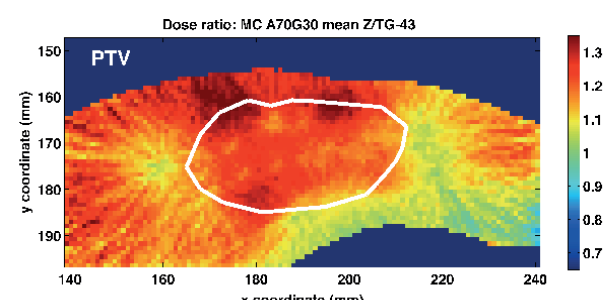

(a)

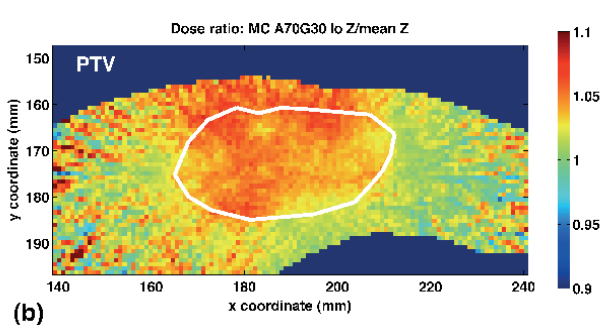

(b)

Figure 3.7: (a) Ratio of Breast mean-Z A70/G30 from a brachytherapy breast implant using ${ }^{103} \mathrm{Pd}$ seeds and $D_{T G-43}$. (b) Ratio of Breast lo-Z over Breast mean-Z.

The variations, or uncertainties, on adipose and mammary gland tissue compositions investigated for these patients represent one standard deviation according to the literature and thus would cover $66 \%$ of individuals. It is therefore expected that $34 \%$ of patients may exhibit even greater compositional and dosimetric variations.

In summary, replacing water by mean $A 70 / G 30$ breast leads to a $30 \% D_{90}$ difference from TG-43, a first order effect. The additional $10 \%$ variation attributed to uncertainties in mammary gland and adipose tissue compositions can be considered a second order effect while the effect of using the CT density ( $4 \%$ increase of $D_{90}$ ) and the interseed effect ( $3 \%$ decrease of $D_{90}$ ) can be labeled as third order effects.

\section{Scoring $D_{w, m}$ Vs $D_{m, m}$}

The lack of consensus in the field regarding the scoring of $D_{w, m}$ or $D_{m, m}$ probably stems from the fact that strong arguments are found on both sides. $D_{m, m}$ is the natural quantity obtained from MBDC algorithms and is more directly related to the energy deposited in the proper medium. Proponents of $D_{m, m}$ claim that it would provide an improved dose response relationship and that conversion schemes to $D_{w, m}$ introduce unnecessary uncertainty and potential for errors ${ }^{38}$.

The fact that dosimeters are generally calibrated in terms of absorbed dose to water may be seen as an argument in favor of $D_{w, m}$. Experimental validation of calculations reporting $D_{m, m}$ requires the dosimeter to be calibrated in terms of absorbed dose to every medium of interest and requires accurate knowledge of medium composition. Any uncertainty on the actual composition of the medium would translate into increased $D_{m, m}$ measurement uncertainty.

Still there is no indication that $D_{w, m}$ would provide a more accurate dose-effect relationship than $D_{m, m}$ and our choice of scoring method should not be interpreted as support for the use of $D_{w, m}$ over $D_{m, m}$ in brachytherapy dose reporting. 


\section{CONCLUSION}

The sensitivity of $M C$ dose calculations for low energy brachytherapy sources to uncertainties in human tissue compositions was assessed for both idealized geometries and real seed implants for $D_{w, m}$. MC simulations in TG-43 like geometries show that dose distributions in tissue are different than in water. Mean adipose tissue shows the largest difference from water while the variations on its composition represent a second order effect. For mean mammary gland tissue differences with water are smaller but the variation on its composition represents a first order effect. The various mean prostate compositions found in the literature give rise to dosimetric differences. The point source simulations indicate that ${ }^{125} \mathrm{I}$ and ${ }^{131} \mathrm{Cs}$ are more dosimetrically robust to compositional variations.

Patient simulations show that compositional effects are present in seed implants with multiple sources. The different prostate compositions show $D_{90}$ differences of about 3\% while the effect of accounting for prostate density is a $2 \% D_{90}$ reduction. These two effects are of similar magnitude as the reported interseed effect, albeit smaller. Breast cases show a much higher sensitivity, partly because ${ }^{103} \mathrm{Pd}$ is more sensitive than ${ }^{125}$ I. Differences from TG-43 of $+30 \%$ are observed for mean A70/G30 breast meaning that clinical TG-43 $D_{w, w}$ calculations underestimate the dose to breast tissue. An additional $10 \%$ variation can be attributed to uncertainties in mammary gland and adipose tissue compositions while the effect of using the CT density is a $4 \%$ increase in $D_{90}$. If $A 80 / G 20$ breast is representative of the average breast cancer patient then our $A 70 / G 30$ breast results indicate that the compositional uncertainty translates into second order effects compared to the effect of going from water to average breast tissue. The use of breast density from CT data and the reported interseed effect are of third order. Nevertheless, density should be used in brachytherapy dose calculations given the accuracy with which it can be extracted from CT data.

The importance of dose differences reported in this work between tissue and water supports the use of accurate dose calculation algorithms such as MC for low energy brachytherapy. A detailed analysis of uncertainties in brachytherapy seed implants such as edema, seed migration, source strength calibration, seed positioning and modeling is required to place our findings in context.

Imaging techniques such as dual energy $\mathrm{CT}$ or spectral $\mathrm{CT}$, which can provide compositional information such as $Z_{\text {eff }}$, have the potential to diminish the influence of uncertainties on tissue compositions and may provide increased accuracy in low energy brachytherapy $\mathrm{MC}$ dosimetry.

\section{ACKNOWLEDGEMENTS}

This work is supported by grant \#017133 of the National Cancer Institute of Canada ( $\mathrm{NCIC})$. GL is supported by the Natural Sciences and Engineering Research Council of Canada (NSERC). BR is supported by a Marie Curie Reintegration Grant (grant agree- 
ment no. PIRG05-GA-2009-247878 from FP7-PEOPLE-2009-RG). The authors would like to acknowledge Michel D'Amours and Hossein Afsharpour for GEANT4 technical support. They would like to thank Dr Mark J. Rivard for useful discussions.

\section{REFERENCES}

${ }^{1}$ H.H. Holm and J. Gammelgaard, "Ultrasonically guided precise needle placement in the prostate and the seminal vesicles," J Urol 125, 385-387 (1981).

${ }^{2}$ H.H. Holm, N. Juul, J.F. Pedersen, H. Hansen, and I. Stroyer, "Transperineal 125iodine seed implantation in prostatic cancer guided by transrectal ultrasonography," J Urol 130, 283286 (1983).

${ }^{3}$ R.G. Stock, N.N. Stone, A. Tabert, C. lannuzzi, and J.K. DeWyngaert, "A dose-response study for I-125 prostate implants," Int J Radiat Oncol Biol Phys 41, 101-108 (1998).

${ }^{4}$ J.P. Pignol, B. Keller, E. Rakovitch, R. Sankreacha, H. Easton, and W. Que, "First report of a permanent breast 103Pd seed implant as adjuvant radiation treatment for early-stage breast cancer," Int J Radiat Oncol Biol Phys 64, 176-181 (2006).

${ }^{5}$ B. Keller, R. Sankreacha, E. Rakovitch, P. O'Brien, and J.P. Pignol, "A permanent breast seed implant as partial breast radiation therapy for early-stage patients: a comparison of palladium-103 and iodine-125 isotopes based on radiation safety considerations," Int J Radiat Oncol Biol Phys 62, 358-365 (2005).

${ }^{6}$ J.P. Pignol, E. Rakovitch, B.M. Keller, R. Sankreacha, and C. Chartier, "Tolerance and acceptance results of a palladium-103 permanent breast seed implant Phase I/II study," Int J Radiat Oncol Biol Phys 73, 1482-1488 (2009).

${ }^{7}$ R. Nath, L.L. Anderson, G. Luxton, K.A. Weaver, J.F. Williamson, and A.S. Meigooni, "Dosimetry of interstitial brachytherapy sources: recommendations of the AAPM Radiation Therapy Committee Task Group No. 43. American Association of Physicists in Medicine," Med Phys 22, 209-234 (1995).

${ }^{8}$ M.J. Rivard, et al., "Update of AAPM Task Group No. 43 Report: A revised AAPM protocol for brachytherapy dose calculations," Med Phys 31, 633-674 (2004).

${ }^{9}$ G.S. Burns and D.E. Raeside, "The accuracy of single-seed dose superposition for I-125 implants," Med Phys 16, 627-631 (1989).

${ }^{10} \mathrm{O}$. Chibani and J.F. Williamson, "MCPI: a sub-minute Monte Carlo dose calculation engine for prostate implants," Med Phys 32, 3688-3698 (2005).

${ }^{11}$ O. Chibani, J.F. Williamson, and D. Todor, "Dosimetric effects of seed anisotropy and interseed attenuation for 103Pd and 125 I prostate implants," Med Phys 32, 2557-2566 (2005).

${ }^{12}$ J.F. Carrier, L. Beaulieu, F. Therriault-Proulx, and R. Roy, "Impact of interseed attenuation and tissue composition for permanent prostate implants," Med Phys 33, 595-604 (2006).

${ }^{13}$ J.F. Carrier, M. D'Amours, F. Verhaegen, B. Reniers, A.G. Martin, E. Vigneault, and L. Beaulieu, "Postimplant dosimetry using a Monte Carlo dose calculation engine: a new clinical standard," Int J Radiat Oncol Biol Phys 68, 1190-1198 (2007).

${ }^{14}$ H. Afsharpour, M. D'Amours, B. Cote, J.F. Carrier, F. Verhaegen, and L. Beaulieu, "A Monte Carlo study on the effect of seed design on the interseed attenuation in permanent prostate implants," Med Phys 35, 3671-3681 (2008).

${ }^{15}$ C. Furstoss, et al., "Monte Carlo study of LDR seed dosimetry with an application in a clinical brachytherapy breast implant," Med Phys 36, 1848-1858 (2009).

${ }^{16}$ H. Afsharpour, J.P. Pignol, B. Keller, J.F. Carrier, B. Reniers, F. Verhaegen, and L. Beaulieu, "Influence of breast composition and interseed attenuation in dose calculations for post- 
implant assessment of permanent breast 103Pd seed implant," Physics in medicine and biology 55, 4547-4561 (2010).

${ }^{17}$ W. Kilby, J. Sage, and V. Rabett, "Tolerance levels for quality assurance of electron density values generated from CT in radiotherapy treatment planning," Phys Med Biol 47, 14851492 (2002).

${ }^{18}$ S.J. Thomas, "Relative electron density calibration of CT scanners for radiotherapy treatment planning," Br J Radiol 72, 781-786 (1999).

${ }^{19}$ U. Schneider, E. Pedroni, and A. Lomax, "The calibration of CT Hounsfield units for radiotherapy treatment planning," Phys Med Biol 41, 111-124 (1996).

${ }^{20}$ C. Constantinou, J.C. Harrington, and L.A. DeWerd, "An electron density calibration phantom for CT-based treatment planning computers," Med Phys 19, 325-327 (1992).

${ }^{21} \mathrm{H}$. Guan, F.F. Yin, and J.H. Kim, "Accuracy of inhomogeneity correction in photon radiotherapy from CT scans with different settings," Phys Med Biol 47, N223-231 (2002).

${ }^{22}$ W. Schneider, T. Bortfeld, and W. Schlegel, "Correlation between CT numbers and tissue parameters needed for Monte Carlo simulations of clinical dose distributions," Phys Med Biol 45, 459-478 (2000).

${ }^{23} \mathrm{~B}$. Vanderstraeten, et al., "Conversion of CT numbers into tissue parameters for Monte Carlo dose calculations: a multi-centre study," Phys Med Biol 52, 539-562 (2007).

${ }^{24}$ F.C. du Plessis, C.A. Willemse, M.G. Lotter, and L. Goedhals, "The indirect use of CT numbers to establish material properties needed for Monte Carlo calculation of dose distributions in patients," Med Phys 25, 1195-1201 (1998).

${ }^{25}$ N. Kanematsu, N. Matsufuji, R. Kohno, S. Minohara, and T. Kanai, "A CT calibration method based on the polybinary tissue model for radiotherapy treatment planning," Phys Med Biol 48, 1053-1064 (2003).

${ }^{26} \mathrm{~F}$. Verhaegen and S. Devic, "Sensitivity study for CT image use in Monte Carlo treatment planning," Phys Med Biol 50, 937-946 (2005).

${ }^{27}$ ICRP, Report of the task group on reference man. Report 23. 1975, Oxford: Pergamon.

${ }^{28}$ H.Q. Woodard and D.R. White, "The composition of body tissues," Br J Radiol 59, 1209-1218 (1986).

${ }^{29}$ ICRU, Tissue substitutes in radiation dosimetry and measurement. Report 44. 1989, Bethesda, MD.

${ }^{30}$ ICRU, Photon, electron, proton, and neutron interaction data for body tissues. Report 46. 1992, Bethesda, MD.

${ }^{31}$ R.L. Maughan, P.J. Chuba, A.T. Porter, E. Ben-Josef, and D.R. Lucas, "The elemental composition of tumors: kerma data for neutrons," Med Phys 24, 1241-1244 (1997).

${ }^{32}$ ICRP, Basic anatomical and physiological data for use in radiological protection: reference values. Report 89. 2002, Oxford: Pergamon.

${ }^{33}$ S. Agostinelli, et al., "Geant4-a Simulation Toolkit," Nuclear Instruments \& Methods in Physics Research Section a-Accelerators Spectrometers Detectors and Associated Equipment 506, 250-303 (2003).

${ }^{34}$ D. Cullen, J.H. Hubbell, and L. Kissel, "EPDL97: the Evaluated Photon Data Library, 97 version," UCRL-50400 Vol. 6, Rev 5, (1997).

${ }^{35}$ S.T. Perkins, D.E. Cullen, and S.M. Seltzer, "Tables and graphs of electron-interaction crosssections from $10 \mathrm{eV}$ to $100 \mathrm{GeV}$ derived from the LLNL Evaluated Electron Data Library (EEDL)," UCRL-50400 Vol. 31, (1997).

${ }^{36}$ S.T. Perkins, D.E. Cullen, M.H. Chen, J.H. Hubbell, J. Rathkopf, and J. Scofield, "Table and graphs of atomic subshell and relaxation data derived from the LLNL Evaluated Atomic Data Library (EADL)," UCRL-50400 Vol. 30, (1997). 
${ }^{37}$ J.V. Siebers, P.J. Keall, A.E. Nahum, and R. Mohan, "Converting absorbed dose to medium to absorbed dose to water for Monte Carlo based photon beam dose calculations," Phys Med Biol 45, 983-995 (2000).

${ }^{38} \mathrm{H}$. Liu, P. Keall, and W. Hendee, "Dm rather than Dw should be used in Monte Carlo treatment planning. ," Med Phys 29, 923-924 (2002).

${ }^{39}$ I.J. Chetty, et al., "Report of the AAPM Task Group No. 105: Issues associated with clinical implementation of Monte Carlo-based photon and electron external beam treatment planning," Med Phys 34, 4818-4853 (2007).

${ }^{40}$ I. Kawrakow, "Accurate condensed history Monte Carlo simulation of electron transport. I. EGSnrc, the new EGS4 version," Med Phys 27, 485-498 (2000).

${ }^{41}$ J.F. Williamson, "Monte Carlo evaluation of kerma at a point for photon transport problems," Med Phys 14, 567-576 (1987).

${ }^{42}$ E. Poon, J.F. Williamson, T. Vuong, and F. Verhaegen, "Patient-specific Monte Carlo dose calculations for high-dose-rate endorectal brachytherapy with shielded intracavitary applicator," Int J Radiat Oncol Biol Phys 72, 1259-1266 (2008).

${ }^{43}$ E. Poon and et al., "BrachyGUI: an adjunct to an accelerated Monte Carlo photon transport code for patient-specific brachytherapy dose calculations and analysis," Journal of Physics: Conference Series 102, 012018 (2008).

${ }^{44}$ H.E. Johns and J.R. Cunningham, The physics of radiology. 4th ed. 1983, Springfield, III., U.S.A.: Charles C. Thomas. xix, $796 \mathrm{p}$.

${ }^{45}$ R.D. Rosenberg, C.A. Kelsey, M.R. Williamson, J.D. Houston, and W.C. Hunt, "Computer-based collection of mammographic exposure data for quality assurance and dosimetry," Med Phys 28, 1546-1551 (2001).

${ }^{46}$ D.R. Dance, C.L. Skinner, and G.A. Carlsson, "Breast dosimetry," Appl Radiat Isot 50, 185-203 (1999).

${ }^{47}$ I. Kawrakow, E. Mainegra-Hing, F. Tessier, and B.R.B. Walters, The EGSnrc C++ class library, NRC Report PIRS-898 (rev A). 2009: Ottawa, Canada.

${ }^{48}$ F. Salvat, J.M. Fernandez-Varea, E. Acosta, and J. Sempau, PENELOPE-A code system for Monte Carlo simulations of electron and photon transport. 2001, NEA-OECD: Paris.

${ }^{49}$ J.F. Almansa, R. Guerrero, F.M.O. Al-Dweri, M. Anguiano, and A.M. Lallena, "Dose distribution in water for monoenergetic photon point sources in the energy range of interest in brachytherapy: Monte Carlo simulations with PENELOPE and GEANT4," Radiation Physics and Chemistry 76, 766-773 (2007).

${ }^{50}$ R.E. Taylor and D.W. Rogers, "An EGSnrc Monte Carlo-calculated database of TG-43 parameters," Med Phys 35, 4228-4241 (2008).

${ }^{51}$ M.J. Rivard, "Brachytherapy dosimetry parameters calculated for a 131Cs source," Med Phys 34, 754-762 (2007).

${ }^{52}$ D. Liu, E. Poon, M. Bazalova, B. Reniers, M. Evans, T. Rusch, and F. Verhaegen, "Spectroscopic characterization of a novel electronic brachytherapy system," Phys Med Biol 53, 61-75 (2008).

${ }^{53}$ B.R. Walters, I. Kawrakow, and D.W. Rogers, "History by history statistical estimators in the BEAM code system," Med Phys 29, 2745-2752 (2002).

${ }^{54}$ J. Perez-Calatayud, D. Granero, and F. Ballester, "Phantom size in brachytherapy source dosimetric studies," Med Phys 31, 2075-2081 (2004).

${ }^{55}$ J.H. Hubbell and S.M. Seltzer, Tables of X-Ray Mass Attenuation Coefficients and Mass EnergyAbsorption Coefficients. 1996, NIST.

${ }^{56}$ M.J. Yaffe, et al., "The myth of the 50-50 breast," Med Phys 36, 5437-5443 (2009). 


\section{CHAPTER}

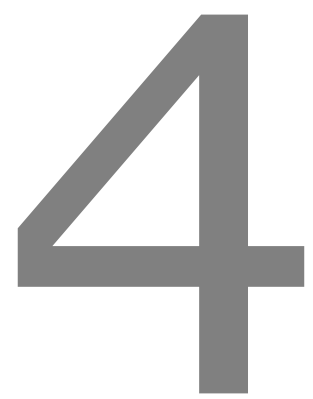

The difference of scoring dose to water or tissues in Monte Carlo dose calculations for low energy brachytherapy photon sources

Guillaume Landry, Brigitte Reniers, Jean-Philippe Pignol, Luc Beaulieu, Frank Verhaegen

Published in: Medical Physics 38(3):1526-1533, 2011. 


\section{ABSTRACT}

Purpose The goal of this work is to compare $D_{\mathrm{m}, \mathrm{m}}$ (radiation transported in medium, dose scored in medium) and $D_{\mathrm{w}, \mathrm{m}}$ (radiation transported in medium, dose scored in water) obtained from Monte Carlo (MC) simulations for a subset of human tissues of interest in low energy photon brachytherapy. Using low dose rate (LDR) seeds and an electronic brachytherapy source (EBS), we quantify the large cavity theory conversion factors required. We also assess whether applying large cavity theory utilizing the sources' initial photon spectra and average photon energy induces errors related to spatial spectral variations. First, ideal spherical geometries were investigated followed by clinical brachytherapy LDR seed implants for breast and prostate cancer patients.

Methods Two types of dose calculations are performed with the GEANT4 MC code. (1) For several human tissues, dose profiles are obtained in spherical geometries centered on four types of low energy brachytherapy sources: ${ }^{125} \mathrm{I},{ }^{103} \mathrm{Pd}$ and ${ }^{131} \mathrm{Cs}$ seeds as well as an EBS operating at $50 \mathrm{kV}$. Ratios of $D_{\mathrm{w}, \mathrm{m}}$ over $D_{\mathrm{m}, \mathrm{m}}$ are evaluated in the $0-6 \mathrm{~cm}$ range. In addition to mean tissue composition, compositions corresponding to one standard deviation from the mean are also studied. (2) Four clinical breast (using ${ }^{103} \mathrm{Pd}$ ) and prostate (using ${ }^{125} \mathrm{I}$ ) brachytherapy seed implants are considered. MC dose calculations are performed based on post-implant CT scans using prostate and breast tissue compositions. PTV $D_{90}$ values are compared for $D_{\mathrm{w}, \mathrm{m}}$ and $D_{\mathrm{m}, \mathrm{m}}$.

Results (1) Differences $\left(D_{\mathrm{w}, \mathrm{m}} / D_{\mathrm{m}, \mathrm{m}}-1\right)$ of $-3 \%$ to $70 \%$ are observed for the investigated tissues. For a given tissue, $D_{\mathrm{w}, \mathrm{m}} / D_{\mathrm{m}, \mathrm{m}}$ is similar for all sources within $4 \%$ and does not vary more than $2 \%$ with distance, due to very moderate spectral shifts. Variations of tissue composition about the assumed mean composition influence the conversion factors up to $38 \%$. (2) The ratio of $D_{90(w, m)}$ over $D_{90(m, m)}$ for clinical implants matches $D_{\mathrm{w}, \mathrm{m}} / D_{\mathrm{m}, \mathrm{m}}$ at $1 \mathrm{~cm}$ from the single point sources.

Conclusion Given the small variation with distance, using conversion factors based on the emitted photon spectrum (or its mean energy) of a given source introduces minimal error. The large differences observed between scoring schemes underline the need for guidelines on choice of media for dose reporting. Providing such guidelines is beyond the scope of this work. 


\section{INTRODUCTION}

The dosimetry of low energy photon (from a few to about $50 \mathrm{keV}$ ) brachytherapy procedures based on low dose rate (LDR) seed implants using ${ }^{125} \mathrm{I}$ and ${ }^{103} \mathrm{Pd}$ or high dose rate (HDR) electronic brachytherapy sources (EBS) follows the AAPM Radiation Therapy Committee Task Group 43 formalism ${ }^{1,2}$ (TG-43). The use of dosimetric functions obtained in uniform water spheres of $15 \mathrm{~cm}$ radius has reported shortcomings, such as the inability to handle tissue heterogeneities and interseed attenuation in prostate and breast seed implants ${ }^{3-6}$. A study on eye plaque dosimetry ${ }^{7}$ has shown similar shortcomings.

Model based dose calculation (MBDC) algorithms such as Monte Carlo (MC) simulations, collapsed cone convolution and Boltzmann transport equation solvers take into account relevant radiation physics; they are all considered by the active AAPM Radiation Therapy Committee Task Group 186 (TG-186) as potential replacements to the TG-43 formalism. MBDC algorithms enable photon transport in arbitrary media for calculation of the absorbed dose $D_{\mathrm{m}, \mathrm{m}}$ to a small mass of medium $\mathrm{m}$ embedded in the surrounding transport medium $m$, or the absorbed dose $D_{\mathrm{w}, \mathrm{m}}$ to a small mass of water $w$ in the surrounding medium. These two scoring quantities as well as $D_{\mathrm{w}, \mathrm{w}}$, the scoring quantity recommended by TG-43, are schematically compared in Figure 4.1. When considering $D_{\mathrm{w}, \mathrm{m}}$, the small mass of water is only involved during the scoring of the energy deposited, but not during the transport of photons. In other words, scoring absorbed dose to water does not perturb the photon fluence in the surrounding medium.

(a) $D_{\mathrm{m}, \mathrm{m}}$

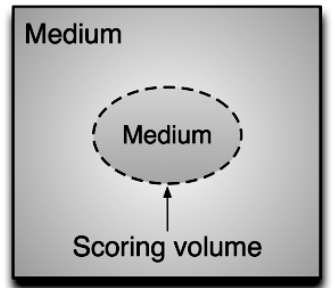

(b) $D_{\mathrm{w}, \mathrm{m}}$

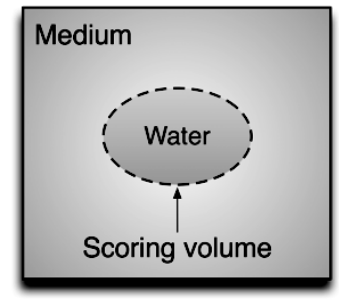

(c) $D_{\mathrm{w}, \mathrm{w}}$

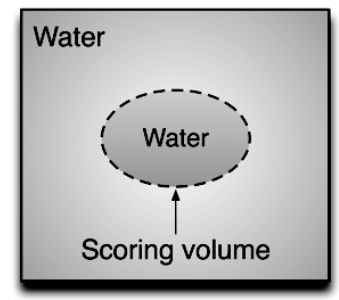

Figure 4.1: (a) Dose to medium with transport in medium, $D_{m, m}$, where the scoring volume is filled with the transport medium. (b) Dose to water with transport in medium, $D_{w, m}$ where the scoring volume is filled with water. The water is only considered for scoring purposes and does not affect the transport. (c) Dose to water with transport in water, $D_{w, w}$ representing the TG-43 dose calculation conditions. Scales are arbitrary.

In external beam radiotherapy (EBRT), where treatment planning based on MBDC simulations has already been adopted, the AAPM Radiation Therapy Committee Task Group $105^{8}$ (TG-105) guidelines do not favor one scoring quantity over the other. The difference between the two quantities has been reported to be small for tissues other than bone ${ }^{9,10}$. Special treatment has been given to bone (spongiosa) since radiosensitive tissues such as red bone marrow and bone surface cells are imbedded in a matrix 
of high density trabecular bone ${ }^{10}$. It was found that $D_{\mathrm{w}, \mathrm{m}}$ is closer to the dose to sensitive tissues in spongiosa. For tissues in general, arguments for both quantities have been presented in $\mathrm{EBRT}^{9,11}$ and the ICRU has recently issued a recommendation favoring the use of $D_{\mathrm{w}, \mathrm{m}}{ }^{12}$.

In low energy brachytherapy, cavity theory ${ }^{13}$ for secondary electron ranges shorter than the cavity size, which is commonly known as large cavity theory, can be used to convert $D_{\mathrm{m}, \mathrm{m}}$ to $D_{\mathrm{w}, \mathrm{m}}$. In this work, a cavity is considered to be a voxel with a size of the order of $1 \mathrm{~mm}$ and the range of secondary electrons is at most $43 \mu \mathrm{m}$ (in the continuous slowing down approximation for a $50 \mathrm{keV}$ electron in water). Under the assumptions of this theory, $D_{\mathrm{w}, \mathrm{m}}$ is given by:

$$
D_{\mathrm{w}, \mathrm{m}}=D_{\mathrm{m}, \mathrm{m}} \frac{\left(\bar{\mu}_{\mathrm{en}} / \rho\right)_{\mathrm{w}}}{\left(\bar{\mu}_{\mathrm{en}} / \rho\right)_{\mathrm{m}}}=D_{\mathrm{m}, \mathrm{m}}\left(\bar{\mu}_{\mathrm{en}} / \rho\right)_{\mathrm{m}}^{\mathrm{w}}
$$

where $\left(\bar{\mu}_{\text {en }} / \rho\right)_{\mathrm{w}}$ and $\left(\bar{\mu}_{\text {en }} / \rho\right)_{\mathrm{m}}$ are the mass energy absorption coefficients of water and medium averaged over the local photon energy fluence.

Since the photoelectric effect plays a major role in the low energy photon range and given its strong dependence on elemental composition, significant differences between the mass energy absorption coefficients of human tissues and water are observed. This induces differences in $D_{\mathrm{m}, \mathrm{m}}$ and $D_{\mathrm{w}, \mathrm{m}}$ which are larger than in EBRT or even in ${ }^{192}$ Ir HDR brachytherapy ${ }^{14}$. The amplitude of these differences has been studied in phantom materials for ${ }^{125}$ I sources ${ }^{15,16}$ where $\left(\bar{\mu}_{\text {en }} / \rho\right)_{\mathrm{m}}^{\mathrm{w}}$ varied from 0.91 for solid water to 2.7 for polystyrene. Rivard et al. ${ }^{14}$ studied the differences for bone and breast tissue using mono-energetic point sources, finding $D_{\mathrm{m}, \mathrm{m}}$ 5-7 times higher than $D_{\mathrm{w}, \mathrm{m}}$ for bone. Because these differences are much larger than in EBRT they recommend the reporting of $D_{\mathrm{m}, \mathrm{m}}$ along with $D_{\mathrm{w}, \mathrm{w}}$.

The goal of this study is to compare $D_{\mathrm{m}, \mathrm{m}}$ and $D_{\mathrm{w}, \mathrm{m}}$ obtained from MC simulations for a subset of human tissues of interest in brachytherapy. Using LDR seeds and an EBS, we quantify the large cavity theory conversion factors required. We also assess whether applying large cavity theory, utilizing the sources' initial photon spectra and average photon energy, introduces errors related to spatial spectral variations. The first part of the study investigates ideal spherical geometries while the second part investigates clinical brachytherapy LDR seed implants for breast and prostate cancer patients.

\section{MATERIALS AND METHODS}

\section{Monte Carlo code and dose analysis tool}

Version 9.3 of the general purpose MC simulation toolkit GEANT4 ${ }^{17}$ was used for this work. The photoelectric effect, Compton scattering and Rayleigh scattering 
processes of the Lawrence Livermore National Laboratory low energy electromagnetic model were activated. This model uses the $\mathrm{EPDL}^{18}, \mathrm{EEDL}^{19}$ and $\mathrm{EADL}^{20}$ evaluated data libraries. Since the range of secondary electrons is very short compared to voxel size for the low photon energies of interest, dose was approximated by collision kerma, i.e. electrons were not tracked. The EEDL library was necessary for the simulation of photon production inside the EBS.

Photon transport is not influenced by the scoring medium and is always simulated in the local medium. $D_{\mathrm{m}, \mathrm{m}}$ was scored using an analog estimator while $D_{\mathrm{w}, \mathrm{m}}$ was scored using a track length estimator ${ }^{21}$. When using track length estimation the $D_{w, m}^{j}$ (approximated by the collision kerma to water in medium, $K_{w, m}^{j}$ ) deposited in a scoring region of arbitrary shape by a photon $j$ is given by:

$$
D_{\mathrm{w}, \mathrm{m}}^{j}=K_{\mathrm{w}, \mathrm{m}}^{j}=\frac{E_{j}\left(\mu_{\mathrm{en}} / \rho\right)_{\mathrm{w}} l_{j}}{V},
$$

where $E_{j}$ is the photon's energy, $l_{j}$ is the straight line distance travelled inside the scoring region of volume $V$ and $\left(\mu_{\mathrm{en}} / \rho\right)_{\mathrm{w}}$ is the mass energy absorption coefficient of water at $E_{j}$. While track length scoring of $D_{\mathrm{m}, \mathrm{m}}$ would be more efficient, we chose analog scoring to avoid potential systematic errors from tabulating $\left(\mu_{\mathrm{en}} / \rho\right)_{\mathrm{m}}$ for a large number of materials in the GEANT4 application and because calculation times were not an issue for this work. Mass energy absorption coefficients $\left(\mu_{\mathrm{en}} / \rho\right)_{\mathrm{w}}$ of water were calculated at $1 \mathrm{keV}$ intervals using the EGSnrc ${ }^{22}$ (version V4-r2-3-1) user code g. Values of $\left(\mu_{\mathrm{en}} / \rho\right)_{\mathrm{m}}$ were also calculated with $\mathrm{g}$ for the tissues of interest in this study for comparison with our simulation results. The EGSnrc code was chosen after verifying that the EPDL (used by GEANT4) and $\mathrm{XCOM}^{23}$ (used by EGSnrc) cross section libraries were equivalent.

BrachyGUI ${ }^{24}$ is a brachytherapy dose analysis and planning tool which was used in this work to process DICOM CT imaging data, to create input files for GEANT4 patient simulations and to analyze dose distributions.

\section{Monte Carlo simulations in uniform geometries}

Four brachytherapy sources were investigated in this study: model $2301^{125}$ I source and model $2335{ }^{103} \mathrm{Pd}$ source (Best Medical, Springfield, VA), model Cs-1Rev2 ${ }^{131}$ Cs source (IsoRay Medical, Richland, WA) and model S700 Axxent EBS (Xoft, Sunnyvale, CA) source. The ${ }^{125} \mathrm{I},{ }^{103} \mathrm{Pd}$ and ${ }^{131} \mathrm{Cs}$ sources were modeled in GEANT4 according to published descriptions ${ }^{25,26}$. The agreement of source modeling with published descriptions has been reported in our previous work ${ }^{27}$. The Axxent source operating at $50 \mathrm{kV}$ placed inside its cooling water sheath was simulated with a GEANT4 model developed by Liu et al. ${ }^{28}$

Phase space files containing the photon distribution were collected at the outer surface of each source and photon spectra accounting for the spectral shift caused by the encapsulation were extracted from them. The spectra were collected over the whole phase space file, averaging out the source anisotropy. A $5 \mathrm{keV}$ lower energy 
cutoff was applied, neglecting the $4.5 \mathrm{keV}$ characteristic x-rays produced in the titanium encapsulation, which only affects the dose in the first $\mathrm{mm}$ from the source ${ }^{29}$.

The mean photon energies for model $2335\left({ }^{103} \mathrm{Pd}\right)$, model $2301\left({ }^{125} \mathrm{I}\right)$, model Cs1 Rev2 $\left({ }^{131} \mathrm{Cs}\right)$ and $50 \mathrm{kV}$ Axxent emission spectra (lower energy cutoff applied) are 21.0 $\mathrm{keV}, 28.5 \mathrm{keV}, 30.4 \mathrm{keV}$ and $26.9 \mathrm{keV}$, respectively.

Each of these spectra was used to emit photons from the point source located at the center of a $100 \mathrm{~cm}$ sphere of uniform composition, ignoring anisotropy. Such a large radius was chosen to simulate an infinite geometry and provide full backscatter. Dose per emitted photon was scored for the first $10 \mathrm{~cm}$ in $1 \mathrm{~mm}$ thick spherical shells whose mid radii were centered at every millimeter for both the local medium $\left(D_{\mathrm{m}, \mathrm{m}}\right)$ and water $\left(D_{\mathrm{w}, \mathrm{m}}\right)$. The tissues under investigation are the same as in our previous work $^{27}$ with the addition of Muscle and Skin whose compositions are from Woodard and White ${ }^{30}$. The elemental composition of each tissue as well as its mass density and effective atomic number $Z_{\text {eff }}$ are tabulated in the online supplemental data accompanying this publication.

For most tissue types the mean $(M)$ composition as well as compositions removed by one standard deviation $(M-\sigma, M+\sigma)$ from the mean, as defined in Woodard and White ${ }^{30}$, are used and are indexed as $h i-Z$, mean- $Z$ and $l o-Z$ according to their $Z_{\text {eff }}$. Distributions of radial dose per emitted photon are obtained for each tissue by simulating $10^{8}$ photon histories for $D_{\mathrm{w}, \mathrm{m}}$ and $2 \cdot 10^{9}$ for $D_{\mathrm{m}, \mathrm{m}}$, ensuring statistical uncertainty (calculated according to Walters et al. ${ }^{31}$ ) less than $0.5 \%$ at $10 \mathrm{~cm}$. The higher number of histories for $D_{\mathrm{m}, \mathrm{m}}$ is required given analog scoring's lower efficiency.

Breast tissue (Breast $A 70$ G30) was modeled as a uniform mixture with a mass fraction of $70 \%$ Adipose mean-Z and $30 \%$ Mammary gland mean- $Z$. The mass fraction of each component was chosen according to Yaffe et al. ${ }^{32}$ who report a mean mammary gland volumetric percentage of $20 \%$ with $80 \%$ of women having gland volumetric percentage below $27 \%$.

\section{Clinical cases}

\section{Prostate cancer patients}

MC dose calculations were performed for four prostate cancer patients who underwent transperineal implantation using model $2301^{125}$ I sources under transrectal ultrasound guidance at the MAASTRO Clinic where a 145 Gy prescription dose is given to the ultrasound prostate contour, taken as the planning target volume (PTV). Patient description is presented in Landry et al. ${ }^{27} \mathrm{CT}$ scans with $3 \mathrm{~mm}$ slice thickness taken within 24 hrs of implantation were imported in the VariSeed planning system (Varian, Palo Alto, CA) where prostate contours were drawn by a radiation oncologist and seed positions established using VariSeed's seed finder algorithm. 


\section{Breast cancer patients}

Dose calculations were also performed for four breast cancer patients from the Sunnybrook Health Sciences Centre (Toronto, Canada) who underwent permanent breast seed implants ${ }^{33}$ using the model $2335{ }^{103} \mathrm{Pd}$ seed. Patient description is presented in Landry et al. $^{27}$ A 90 Gy dose was prescribed, covering the PTV which consists of "the lumpectomy cavity plus a margin of $1 \mathrm{~cm}$ modified to $5 \mathrm{~mm}$ deep to the skin surface and also along the fascia pectoralis" ${ }^{\prime 33}$. CT scans with $5 \mathrm{~mm}$ slice thickness taken 2 months post implantation with PTV contours drawn by a radiation oncologist and seed positions were exported from VariSeed.

Dose calculations

DICOM data were used to create material compositions per voxel and density assignments for both types of patients. The mass density of each voxel was established according to the CT scanner's HU - $\rho$ calibration curve. Voxels within PTV contours were assigned prostate tissue (Prostate $A, B$ and $C$ ) or breast tissue (Breast A70G30) compositions while those outside had the composition of water. Assigning water outside is not an issue when considering doses inside the PTV only, but will affect doses to organs at risk, which are not of interest for this paper. GEANT4 simulations were performed for each patient as described in our previous work ${ }^{27}$ using phase space files as photon sources, effectively ignoring the interseed effect ${ }^{6}$, which is not under investigation here. $D_{\mathrm{m}, \mathrm{m}}$ and $D_{\mathrm{w}, \mathrm{m}}$ were scored for $5 \cdot 10^{8}$ and $5 \cdot 10^{7}$ photon histories respectively. The dose calculation grid was $2 \mathrm{~mm} \times 2 \mathrm{~mm} \times 3 \mathrm{~mm}(X \times Y \times$ slice thickness) for prostate patients and $1 \mathrm{~mm} \times 1 \mathrm{~mm} \times 5 \mathrm{~mm}$ for breast patients.

\section{RESULTS}

\section{Idealized geometries}

Figures 4.2(a) shows radial dose profiles in Adipose mean-Z for each source in units of dose (Gy) per emitted particle. The inverse square law dependence associated with the point source geometry has been factored out by multiplying dose values by $r^{2}$, where $r$ is the radial distance from the source's center to the scoring shell's mid-radius. The ${ }^{131} \mathrm{Cs}$ results, being quite similar to ${ }^{125} \mathrm{I}$ due to their similar photon emission spectra, have been omitted. In addition to dose profiles, Figure 4.2(b) shows the ratio $D_{\mathrm{w}, \mathrm{m}} / D_{\mathrm{m}, \mathrm{m}}$ which corresponds to the factor required to convert one type of dose reporting to the other under the large cavity assumption. 

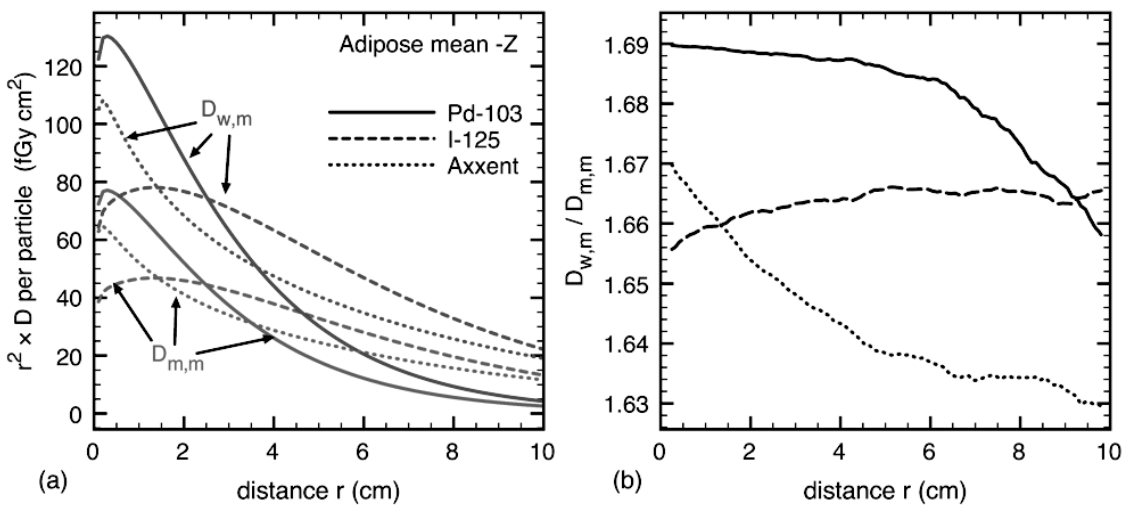

Figure 4.2: (a) Radial $D_{w, m}$ and $D_{m, m}$ in Adipose mean-Z corrected for the inverse square law for three different brachytherapy photon sources. (b) Ratio $D_{w, m} / D_{m, m}$ illustrating spatial variation in conversion factor. $D_{w, m} / D_{m, m}$ values have been smoothed using least square smoothing with four neighboring points to reduce statistical noise.

Figure 4.2 clearly demonstrates that there are important differences between $D_{\mathrm{w}, \mathrm{m}}$ and $D_{\mathrm{m}, \mathrm{m}}$ for all sources; $D_{\mathrm{w}, \mathrm{m}}$ is almost $70 \%$ higher for Adipose mean-Z. Interestingly, $D_{\mathrm{w}, \mathrm{m}} / D_{\mathrm{m}, \mathrm{m}}$ values are similar within $4 \%$ for the three sources. Figure 4.3 shows the $\mu_{\mathrm{en}} / \rho$ ratio Water to Adipose mean- $Z$ as a function of photon energy where in the 10-30 keV range, the $\mu_{\mathrm{en}} / \rho$ ratio reaches its maximum and has a relatively stable behavior, which explains the similar $D_{\mathrm{w}, \mathrm{m}} / D_{\mathrm{m}, \mathrm{m}}$ values between sources.

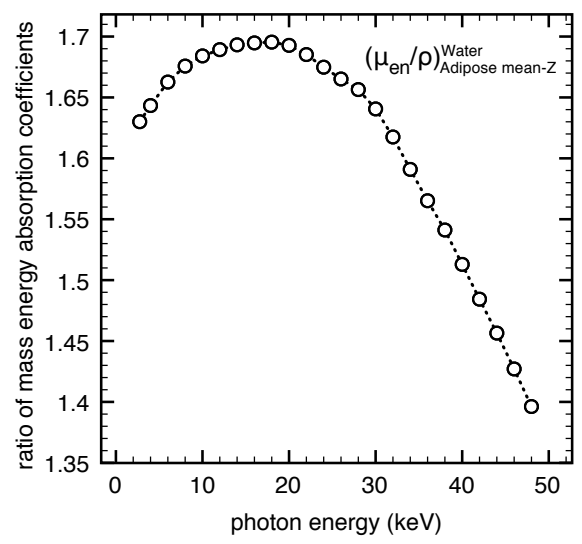

Figure 4.3: Variation of the ratio of mass energy absorption coefficients, Water to Adipose mean-Z, with photon energy. Data from calculations with EGSnrc user code $g$.

Figure 4.2 (b) shows that variation with distance of $D_{\mathrm{w}, \mathrm{m}} / D_{\mathrm{m}, \mathrm{m}}$ is at most $3 \%$ over $10 \mathrm{~cm}$ for the Axxent and ${ }^{103} \mathrm{Pd}$ sources while it is less than $1 \%$ for ${ }^{125}$ I. We note that $D_{\mathrm{w}, \mathrm{m}} / D_{\mathrm{m}, \mathrm{m}}$ increases slightly with distance for ${ }^{125} \mathrm{I}$ as opposed to the other sources. 
Figure 4.4 shows the mean photon energy variation with distance for each source where we see that the Axxent and ${ }^{103} \mathrm{Pd}$ spectra undergo hardening with depth while the ${ }^{125}$ I spectrum softens slightly due to a buildup of scattered photons. This is due to the narrow range of energies present in the ${ }^{125}$ I spectrum (from $27.2 \mathrm{keV}$ to $35.5 \mathrm{keV}$ ), which limits its sensitivity to spectral shifts. The Axxent source has a soft continuous spectrum ${ }^{28}$ and undergoes significant hardening in the first $4 \mathrm{~cm}$.

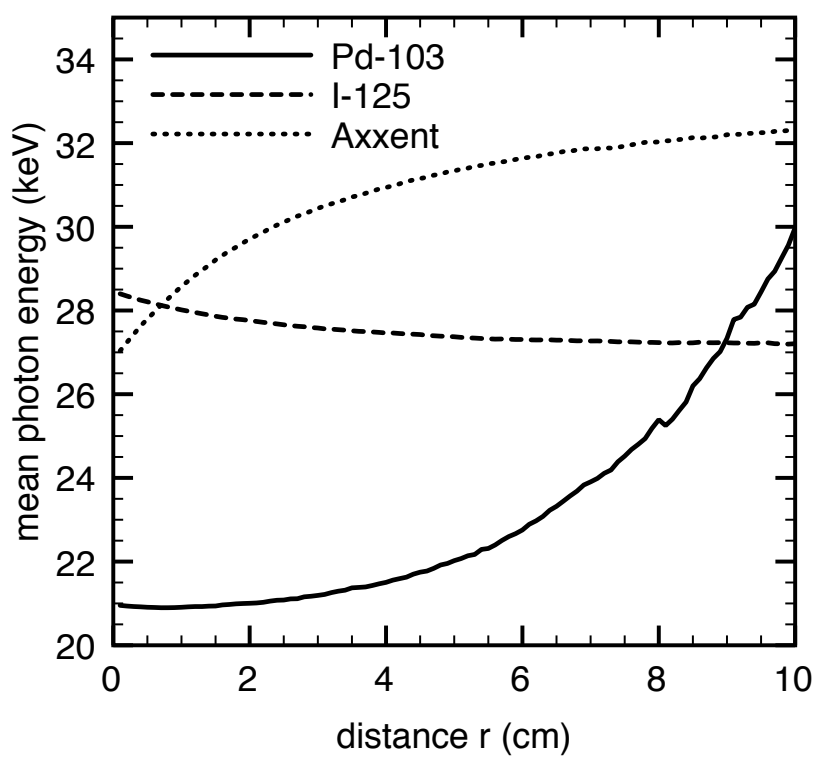

Figure 4.4: Variation of mean photon energy from three brachytherapy sources with distance in adipose tissue.

Although Adipose mean- $Z$ has the second lowest $Z_{\text {eff }}$ of the investigated tissues (14\% lower than Water) the trends described above apply also to the other tissues of interest.

Figure 4.5 shows $D_{\mathrm{w}, \mathrm{m}}$ compared to $D_{\mathrm{m}, \mathrm{m}}$ for Breast $A 70 \mathrm{G} 30$ and Prostate $C$. We see that for breast tissue, large (50\%) differences are found between the two scoring methods. For the Prostate $C$ composition, the differences are less striking but still important (8\%). 

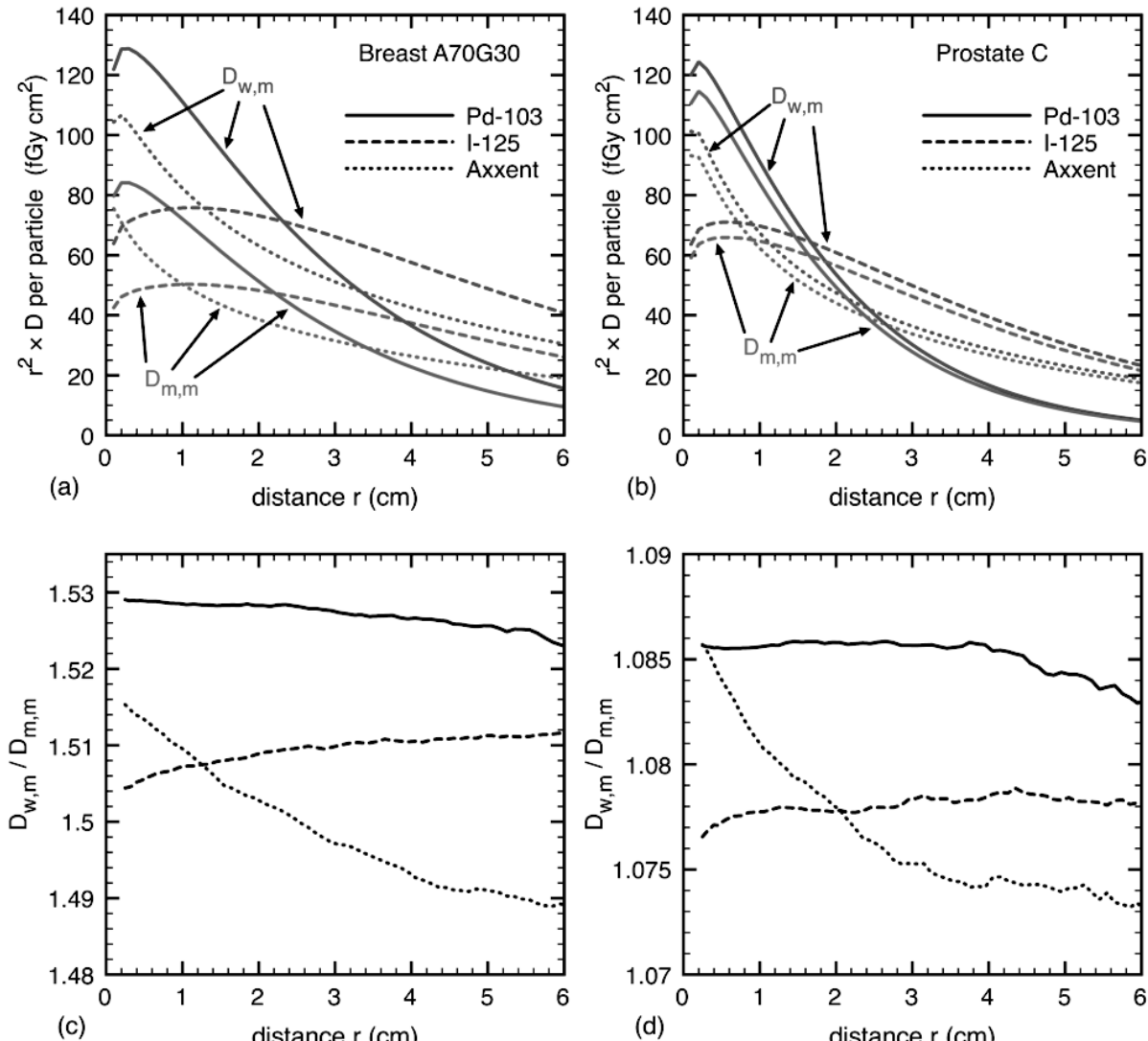

(c)

distance $r(\mathrm{~cm})$

(d)

distance $r(\mathrm{~cm})$

Figure 4.5: (a) Radial $D_{w, m}$ and $D_{m, m}$ in Breast A70G30 corrected for the inverse square law for three different brachytherapy photon sources. (b) Radial $D_{w, m}$ and $D_{m, m}$ in Prostate C. (c) Ratio $D_{w, m} / D_{m, m}$ illustrating spatial variation in conversion factor for Breast A70G30. (d) Ratio for Prostate $C$.

The results of simulations in all tissues are presented in Table 4.1 where values of $D_{\mathrm{w}, \mathrm{m}} / D_{\mathrm{m}, \mathrm{m}}$ are reported. The $\mu_{\mathrm{en}} / \rho$ ratio (from the EGSnrc user code g) at the initial mean photon energy $\left\langle E_{0}\right\rangle,\left(\mu_{\text {en }} / \rho\right)_{\mathrm{m},\left\langle E_{0}\right\rangle}^{\mathrm{w}}$, is also given. For clarity, only mean-Z compositions are presented in Table 4.1. The $\left(\mu_{\mathrm{en}} / \rho\right)_{\mathrm{m}, \Psi}^{\mathrm{w}}$ ratio derived from Equation 4.1, using the energy fluence $\Psi$ from the initial photon spectrum agreed with $\left(\mu_{\text {en }} / \rho\right)_{\mathrm{m},\left\langle E_{0}\right\rangle}^{\mathrm{w}}$ within $1 \%$ for isotope sources and within $2 \%$ for the EBS source. 
Table 4.1: $D_{w, m} / D_{m, m}$ for mean-Z tissue compositions at $1 \mathrm{~cm}$ distance from the source as well as the minimum and maximum values observed in the $0.1-6 \mathrm{~cm}$ range for ${ }^{125} \mathrm{l},{ }^{103} \mathrm{Pd}$ and the Axxent source. $D_{w, m} / D_{m, m}$ values are compared to ratios of mass energy absorption coefficients at the mean energy of the initial photon spectrum obtained from the EGSnrc user code $g$.

\begin{tabular}{ccccc}
\hline \hline Tissue & Source & $\begin{array}{c}D_{\mathrm{w}, \mathrm{m}} / D_{\mathrm{m}, \mathrm{m}} \\
(r=1 \mathrm{~cm})\end{array}$ & $\begin{array}{c}D_{\mathrm{w}, \mathrm{m}} / D_{\mathrm{m}, \mathrm{m}} \\
{[\mathrm{min}-\mathrm{max}]}\end{array}$ & $(\mu / \rho)_{\mathrm{m},\left\langle E_{0}\right\rangle}^{\mathrm{w}}$ \\
\hline Adipose & ${ }^{125} \mathrm{I}$ & 1.66 & $1.65-1.67$ & 1.65 \\
& ${ }^{103} \mathrm{Pd}$ & 1.69 & $1.68-1.69$ & 1.70 \\
& Axxent & 1.66 & $1.64-1.67$ & 1.66 \\
Mammary gland & ${ }^{125} \mathrm{I}$ & 1.24 & $1.24-1.24$ & 1.24 \\
& ${ }^{103} \mathrm{Pd}$ & 1.25 & $1.25-1.25$ & 1.25 \\
& Axxent & 1.24 & $1.23-1.25$ & 1.24 \\
Muscle & ${ }^{125} \mathrm{I}$ & 0.97 & $0.97-0.97$ & 0.97 \\
& ${ }^{103} \mathrm{Pd}$ & 0.98 & $0.98-0.98$ & 0.98 \\
& Axent & 0.97 & $0.97-0.98$ & 0.97 \\
Prostate A & ${ }^{225} \mathrm{I}$ & 1.00 & $1.00-1.00$ & 0.99 \\
& ${ }^{103} \mathrm{Pd}$ & 1.00 & $1.00-1.00$ & 1.00 \\
& Axent & 1.00 & $1.00-1.00$ & 1.00 \\
Prostate C & ${ }^{125} \mathrm{I}$ & 1.08 & $1.08-1.08$ & 1.07 \\
& ${ }^{103} \mathrm{Pd}$ & 1.09 & $1.08-1.09$ & 1.08 \\
& Axxent & 1.08 & $1.07-1.09$ & 1.07 \\
& ${ }^{125} \mathrm{I}$ & 1.06 & $1.06-1.06$ & 1.06 \\
& ${ }^{103} \mathrm{Pd}$ & 1.07 & $1.07-1.07$ & 1.07 \\
& Axxent & 1.07 & $1.06-1.07$ & 1.06 \\
Breast A70G30 & ${ }^{125} \mathrm{I}$ & 1.51 & $1.50-1.51$ & 1.53 \\
& ${ }^{103} \mathrm{Pd}$ & 1.53 & $1.52-1.53$ & 1.51 \\
\hline \hline
\end{tabular}

From Table 4.1 we see that tissues with large $Z_{\text {eff }}$ differences from water such as Adipose and Mammary gland have important differences between $D_{\mathrm{w}, \mathrm{m}}$ and $D_{\mathrm{m}, \mathrm{m}}$. This is expected given the large difference between mass energy absorption coefficients of these tissues and water. On the other hand, tissues such as Muscle and Prostate $A$ appear to be more water equivalent and less affected by the choice of scoring medium, although differences of $3 \%$ are observed for Muscle. Prostate $B$ was found to be equivalent to Prostate $A$ and is not shown while Prostate $C$ exhibits an $8-9 \%$ higher $D_{\mathrm{w}, \mathrm{m}}$ at $1 \mathrm{~cm}$. Muscle is the only tissue whose composition has a higher $Z_{\text {eff }}$ than Water. For this reason its $D_{\mathrm{w}, \mathrm{m}} / D_{\mathrm{m}, \mathrm{m}}$ values are all smaller than unity. We observe important differences for Breast A70G30 given the important proportion of adipose tissue present in breast.

For any given tissue and source, the variation of $D_{\mathrm{w}, \mathrm{m}} / D_{\mathrm{m}, \mathrm{m}}$ with distance from the source is less than $2 \%$ in the $0-6 \mathrm{~cm}$ range. Using the emission photon spectrum or its mean energy instead of the real local spectrum to calculate conversion factors based on $\mu_{\mathrm{en}} / \rho$ ratio water to tissue will only introduce a small $(<2 \%)$ error.

Table 4.2 provides the $D_{\mathrm{w}, \mathrm{m}} / D_{\mathrm{m}, \mathrm{m}}$ values at $1 \mathrm{~cm}$ for $h i-Z$, mean- $Z$ and $l o-Z$ compositions. For $10-Z$ breast tissue $l o-Z$ adipose and mammary gland tissues were combined. The relative variation of $D_{\mathrm{w}, \mathrm{m}} / D_{\mathrm{m}, \mathrm{m}}$ caused by the variation of the composition of a given tissue within $\pm 1 \sigma$, labeled $\Delta D_{\mathrm{w}, \mathrm{m}} / D_{\mathrm{m}, \mathrm{m}}$ is given by: 


$$
\Delta D_{\mathrm{w}, \mathrm{m}} / D_{\mathrm{m}, \mathrm{m}}=\frac{\left(D_{\mathrm{w}, \mathrm{m}} / D_{\mathrm{m}, \mathrm{m}}\right)_{\text {low-z }}-\left(D_{\mathrm{w}, \mathrm{m}} / D_{\mathrm{m}, \mathrm{m}}\right)_{\mathrm{hi-z}}}{\left(D_{\mathrm{w}, \mathrm{m}} / D_{\mathrm{m}, \mathrm{m}}\right)_{\text {mean-z }}} \cdot 100 \% .
$$

It ranges from about $4 \%$ for Muscle up to $30 \%$ for Mammary gland. Interestingly, $\Delta D_{\mathrm{w}, \mathrm{m}} / D_{\mathrm{m}, \mathrm{m}}$ does not vary much from one source to the other. For Mammary gland (Muscle) it takes the values 33\% (4\%), 34\% (4\%) and 33\% (4\%) for the ${ }^{125} \mathrm{I},{ }^{103} \mathrm{Pd}$ and Axxent sources respectively. This means that all sources are equally sensitive to variations in tissue composition.

Table 4.2: $D_{w, m} / D_{m, m}$ values at $1 \mathrm{~cm}$ distance from the source for $\mathrm{hi}-Z$, mean-Z and $10-Z$ compositions for ${ }^{125} \mathrm{I},{ }^{103} \mathrm{Pd}$ and the Axxent source.

\begin{tabular}{llll}
\hline \hline & ${ }^{125} I$ & ${ }^{103} \mathrm{Pd}$ & Axxent \\
Tissue & $h i-Z-$ mean-Z-lo-Z & hi-Z-mean-Z-lo-Z & hi-Z - mean-Z-lo-Z \\
\hline Adipose & $1.52-1.66-1.83$ & $1.54-1.69-1.88$ & $1.52-1.66-1.84$ \\
Mammary gland & $1.08-1.24-1.48$ & $1.08-1.25-1.50$ & $1.08-1.24-1.49$ \\
Muscle & $0.95-0.97-0.99$ & $0.95-0.98-0.99$ & $0.95-0.97-0.99$ \\
Skin & $1.03-1.06-1.09$ & $1.03-1.07-1.10$ & $1.03-1.06-1.09$ \\
Breast A70G30 & $1.35-1.51-1.71$ & $1.36-1.53-1.75$ & $1.35-1.51-1.72$ \\
\hline \hline
\end{tabular}

\section{Clinical cases}

The ratio of PTV $D_{90(w, m)}$ (from the $D_{w, m}$ distribution) over PTV $D_{90(m, m)}$ (from the $D_{\mathrm{m}, \mathrm{m}}$ distribution) was calculated for each prostate and breast cancer patient. For each site the average value of the ratio and its standard deviation over four patients is presented in Table 4.3. We observe that $D_{90,(\mathrm{w}, \mathrm{m})} / D_{90,(\mathrm{~m}, \mathrm{~m})}$ has similar values as $D_{\mathrm{w}, \mathrm{m}} / D_{\mathrm{m}, \mathrm{m}}$ at $1 \mathrm{~cm}$ from point sources (Table 4.1). Prostate $A$ is nearly water equivalent and scoring $D_{\mathrm{w}, \mathrm{m}}$ or $D_{\mathrm{m}, \mathrm{m}}$ results in the same $D_{90}$ values. Using Prostate $C$ on the other hand results in a $7.5 \%$ difference. Breast tissue is much more sensitive, exhibiting a $53 \%$ difference between scoring schemes. The variation over the four patients is small, meaning that the difference in seed distribution over these four patients did not influence the ratio. This variation would be larger in reality: Table 4.2 shows that variations of tissue composition among patients influence the ratio significantly while in this work the tissue composition was kept constant for all four patients.

Table 4.3: Average $D_{90,(w, m)} / D_{90,(m, m)}$ and standard deviation over four patients for both prostate cases and breast cases.

\begin{tabular}{ccc}
\hline \hline Tissue in & $\left\langle D_{90,(\mathrm{w}, \mathrm{m})} / D_{90,(\mathrm{~m}, \mathrm{~m})}\right\rangle$ & $\begin{array}{c}\text { Absolute } \\
\text { ard deviation }\end{array}$ \\
\hline PTV contour & & \\
Prostate cases & 0.997 & 0.001 \\
Prostate A & 1.08 & 0.01 \\
Prostate C & & \\
Breast cases & 1.53 & 0.01 \\
Breast A70G30 & & \\
\hline \hline
\end{tabular}

Figure 4.6 shows PTV differential dose volume histograms (dDVH) of $D_{\mathrm{w}, \mathrm{m}}$ and $D_{\mathrm{m}, \mathrm{m}}$ distributions for one of the breast cancer patients studied. The dDVH of the $D_{\mathrm{w}, \mathrm{w}}$ 
distribution, which corresponds to the clinical TG-43 dose calculation, is added as reference. We observe that doses are generally highest in the $D_{\mathrm{w}, \mathrm{m}}$ distribution, given the lower attenuation of breast tissue than water. When scoring $D_{\mathrm{m}, \mathrm{m}}$, the effect of the decreased attenuation is counterbalanced by a lower absorption, yielding lower doses than TG-43.

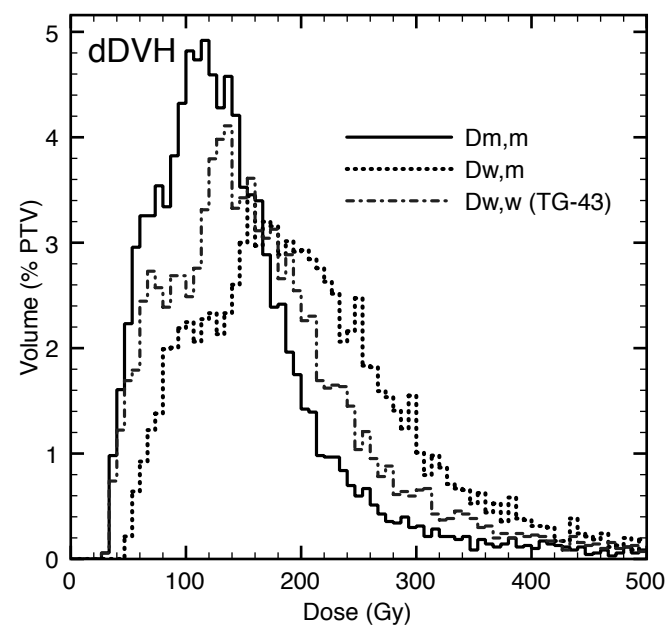

Figure 4.6: Comparison of differential DVH for the PTV of a clinical brachytherapy breast implant using ${ }^{103} \mathrm{Pd}$ seeds. Three dose distributions are investigated: $D_{w, m}, D_{m, m}$ and $D_{w, w}$ (which represents TG-43).

\section{DISCUSSION}

The large differences we have found between $D_{\mathrm{w}, \mathrm{m}}$ and $D_{\mathrm{m}, \mathrm{m}}$ for breast stress the importance of formulating guidelines for low-energy photon brachytherapy dose reporting. It is necessary to select the dose scoring method that is the most relevant from a clinical and physical standpoint. The differences reported in this work are much larger than what is observed in external photon beam radiotherapy, as expected by the important variations of $\mu_{\mathrm{en}} / \rho$ between tissues at low photon energies. Siebers et $a I^{9}{ }^{9}$, using small cavity theory with unrestricted stopping power ratios, reported differences of about $2 \%$ for ICRU soft tissue and lung using $6 \mathrm{MV}$ and $18 \mathrm{MV}$ linac beams. Soft bone, with a $Z_{\text {eff }}$ of 10.7 , an extreme case in the context of this work, shows only a $4 \%$ difference. In contrast we observe differences of up to $70 \%$ for adipose tissue. Our results agree with Rivard et al. ${ }^{14}$ who found a $25 \%$ difference at $20 \mathrm{keV}$ between $D_{\mathrm{w}, \mathrm{m}}$ and $D_{\mathrm{m}, \mathrm{m}}$ for ICRU 44 breast, which corresponds to our Mammary gland mean-Z (Table I, ${ }^{103} \mathrm{Pd}$ ). The vast majority of $\mathrm{MC}$ studies in brachytherapy have reported $D_{\mathrm{m}, \mathrm{m}}$ with no mention of $D_{\mathrm{w}, \mathrm{m}}$. 
This work is based on the assumptions of large cavity theory which definitely hold for uniform media but might not be applicable when considering the geometry of a human cell. Whether the whole cell or its nucleus is the actual radiation target is also an important factor affecting how well the dose scored in a voxel, which is in itself not a biologically relevant entity, relates to radiation response. The uncertainty on the composition of body tissues also affects the accuracy of conversion factors.

Table 4.4 shows $D_{90}$ values obtained for a specific breast case where both $D_{\mathrm{m}, \mathrm{m}}$ and $D_{\mathrm{w}, \mathrm{m}}$ were scored. In addition, $D_{\mathrm{w}, \mathrm{w}}$ is also shown (obtained when all voxels are water). The decrease of $D_{90}$ when going from $D_{\mathrm{w}, \mathrm{w}}$ to $D_{\mathrm{m}, \mathrm{m}}$ is consistent with the literature ${ }^{3}$ which reports $D_{\mathrm{m}, \mathrm{m}}$. Scoring $D_{\mathrm{w}, \mathrm{m}}$ reverses this trend and results in increased $D_{90}$ values. When transporting photons in breast tissue, the fluence reaching a scoring voxel is generally higher because breast has lower attenuation than water. When scoring $D_{\mathrm{w}, \mathrm{m}}$ this results in a higher dose in the voxel than for $D_{\mathrm{w}, \mathrm{w}}$. When scoring $D_{\mathrm{m}, \mathrm{m}}$, the lower $\left(\mu_{\mathrm{en}} / \rho\right)_{\mathrm{m}}$ of breast tissue decreases the energy absorbed in the voxel. This decrease is greater than the increase in photon fluence stemming from transport in breast tissue, leading to a dose which is lower than for $D_{\mathrm{w}, \mathrm{w}}$.

In Table 4.4 two compositions were studied, Breast A70G30 mean- $Z$ and $10-Z$ for which the values of $D_{\mathrm{w}, \mathrm{m}}$ and $D_{\mathrm{m}, \mathrm{m}}$ differ greatly. For $D_{\mathrm{w}, \mathrm{m}}$ going from mean-Z to $10-Z$ results in a $6 \% D_{90(w, m)}$ increase. For $D_{m, m}$ the effect is opposite, we observe an $8 \%$ $D_{90(m, m)}$ decrease going from mean- $Z$ to $l o-Z$. These differences can be interpreted as the dose calculation uncertainties caused by the variation of tissue composition.

Table 4.4: $D_{90}$ values for a specific breast implant for both dose reporting methods and for two breast compositions.

\begin{tabular}{cccc}
\hline \hline Breast A70G30 composition & $D_{90(\mathrm{w}, \mathrm{m})}$ & $D_{90(\mathrm{~m}, \mathrm{~m})}$ & $D_{90(\mathrm{w}, \mathrm{w})}$ \\
\cline { 2 - 4 } & & (\% prescription dose) & \\
\hline mean-Z & 135 & 89 & 103 \\
lo-Z & 143 & 82 & 103 \\
\hline \hline
\end{tabular}

To be able to put the two dose scoring quantities in perspective it is helpful to define some desired properties of a dose quantity:

1. A quantity that correlates with radiation response for similar cells in various tissues.

2. A quantity that can be easily calculated and implemented.

3. A quantity that does not degrade the accuracy of the calculated dose.

With respect to Property 1 , studies correlating treatment outcome to $D_{\mathrm{m}, \mathrm{m}}$ and $D_{\mathrm{w}, \mathrm{m}}$ would provide information and potentially definitive arguments as to which scoring method is preferred. Ideally a site where tissue composition varies significantly between patients and where information on composition can be extracted would be chosen for such a study. Property 2 is satisfied given the availability of MBDC algorithms. Given the uncertainties on tissue composition, Property 3 is not satisfied for either quantity: we see from Table IV that the potential calculation errors from assuming mean breast compositions are about the same order for $D_{\mathrm{w}, \mathrm{m}}(6 \%)$ as for $D_{\mathrm{m}, \mathrm{m}}(-8 \%)$. 
However, using MBDC to calculate $D_{\mathrm{w}, \mathrm{m}}$ or $D_{\mathrm{m}, \mathrm{m}}$ using mean tissue compositions is still an improvement over TG-43 $D_{\mathrm{w}, \mathrm{w}}$ calculations, which introduce larger errors (Table 4.4).

Given the upcoming release of the AAPM TG-186 report, which may issue recommendations concerning scoring medium, we refrain here from recommending a scoring approach.

\section{CONCLUSION}

MBDC algorithms allow dose calculations in various media and intrinsically provide dose to the local medium with transport in medium, $D_{\mathrm{m}, \mathrm{m}}$ and can also provide dose to a small mass of water with transport in medium, $D_{\mathrm{w}, \mathrm{m}}$. In this work these two scoring schemes were compared for a series of human tissues and radiation sources relevant for low energy brachytherapy. Differences of $-3 \%$ to $+70 \%$ can be observed between the two quantities depending on the scoring tissue, as expected from mass energy absorption coefficient ratios. The conversion factors from one scheme to the other do not vary more than $2 \%$ with distance from the source, even in the presence of important spectral shifts. The conversion factors are also very similar between low energy brachytherapy sources. Within a tissue type, variations of composition can influence the conversion factors up to $38 \%$. Conversion factors for clinical implants agree with those obtained in idealized geometries.

\section{ACKNOWLEDGEMENTS}

This work is supported by grant \#017133 of the Canadian Cancer Society (CCS) as well as from funding of the Canadian Breast Cancer Foundation - Ontario Chapter. GL is supported by a PGSD2 scholarship from the Natural Sciences and Engineering Research Council of Canada (NSERC). BR is supported by a Marie Curie Reintegration Grant (grant agreement no. PIRG05-GA-2009-247878 from FP7-PEOPLE-2009-RG). The authors would like to thank Lars Murrer, Esther Bloemen-van Gurp and Åsa Carlsson Tedgren for useful discussions.

\section{REFERENCES}

${ }^{1}$ R. Nath, L.L. Anderson, G. Luxton, K.A. Weaver, J.F. Williamson, and A.S. Meigooni, "Dosimetry of interstitial brachytherapy sources: recommendations of the AAPM Radiation Therapy Committee Task Group No. 43. American Association of Physicists in Medicine," Med Phys 22, 209-234 (1995).

${ }^{2}$ M.J. Rivard, et al., "Update of AAPM Task Group No. 43 Report: A revised AAPM protocol for brachytherapy dose calculations," Med Phys 31, 633-674 (2004). 
${ }^{3}$ H. Afsharpour, J.-P. Pignol, B. Keller, J.-F. Carrier, B. Reniers, F. Verhaegen, and L. Beaulieu, "Influence of breast composition and interseed attenuation in dose calculations for postimplant assessment of permanent breast ${ }^{103}$ Pd seed implant," Phys Med Biol 55, 4547-4561 (2010).

${ }^{4}$ G.S. Burns and D.E. Raeside, "The accuracy of single-seed dose superposition for $1-125$ implants," Med Phys 16, 627-631 (1989).

5J.F. Carrier, M. D'Amours, F. Verhaegen, B. Reniers, A.G. Martin, E. Vigneault, and L. Beaulieu, "Postimplant dosimetry using a Monte Carlo dose calculation engine: a new clinical standard," Int J Radiat Oncol Biol Phys 68, 1190-1198 (2007).

${ }^{6}$ O. Chibani, J.F. Williamson, and D. Todor, "Dosimetric effects of seed anisotropy and interseed attenuation for 103Pd and 125 I prostate implants," Med Phys 32, 2557-2566 (2005).

${ }^{7}$ R.M. Thomson, R.E. Taylor, and D.W. Rogers, "Monte Carlo dosimetry for 1251 and $103 \mathrm{Pd}$ eye plaque brachytherapy," Med Phys 35, 5530-5543 (2008).

${ }^{8}$ I.J. Chetty, et al., "Report of the AAPM Task Group No. 105: Issues associated with clinical implementation of Monte Carlo-based photon and electron external beam treatment planning," Med Phys 34, 4818-4853 (2007).

${ }^{9}$ J.V. Siebers, P.J. Keall, A.E. Nahum, and R. Mohan, "Converting absorbed dose to medium to absorbed dose to water for Monte Carlo based photon beam dose calculations," Phys Med Biol 45, 983-995 (2000).

${ }^{10}$ B.R. Walters, R. Kramer, and I. Kawrakow, "Dose to medium versus dose to water as an estimator of dose to sensitive skeletal tissue," Phys Med Biol 55, 4535-4546 (2010).

${ }^{11} \mathrm{H}$. Liu, P. Keall, and W. Hendee, "Dm rather than Dw should be used in Monte Carlo treatment planning," Med Phys 29, 922-924 (2002).

${ }^{12}$ ICRU, "Report 83: Prescribing, Recording, and Reporting Photon-Beam Intensity-Modulated Radiation Therapy (IMRT)," Journal of the ICRU 10, 63-66 (2010).

${ }^{13}$ A.E. Nahum, Cavity theory, stopping-power ratios, correction factors, in Clinical dosimetry measurements in radiotherapy. 2009, Medical Physics Pub.: Madison, WI.

${ }^{14}$ M.J. Rivard, L. Beaulieu, and F. Mourtada, "Enhancements to commissioning techniques and quality assurance of brachytherapy treatment planning systems that use model-based dose calculation algorithms," Med Phys 37, 2645-2658 (2010).

${ }^{15}$ A.S. Meigooni, J.A. Meli, and R. Nath, "A comparison of solid phantoms with water for dosimetry of 125 I brachytherapy sources," Med Phys 15, 695-701 (1988).

${ }^{16}$ N.S. Patel, S.T. Chiu-Tsao, J.F. Williamson, P. Fan, T. Duckworth, D. Shasha, and L.B. Harrison, "Thermoluminescent dosimetry of the Symmetra $125 \mathrm{I}$ model 125.506 interstitial brachytherapy seed," Med Phys 28, 1761-1769 (2001).

${ }^{17}$ S. Agostinelli, et al., "Geant4-a Simulation Toolkit," Nuclear Instruments \& Methods in Physics Research Section a-Accelerators Spectrometers Detectors and Associated Equipment 506, 250-303 (2003).

${ }^{18}$ D. Cullen, J.H. Hubbell, and L. Kissel, "EPDL97: the Evaluated Photon Data Library, 97 version," UCRL-50400 Vol. 6, Rev 5, (1997).

${ }^{19}$ S.T. Perkins, D.E. Cullen, and S.M. Seltzer, "Tables and graphs of electron-interaction crosssections from $10 \mathrm{eV}$ to $100 \mathrm{GeV}$ derived from the LLNL Evaluated Electron Data Library (EEDL)," UCRL-50400 Vol. 31, (1997).

${ }^{20}$ S.T. Perkins, D.E. Cullen, M.H. Chen, J.H. Hubbell, J. Rathkopf, and J. Scofield, "Table and graphs of atomic subshell and relaxation data derived from the LLNL Evaluated Atomic Data Library (EADL)," UCRL-50400 Vol. 30, (1997).

${ }^{21}$ J.F. Williamson, "Monte Carlo evaluation of kerma at a point for photon transport problems," Med Phys 14, 567-576 (1987).

${ }^{22}$ I. Kawrakow, "Accurate condensed history Monte Carlo simulation of electron transport. I. EGSnrc, the new EGS4 version," Med Phys 27, 485-498 (2000). 
${ }^{23}$ J.H. Hubbell and S.M. Seltzer, Tables of X-Ray Mass Attenuation Coefficients and Mass EnergyAbsorption Coefficients. 1996, NIST.

${ }^{24}$ E. Poon and et al., "BrachyGUI: an adjunct to an accelerated Monte Carlo photon transport code for patient-specific brachytherapy dose calculations and analysis," Journal of Physics: Conference Series 102, 012018 (2008).

${ }^{25}$ R.E. Taylor and D.W. Rogers, "An EGSnrc Monte Carlo-calculated database of TG-43 parameters," Med Phys 35, 4228-4241 (2008).

${ }^{26}$ M.J. Rivard, "Brachytherapy dosimetry parameters calculated for a $131 \mathrm{Cs}$ source," Med Phys 34, 754-762 (2007).

${ }^{27}$ G. Landry, et al., "Sensitivity of low energy brachytherapy Monte Carlo dose calculations to uncertainties in human tissue composition," Med Phy 37, 5188-5198 (2010).

${ }^{28}$ D. Liu, E. Poon, M. Bazalova, B. Reniers, M. Evans, T. Rusch, and F. Verhaegen, "Spectroscopic characterization of a novel electronic brachytherapy system," Phys Med Biol 53, 61-75 (2008).

${ }^{29}$ R.E. Taylor and D.W. Rogers, "More accurate fitting of $125 \mathrm{I}$ and $103 \mathrm{Pd}$ radial dose functions," Med Phys 35, 4242-4250 (2008).

${ }^{30}$ H.Q. Woodard and D.R. White, "The composition of body tissues," Br J Radiol 59, 1209-1218 (1986).

${ }^{31}$ B.R. Walters, I. Kawrakow, and D.W. Rogers, "History by history statistical estimators in the BEAM code system," Med Phys 29, 2745-2752 (2002).

${ }^{32}$ M.J. Yaffe, et al., "The myth of the 50-50 breast," Med Phys 36, 5437-5443 (2009).

${ }^{33}$ J.P. Pignol, E. Rakovitch, B.M. Keller, R. Sankreacha, and C. Chartier, "Tolerance and acceptance results of a palladium-103 permanent breast seed implant Phase I/II study," Int J Radiat Oncol Biol Phys 73, 1482-1488 (2009). 



\section{CHAPTER}

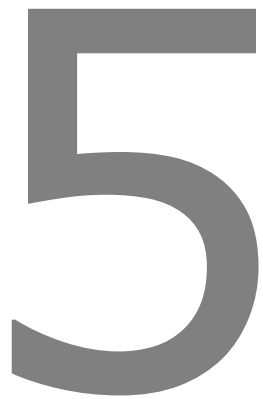

Simulation study on potential accuracy gains from dual energy CT tissue segmentation for low-energy brachytherapy Monte Carlo dose calculations

Guillaume Landry, Patrick Granton, Brigitte Reniers, Michel Ollers, Luc Beaulieu, Joachim E. Wildberger, Frank Verhaegen

Published in: Physics in Medicine and Biology 56(19):6257-6278, 2011. 


\section{ABSTRACT}

This work compares Monte Carlo (MC) dose calculations for ${ }^{125} \mathrm{I}$ and ${ }^{103} \mathrm{Pd}$ low dose rate (LDR) brachytherapy sources performed in virtual phantoms containing a series of human soft tissues of interest for brachytherapy. The geometries are segmented (tissue type and density assignment) based on simulated single energy computed tomography (SECT) and dual energy (DECT) images, as well as the all-water TG-43 approach. Accuracy is evaluated by comparison to a reference MC dose calculation performed in the same phantoms, where each voxel's material properties are assigned with exactly known values. The objective is to assess potential dose calculation accuracy gains from DECT. A CT imaging simulation package, ImaSim, is used to generate CT images of calibration and dose calculation phantoms at $80 \mathrm{kVp}, 120 \mathrm{kVp}$ and $140 \mathrm{kVp}$. From the high and low energy images electron density $\rho_{\mathrm{e}}$ and atomic number $Z$ are obtained using a DECT algorithm. Following a correction derived from scans of the calibration phantom, accuracy on $Z$ and $\rho_{\mathrm{e}}$ of $\pm 1 \%$ is obtained for all soft tissues with atomic number $Z \in[6,8]$ except lung. GEANT4 MC dose calculations based on DECT segmentation agreed with the reference within $\pm 4 \%$ for ${ }^{103} \mathrm{Pd}$, the most sensitive source to tissue misassignments. SECT segmentation with three tissue bins as well as the TG-43 approach showed inferior accuracy with errors of up to $20 \%$. Using seven tissue bins in our SECT segmentation brought errors within $\pm 10 \%$ for ${ }^{103} \mathrm{Pd}$. In general ${ }^{125}$ I dose calculations showed higher accuracy than ${ }^{103} \mathrm{Pd}$. Simulated image noise was found to decrease DECT accuracy by $3-4 \%$. Our findings suggest DECT based segmentation yields improved accuracy when compared to SECT segmentation with seven tissue bins in LDR brachytherapy dose calculation for the specific case of our nonanthropomorphic phantom. The validity of our conclusions for a clinical geometry as well as the importance of image noise in the tissue segmentation procedure deserve further experimental investigation. 


\section{INTRODUCTION}

To take tissue heterogeneities into account in brachytherapy dose calculations, model based dose calculation (MBDC) algorithms such as Monte Carlo (MC) simulations, collapsed cone convolution and Boltzmann transport equation solvers are considered by the active AAPM Radiation Therapy Committee Task Group 186 (TG-186) as potential successors to the Task Group 43 formalism (TG-43) and its updates ${ }^{1,2}$. Contrary to the water based TG-43 approach, these MBDC algorithms can model non-water heterogeneities including assorted tissue compositions and interseed attenuation important in low dose rate (LDR) brachytherapy and electronic brachytherapy sources (EBS), which emit a spectrum of bremsstrahlung and characteristic x-ray photons. It has been shown that ignoring tissue composition and interseed attenuation effects can lead to significant dose calculation errors in the low energy range of these photon sources (from a few to about $50 \mathrm{keV})^{3-8}$.

Accurate MBDC below $50 \mathrm{keV}$ are contingent upon correct tissue type identification. Because of the important proportion of photoelectric photon interactions, the elemental composition of tissues strongly determines the dose deposition behavior. Our previous work has shown that even assuming mean elemental compositions can lead to significant dose calculation errors ${ }^{9}$, thus patient-specific tissue identification suggests superior dose calculation accuracy. In current clinical practice, quantitative information on tissues is determined by conventional single energy CT (SECT) in the form of attenuation coefficients (often expressed by Hounsfield Units, HU). From these, mass or electron densities are derived using empirical relationships. The current standard for heterogeneous dose calculations, which are almost exclusively used in external beam therapy, is based on these densities and does not account for tissue type $^{10}$. Tissue type identification based on SECT measurement of the linear attenuation coefficient has been shown to lead to MC dose calculation errors in the megavoltage photon and electron energy range ${ }^{11}$. Because of the strong dependence of the photoelectric cross sections on the tissue's atomic number, such errors are expected to be amplified in the low energy range.

By measuring the linear attenuation coefficient at two well-separated photon energies, dual energy computed tomography (DECT) provides a means to express it in a two parameter model ${ }^{12}$. The parametric model extracts information pertaining to the elemental composition of a mixture (atomic numbers, $Z$ ) in addition to its electron density $\left(\rho_{\mathrm{e}}\right)$. Bazalova et al. ${ }^{13,14}$ adapted the synchrotron based approach of Torikoshi et $a .^{15}$ to determine $\mathrm{MC}$ dose calculation errors for both megavoltage and kilovoltage radiation therapy using DECT information of a phantom with known composition and density. In their study, they showed that DECT improved dose calculation accuracy when compared to SECT. Segmentation (the process of assigning tissue type and density) based on SECT caused dose calculation errors as high as $17 \%$ while DECT segmentation brought those errors within $1 \%$ for a $250 \mathrm{kVp}$ beam. Yang et al. ${ }^{16}$ used the same formalism to calculate patient-specific proton stopping power ratios in a theoretical study, concluding that DECT has a theoretical advantage over SECT. Mahnken et al. ${ }^{17}$ used a similar method ${ }^{18,19}$ to characterize body fluids. Since clinical 
DECT systems are becoming available for diagnostic purposes, there is a need to quantify their potential to identify tissues for low energy brachytherapy MBDC.

In this report, a study was performed with simulated images in which tissue segmentation schemes based on virtual DECT and SECT images were employed to perform MC dose calculations in mathematical phantoms comprising soft tissues of interest in brachytherapy, using ${ }^{103} \mathrm{Pd}$ and ${ }^{125} \mathrm{I}$ implants. Accuracy was evaluated by comparing to a reference dose calculation performed in a geometry with exactly known tissue compositions.

\section{MATERIALS AND METHODS}

\section{CT image simulation}

In this work CT images were generated with ImaSim ${ }^{20}$. This program is based on SpekCalc, a validated $x$-ray spectrum generation tool ${ }^{21-23}$. The program calculates $x$-ray projection images using ray tracing for a fan beam CT geometry from a point source. The object attenuation $A$ measured in a specific detector element is given by:

$$
A=\frac{\int_{\text {spectrum }} S(E) D(E) e^{-\int \mu(r, E) d r} d E}{\int_{\text {spectrum }} S(E) D(E) d E}
$$

where $S(E)$ is the x-ray photon spectrum, $D(E)$ is the detector response (and would be $D(E)=E$ in the case of a perfect energy integrating detector) and $\mu(r, E)$ is the linear attenuation coefficient at the position $\mathbf{r}$ in the object and at photon energy $E$. The integral in the exponent is along the line / defined by the point source and the centre of the detector element for which the object attenuation is calculated. Attenuation coefficients are from the NIST XCOM database ${ }^{24}$. Following sinogram re-binning to parallel geometry ${ }^{25}$ a filtered back projection reconstruction is performed with either a Shepp Logan or a cosine filter. A cupping correction is applied for cylindrical phantoms of radius $R$. The correction, applied on projections, is such that the reconstructed image of a water phantom of radius $R$ would have a flat CT number profile.

\section{DECT algorithm}

This work builds upon the approach of Bazalova et al. ${ }^{13,14}$ to obtain atomic number $Z$ and electron density $\rho_{\mathrm{e}}$ from pairs of CT images taken at two different tube potentials. The algorithm relies on knowledge of the CT scanner's photon spectra and detector response. We modified Bazalova et al.'s algorithm slightly by using a different formulation for the atomic number of water $Z_{w}$. This quantity is necessary to convert scanner 
generated Hounsfield units back into linear attenuation coefficients. The details of the algorithm are presented in the Appendix.

The atomic number $Z$ provided by the algorithm is an effective atomic number, i.e. it is a mixture specific quantity, which in conjunction with $\rho_{\mathrm{e}}$ is used to calculate the linear attenuation coefficient of the mixture using the parameterisation presented in the Appendix. Thus $Z$ is specific to this parameterisation and is not necessarily equivalent to traditional formulations of effective atomic number $Z_{\text {eff }}$ such as Johns and Cunningham's ${ }^{26}$.

\section{Image simulation inputs}

Three photon spectra from a $10^{\circ}$ tungsten anode were generated: $80 / 140 \mathrm{kVp}$ with 10 $\mathrm{mm}$ Al filtration for DECT simulations and $120 \mathrm{kVp}$ with $7 \mathrm{~mm}$ Al filtration for SECT simulations; they are shown in figure 1 . These were chosen to represent generic SECT and DECT scanners. A heavier filtration was chosen for DECT since beam hardening effects can negatively impact accuracy ${ }^{13}$. The detector response for all scans was taken from Heismann and Balda ${ }^{18}$ and is for a $1.4 \mathrm{~mm} \mathrm{Gd}_{2} \mathrm{O}_{2} \mathrm{~S}$ scintillator CT detector used in clinical DECT systems.

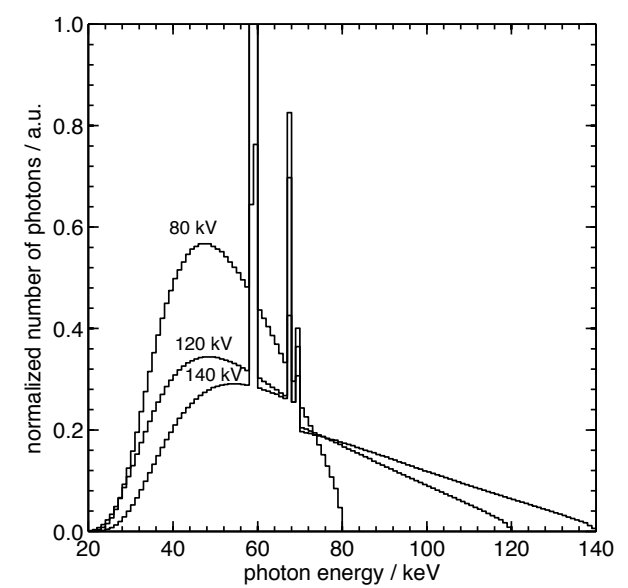

Figure 5.1: X-ray photon spectra used in this study. All spectra normalized to unit area under the curve.

We considered three mathematical phantoms. One is the calibration phantom; it was used to assess the SECT and DECT system performance. The calibration phantom contains tissue mimicking inserts: RMI tissue substitutes (Gammex, Middleton, WI) as well as solutions from Verhaegen and Devic ${ }^{11}$. The phantom geometry is shown in Figure 5.2 and information on insert composition and density is in Table 5.1.

Figure 5.3 shows the geometry of the dose calculation phantoms. These phantoms contain human tissues of interest for low energy brachytherapy. The first phantom contains tissues related to the female breast (Table 5.2, containing ${ }^{103} \mathrm{Pd}$ seeds), while 
the second contains tissues related to the prostate (Table 5.3 , containing ${ }^{125} \mathrm{I}$ seeds). Some tissues are found in both inner and outer rings of inserts, which represent the target and organs at risk respectively. The prostate phantom contains some of the tissues found in the breast phantom, such as adipose, muscle, lung and rib. This is to assess the differences between ${ }^{103} \mathrm{Pd}$ and ${ }^{125} \mathrm{I}$, although a detailed analysis of source differences is beyond the scope of the paper. Images of these two phantoms were used for dose calculations, the details of which are given in a later section. The elemental compositions of tissues are from ${ }^{27-29}$ and mean- $Z, 10-Z$ and $h i-Z$ denote mean and mean \pm one standard deviation tissue compositions for one tissue type, as defined in Woodard and White ${ }^{28}$ and sorted by effective atomic number $Z_{\text {eff. }} Z_{\text {eff }}$ is different than the $Z$ provided by our DECT approach, its definition is given in a later section. The Breast A70G30 composition is obtained by combining $30 \%$ Gland mean- $Z$ and $70 \%$ Adipose mean-Z.

Each phantom was scanned using the three spectra with 2048 projections and 512 detector elements. It is important to note that image resolution is not one of the parameters considered in this study. For this reason we allowed the same number of detectors to cover the width of both the calibration and dose calculation phantoms. Images were reconstructed onto a 512×512 image grid with a Shepp Logan filter.

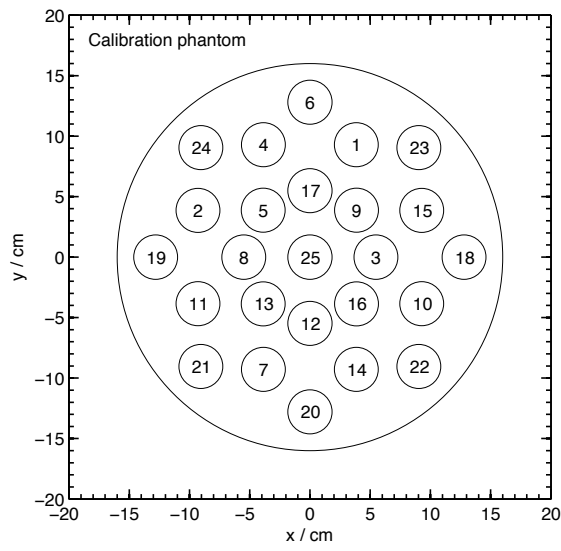

Figure 5.2: Cross sectional view of the calibration phantom. Drawn to scale. The phantom's body is solid water. Identification numbers correspond to tissues (Table 5.1).

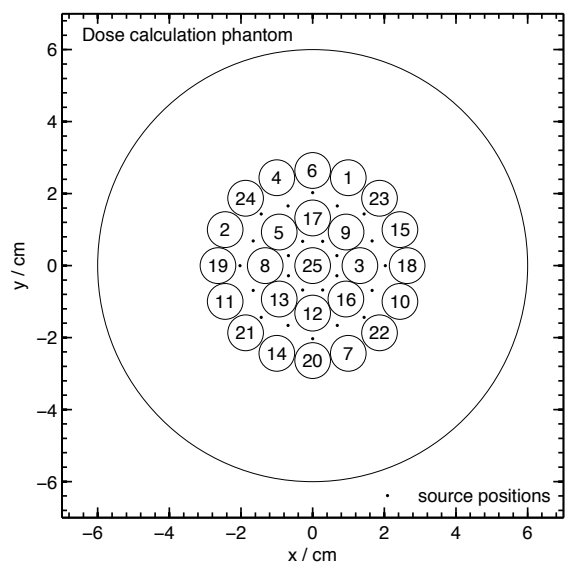

Figure 5.3: Cross sectional view of the dose calculation phantom. Dots indicate the position of radiation sources for dose calculation. Drawn to scale. The phantom's body is female soft tissue for the breast dose calculation phantom and male soft tissue for the prostate dose calculation phantom. Identification numbers correspond to tissues (Tables 5.2, 5.3). 
Table 5.1 Elemental compositions by weight fraction of tissue substitutes used in the calibration phantom. Identification number (ID) corresponds to inserts in the calibration phantom. Data sorted according to $Z_{\text {eff. }}$

\begin{tabular}{|c|c|c|c|c|c|c|c|c|c|}
\hline \multirow[b]{2}{*}{ ID } & \multirow[b]{2}{*}{ Tissue substitute } & $\mathrm{H}$ & $\mathrm{C}$ & $\mathrm{N}$ & 0 & $Z>8$ & $\rho$ & $\rho_{\mathrm{e}} / \rho_{\mathrm{e}, \mathrm{w}}$ & $Z_{\text {eff }}$ \\
\hline & & \multicolumn{5}{|c|}{$\%$ by weight } & \multicolumn{3}{|l|}{$\mathrm{g} \cdot \mathrm{cm}^{-3}$} \\
\hline 15 & Polyethylene & 14.4 & 85.6 & 0 & 0 & 0 & 0.920 & 0.945 & 5.50 \\
\hline 14 & SR2 brain & 10.8 & 72.5 & 1.7 & 14.9 & $\mathrm{Cl}(0.1)$ & 1.045 & 1.040 & 6.10 \\
\hline 1 & AP6 adipose & 9.1 & 72.3 & 2.3 & 16.3 & $\mathrm{Cl}(0.1)$ & 0.920 & 0.903 & 6.20 \\
\hline 6 & CB4 & 8.2 & 70.2 & 2.7 & 18.8 & $\mathrm{Cl}(0.1)$ & 1.155 & 1.123 & 6.29 \\
\hline 7 & $\mathrm{CB} 3$ resin & 11.3 & 74.2 & 1.6 & 12.0 & $\mathrm{Cl}(0.9)$ & 1.020 & 1.020 & 6.37 \\
\hline 9 & Ethanol (0.81) & 13.03 & 49.54 & 0 & 37.43 & 0 & 0.810 & 0.823 & 6.49 \\
\hline 2 & Lucite & 8.1 & 60.0 & 0 & 32.0 & 0 & 1.180 & 1.148 & 6.53 \\
\hline 3 & Ethanol (0.85) & 12.66 & 39.63 & 0 & 47.71 & 0 & 0.850 & 0.861 & 6.72 \\
\hline 4 & BR12 breast & 8.6 & 70.1 & 2.3 & 17.9 & $\mathrm{Cl}(0.1), \mathrm{Ca}(1.0)$ & 0.980 & 0.957 & 6.96 \\
\hline 16 & Ethanol (0.94) & 11.93 & 19.81 & 0 & 68.26 & 0 & 0.940 & 0.946 & 7.12 \\
\hline 12 & Ethanol (0.977) & 11.37 & 4.95 & 0 & 83.67 & 0 & 0.977 & 0.978 & 7.39 \\
\hline 5 & Water & 11.19 & 0 & 0 & 88.81 & 0 & 1.000 & 1.000 & 7.48 \\
\hline 25 & LN450 lung & 8.5 & 59.6 & 2.0 & 18.1 & $\mathrm{Mg}(11.2), \mathrm{Si}(0.6), \mathrm{Cl}(0.1)$ & 0.450 & 0.439 & 7.56 \\
\hline 13 & LN300 lung & 8.5 & 59.4 & 2.0 & 18.1 & $\mathrm{Mg}(11.2), \mathrm{Si}(0.8), \mathrm{Cl}(0.1)$ & 0.300 & 0.292 & 7.59 \\
\hline 17 & $\mathrm{NaCl}(1.001)$ & 11.08 & 0 & 0 & 87.93 & $\mathrm{Na}(0.39), \mathrm{Cl}(0.6)$ & 1.001 & 1.000 & 7.66 \\
\hline 10 & Solid Water & 8.0 & 67.3 & 2.4 & 19.9 & $\mathrm{Cl}(0.1), \mathrm{Ca}(2.3)$ & 1.015 & 0.985 & 7.73 \\
\hline 11 & LV1 RMI & 8.1 & 67.0 & 2.5 & 20.0 & $\mathrm{Cl}(0.1), \mathrm{Ca}(2.3)$ & 1.039 & 1.010 & 7.73 \\
\hline 8 & $\mathrm{CaCl}_{2}(1.00)$ & 11.08 & 0 & 0 & 87.93 & $\mathrm{Cl}(0.63), \mathrm{Ca}(0.36)$ & 1.000 & 0.999 & 7.82 \\
\hline 18 & $\mathrm{CB} 2-10 \% \mathrm{CaCO}_{3}$ & 8.6 & 65.3 & 2.7 & 19.2 & $\mathrm{Cl}(0.1), \mathrm{Ca}(4.0)$ & 1.170 & 1.141 & 8.46 \\
\hline 19 & IB3 inner bone & 6.7 & 55.6 & 2.0 & 23.5 & $\mathrm{P}(3.2), \mathrm{Cl}(0.1), \mathrm{Ca}(8.9)$ & 1.133 & 1.086 & 10.44 \\
\hline 23 & B200 bone mineral & 6.6 & 55.5 & 2.0 & 23.6 & $\mathrm{P}(3.2), \mathrm{Cl}(0.1), \mathrm{Ca}(8.9)$ & 1.145 & 1.095 & 10.44 \\
\hline 24 & $\mathrm{CB} 2-30 \% \mathrm{CaCO}_{3}$ & 6.7 & 53.5 & 2.1 & 25.6 & $\mathrm{Cl}(0.1), \mathrm{Ca}(12.0)$ & 1.340 & 1.285 & 10.91 \\
\hline 21 & $\mathrm{CB} 2-50 \% \mathrm{CaCO}_{3}$ & 4.8 & 41.6 & 1.5 & 32.0 & $\mathrm{Cl}(0.1), \mathrm{Ca}(20)$ & 1.560 & 1.470 & 12.55 \\
\hline 20 & SB3 cortical bone & 3.4 & 31.4 & 1.8 & 36.5 & $\mathrm{Cl}(0.04), \mathrm{Ca}(26.8)$ & 1.819 & 1.691 & 13.65 \\
\hline
\end{tabular}

Table 5.2 Elemental compositions by weight fraction of tissues for the breast dose calculation phantom. Identification number (ID) corresponds to inserts in the phantom. Data sorted according to $Z_{\text {eff. }}$.

\begin{tabular}{|c|c|c|c|c|c|c|c|c|c|}
\hline \multirow[b]{2}{*}{ ID } & \multirow[b]{2}{*}{ Tissue } & $\mathrm{H}$ & $\mathrm{C}$ & $\mathrm{N}$ & $\mathrm{O}$ & $Z>8$ & $\rho$ & $\rho_{\mathrm{e}} / \rho_{\mathrm{e}, \mathrm{w}}$ & $Z_{\text {eff }}$ \\
\hline & & \multicolumn{5}{|c|}{$\%$ by weight } & \multicolumn{3}{|l|}{$\mathrm{g} \cdot \mathrm{cm}^{-3}$} \\
\hline 17,21 & Adipose lo-Z & 11.6 & 68.1 & 0.2 & 19.8 & $\mathrm{Na}(0.1), \mathrm{S}(0.1), \mathrm{Cl}(0.1)$ & 0.930 & 0.932 & 6.23 \\
\hline 3,6 & Adipose mean-Z & 11.4 & 59.8 & 0.7 & 27.8 & $\mathrm{Na}(0.1), \mathrm{S}(0.1), \mathrm{Cl}(0.1)$ & 0.950 & 0.951 & 6.42 \\
\hline 9,22 & Adipose hi-Z & 11.2 & 51.7 & 1.3 & 35.5 & $\mathrm{Na}(0.1), \mathrm{S}(0.1), \mathrm{Cl}(0.1)$ & 0.970 & 0.969 & 6.60 \\
\hline 13,20 & Breast A70G30 & 11.16 & 51.82 & 1.39 & 35.27 & $\mathrm{Na}(0.1), \mathrm{P}(0.03), \mathrm{S}(0.13), \mathrm{Cl}(0.1)$ & 0.970 & 0.969 & 6.61 \\
\hline 8,24 & Gland lo-Z & 10.9 & 50.6 & 2.3 & 35.8 & $\mathrm{Na}(0.1), \mathrm{P}(0.1), \mathrm{S}(0.1), \mathrm{Cl}(0.1)$ & 0.990 & 0.987 & 6.65 \\
\hline 12,18 & Breast A30G70 & 10.84 & 41.18 & 2.31 & 45.23 & $\mathrm{Na}(0.1), \mathrm{P}(0.07), \mathrm{S}(0.17), \mathrm{Cl}(0.1)$ & 1.000 & 0.996 & 6.85 \\
\hline 16,19 & Gland mean-Z & 10.6 & 33.2 & 3.0 & 52.7 & $\mathrm{Na}(0.1), \mathrm{P}(0.1), \mathrm{S}(0.2), \mathrm{Cl}(0.1)$ & 1.020 & 1.014 & 7.02 \\
\hline 25 & $\begin{array}{l}\text { Average female } \\
\text { soft tissue }\end{array}$ & 10.6 & 31.5 & 2.4 & 54.7 & $\mathrm{Na}(0.1), \mathrm{P}(0.2), \mathrm{S}(0.2), \mathrm{Cl}(0.1), \mathrm{K}(0.2)$ & 1.020 & 1.012 & 7.17 \\
\hline 7 & Skin lo-Z & 10.0 & 25.0 & 4.6 & 59.4 & $\mathrm{Na}(0.2), \mathrm{P}(0.1), \mathrm{S}(0.3), \mathrm{Cl}(0.3), \mathrm{K}(0.1)$ & 1.090 & 1.078 & 7.31 \\
\hline 5,23 & Gland hi-Z & 10.2 & 15.8 & 3.7 & 69.8 & $\mathrm{Na}(0.1), \mathrm{P}(0.1), \mathrm{S}(0.2), \mathrm{Cl}(0.1)$ & 1.060 & 1.050 & 7.33 \\
\hline 11 & Skin mean-Z & 10.0 & 20.4 & 4.2 & 64.5 & $\mathrm{Na}(0.2), \mathrm{P}(0.1), \mathrm{S}(0.2), \mathrm{Cl}(0.3), \mathrm{K}(0.1)$ & 1.090 & 1.078 & 7.37 \\
\hline 14 & Skin hi-Z & 10.1 & 15.8 & 3.7 & 69.5 & $\mathrm{Na}(0.2), \mathrm{P}(0.1), \mathrm{S}(0.2), \mathrm{Cl}(0.3), \mathrm{K}(0.1)$ & 1.090 & 1.079 & 7.44 \\
\hline 4 & Muscle lo-Z & 10.1 & 17.1 & 3.6 & 68.1 & $\mathrm{Na}(0.1), \mathrm{P}(0.2), \mathrm{S}(0.3), \mathrm{Cl}(0.1), \mathrm{K}(0.4)$ & 1.050 & 1.039 & 7.53 \\
\hline 10 & Muscle mean-Z & 10.2 & 14.3 & 3.4 & 71.0 & $\mathrm{Na}(0.1), \mathrm{P}(0.2), \mathrm{S}(0.3), \mathrm{Cl}(0.1), \mathrm{K}(0.4)$ & 1.050 & 1.040 & 7.57 \\
\hline 1 & Lung & 10.3 & 10.5 & 3.1 & 74.9 & $\mathrm{Na}(0.2), \mathrm{P}(0.2), \mathrm{S}(0.3), \mathrm{Cl}(0.3), \mathrm{K}(0.2)$ & 0.260 & 0.258 & 7.60 \\
\hline 2 & Muscle hi-Z & 10.2 & 11.2 & 3.0 & 74.5 & $\mathrm{Na}(0.1), \mathrm{P}(0.2), \mathrm{S}(0.3), \mathrm{Cl}(0.1), \mathrm{K}(0.4)$ & 1.050 & 1.040 & 7.62 \\
\hline 15 & Rib & 6.4 & 26.3 & 3.9 & 43.6 & $\begin{array}{l}\mathrm{Na}(0.1), \mathrm{Mg}(0.1), \mathrm{P}(6.0), \mathrm{S}(0.3), \mathrm{Cl}(0.1) \\
\mathrm{K}(0.1), \mathrm{Ca}(13.1)\end{array}$ & 1.410 & 1.347 & 11.8 \\
\hline
\end{tabular}


Table 5.3 Elemental compositions by weight fraction of tissues for the prostate dose calculation phantom. Identification number (ID) corresponds to inserts in the phantom. Data sorted according to $Z_{\text {eff. }}$.

\begin{tabular}{|c|c|c|c|c|c|c|c|c|c|}
\hline \multirow[b]{2}{*}{ ID } & \multirow[b]{2}{*}{ Tissue } & $\mathrm{H}$ & $\mathrm{C}$ & $\mathrm{N}$ & $\mathrm{O}$ & $Z>8$ & $\rho$ & $\rho_{\mathrm{e}} / \rho_{\mathrm{e}, \mathrm{w}}$ & $Z_{\text {eff }}$ \\
\hline & & \multicolumn{5}{|c|}{$\%$ by weight } & \multicolumn{3}{|l|}{$\mathrm{g} \cdot \mathrm{cm}^{-3}$} \\
\hline 24 & Adipose lo-Z & 11.6 & 68.1 & 0.2 & 19.8 & $\mathrm{Na}(0.1), \mathrm{S}(0.1), \mathrm{Cl}(0.1)$ & 0.930 & 0.932 & 6.23 \\
\hline 23 & Adipose mean-Z & 11.4 & 59.8 & 0.7 & 27.8 & $\mathrm{Na}(0.1), \mathrm{S}(0.1), \mathrm{Cl}(0.1)$ & 0.950 & 0.951 & 6.42 \\
\hline 18 & Adipose hi-Z & 11.2 & 51.7 & 1.3 & 35.5 & $\mathrm{Na}(0.1), \mathrm{S}(0.1), \mathrm{Cl}(0.1)$ & 0.970 & 0.969 & 6.60 \\
\hline $\begin{array}{l}12,14, \\
4\end{array}$ & $\begin{array}{l}\text { Average male soft } \\
\text { tissue }\end{array}$ & 10.5 & 25.6 & 2.7 & 60.2 & $\mathrm{Na}(0.1), \mathrm{P}(0.2), \mathrm{S}(0.3), \mathrm{Cl}(0.2), \mathrm{K}(0.2)$ & 1.030 & 1.023 & 7.32 \\
\hline 5,7 & Prostate C & 10.5 & 25.6 & 2.7 & 60.2 & $\mathrm{Na}(0.1), \mathrm{P}(0.2), \mathrm{S}(0.3), \mathrm{Cl}(0.2), \mathrm{K}(0.2)$ & 1.040 & 1.033 & 7.32 \\
\hline 9,11 & Rectum & 10.6 & 11.5 & 2.2 & 75.1 & $\mathrm{Na}(0.1), \mathrm{P}(0.1), \mathrm{S}(0.1), \mathrm{Cl}(0.2), \mathrm{K}(0.1)$ & 1.030 & 1.024 & 7.45 \\
\hline 2,25 & Water & 11.19 & 0 & 0 & 88.81 & 0 & 1.000 & 1.000 & 7.48 \\
\hline 17,19 & Prostate A & 10.5 & 8.9 & 2.5 & 77.4 & $\mathrm{Na}(0.2), \mathrm{P}(0.1), \mathrm{S}(0.2), \mathrm{K}(0.2)$ & 1.040 & 1.033 & 7.50 \\
\hline 20 & Muscle lo-Z & 10.1 & 17.1 & 3.6 & 68.1 & $\mathrm{Na}(0.1), \mathrm{P}(0.2), \mathrm{S}(0.3), \mathrm{Cl}(0.1), \mathrm{K}(0.4)$ & 1.050 & 1.039 & 7.53 \\
\hline 3,6 & Muscle mean-Z & 10.2 & 14.3 & 3.4 & 71 & $\mathrm{Na}(0.1), \mathrm{P}(0.2), \mathrm{S}(0.3), \mathrm{Cl}(0.1), \mathrm{K}(0.4)$ & 1.050 & 1.040 & 7.57 \\
\hline 21 & Lung & 10.3 & 10.5 & 3.1 & 74.9 & $\mathrm{Na}(0.2), \mathrm{P}(0.2), \mathrm{S}(0.3), \mathrm{Cl}(0.3), \mathrm{K}(0.2)$ & 0.260 & 0.258 & 7.60 \\
\hline 16,1 & Muscle hi-Z & 10.2 & 11.2 & 3.0 & 74.5 & $\mathrm{Na}(0.1), \mathrm{P}(0.2), \mathrm{S}(0.3), \mathrm{Cl}(0.1), \mathrm{K}(0.4)$ & 1.050 & 1.040 & 7.62 \\
\hline 13,15 & Bladder empty & 10.5 & 9.6 & 2.6 & 76.1 & $\mathrm{Na}(0.2), \mathrm{P}(0.2), \mathrm{S}(0.2), \mathrm{Cl}(0.3), \mathrm{K}(0.3)$ & 1.040 & 1.033 & 7.63 \\
\hline 8,10 & Bladder full & 10.8 & 3.5 & 1.5 & 83.0 & $\mathrm{Na}(0.3), \mathrm{P}(0.1), \mathrm{S}(0.1), \mathrm{Cl}(0.5), \mathrm{K}(0.2)$ & 1.030 & 1.026 & 7.69 \\
\hline 22 & Rib & 6.4 & 26.3 & 3.9 & 43.6 & $\begin{array}{l}\mathrm{Na}(0.1), \quad \mathrm{Mg}(0.1), \quad \mathrm{P}(6.0), \quad \mathrm{S}(0.3), \\
\mathrm{Cl}(0.1), \mathrm{K}(0.1), \mathrm{Ca}(13.1)\end{array}$ & 1.410 & 1.347 & 11.8 \\
\hline
\end{tabular}

Image noise was considered by adding Poisson noise to projections. The number of un-attenuated $x$-ray quanta reaching a detector element were adjusted to yield a standard deviation of $7 \mathrm{HU}$ for a $1 \mathrm{~cm}$ diameter ROI located at the centre of a $512 \times 512$ image of the dose calculation phantom for each $\mathrm{kVp}$. For simulations involving noise a cosine reconstruction filter was used instead of the Shepp Logan filter; it was chosen since it yields lower noise levels (7 HU for cosine vs. 12 HU for Shepp Logan).

\section{SECT density and tissue assignment}

From the $120 \mathrm{kVp}$ image of the calibration phantom two image segmentation schemes were devised. Both used the same two segment linear $\rho-\mathrm{HU}$ relationship established by fitting the simulated $\rho-\mathrm{HU}$ data, shown in Figure 5.4. The first segmentation scheme was based on three tissues (SECT-3). Since segmentation schemes were used for both breast and prostate dose calculation phantom images, male and female variants were devised containing prostate and breast specific tissue bins. The second segmentation scheme comprised 7 tissues (SECT-7). The HU range corresponding to soft tissue in the SECT-3 segmentation was split into five bins for the SECT-7 segmentation. The narrow bins were $50 \mathrm{HU}$ wide. The width of the narrow bins was chosen to cover the $95 \%$ confidence interval, assuming $12 \mathrm{HU}$ for the standard deviation of noisy measurements. For the prostate phantom, no skin tissue is present; for this reason the skin hi-Z bin has been appended to the muscle hi-Z bin, for a total of 6 tissues. The SECT-7 notation was kept nonetheless. 


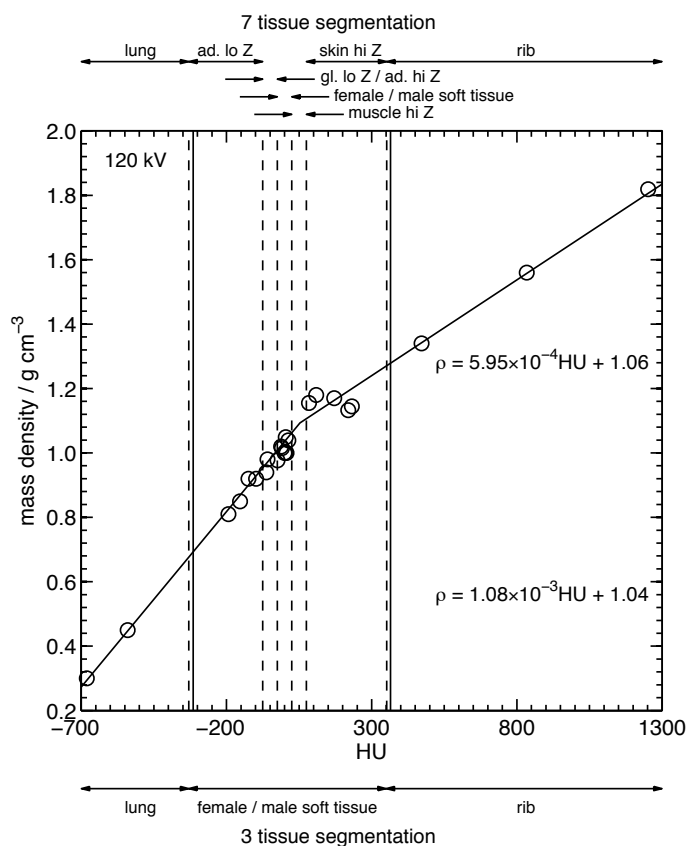

Figure 5.4: SECT segmentation schemes. Both schemes use the same piecewise linear $\rho-H U$ relation shown. Vertical solid lines indicate the bin edges for tissue types of the SECT-3 segmentation while the vertical dashed lines indicate those for the SECT-7 segmentation. The solid lines adjacent dashed lines are shifted by $+15 \mathrm{HU}$ for visualisation. Data points are from a $120 \mathrm{kVp}$ image of the calibration phantom. When two tissues are given for an interval, the first one is for the breast phantom and the second one for the prostate phantom.

\section{DECT density and tissue assignment}

DECT images of the dose calculation phantoms were first resampled to $256 \times 256$, averaging 4 pixels. The algorithm described above was then applied, yielding $Z$ and $\rho_{\mathrm{e}}$ maps. Fitting mass density $\rho$ to relative electron density $\rho_{\mathrm{e}} / \rho_{\mathrm{e}, \mathrm{w}}$, using the data (from Tables 5.2 and 5.3) on human tissues considered in this study (lung excluded), yields $\rho$ $=1.177 \times \rho_{\mathrm{e}} / \rho_{\mathrm{e}, \mathrm{w}}-0.174$ (in $\mathrm{gcm}^{-3}$ ). This equation was consequently used to convert the $\rho_{\mathrm{e}}$ map to a $\rho$ map used in dose calculations. To assign tissue type a correspondence was established between $Z$ values provided by our algorithm and the effective atomic number $Z_{\text {eff }}$ defined as:

$$
Z_{\text {eff }}=\left(\frac{\sum_{i} f_{i} \rho_{\mathrm{e}, i} Z_{i}^{n}}{\sum_{i} f_{i} \rho_{\mathrm{e}, i}}\right)^{1 / n}
$$


where $f_{i}$ is the mass fraction of element $i$. This formulation is from Johns and Cunningham ${ }^{26}$ where they recommend using $n=3.5$. In this work we have decided to use the DECT generated $Z$ map of the calibration phantom to find the exponent $n$ that minimizes the sum of squared differences between $Z_{\text {eff }}$ and $Z$, as suggested in Yang et al. ${ }^{16}$ Following this, $n=3.31$ was used to calculate $Z_{\text {eff }}$ for the human tissues of the dose calculation phantoms. These values are found in Tables 5.2 and 5.3. To assign a tissue composition to a voxel, the Euclidian distance between $\left[Z_{\mathrm{eff}} / Z_{\mathrm{eff}, \mathrm{w}}, \rho_{\mathrm{e}} / \rho_{\mathrm{e}, \mathrm{w}}\right]$ (from reference tissue compositions) and the voxel's DECT obtained $\left[Z / Z_{\mathrm{w}}, \rho_{\mathrm{e}} / \rho_{\mathrm{e}, \mathrm{w}}\right]$ was calculated. The reference tissue minimizing the Euclidian distance was assigned to the voxel. The normalization by water was to avoid unequal weighing of the parameters.

When performing tissue composition segmentation from noisy data a different distance is minimized: the Mahalanobis distance $L_{M}$ between vectors $\mathbf{X}=\left[Z_{\text {eff }} / Z_{\text {eff,w, }}\right.$, $\left.\rho_{\mathrm{e}} / \rho_{\mathrm{e}, \mathrm{w}}\right]$ and $\mathbf{X}_{\text {mes }}=\left[Z / Z_{\mathrm{w}}, \rho_{\mathrm{e}} / \rho_{\mathrm{e}, \mathrm{w}}\right]$ (DECT measurements) which is calculated by:

$$
L_{\mathrm{M}}=\left[\left(\mathbf{X}-\mathbf{X}_{\text {mes }}\right) \cdot \mathbf{C}^{-1} \cdot\left(\mathbf{X}-\mathbf{X}_{\text {mes }}\right)^{T}\right]^{1 / 2}
$$

where $\mathbf{C}$ is the covariance matrix of the noisy measurements. Ideally $\mathbf{C}$ would be obtained for each tissue type from measurements. For practical reasons we have approximated $\mathbf{C}$ with a single covariance matrix obtained from a noisy distribution of $\left[Z / Z_{w}, \rho_{\mathrm{e}} / \rho_{\mathrm{e}, \mathrm{w}}\right]$ from a $1 \mathrm{~cm}$ diameter ROI at centre of the dose calculation phantom. This is valid since in the soft tissue range $Z \in[6,8]$ the distribution of $\left[Z / Z_{w}, \rho_{\mathrm{e}} / \rho_{\mathrm{e}, \mathrm{w}}\right]$ is similar among tissue types. In the absence of noise $L_{M}$ reduces to the Euclidian distance.

\section{Dose calculations}

Following tissue segmentation from reconstructed 2D CT images of the dose calculation phantom a cylindrical geometry was created by stacking $172 \mathrm{~mm}$ thick identical $256 \times 256$ slices. 72 brachytherapy seeds were inserted in three planes following the $X Y$ pattern shown in Figure 5.3. The $Z$ spacing between seeds is $1.75 \mathrm{~cm}$ from centre to centre, with one seed plane positioned at the centre of the geometry. The geometry based on the dose calculation phantom containing breast tissues was implanted with ${ }^{103} \mathrm{Pd}$ seeds, model 2335, while the one containing prostate tissues was implanted with ${ }^{125}$ I seeds, model 2301 (both from Best Medical, Springfield, VA). Cylinders of water whose radii are the same as the dose calculation phantom were added at both ends of the geometry to provide $5 \mathrm{~cm}$ additional backscatter.

Using a GEANT4 ${ }^{30}$ based Monte Carlo dose calculation platform ${ }^{9}$ the dose to medium with transport in medium $D_{\mathrm{m}, \mathrm{m}}$ and the dose to water with transport in medium $D_{\mathrm{w}, \mathrm{m}}$ were calculated in each $0.53 \times 0.53 \times 2 \mathrm{~mm}^{3}$ voxel. $D_{\mathrm{w}, \mathrm{m}}$ was obtained using mass energy absorption coefficients and the photon energy fluence ${ }^{31}$. Statistical uncertainty varies with position in the phantom. The simulations were set up so the inner ring of inserts had statistical uncertainty below $1 \%$, calculated according to ${ }^{32}$. First, dose calculations were performed in a reference geometry where all exactly known tissue compositions and densities are correctly assigned from Tables 5.2 and 
5.3. The $D_{\mathrm{m}, \mathrm{m}}$ and $D_{\mathrm{w}, \mathrm{m}}$ distributions obtained from these calculations are our benchmarks. Thus in total 6 different geometries were employed for dose calculations: (i) the reference geometry, (ii) the TG43 geometry where the density is set to unity and tissue composition to water, (iii and iv) the SECT-3 and SECT-7 tissue geometries where the SECT segmentation schemes' output is used, (v) the DECT geometry where the output of the DECT segmentation scheme is used and (vi) the output of the segmentation scheme employing noisy DECT images. All resulting dose distributions were normalized to the reference or benchmark distribution from (i) prior to analysis. An ideal segmentation scheme would thus yield a normalized dose distribution having unity value at every voxel.

\section{RESULTS AND DISCUSSION}

\section{Imaging results}

The ability of our generic CT scanner simulations to generate realistic HU is shown in Figure 5.5 where we see the $\mu / \mu_{\mathrm{w}}=\mathrm{HU} / 1000+1$ difference between inserts from simulated images of the calibration phantom, which contains RMI tissue substitutes and the results of CT scans of an RMI phantom performed on a Picker PQ5000 CT scanner. Overall we see that ImaSim provides HU for RMI inserts that are representative of those measured with a clinical CT scanner. In the region of interest for this work (soft tissues, HU ranging from -200 to 200 ) we observe agreement within $\pm 5 \%$. Since our generic scanner model is not designed according to the PQ5000 specifications, perfect agreement is neither expected nor sought.

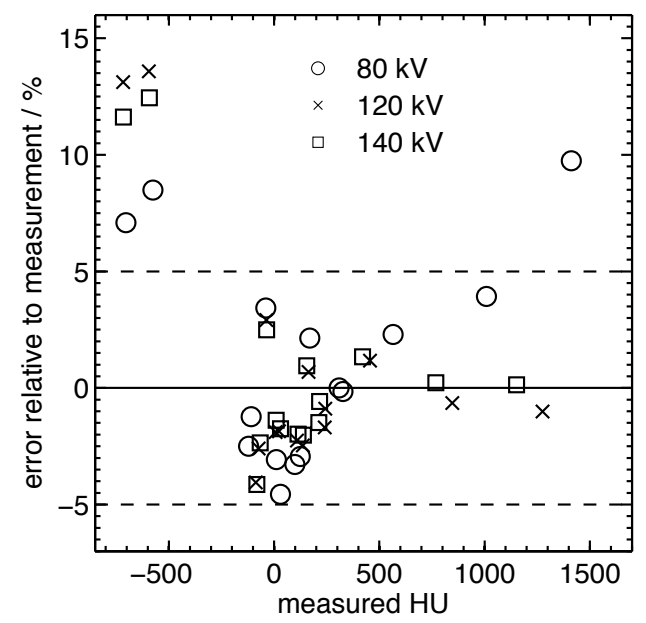

Figure 5.5: Difference (simulation - measurement) of $\mu / \mu_{w}$ values from our generic scanner simulations and from CT scans of a RMI phantom. 
The results of the dual energy algorithm applied to the pair of $80 \mathrm{kVp}$ and $140 \mathrm{kVp}$ images of the calibration phantom are presented in Figure 5.6. The algorithm results $\left[Z, \rho_{\mathrm{e}} / \rho_{\mathrm{e}, \mathrm{w}}\right]$ are compared to the theory $\left[Z_{\mathrm{eff}}, \rho_{\mathrm{e}} / \rho_{\mathrm{e}, \mathrm{w}}\right]$ in a scatter plot for each insert material. Since this is a noise-free simulation, no standard deviation is reported. It was determined that $n=3.31$ provides the best agreement between $Z$ and $Z_{\text {eff. }}$. This value is close to the often-used 3.5 recommended by Johns and Cunningham ${ }^{26}$ and agrees with the value of 3.3 obtained by Yang et al. ${ }^{16}$ where the same $Z_{\text {eff }}$ formulation is used. We see that the accuracy on $\rho_{\mathrm{e}} / \rho_{\mathrm{e}, \mathrm{w}}$ for soft tissues $\left(Z_{\text {eff }}<8\right)$ is well within $\pm 2 \%$, except for two lung mimicking inserts. The errors on the lung inserts are caused by a discrepancy between the expected HU and those given by ImaSim for low-density materials (Figure 5.5). A linear rescaling of ImaSim $\mathrm{HU}$ as proposed in Williamson et al. ${ }^{12}$ corrects the error. However, since lung is not a critical organ in brachytherapy we have not performed this rescaling in this study, and it is expected to have no significant influence on our results. Differences are observed between $Z$ and $Z_{\text {eff }}$ that vary linearly in the soft tissue range. $A$ fit of $Z_{\text {eff }}$ as a function of $Z$ yields the following relation:

$$
Z_{\text {cor }}=1.165 \times Z-1.188, Z \in[6,8]
$$

with $\mathrm{R}^{2}=0.995$ which can be used as an empirical correction. Errors on uncorrected $Z$ are limited to $\pm 6 \%$. These errors are similar to those of Bazalova et al. ${ }^{13}$ who reported errors of about $\pm 6 \%$ on $Z$ for RMI tissue substitutes. Goodsitt et al. ${ }^{33}$ used a rapid kVp switching clinical DECT scanner to measure $Z$ for RMI substitutes and also obtained results within $\pm 6 \%$.
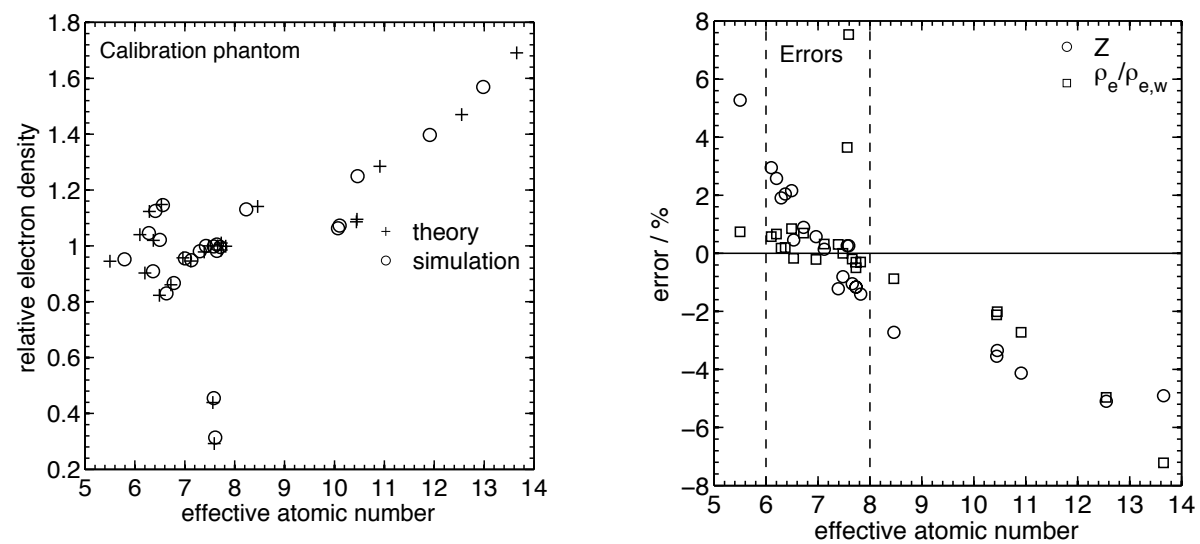

Figure 5.6: Left Scatter plot of the DECT algorithm results $\left[Z, \rho_{e} / \rho_{e, w}\right]$ and the theory $\left[Z_{e f f}, \rho_{e} / \rho_{e, w}\right]$. The abscissa is $Z_{\text {eff }}$ with $n=3.31$. Right The errors of the DECT algorithm relative to values from the theory, for $Z$ and $\rho_{e} / \rho_{e, w}$ plotted against $Z_{\text {eff. }}$.

The $\left[Z, \rho_{\mathrm{e}} / \rho_{\mathrm{e}, \mathrm{w}}\right]$ from each insert of both dose calculation phantoms (breast and prostate tissues) are compared to their theoretical values $(n=3.31)$ in Figure 5.7, with focus on soft tissues $Z \in[6,8]$. In addition, $\left[Z_{\text {cor }}, \rho_{\mathrm{e}} / \rho_{\mathrm{e}, \mathrm{w}}\right]$ is shown, obtained from a $Z$ 
image corrected from Equation 5.4. We see that for soft tissues the results indicate good agreement with the theoretical values for most tissues. The correction reduces errors on $Z_{\text {cor }}$ to $\pm 1 \%$, except for lung tissue $\left(Z_{\text {eff }}=7.60\right)$. This level of accuracy is within the 0.1 to 0.2 (in units of $Z$ ) requirement suggested by Mahnken et al. ${ }^{17}$ for soft tissue identification. Similar agreement is found for the relative electron density.
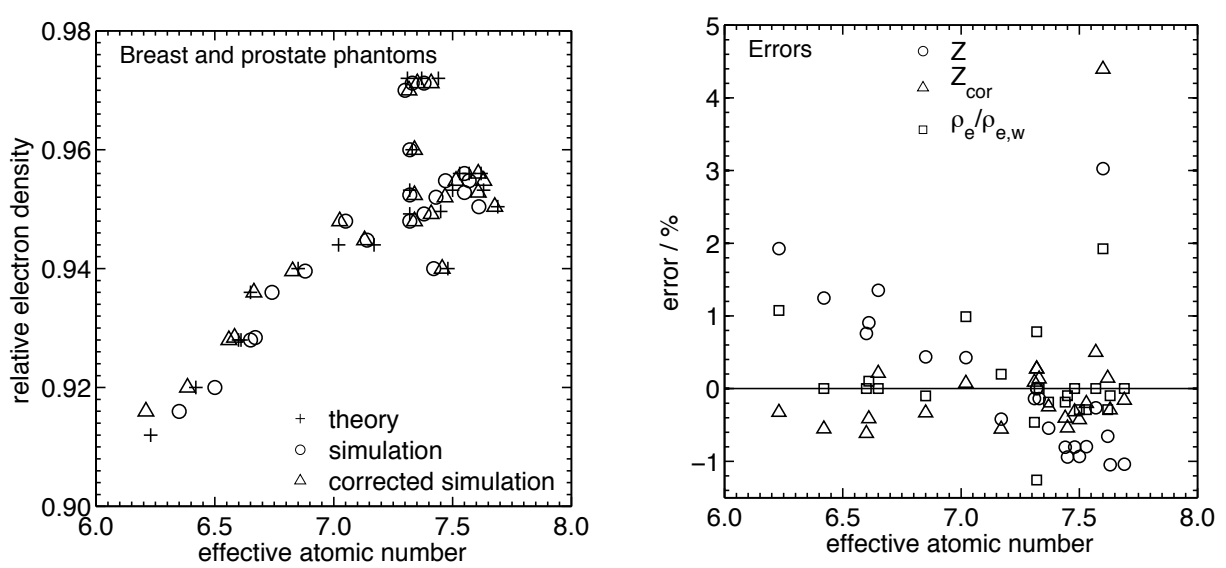

Figure 5.7: Left Scatter plot of the DECT results $\left[Z, \rho_{e} / \rho_{e, w}\right]$ and the theory $\left[Z_{e f f}, \rho_{e} / \rho_{e, w}\right]$ for tissue inserts in both dose calculation phantoms. The abscissa is $Z_{\text {eff }}$ with $n=3.31$. Also shown are corrected $Z$ values (Equation 5.4). Right The errors of the dual energy algorithm relative to values from the theory, for $Z$, corrected $Z$ and $\rho_{e} / \rho_{e, w}$ all plotted against $Z_{\text {eff. }}$.

\section{Segmentation and dose calculation results}

Breast phantom and ${ }^{103} \mathrm{Pd}$

Dose distributions calculated on segmented images of the breast dose calculation phantom with a ${ }^{103} \mathrm{Pd}$ implant and normalized to the reference are presented in Figure 5.8 , along with a color-coded representation of the material map. Even with DECT some inserts were assigned the wrong tissue type. This is usually the case for tissue types with very similar $Z_{\text {eff }}$ and $\rho_{\mathrm{e}} / \rho_{\mathrm{e}, \mathrm{w}}$ values. Note that the DECT segmentation was performed with the corrected atomic number map $Z_{\text {cor }}$ (Equation 5.4). These misassignments [inserts no. 10 (muscle hi Z instead of muscle mean Z), 13 and 20 (adipose hi $Z$ instead of breastA30G70)] do not cause important dose calculation errors since their tissue properties are similar (see Table 5.2). For the $D_{\mathrm{w}, \mathrm{m}}$ dose calculation, errors are limited to $\pm 3 \%$ when excluding the rib insert, while they are within $\pm 4 \%$ for $D_{\mathrm{m}, \mathrm{m}}$. Volume averaging artefacts around the rib insert cause some errors of $+10 \%$. It is easy to appreciate that DECT segmentation yields more accurate dose calculations than TG43 and SECT-3 segmentation. These two schemes perform poorly with ${ }^{103} \mathrm{Pd}$ for both scoring approaches with errors exceeding $20 \%$. 
82 |Chapter 5

A quantitative analysis of the data is provided in Figure 5.9 where we plot, for every tissue type, the mean value of the normalized dose within an insert as well as its standard deviation over the whole 3D insert. For most tissues the TG43 approach fails to approximate the correct dose deposition for either $D_{\mathrm{m}, \mathrm{m}}$ or $D_{\mathrm{w}, \mathrm{m}}$. The SECT-3 segmentation tends to reduce errors for $D_{\mathrm{w}, \mathrm{m}}$ but still falls outside $\pm 10 \%$ for some tissues.

Similar observations can be made for $D_{m, m}$, although SECT-3 actually performs worse than TG43 for some tissues. It is interesting to observe that in general, SECT-7 segmentation yields normalized dose distributions that are within $\pm 10 \%$ (considering the average doses in inserts) for both dose-scoring approaches. Rib is an exception to this; while the tissue type is correctly assigned by the segmentation scheme, the density is not $\left(1.51 \mathrm{gcm}^{-3}\right.$ from the segmentation vs. the correct $\left.1.41 \mathrm{gcm}^{-3}\right)$. This leads to a lower dose in the insert. The error on the SECT derived density is attributable to the rib insert $\left(\mathrm{HU}_{120 \mathrm{kVp}}=760\right)$ not falling on the $\mathrm{HU}-\rho$ curve established with the tissue mimicking inserts. This is because the tissue mimicking inserts do not have identical elemental composition as human tissues. A potential solution to this problem is the use of the stoichiometric calibration method ${ }^{34,35}$ to derive a human tissue specific $\mathrm{HU}$ $\rho$ curve.

When looking at the mean values of the normalized $D_{\mathrm{w}, \mathrm{m}}$ in Figure 5.9 we see that for DECT they are within $\pm 1 \%$ for all inserts except rib (+3\%). For $D_{m, m}$ the mean values range from $+3 \%$ to $-1 \%$. This is significantly better than SECT-7 which shows errors of up to $+9 \%$ for $D_{\mathrm{m}, \mathrm{m}}$ and $-6 \%$ for $D_{\mathrm{w}, \mathrm{m}}$. 


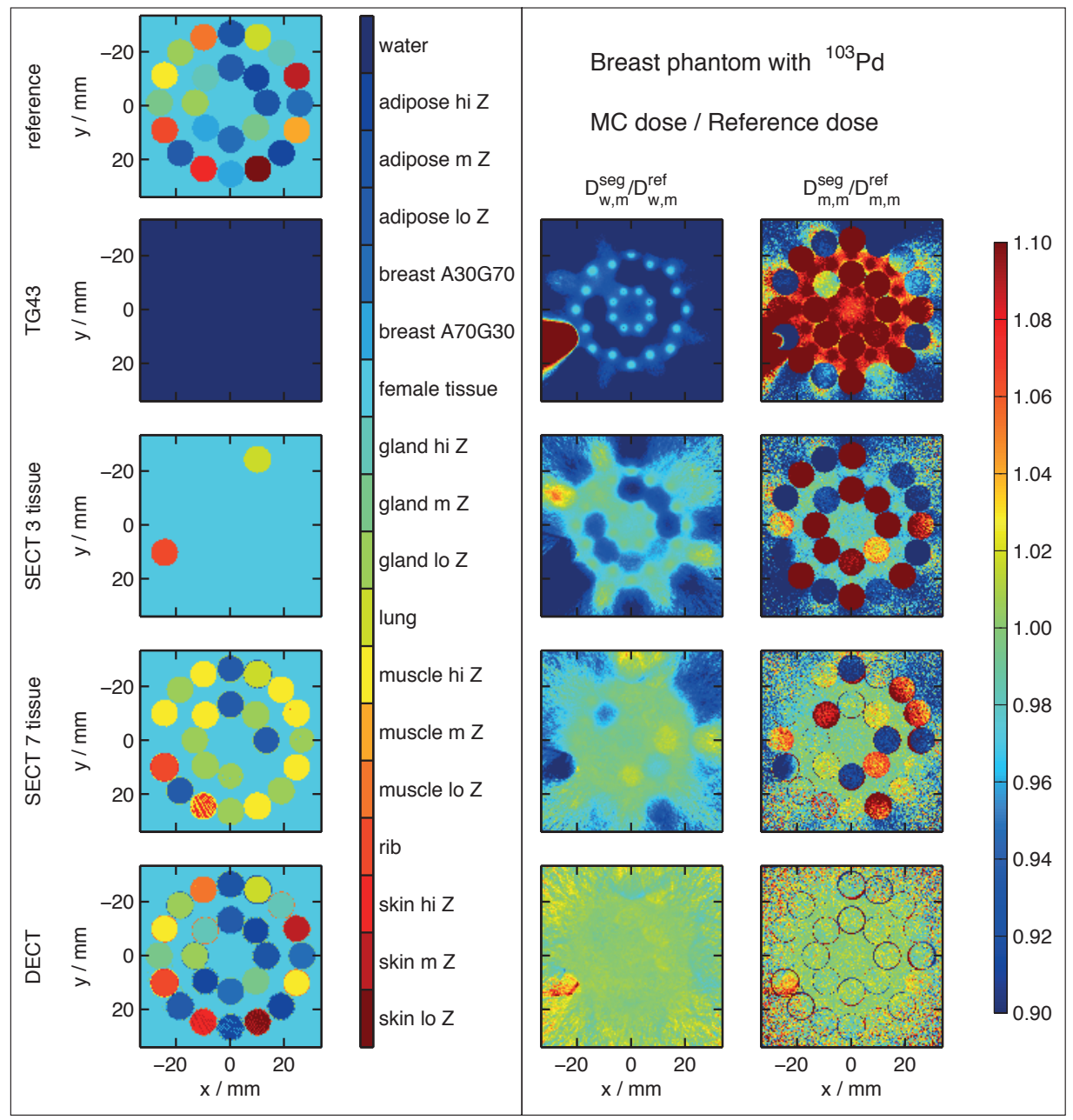

Figure 5.8: Left representation of the performance of various segmentation schemes in assigning tissue composition. The top segmentation is the reference. Right Normalized to reference $D_{w, m}$ and $D_{m, m}$ distributions for each segmentation scheme. The radiation source is ${ }^{103} \mathrm{Pd}$. 

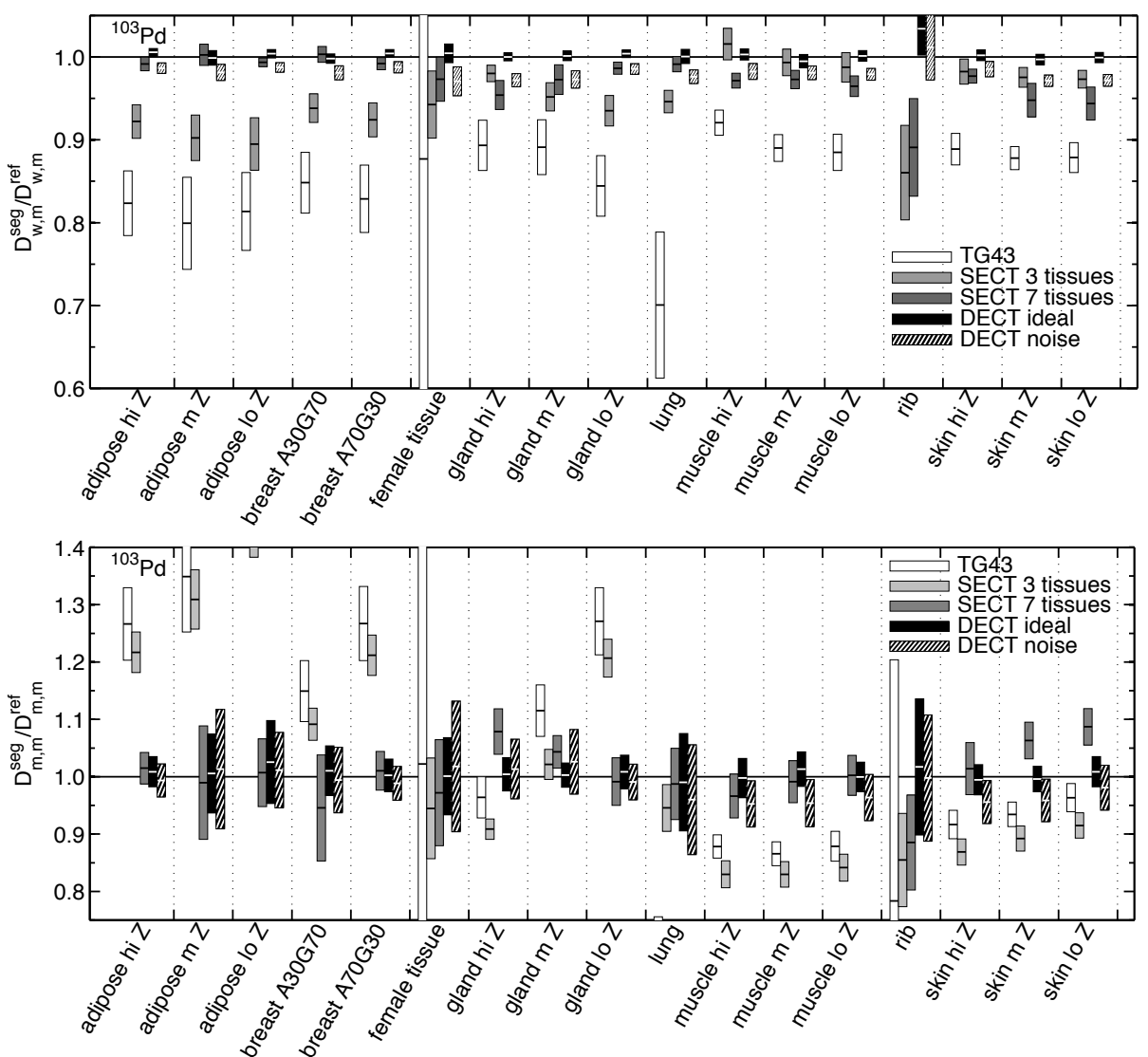

Figure 5.9: Quantitative analysis of dose calculation accuracy based on the segmentation schemes investigated in this work. The average dose normalized to the reference and the standard deviation is obtained in each insert of the breast dose calculation phantom. The central line of a bar indicates the average, with the top and bottom halves each indicating one standard deviation.

Prostate phantom and ${ }^{125}$,

Figures 5.10 and 5.11 present similar data for the prostate dose calculation phantom inserted with ${ }^{125} \mathrm{I}$ seeds. Similar behaviour as for ${ }^{103} \mathrm{Pd}$ in the breast phantom can be observed, although the magnitude of dose calculation errors is somewhat reduced with ${ }^{125}$ I because of its higher photon energy. This makes the dose calculation accuracy less sensitive to tissue misassignments. With DECT two inserts are misassigned [no. 7 (male soft tissue instead of prostate C) and no. 14 (prostate C instead of male soft tissue)]. These tissues are again very similar (see Table 5.3) and these errors do not impact the dose calculation accuracy. Some of the tissues from the breast dose calculation phantom are also found in the prostate dose calculation phantom, such as 
adipose tissue, muscle, rib and lung. These can be used to compare the behaviour of the two sources.

In this situation SECT-7 yields dose calculations whose accuracy is better than for ${ }^{103} \mathrm{Pd}$. When looking at the mean values of the normalized doses (Figure 5.11) we see that for $D_{\mathrm{w}, \mathrm{m}}$ they range from $+1 \%$ to $-2 \%$ for all inserts except rib (-7\%). For $D_{\mathrm{m}, \mathrm{m}}$ they range from $+3 \%$ to $-6 \%$ except rib and water $(-8 \%$ and $-7 \%)$.

In general $D_{\mathrm{w}, \mathrm{m}}$ appears to be less sensitive to tissue segmentation errors than $D_{\mathrm{m}, \mathrm{m}}$ for any scheme used. One possible explanation is that when scoring $D_{\mathrm{m}, \mathrm{m}}$ in a misassigned insert, both mass attenuation and mass energy absorption coefficients are erroneous. When scoring $D_{\mathrm{w}, \mathrm{m}}$ the mass energy absorption coefficient of water is used to score dose and only the error on the energy fluence remains, thus potentially reducing the amplitude of the difference with the reference situation.

The results from both dose calculation phantoms (breast and prostate) are valid for the particular geometries investigated. It is not obvious that these observations will hold for clinical patient geometries. The prostate is a relatively uniform tissue whose dimensions are larger than the inserts found in our phantom. The presence of calcifications in the tissue is also of importance. The breast is a complex arrangement of mammary gland embedded in adipose tissue where calcifications can also be found. As recent work suggests it is important to segment both gland and adipose tissues to accurately model the energy fluence in breast tissue ${ }^{36}$, there would be interest to evaluate the performance of DECT based segmentation. 
86 |Chapter 5

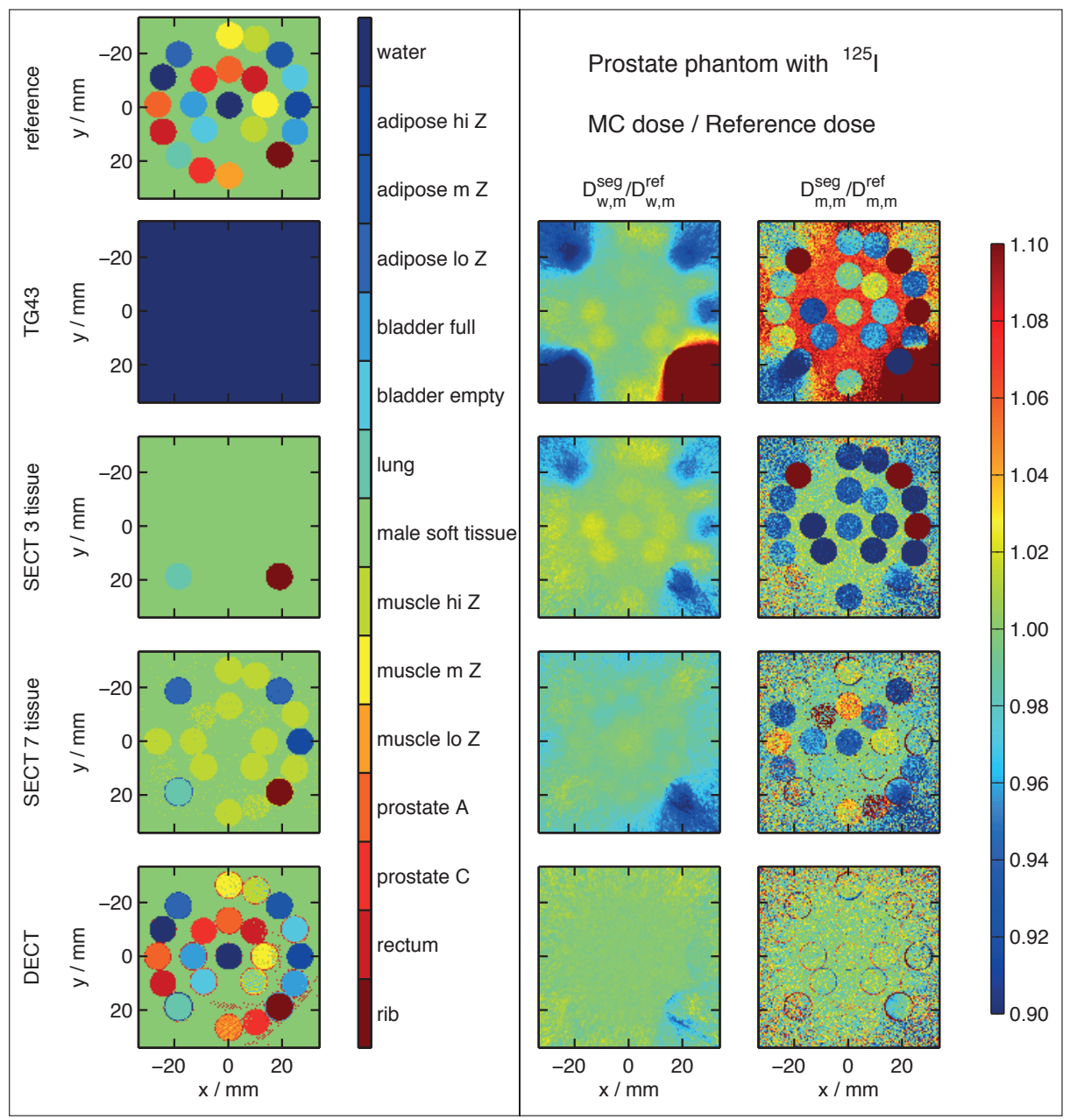

Figure 5.10: Left representation of the performance of various segmentation schemes in assigning tissue composition. The top segmentation is the reference. Right $D_{w, m}$ and $D_{m, m}$ distributions normalized to the reference for each segmentation scheme. The radiation source is ${ }^{125}$. 

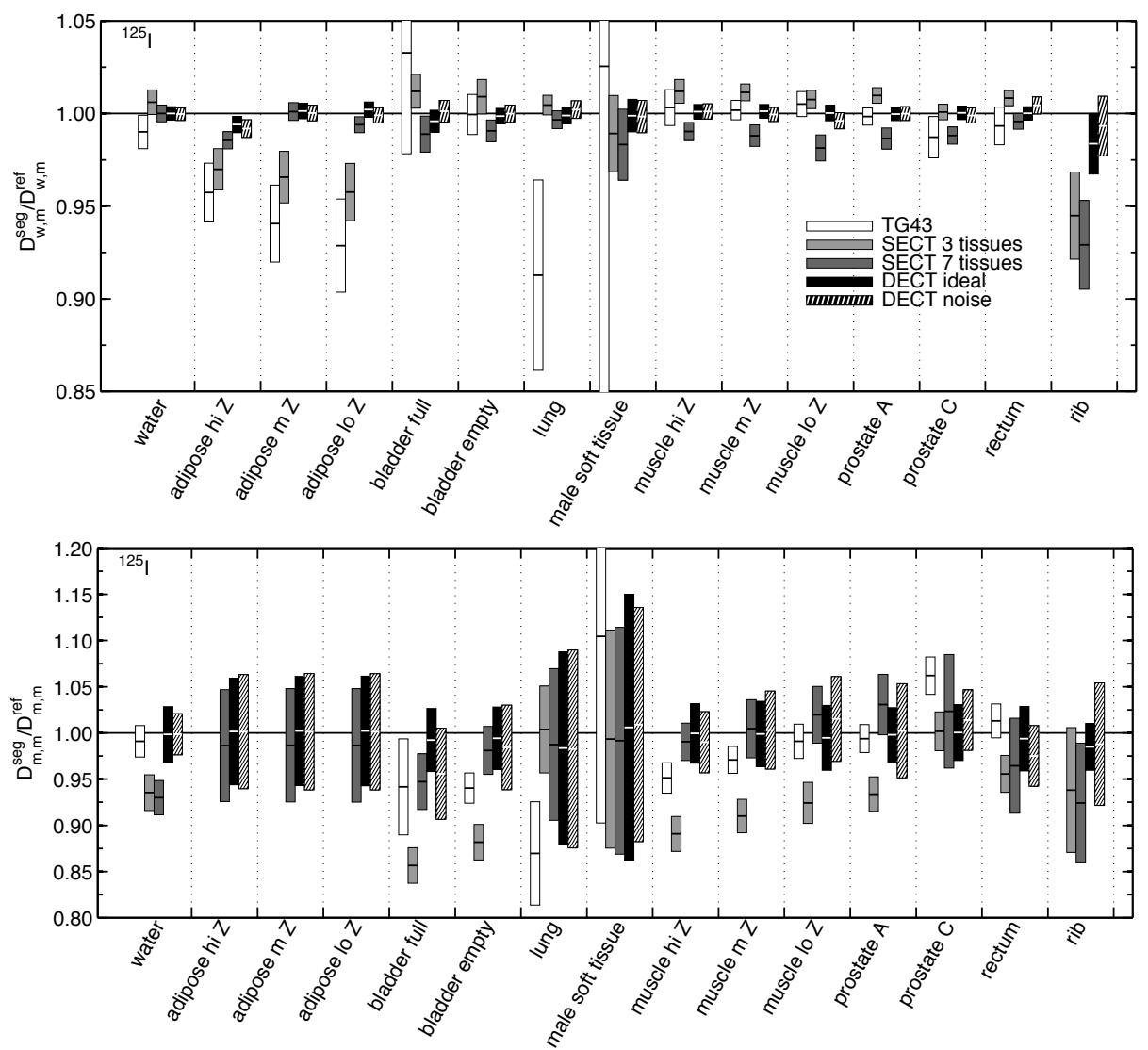

Figure 5.11: Quantitative analysis of dose calculation accuracy based on the segmentation schemes investigated in this work. The average dose normalized to reference and its standard deviation is obtained in each insert of the prostate dose calculation phantom. The central line of a bar indicates the average, with the top and bottom halves each indicating one standard deviation.

\section{Influence of noise}

The influence of image noise on the results is shown in Figure 5.12 where the distribution of $\left[Z, \rho_{\mathrm{e}} / \rho_{\mathrm{e}, \mathrm{w}}\right]$ is plotted for the tissues of the breast dose calculation phantom. We see that image noise creates clusters of $\left[Z, \rho_{\mathrm{e}} / \rho_{\mathrm{e}, \mathrm{w}}\right]$ values with a distinct shape. It is clear from Figure 5.12 that at most eight soft tissue categories can be segmented from this data out of the selected tissue range. Lung and bone are not shown in Figure 5.12 but can be segmented easily. The standard deviations of $\left[Z, \rho_{\mathrm{e}} / \rho_{\mathrm{e}, \mathrm{w}}\right]$ from noisy simulations are found in tabulated form in the online supplemental material accompanying this report. The average relative standard deviations is $2.9 \%$ for $Z$ and $1.2 \%$ for $\rho_{\mathrm{e}} / \rho_{\mathrm{e}, \mathrm{w}}$. This appears to be high when compared to the recent results of Goodsitt et al. ${ }^{33}$ who 
report relative standard deviations for $Z$ in the range of $0.3 \%$ to $0.8 \%$ for $\mathrm{RMI}$ tissue substitutes. The fact that some soft tissues cannot be differentiated does not necessarily mean that dose calculation accuracy is severely impacted, since some of those tissues may have very similar dosimetric properties.

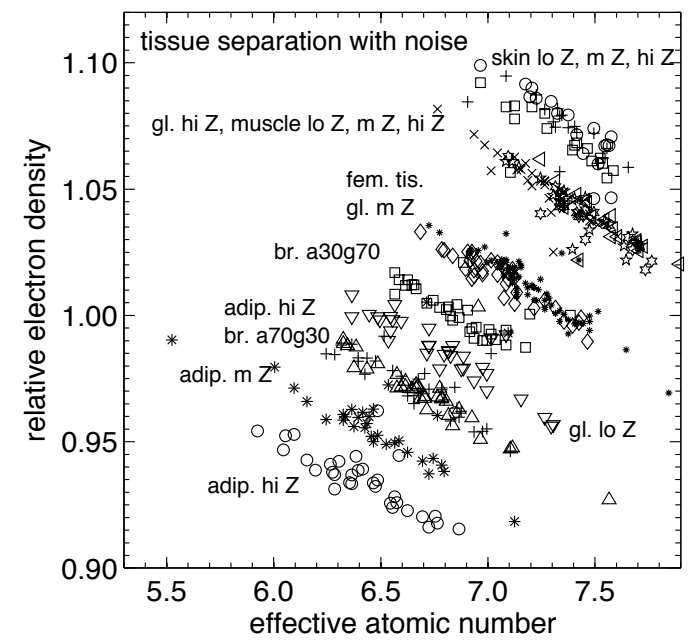

Figure 5.12: Influence of image noise on the results of our DECT algorithm illustrated by a $\left[Z, \rho_{e} / \rho_{e, w}\right]$ scatter plot. Several tissues fall within the same clusters.

Figures 5.9 and 5.11 present the results of DECT segmentation based on noisy data. Generally noise degrades the soft tissue dose calculation accuracy for both $D_{\mathrm{w}, \mathrm{m}}$ and $D_{\mathrm{m}, \mathrm{m}}$ by a few percent; the errors on the average doses range from to $+1 \%$ to $-3 \%$ for $D_{\mathrm{w}, \mathrm{m}}$ and $+3 \%$ to $-5 \%$ for $D_{\mathrm{m}, \mathrm{m}}$ in Figure 5.9 . Although no water insert is present in the breast dose calculation phantom, water was kept as an option in the segmentation. In the presence of noise, female soft tissue is partially misassigned to water. Since female soft tissue composes the bulk of the phantom, this misassignment has an impact. Removing water (a medium not expected to be present in brachytherapy procedures) from the segmentation procedure brings the abovementioned errors on the average doses down to $\pm 1 \%$ for $D_{\mathrm{w}, \mathrm{m}}$ and $+4 \%$ to $-3 \%$ for $D_{\mathrm{m}, \mathrm{m}}$.

A dose calculation was performed based on SECT-7 segmentation of the noisy $120 \mathrm{kVp}$ SECT image of the breast phantom. Differences between noisy and noise-free normalized dose distributions were inferior to $2 \%$, indicating that SECT-7 is robust with respect to noise, owing to the $50 \mathrm{HU}$ width of tissue bins.

\section{Significance of $\mathbf{Z}$}

We performed an idealized dose calculation for the breast phantom with ${ }^{103} \mathrm{Pd}$ where the chemical composition of all tissues was set to that of water, but the densities kept fixed to those of Table 5.2. When comparing this $\left(D_{\mathrm{w}, \mathrm{m}}\right)$ dose distribution to the one 
obtained with our ground truth (where $Z$ does vary) we observed dose differences of more than $\pm 20 \%$. This highlights the significance of knowing the chemical composition of each tissue.

To evaluate the significance of density assignment, we also performed dose calculations in fixed- $Z$ geometries where the densities were based on the SECT and DECT scans of the breast dose calculation phantom. Dose distributions were compared to the fixed- $Z$ idealized case described above. It was found that both SECT and DECT based dose distributions approximated the ground truth within $\pm 2 \%$, with the exception of the rib insert for SECT, which showed errors of up to $8 \%$ due to a $7 \%$ error on its density.

\section{CONCLUSIONS}

Images obtained from simulations of a SECT and DECT scanner were used to perform material segmentation. From noiseless DECT images, accuracy on $Z$ and $\rho_{\mathrm{e}} / \rho_{\mathrm{e}, \mathrm{w}}$ of human soft tissues was found to be within $\pm 1 \%$, following an empirical correction on $Z$ derived from simulated scans of a calibration phantom. Comparing ${ }^{103} \mathrm{Pd}$ and ${ }^{125} \mathrm{I}$ dose calculations performed in geometries segmented from noiseless SECT and DECT images showed that DECT based segmentation performs the best, with soft tissue dose calculation errors within $\pm 4 \%$ for ${ }^{103} \mathrm{Pd}$, the source most sensitive to tissue misassignments. This is an improvement upon SECT tissue segmentation using 7 tissue bins, which showed errors of up to $+9 \%$. The TG43 approach and SECT with 3 tissue bins fail to approximate the reference dose calculations. As these results are obtained with non-anthropomorphic phantoms, these conclusions might differ in clinical reality. Image noise was found to be an important factor which decreased the accuracy gains of DECT based tissue segmentation and warrants further investigation.

\section{ACKNOWLEDGEMENTS}

G.L. was supported by a PGSD2 scholarship from the Natural Sciences and Engineering Research Council of Canada (NSERC). P.G. was supported by a Kootstra Fellowship from CRISP, Centre for Research Innovation, Support and Policy of Maastricht University. B.R. was supported by a Marie Curie Reintegration Grant (No. PIRG05-GA-2009247878). This work was partially funded by Grant No. 017133 of the Canadian Cancer Society Research Institute. The authors would like to thank Georgi Nalbantov, Ruud van Stiphout and Mark Podesta for useful discussions. 


\section{APPENDIX. LINEAR ATTENUATION COEFFICIENT PARAMETERIZATION AND DECT ALGORITHM}

This work followed the approach of Bazalova et al. ${ }^{13,14}$ and relies on the following parameterization of the linear attenuation coefficient $\mu(E)$ on atomic number $Z$ and electron density $\rho_{\mathrm{e}}$

$$
\mu(E)=\rho_{\mathrm{e}}\left[Z^{4} F(E, Z)+G(E, Z)\right]
$$

for photon energy $E$, from Torikoshi et al. ${ }^{15}$ The first term models the photoelectric contribution and the second the combined Compton and Rayleigh scattering contributions to the linear attenuation coefficient. The functions $F(E, Z)$ and $G(E, Z)$ are quadratic fits to $\tau / \rho(E, Z) A Z^{-5} N_{\mathrm{A}}^{-1}$ and $\left[\sigma_{\mathrm{R}} / \rho(E, Z)+\sigma_{\mathrm{C}} / \rho(E, Z)\right] A Z^{-1} N_{\mathrm{A}}^{-1}$ respectively. $\tau / \rho, \sigma_{\mathrm{R}} / \rho$ and $\sigma_{\mathrm{C}} / \rho$ are the energy dependent photoelectric, Rayleigh and Compton mass attenuation coefficients of element $Z$ taken from the NIST XCOM database ${ }^{24}$. At every keV from $1 \mathrm{keV}$ to $140 \mathrm{keV}$, a fit was performed along $Z$.

If a compound is scanned with the spectrum $S_{i}(E)$ (where $i=1,2$ denote the low and high energy spectra used in DECT scanning) then its measured linear attenuation coefficient can be approximated by $\mu_{i}$

$$
\mu_{i}\left(\rho_{\mathrm{e}}, Z\right)=\rho_{\mathrm{e}} \int_{\text {spectrum }} \omega_{i}(E)\left[Z^{4} F(E, Z)+G(E, Z)\right] d E
$$

where

$$
\omega_{i}(E)=\frac{S_{i}(E) D(E)}{\int_{\text {spectrum }} S_{i}(E) D(E) d E}
$$

and $Z$ can be non-integer. By scanning with two different known photon spectra Equation 5.5 can be solved for $\rho_{\mathrm{e}}$ and $Z$. Attenuation data from $\mathrm{CT}$ is expressed in terms of Hounsfield units $\mathrm{HU}_{i}=1000\left(\mu_{i} / \mu_{i, \mathrm{w}}-1\right)$, where the subscript $\mathrm{w}$ denotes water. This means the inputs to our algorithm are $\tilde{\mu}_{i}=\mu_{i} / \mu_{i, \mathrm{w}}$ with $i=1,2$. The algorithm iteratively solves the following equation for $Z$ :

$$
Z^{4}-\frac{\tilde{\mu}_{2} W_{2} G_{1}-\tilde{\mu}_{1} W_{1} G_{2}}{\tilde{\mu}_{1} W_{1} F_{2}-\tilde{\mu}_{2} W_{2} F_{1}}=0
$$

where

$$
\begin{aligned}
& W_{i}=\rho_{\mathrm{e}, \mathrm{w}} \int_{\text {spectrum }} \omega_{i}(E)\left[Z_{\mathrm{w}}^{4} F\left(E, Z_{w}\right)+G\left(E, Z_{\mathrm{w}}\right)\right] d E, \\
& G_{i}=\int_{\text {spectrum }} \omega_{i}(E) G(E, Z) d E
\end{aligned}
$$

and

$$
F_{i}=\int_{\text {spectrum }} \omega_{i}(E) F(E, Z) d E
$$


To solve Equation $5.7 Z_{\mathrm{w}}$ is required to calculate $W_{i}$, which is used to convert $\tilde{\mu}_{i}$ to $\mu_{i}$ ( $\left.\mu_{i}=\tilde{\mu}_{i} W_{i}\right)$. In Bazalova et al. ${ }^{13} Z_{\mathrm{w}}=\left(0.112 \times 1^{3.5}+0.888 \times 8^{3.5}\right)^{1 / 3.5}=7.733$ is used. In this work we have chosen to solve Equation 5.7 once with $\tilde{\mu}_{i} W_{i}=0.112 \times \mu_{i}\left(\rho_{\mathrm{e}, \mathrm{H}}, 1\right)$ $+0.888 \times \mu_{i}\left(\rho_{\mathrm{e}, 0}, 8\right)$, yielding a $Z_{\mathrm{w}}$ which when used in Equation 5.8 yields a water linear attenuation coefficient that approximates the expected measurement from the system. Once Equation 5.7 has been solved for $Z$ the electron density $\rho_{\mathrm{e}}$ is obtained from

$$
\rho_{\mathrm{e}}=\frac{\tilde{\mu}_{i} W_{i}}{Z^{4} F_{i}+G_{i}}
$$

for either $i=1,2$. In Bazalova et al. $^{13}$ it is recommended to use spectra filtered by a water thickness equal to the radius of the phantom to account for beam hardening effects. We have adopted this approach in our algorithm.

\section{REFERENCES}

${ }^{1}$ R. Nath, L.L. Anderson, G. Luxton, K.A. Weaver, J.F. Williamson, and A.S. Meigooni, "Dosimetry of interstitial brachytherapy sources: recommendations of the AAPM Radiation Therapy Committee Task Group No. 43. American Association of Physicists in Medicine," Med Phys 22, 209-234 (1995).

${ }^{2}$ M.J. Rivard, et al., "Update of AAPM Task Group No. 43 Report: A revised AAPM protocol for brachytherapy dose calculations," Med Phys 31, 633-674 (2004).

${ }^{3}$ H. Afsharpour, J.-P. Pignol, B. Keller, J.-F. Carrier, B. Reniers, F. Verhaegen, and L. Beaulieu, "Influence of breast composition and interseed attenuation in dose calculations for postimplant assessment of permanent breast ${ }^{103}$ Pd seed implant," Phys Med Biol 55, 4547-4561 (2010).

${ }^{4}$ G.S. Burns and D.E. Raeside, "The accuracy of single-seed dose superposition for I- 125 implants," Med Phys 16, 627-631 (1989).

5J.F. Carrier, M. D'Amours, F. Verhaegen, B. Reniers, A.G. Martin, E. Vigneault, and L. Beaulieu, "Postimplant dosimetry using a Monte Carlo dose calculation engine: a new clinical standard," Int J Radiat Oncol Biol Phys 68, 1190-1198 (2007).

${ }^{6} \mathrm{O}$. Chibani and J.F. Williamson, "MCPI: a sub-minute Monte Carlo dose calculation engine for prostate implants," Med Phys 32, 3688-3698 (2005).

${ }^{7}$ R.M. Thomson, R.E. Taylor, and D.W. Rogers, "Monte Carlo dosimetry for $125 \mathrm{I}$ and 103Pd eye plaque brachytherapy," Med Phys 35, 5530-5543 (2008).

${ }^{8} \mathrm{C}$. Furstoss, et al., "Monte Carlo study of LDR seed dosimetry with an application in a clinical brachytherapy breast implant," Med Phys 36, 1848-1858 (2009).

${ }^{9} \mathrm{G}$. Landry, et al., "Sensitivity of low energy brachytherapy Monte Carlo dose calculations to uncertainties in human tissue composition," Med Phys 37, 5188-5198 (2010).

${ }^{10} \mathrm{~N}$. Papanikolaou, et al., "AAPM Report no. 85: Tissue inhomogeneity corrections for megavoltage photon beams. ," (2004).

${ }^{11} \mathrm{~F}$. Verhaegen and S. Devic, "Sensitivity study for CT image use in Monte Carlo treatment planning," Phys Med Biol 50, 937-946 (2005).

${ }^{12}$ J.F. Williamson, S. Li, S. Devic, B.R. Whiting, and F.A. Lerma, "On two-parameter models of photon cross sections: application to dual-energy CT imaging," Med Phys 33, 4115-4129 (2006). 
${ }^{13}$ M. Bazalova, J.F. Carrier, L. Beaulieu, and F. Verhaegen, "Dual-energy CT-based material extraction for tissue segmentation in Monte Carlo dose calculations," Phys Med Biol 53, 2439-2456 (2008).

${ }^{14}$ M. Bazalova, J.F. Carrier, L. Beaulieu, and F. Verhaegen, "Tissue segmentation in Monte Carlo treatment planning: a simulation study using dual-energy CT images," Radiother Oncol 86, 93-98 (2008).

${ }^{15} \mathrm{M}$. Torikoshi, et al., "Electron density measurement with dual-energy x-ray CT using synchrotron radiation," Phys Med Biol 48, 673-685 (2003).

${ }^{16}$ M. Yang, G. Virshup, J. Clayton, X.R. Zhu, R. Mohan, and L. Dong, "Theoretical variance analysis of single- and dual-energy computed tomography methods for calculating proton stopping power ratios of biological tissues," Phys Med Biol 55, 1343-1362 (2010).

${ }^{17}$ A.H. Mahnken, S. Stanzel, and B. Heismann, "Spectral rhoZ-projection method for characterization of body fluids in computed tomography: ex vivo experiments," Acad Radiol 16, $763-769$ (2009).

${ }^{18}$ B. Heismann and M. Balda, "Quantitative image-based spectral reconstruction for computed tomography," Med Phys 36, 4471-4485 (2009).

${ }^{19}$ B.J. Heismann, J. Leppert, and K. Stierstorfer, "Density and atomic number measurements with spectral x-ray attenuation method," J Appl Phys 94, 2073-2079 (2003).

${ }^{20}$ G. Landry, F. DeBlois, M. Bazalova, and F. Verhaegen. MO-D-303A-06: ImaSim, An Animated Tool for Teaching Imaging. 2009: AAPM.

${ }^{21}$ G. Poludniowski, G. Landry, F. DeBlois, P.M. Evans, and F. Verhaegen, "SpekCalc: a program to calculate photon spectra from tungsten anode x-ray tubes," Phys Med Biol 54, N433-438 (2009).

${ }^{22}$ G.G. Poludniowski, "Calculation of x-ray spectra emerging from an x-ray tube. Part II. X-ray production and filtration in x-ray targets," Med Phys 34, 2175-2186 (2007).

${ }^{23}$ G.G. Poludniowski and P.M. Evans, "Calculation of x-ray spectra emerging from an x-ray tube. Part I. electron penetration characteristics in x-ray targets," Med Phys 34, 2164-2174 (2007).

${ }^{24}$ M.J. Berger, J.H. Hubbell, S.M. Seltzer, J. Chang, and J.S. Coursey, XCOM: Photon Cross Sections Database. 2005, NIST.

${ }^{25} \mathrm{X}$. Pan, "Optimal noise control in and fast reconstruction of fan-beam computed tomography image," Med Phys 26, 689-697 (1999).

${ }^{26}$ H.E. Johns and J.R. Cunningham, The physics of radiology. 4th ed. 1983, Springfield, III., U.S.A.: Charles C. Thomas. xix, $796 \mathrm{p}$.

${ }^{27}$ ICRU, Photon, electron, proton, and neutron interaction data for body tissues. Report 46. 1992, Bethesda, MD.

${ }^{28}$ H.Q. Woodard and D.R. White, "The composition of body tissues," Br J Radiol 59, 1209-1218 (1986).

${ }^{29}$ ICRP, Basic anatomical and physiological data for use in radiological protection: reference values. Report 89. 2002, Oxford: Pergamon.

${ }^{30}$ S. Agostinelli, et al., "Geant4-a Simulation Toolkit," Nuclear Instruments \& Methods in Physics Research Section a-Accelerators Spectrometers Detectors and Associated Equipment 506, 250-303 (2003).

${ }^{31}$ G. Landry, B. Reniers, J.P. Pignol, L. Beaulieu, and F. Verhaegen, "The difference of scoring dose to water or tissues in Monte Carlo dose calculations for low energy brachytherapy photon sources," Medical Physics 38, 1526-1533 (2011).

${ }^{32}$ B.R. Walters, I. Kawrakow, and D.W. Rogers, "History by history statistical estimators in the BEAM code system," Med Phys 29, 2745-2752 (2002). 
${ }^{33}$ M.M. Goodsitt, E.G. Christodoulou, and S.C. Larson, "Accuracies of the synthesized monochromatic CT numbers and effective atomic numbers obtained with a rapid $\mathrm{kVp}$ switching dual energy CT scanner," Med Phys 38, 2222-2232 (2011).

${ }^{34} \mathrm{U}$. Schneider, E. Pedroni, and A. Lomax, "The calibration of CT Hounsfield units for radiotherapy treatment planning," Physics in medicine and biology 41, 111-124 (1996).

${ }^{35} \mathrm{~W}$. Schneider, T. Bortfeld, and W. Schlegel, "Correlation between CT numbers and tissue parameters needed for Monte Carlo simulations of clinical dose distributions," Phys Med Biol 45, 459-478 (2000).

${ }^{36} \mathrm{H}$. Afsharpour, J. Pignol, B. Keller, J. Carrier, B. Reniers, F. Verhaegen, and L. Beaulieu, SU-FF-T406: Toward a More Accurate Dose Calculation Technique Using a Semiautomatic Organ Contouring in Monte Carlo Post-Implant Assessment of Breast LDR Brachytherapy. Vol. 36. 2009: AAPM. 2615-2615. 



\section{CHAPTER}

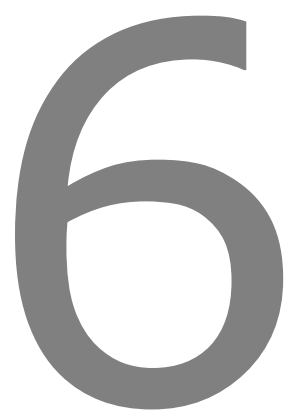

Extracting atomic numbers and electron densities from a dual source dual energy CT scanner: experiments and a simulation model

Guillaume Landry, Brigitte Reniers, Patrick Granton, Bart van Rooijen, Luc Beaulieu, Joachim E. Wildberger, Frank Verhaegen

Published in: Radiotherapy and Oncology 100(3):375-379, 2011. 


\section{ABSTRACT}

Purpose Dual energy CT (DECT) imaging can provide both the electron density $\rho_{\mathrm{e}}$ and effective atomic number $Z_{\text {eff, }}$ thus facilitating tissue type identification. This paper investigates the accuracy of a dual source DECT scanner by means of measurements and simulations. Previous simulation work suggested improved Monte Carlo dose calculation accuracy when compared to single energy CT for low energy photon brachytherapy, but lacked validation. As such, we aim to validate our DECT simulation model in this work.

Materials and Methods A cylindrical phantom containing tissue mimicking inserts was scanned with a second generation dual source scanner (SOMATOM Definition FLASH) to obtain $Z_{\text {eff }}$ and $\rho_{\mathrm{e}}$. A model of the scanner was designed in ImaSim, a CT simulation program, and was used to simulate the experiment.

Results Accuracy of measured $Z_{\text {eff }}$ (labeled $Z$ ) was found to vary from $-10 \%$ to $10 \%$ from low to high $Z$ tissue substitutes while the accuracy on $\rho_{\mathrm{e}}$ from DECT was about $4 \%$. Our simulation reproduced the experiments within $\pm 5 \%$ for both $Z$ and $\rho_{\mathrm{e}}$.

Conclusions A clinical DECT scanner was able to extract $Z$ and $\rho_{\mathrm{e}}$ of tissue substitutes. Our simulation tool replicates the experiments within a reasonable accuracy. 


\section{INTRODUCTION}

A computed tomography (CT) scan provides a measurement of the photon linear attenuation coefficients $\mu$ of the scanned object, often expressed as Hounsfield Units (HU). As the $\mathrm{HU}$ of a given material varies with photon energy, the measured values are specific to the tube potential and filtration used and may not be directly employed to perform dose calculations in another photon energy range. Furthermore, the attenuation coefficient is a function of both medium density and elemental composition. Thus, there can be several materials with the same HU having different densities and elemental compositions, complicating the use of CT for identifying material properties in applications such as dose calculation ${ }^{1}$.

By measuring the linear attenuation coefficient at two different tube potentials, dual energy CT (DECT) provides a means to decompose $\mu$ in two components, namely the electron density $\rho_{\mathrm{e}}$ and effective atomic number $Z$ (we call $Z$ the value provided by DECT and $Z_{\text {eff }}$ the expected quantity), thus facilitating tissue type identification. The potential benefits of using DECT in radiotherapy have been investigated in the context of brachytherapy dose calculations ${ }^{2}$, proton stopping power ratio estimation ${ }^{3,4}$ as well as $\mathrm{kV}$ and $\mathrm{MV}$ photon dose calculations $\mathrm{s}^{5,6}$. Other studies investigated optimal filtration of the high and low kVp photon spectra for image quality improvement ${ }^{7,8}$. Several of these studies are based on simulations rather than direct measurements, as DECT scanners are only now becoming clinically available. Goodsitt et al. have recently reported on the accuracy of experimentally derived atomic numbers provided by a DECT scanner based on the single source, rapid kVp switching design ${ }^{9}$.

In this work a second-generation dual source DECT scanner is investigated. In this design, two pairs of $x$-ray tube and detector array rotate around the patient, simultaneously acquiring a high and low kVp image. We report on the accuracy of $Z$ and $\rho_{\mathrm{e}}$ obtained from measurements of a standard electron density calibration phantom using the algorithm of Bazalova et al. ${ }^{5,6}$, adapted from Torikoshi et al. ${ }^{10}$ In addition to measurements, a simulation model of the DECT scanner has been designed. By comparing results from simulation and measurements we aim to validate the use of the simulation tool for DECT research.

A recent simulation study by our group explored the use of DECT as an alternative to SECT based dosimetry in low dose rate brachytherapy dose calculations ${ }^{11}$, which are highly sensitive to tissue composition assignment ${ }^{12}$. Calculated dose distributions from simulated CT images segmented into tissue composition and density using a SECT technique compared to a DECT technique proved more accurate using DECT. As our simulations $^{11}$ supported the use of DECT imaging for low energy photon dose calculations, validating our findings using a scanner readily available to the clinic is the focus of this manuscript. 


\section{MATERIALS AND METHODS}

\section{Experiment}

A cylindrical RMI 465 phantom (Gammex Inc., Middleton, WI) was scanned at a second generation dual source CT scanner (SOMATOM Definition FLASH, Siemens Healthcare, Forchheim, Germany) operated in dual energy mode. The scanner has two $x$-ray tube and detector array pairs, their respective fields of view being $34 \mathrm{~cm}$ and 50 $\mathrm{cm}^{13}$. Tube potentials were $80 \mathrm{kVp}$ and $140 \mathrm{kVp}$, with additional Sn filtration for the high $\mathrm{kVp}(140 \mathrm{kVp} / \mathrm{Sn})$. Exposures of 900 and $348 \mathrm{mAs}$ were used to minimize noise. Images were reconstructed at the scanner with a very smooth filter (B10f), again to reduce noise. The geometry of the phantom inserts is shown in Figure 6.1(a) and material properties are listed in Table 6.1. The effective atomic number $Z_{\text {eff }}$ reported in Table 6.1 is calculated according to the following equation:

$$
Z_{\text {eff }}=\left(\frac{\sum_{i} f_{i} \rho_{\mathrm{e}, i} Z_{i}^{3.3}}{\sum_{i} f_{i} \rho_{\mathrm{e}, i}}\right)^{1 / 3.3}
$$

where $f_{i}$ is the mass fraction of element $i$. In this paper we call $Z_{\text {eff }}$ the quantity derived from Equation 6.1 and $Z$ the quantity provided by the DECT algorithm.

Table 6.1: Properties of the inserts of the RMI 465 phantom. The insert numbers correspond to the numbering in Figure 6.1. $Z_{\text {eff }}$ values are derived from the compositions of Watanabe et al. ${ }^{21}$ using Equation 6.1. HU obtained from measurements at the DECT scanner are listed.

\begin{tabular}{|c|c|c|c|c|c|c|}
\hline $\begin{array}{l}\text { Insert } \\
\text { numbers }\end{array}$ & Material & $\begin{array}{c}\rho \\
\left(\mathrm{g} \mathrm{cm}^{-3}\right)\end{array}$ & $\rho_{\mathrm{e}} / \rho_{\mathrm{e}, \mathrm{w}}$ & $Z_{\text {eff }}$ & $\begin{array}{c}\mathrm{HU} \\
80 \mathrm{kVp}\end{array}$ & $\begin{array}{c}\mathrm{HU} \\
140 \mathrm{kVp} / \mathrm{Sn}\end{array}$ \\
\hline $1,3,5,6$ & Solid water & 1.015 & 1.000 & 7.73 & 6 & -4 \\
\hline 2 & Brain & 1.045 & 1.039 & 7.20 & -54 & -38 \\
\hline 4 & CB2-50\% & 1.560 & 1.473 & 12.54 & 1068 & 660 \\
\hline 7,15 & Acrylic & 1.180 & 1.147 & 6.53 & 107 & 146 \\
\hline 8 & Breast & 0.990 & 0.980 & 6.93 & -54 & -38 \\
\hline 9 & AP6 & 0.920 & 0.895 & 6.34 & -139 & -72 \\
\hline 10 & LN-300 & 0.300 & 0.292 & 7.59 & -718 & -720 \\
\hline 11 & SB3 & 1.840 & 1.707 & 13.69 & 1673 & 1006 \\
\hline 12 & CB2-30\% & 1.340 & 1.285 & 10.89 & 605 & 390 \\
\hline 13 & IB1 & 1.120 & 1.081 & 9.68 & 297 & 161 \\
\hline 14 & CB4 & 1.150 & 1.116 & 6.37 & 83 & 116 \\
\hline 16 & Liver & 1.080 & 1.050 & 7.73 & 89 & 85 \\
\hline 17 & B200 & 1.145 & 1.099 & 12.08 & 319 & 181 \\
\hline 18 & CB2-10\% & 1.170 & 1.142 & 8.46 & 202 & 163 \\
\hline 19 & LN-450 & 0.450 & 0.438 & 7.59 & -574 & -580 \\
\hline 20 & Polyethylene & 0.920 & 0.895 & 5.50 & -113 & -69 \\
\hline
\end{tabular}



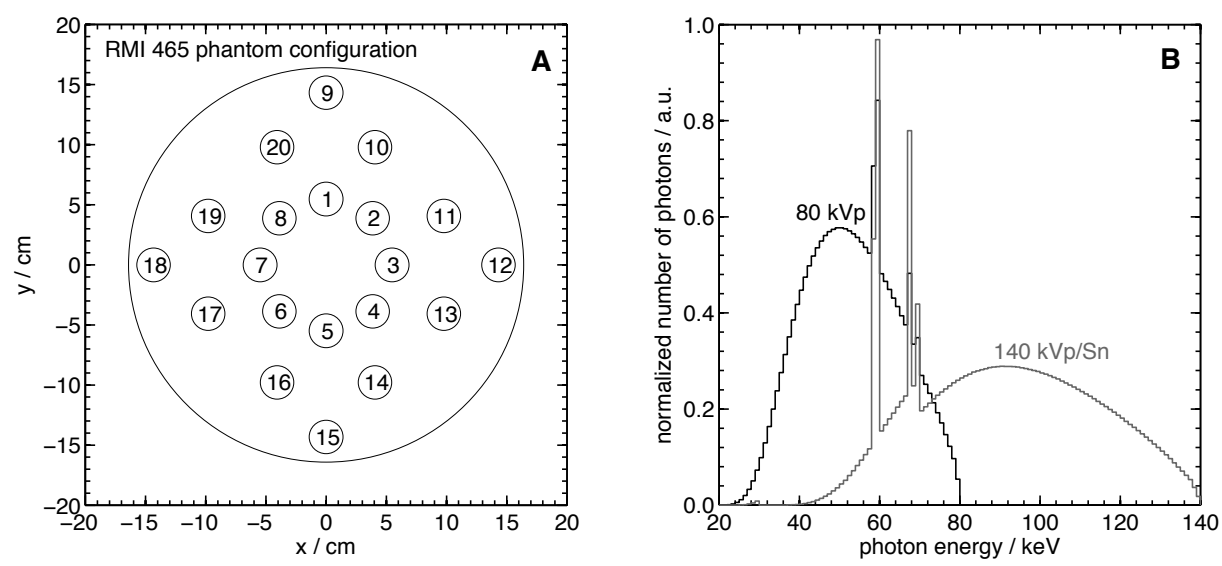

Figure 6.1: (A) Configuration of the cylindrical RMI 465 phantom used in the experiments and simulations. The correspondence between insert number and material type can be found in Table 6.1. (B) CT scanner photon spectra used in the DECT algorithm and ImaSim simulations.

From the $\mathrm{CT}$ images the atomic number $Z$ and the electron density relative to water, $\rho_{\mathrm{e}} / \rho_{\mathrm{e}, \mathrm{w}}$, were obtained ${ }^{5,6}$. The CT scanner spectra required by the algorithm are shown in Figure 6.1(b) and were generated with SpekCalc ${ }^{14-16}$ by adjusting manufacturer nominal tube filtration to match measured half value layers (HVLs). The HVL of the scanner was measured at 80,100, 120 and $140 \mathrm{kVp}$ using a localization technique ${ }^{17}$. The $\mathrm{CT}$ scanner $\mathrm{Gd}_{2} \mathrm{O}_{2} \mathrm{~S}$ detector response was also accounted for in the analysis $^{18}$. Prior to analysis, three slices (thickness $=3 \mathrm{~mm}$ ) were averaged and the $512 \times 512$ image matrices were rebinned to $256 \times 256$ to reduce noise and reduce calculation times.

\section{Simulation}

We simulated the experiment using ImaSim $^{19}$, a program using SpekCalc, which calculates $x$-ray projection images using ray tracing for a fan beam CT geometry. The attenuation coefficients of the tissue mimicking inserts were generated with the NIST XCOM database ${ }^{20}$ based on the compositions found in Watanabe et al. ${ }^{21}$. The bowtie filters of the CT scanner were not modelled. The same spectra and detector response as mentioned above were used and images were reconstructed with a Shepp Logan filter ${ }^{22}$. A cupping artefact correction was applied; the correction results in uniform $\mathrm{HU}$ values when applied to projections of a uniform water cylinder of the same radius as the cylindrical phantom. To match attenuation coefficients generated from ImaSim simulations to those calculated using the spectrum and detector response of the CT scanner the following equation was used: 


$$
\frac{\mu}{\mu_{\text {water }}}=\frac{\mathrm{HU}}{A}+B
$$

where $A$ and $B$ are obtained by fitting ${ }^{2}$. The parameter $A$ took the value 967.7 at 80 $\mathrm{kVp}$ and 986.4 at $140 \mathrm{kVp} / \mathrm{Sn}$ while $B$ was unity for both spectra.

\section{RESULTS}

The measured HVL are found in Table 6.2 and are compared to the nominal values provided by the CT scanner manufacturer. As it was not possible to operate the scanner in a parked position with the Sn filter in place, the HVL for the $140 \mathrm{kVp} / \mathrm{Sn}$ beam could not be measured. It was necessary to add $0.15 \mathrm{~mm} \mathrm{Ti}$ in SpekCalc to the filtration reported by the manufacturer to match the modelled and measured HVL.

Table 6.2: HVL measured at the DECT scanner compared to manufacturer provided values. The manufacturer's stated filtration was modified by adding $0.15 \mathrm{~mm}$ Ti to obtain the SpekCalc model HVL.

\begin{tabular}{llll}
\hline $\mathrm{kVp}$ & Measured HVL & $\begin{array}{l}\text { Nominal } \\
(\mathrm{mm} \mathrm{Al})\end{array}$ & Model \\
\hline 80 & 6.1 & 5.8 & 6.2 \\
100 & 7.4 & 7.1 & 7.5 \\
120 & 8.5 & 8.1 & 8.6 \\
140 & 9.5 & 9.1 & 9.4 \\
$140 / \mathrm{Sn}$ & - & 13.7 & 13.8 \\
\hline
\end{tabular}

Figure 6.2 presents the discrepancies between the attenuation coefficients obtained from scans of the RMI phantom at $80 \mathrm{kVp}$ and $140 \mathrm{kVp} / \mathrm{Sn}$ and those obtained from ImaSim with the same photon spectra. The measured $\mu$ are obtained from Equation 6.2 using $A=1000$ and $B=1$ while those from ImaSim use the $A$ and $B$ obtained from the fitting procedure. The $\mathrm{HU}$ used in Equation 6.2 are the averages from a circular ROI positioned at each insert. The standard deviation averaged over all inserts was $18 \mathrm{HU}$ at $80 \mathrm{kVp}$ and $13 \mathrm{HU}$ at $140 \mathrm{kVp} / \mathrm{Sn}$. 


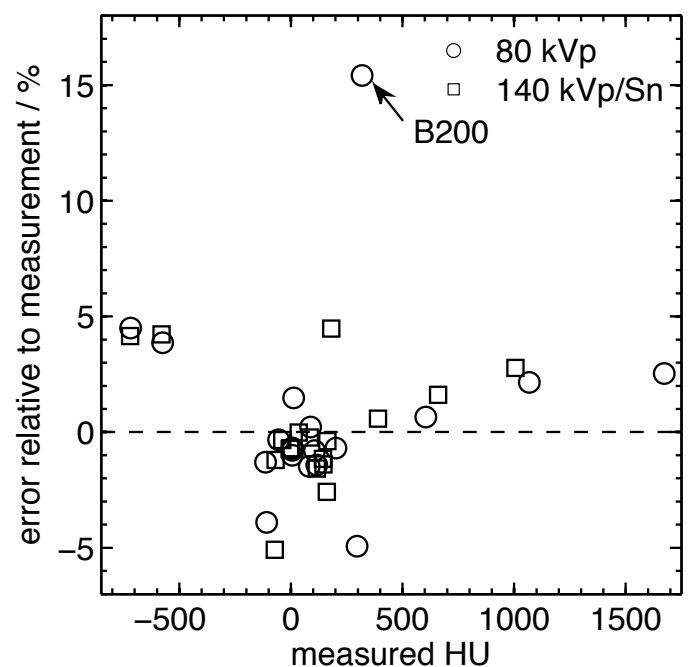

Figure 6.2: Differences of $\mu / \mu_{\text {water }}$ from ImaSim relative to the values measured at the CT scanner (ImaSim measured)/measured at two different $k V p$ for the RMI 465 phantom. Each data point represents the error on the average value measured in a circular ROI centred on each insert.

Representations of the $Z$ and $\rho_{\mathrm{e}} / \rho_{\mathrm{e}, \mathrm{w}}$ images obtained from the DECT algorithm applied to measured and simulated pairs of high and low $\mathrm{kVp}$ images are shown in Figure 6.3. The difference between the ROI averaged $Z$ from Figure 6.3 and the $Z_{\text {eff }}$ of Table 6.1 is plotted in Figure 6.4(a) for each insert, for both simulation and measurement. The average measured standard deviation on the $Z$ of an insert is $3 \%$. Figure 6.4(b) plots the difference between $\rho_{\mathrm{e}} / \rho_{\mathrm{e}, \mathrm{w}}$ from the DECT algorithm and the theoretical values from Table 6.1. The average measured standard deviation on $\rho_{\mathrm{e}} / \rho_{\mathrm{e}, \mathrm{w}}$ obtained from the DECT algorithm is $1 \%$. In addition to the results from the DECT algorithm, Figure 6.4(b) also shows the accuracy on the $\rho_{\mathrm{e}} / \rho_{\mathrm{e}, \mathrm{w}}$ obtained from a standard SECT based $\mathrm{HU}-\rho_{\mathrm{e}}$ calibration and the measured $140 \mathrm{kVp} / \mathrm{Sn}$ image. Figure 6.4(c) plots the ratio of results from simulations and measurements for both $Z$ and $\rho_{\mathrm{e}} / \rho_{\mathrm{e}, \mathrm{w}}$ for each insert in the RMI phantom. 

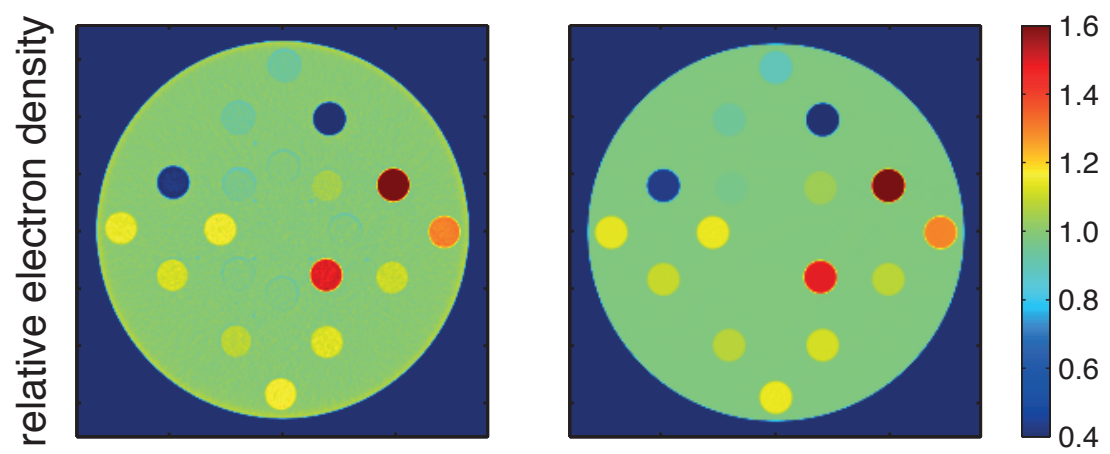

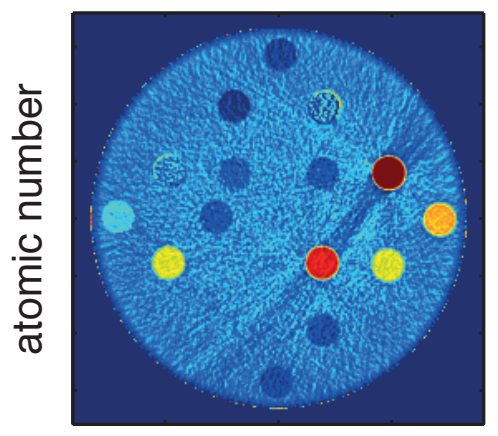

measured

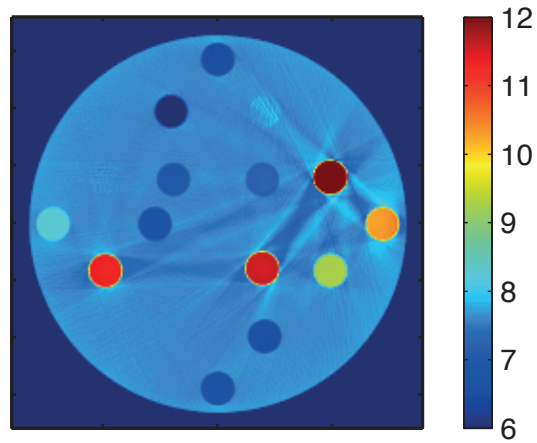

simulated

Figure 6.3: Colour representation of the atomic number $Z$ and relative electron density $\rho_{e} / \rho_{e, w}$ obtained from measurements at the CT scanner (left) and from ImaSim (right). Note that ImaSim simulations do not contain quantum noise.
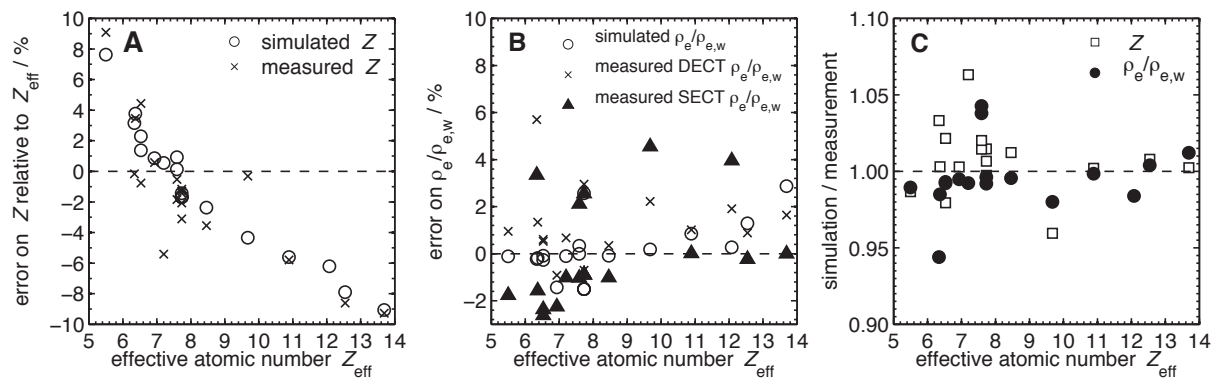

Figure 6.4: (A) Accuracy of atomic number from the DECT algorithm $\left(Z-Z_{\text {eff }}\right) / Z_{\text {eff }}$ for the measurement and simulation. The error on the measured $Z$ for the B200 insert $\left(Z_{\text {eff }}=12.08\right)$ is $-19 \%$ (B) Accuracy for $\rho_{e} / \rho_{e, w}$. In addition to the DECT measured and simulated electron density, the $\rho_{e} / \rho_{e, w}$ from the SECT calibration based on measurements is also shown. (C) Ratio of simulated and measured $Z$ and $\rho_{e} / \rho_{e, w}$ quantifying the accuracy of the simulation. 


\section{DISCUSSION}

In general, the relative photon linear attenuation coefficients $\mu / \mu_{\text {water }}$ generated from ImaSim using Equation 6.2, following beam hardening correction, agree well with those measured at the scanner. We observe in Figure 6.2 that except for the B200 insert scanned at $140 \mathrm{kVp} / \mathrm{Sn}$, all values agree within $\pm 5 \%$. The error on the B200 insert, which mimics bone mineral, is $15 \%$ at $80 \mathrm{kVp}$ and $4 \%$ at $140 \mathrm{kVp} / \mathrm{Sn}$, indicating that we did not properly simulate this insert. The cause for this discrepancy is not obvious to us but a potential explanation would be a mismatch between the composition used in the simulation and that of the actual insert. The B200 insert is also the only one containing a high amount of fluorine $(17.66 \%)^{21}$.

Figure 6.3 shows that ImaSim reproduces the $Z$ and $\rho_{\mathrm{e}} / \rho_{\mathrm{e}, \mathrm{w}}$ from the experiment; most inserts show similar values in the measured and simulated maps. From the measured data we can observe that the $Z$ image contains more noise than the $\rho_{\mathrm{e}} / \rho_{\mathrm{e}, \mathrm{w}}$ image. The noise levels on the $Z$ image are about 3\% while they are around $1 \%$ for the $\rho_{\mathrm{e}} / \rho_{\mathrm{e}, \mathrm{w}}$ image. As quantum noise was not included in the simulation, the simulated images appear smooth. The atomic number of the B200 insert obtained from the measurement (lower left quadrant of phantom, insert 17) is not reproduced correctly by the simulation. The lung inserts are also of interest; their $Z_{\text {eff }}=7.59$ is close to solid water's $\left(Z_{\text {eff }}=7.73\right)$. On the simulated $Z$ image, the $L N 450$ insert $\left(Z_{\text {LN450 }}=7.66\right)$ is barely distinguishable from the solid water background while in the measured image $\left(Z_{\mathrm{LN} 450}=7.55\right)$ it is easily recognized. This is because the noise pattern in the measured image is different in the lung insert than in the solid water background, probably due to the low density in the insert. There is also a more significant volume averaging effect at the edge of the insert in the measurement.

We see in figure 6.4(a) that the difference between the measured $Z$ and $Z_{\text {eff }}$ ranges from $+10 \%$ at low $Z_{\text {eff }}$ to $-10 \%$ at high $Z_{\text {eff. }}$ While these differences are significant, they are well reproduced by the simulations, which show a similar trend. Three inserts show measured $Z$ deviating from the trend observed in figure 4a; Brain, IB1, and B200. We see in figure $4 c$ that the ratio simulated/measured takes the values $1.06,0.96$ and 1.17 for these inserts.

The $\rho_{\mathrm{e}} / \rho_{\mathrm{e}, \mathrm{w}}$ obtained from DECT measurements appear to be systematically higher than the values of Table 6.1. This could potentially be attributed to an erroneous estimation of the scanner spectra or to the way the cupping correction is applied by the scanner software. This is in contrast with the results from the simulation, which show $\pm 2 \%$ accuracy. The relative electron densities from the standard SECT calibration show similar accuracy as those from DECT.

Figure 6.4 (c) shows that for the majority of inserts, both $Z$ and $\rho_{\mathrm{e}} / \rho_{\mathrm{e}, \mathrm{w}}$ obtained by the simulations agree with the measured values within $\pm 5 \%$. In addition to the three inserts mentioned above whose simulated $Z$ disagreed with measurements, the AP6 insert has a 0.91 value for the $\rho_{\mathrm{e}} / \rho_{\mathrm{e}, \mathrm{w}}$ simulated/measured ratio. These results show that ImaSim can be used to simulate the behaviour of a DECT scanner with reasonable accuracy. The simulation model can thus be used to investigate DECT research topics such as Z-map based contouring. The added information of DECT might facilitate tasks 
such as atlas-based automatic contouring and organ recognition. The model might also be used to optimize scanner geometries and scan protocols. Although noise has not been included in the simulations, it is relatively easy to add noise to the detector signals to generate noisy DECT results. To obtain the relatively low standard deviation of $3 \%$ on $Z$ measurements, it was necessary to use a relatively high $\mathrm{mAs}$ setting for our scans. The patient dose of such scans needs to be evaluated in the context of radiotherapy, where patients receive large radiation dose as part of their treatment. Such an evaluation is beyond the scope of this paper. A shortcoming of our model is the lack of bowtie filter modelling.

In brachytherapy seed implants, outcome and toxicity are generally correlated with post implant CT dosimetry ${ }^{23-25}$. This is currently performed using the TG-43 proto$\operatorname{col}^{26,27}$, whose accuracy has been challenged ${ }^{28-30}$. By using accurate Monte Carlo dose calculation techniques, improved dose-outcome relationships could possibly be derived. In our previous work $^{31}$, we quantified Monte Carlo dose calculation accuracy gains from DECT for ${ }^{125} \mathrm{I}$ and ${ }^{103} \mathrm{Pd}$ sources. We performed dose calculations based on simulated SECT and DECT images of a virtual phantom containing a series of human tissues of interest for prostate and breast seed implants. We showed significantly improved dose calculation accuracy when using DECT over SECT and reported that the accuracy of dose calculations based on DECT was within $\pm 4 \%$ for all tissues considered. Thus the validation of our DECT simulation supports the conclusions of our previous work and suggests that DECT based post implant dosimetry performed with clinically available scanners could yield better tissue identification and dosimetric accuracy. Extending the DECT technique to cone-beam CT (CBCT) imaging might also be of interest. Intraoperative $\mathrm{CBCT}$ based planning and dosimetry ${ }^{32,33}$ allows for online correction of poor coverage. A dual energy CBCT could thus deliver high accuracy dosimetry to the operating theatre.

\section{CONCLUSIONS}

We have evaluated the accuracy of $Z$ and $\rho_{\mathrm{e}} / \rho_{\mathrm{e}, \mathrm{w}}$ obtained from performing dual energy analysis on a pair of CT images of a RMI 465 phantom simultaneously acquired at $80 \mathrm{kVp}$ and $140 \mathrm{kVp} / \mathrm{Sn}$ by a clinical second generation dual source DECT scanner. Using a published algorithm, the accuracy on the measured $Z$, which is the additional information provided by DECT, was found to vary from $-10 \%$ for low $Z$ materials to $10 \%$ for high $Z$ materials. The accuracy on the measured $\rho_{\mathrm{e}} / \rho_{\mathrm{e}, \mathrm{w}}$ was found to be similar to what is achievable by SECT.

We were able to reproduce the measured results by simulating the DECT scanner and the measurement process with a CT simulation model. The $Z$ and $\rho_{\mathrm{e}} / \rho_{\mathrm{e}, \mathrm{w}}$ provided by our simulation agreed with the measurements within $\pm 5 \%$ for all but three inserts. We can thus consider these simulations a suitable approach to explore the use of DECT imaging in radiotherapy. 
This work is supported by Grants No. 017133 and No. 700810 of the Canadian Cancer Society. G.L. is supported by a PGSD2 scholarship from the Natural Sciences and Engineering Research Council of Canada and by the O'Brien Foundation. The authors would like to thank Jeroen Kroll and Rene Pluymakers for help with scanner operation as well as Mark Podesta for useful discussions and Siemens Healthcare for technical information.

\section{REFERENCES}

${ }^{1}$ F. Verhaegen and S. Devic, "Sensitivity study for CT image use in Monte Carlo treatment planning," Phys Med Biol 50, 937-946 (2005).

J.F. Williamson, S. Li, S. Devic, B.R. Whiting, and F.A. Lerma, "On two-parameter models of photon cross sections: application to dual-energy CT imaging," Med Phys 33, 4115-4129 (2006).

${ }^{3}$ M. Yang, G. Virshup, J. Clayton, X.R. Zhu, R. Mohan, and L. Dong, "Theoretical variance analysis of single- and dual-energy computed tomography methods for calculating proton stopping power ratios of biological tissues," Phys Med Biol 55, 1343-1362 (2010).

${ }^{4} M$. Yang, G. Virshup, J. Clayton, X.R. Zhu, R. Mohan, and L. Dong, "Does kV-MV dual-energy computed tomography have an advantage in determining proton stopping power ratios in patients?," Phys Med Biol 56, 4499-4515 (2011).

${ }^{5} \mathrm{M}$. Bazalova, J.F. Carrier, L. Beaulieu, and F. Verhaegen, "Dual-energy CT-based material extraction for tissue segmentation in Monte Carlo dose calculations," Phys Med Biol 53, 2439-2456 (2008).

${ }^{6} \mathrm{M}$. Bazalova, J.F. Carrier, L. Beaulieu, and F. Verhaegen, "Tissue segmentation in Monte Carlo treatment planning: a simulation study using dual-energy CT images," Radiother Oncol 86, 93-98 (2008).

${ }^{7} \mathrm{M}$. Saito, "Optimized low-kV spectrum of dual-energy CT equipped with high-kV tin filtration for electron density measurements," Med Phys 38, 2850-2858 (2011).

${ }^{8}$ A.N. Primak, J.C. Ramirez Giraldo, X. Liu, L. Yu, and C.H. McCollough, "Improved dual-energy material discrimination for dual-source $\mathrm{CT}$ by means of additional spectral filtration," Med Phys 36, 1359-1369 (2009).

${ }^{9}$ M.M. Goodsitt, E.G. Christodoulou, and S.C. Larson, "Accuracies of the synthesized monochromatic CT numbers and effective atomic numbers obtained with a rapid $\mathrm{kVp}$ switching dual energy CT scanner," Med Phys 38, 2222-2232 (2011).

${ }^{10} \mathrm{M}$. Torikoshi, et al., "Electron density measurement with dual-energy $\mathrm{x}$-ray CT using synchrotron radiation," Phys Med Biol 48, 673-685 (2003).

${ }^{11}$ G. Landry, P.V. Granton, B. Reniers, M.C. Ollers, L. Beaulieu, J.E. Wildberger, and F. Verhaegen, "Simulation study on potential accuracy gains from dual energy CT tissue segmentation for low-energy brachytherapy Monte Carlo dose calculations," Physics in medicine and biology 56, 6257-6278 (2011).

${ }^{12} \mathrm{G}$. Landry, et al., "Sensitivity of low energy brachytherapy Monte Carlo dose calculations to uncertainties in human tissue composition," Med Phys 37, 5188-5198 (2010).

${ }^{13}$ T.G. Flohr, et al., "Dual-source spiral CT with pitch up to 3.2 and $75 \mathrm{~ms}$ temporal resolution: image reconstruction and assessment of image quality," Med Phys 36, 5641-5653 (2009). 
${ }^{14}$ G. Poludniowski, G. Landry, F. DeBlois, P.M. Evans, and F. Verhaegen, "SpekCalc: a program to calculate photon spectra from tungsten anode x-ray tubes," Phys Med Biol 54, N433-438 (2009).

${ }^{15}$ G.G. Poludniowski, "Calculation of $x$-ray spectra emerging from an $x$-ray tube. Part II. X-ray production and filtration in x-ray targets," Med Phys 34, 2175-2186 (2007).

${ }^{16}$ G.G. Poludniowski and P.M. Evans, "Calculation of $x$-ray spectra emerging from an $x$-ray tube. Part I. electron penetration characteristics in x-ray targets," Med Phys 34, 2164-2174 (2007).

${ }^{17}$ R.L. Kruger, C.H. McCollough, and F.E. Zink, "Measurement of half-value layer in x-ray CT: a comparison of two noninvasive techniques," Med Phys 27, 1915-1919 (2000).

${ }^{18}$ B. Heismann and M. Balda, "Quantitative image-based spectral reconstruction for computed tomography," Med Phys 36, 4471-4485 (2009).

${ }^{19}$ G. Landry, F. DeBlois, M. Bazalova, and F. Verhaegen, MO-D-303A-06: ImaSim, An Animated Tool for Teaching Imaging. Vol. 36. 2009: AAPM. 2696-2697.

${ }^{20}$ M.J. Berger, J.H. Hubbell, S.M. Seltzer, J. Chang, and J.S. Coursey, XCOM: Photon Cross Sections Database. 2005, NIST.

${ }^{21} Y$. Watanabe, "Derivation of linear attenuation coefficients from CT numbers for low-energy photons," Phys Med Biol 44, 2201-2211 (1999).

${ }^{22}$ L.A. Shepp and B.F. Logan, "Reconstructing Interior Head Tissue from X-Ray Transmissions," Nuclear Science, IEEE Transactions on 21, 228-236 (1974).

${ }^{23}$ R.G. Stock, N.N. Stone, A. Tabert, C. lannuzzi, and J.K. DeWyngaert, "A dose-response study for I-125 prostate implants," Int J Radiat Oncol Biol Phys 41, 101-108 (1998).

${ }^{24} \mathrm{D}$. Vordermark, et al., "Prospective evaluation of quality of life after permanent prostate brachytherapy with 1-125: importance of baseline symptoms and of prostate-V150," Radiother Oncol 91, 217-224 (2009).

${ }^{25} \mathrm{~F}$. Guedea, et al., "Patterns of care for brachytherapy in Europe: updated results," Radiother Oncol 97, 514-520 (2010).

${ }^{26}$ M.J. Rivard, et al., "Update of AAPM Task Group No. 43 Report: A revised AAPM protocol for brachytherapy dose calculations," Med Phys 31, 633-674 (2004).

${ }^{27}$ R. Nath, L.L. Anderson, G. Luxton, K.A. Weaver, J.F. Williamson, and A.S. Meigooni, "Dosimetry of interstitial brachytherapy sources: recommendations of the AAPM Radiation Therapy Committee Task Group No. 43. American Association of Physicists in Medicine," Med Phys 22, 209-234 (1995).

${ }^{28}$ G.S. Burns and D.E. Raeside, "The accuracy of single-seed dose superposition for I-125 implants," Med Phys 16, 627-631 (1989).

${ }^{29}$ J.F. Carrier, M. D'Amours, F. Verhaegen, B. Reniers, A.G. Martin, E. Vigneault, and L. Beaulieu, "Postimplant dosimetry using a Monte Carlo dose calculation engine: a new clinical standard," Int J Radiat Oncol Biol Phys 68, 1190-1198 (2007).

${ }^{30}$ H. Afsharpour, J.-P. Pignol, B. Keller, J.-F. Carrier, B. Reniers, F. Verhaegen, and L. Beaulieu, "Influence of breast composition and interseed attenuation in dose calculations for postimplant assessment of permanent breast ${ }^{103}$ Pd seed implant," Phys Med Biol 55, 4547-4561 (2010).

${ }^{31}$ G. Landry, P.V. Granton, B. Reniers, O. M.C., L. Beaulieu, J.E. Wildberger, and F. Verhaegen, "Simulation study on potential accuracy gains from dual energy CT tissue segmentation for low energy brachytherapy Monte Carlo dose calculations article in press," Phys Med Biol (2011).

${ }^{32}$ H. Westendorp, C.J. Hoekstra, A. van't Riet, A.W. Minken, and J.J. Immerzeel, "Intraoperative adaptive brachytherapy of iodine- 125 prostate implants guided by C-arm cone-beam computed tomography-based dosimetry," Brachytherapy 6, 231-237 (2007). 
${ }^{33}$ A. Polo, C. Salembier, J. Venselaar, and P. Hoskin, "Review of intraoperative imaging and planning techniques in permanent seed prostate brachytherapy," Radiother Oncol 94, 1223 (2010). 



\section{CHAPTER}

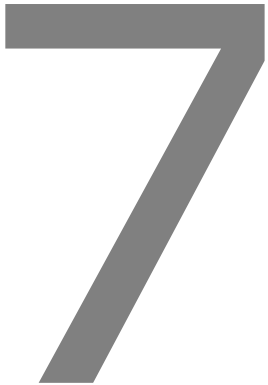

Systematic evaluation of noise levels from single and dual energy acquisitions with a dual source CT scanner: comparing iterative and analytical reconstruction methods

Guillaume Landry, Mathieu Gaudreault, Wouter van Elmpt, Joachim E. Wildberger, Frank Verhaegen

Under review 


\section{ABSTRACT}

Objectives The goal of this study was to evaluate the noise reduction achievable from dual energy CT imaging (DECT) using a dual source CT scanner and the vendor's filtered backprojection (FBP) and iterative image reconstruction algorithms. We aimed at evaluating the data in the context of imaging for radiotherapy treatment planning and dose calculation, where accurate and precise quantification of electron density $\rho_{\mathrm{e}}$ and effective atomic number $Z_{\text {eff }}$ is required.

Materials and methods A second generation dual source CT scanner was used to scan a phantom containing tissue mimicking inserts. DECT scans were acquired at $80 \mathrm{kVp}$ and 140Sn kVp (where Sn stands for tin filtration which offers better spectral separation) and $100 \mathrm{kVp}$ and $140 \mathrm{Sn} \mathrm{kVp}$, using same values of the computed tomography dose index $\mathrm{CTDI}_{\mathrm{vol}}$ for both settings. Four $\mathrm{CTDI}_{\mathrm{vol}}$ levels were investigated. Images were reconstructed using FBP and sinogram affirmed iterative reconstruction (SAFIRE) with strength 1,3 and 5 . From DECT scans two quantities were derived, $Z_{\text {eff }}$ and $\rho_{\mathrm{e}}$. Mean values and standard deviations were obtained for each quantity in each insert using regions of interest (ROI). Reconstruction times were compared for FBP and SAFIRE. $D E C T$ images were used to assign tissue types for each voxel based on reference values for $Z_{\text {eff }}$ and $\rho_{\mathrm{e}}$ derived from manufacturer data. The amount of improperly assigned voxels was quantified.

Results SAFIRE reconstruction times were found to be longer by roughly a factor 3 for all strengths. Standard deviations for $Z_{\text {eff }}$ and $\rho_{\mathrm{e}}$ were reduced when using SAFIRE, up to a factor $\sim 1.8$ with strength 5 . Standard deviations on $Z_{\text {eff }}$ and $\rho_{\mathrm{e}}$ as low as 0.15 and 0.006 were achieved. This led to highly accurate material type assignment.

Conclusions This phantom study showed that the SAFIRE image reconstruction algorithm provided reduced standard deviations while preserving mean values and improved material type assignment. 


\section{INTRODUCTION}

In diagnostic imaging, dual energy computed tomography (DECT) can provide more information than traditional single energy CT (SECT) by imaging at a high and low kVp. This additional information can be used for tissue identification ${ }^{1}$, contrast agent quantification $^{2}$, differentiation of contrast agent and bony tissues ${ }^{3,4}$ or the reconstruction of optimal mono-energetic images for metal artefact reduction ${ }^{5,6}$.

Applications of DECT outside diagnostic radiology have also been explored. The ability of DECT to yield estimates of a tissue's electron density $\rho_{\mathrm{e}}$ and effective atomic number $Z_{\text {eff }}$ has benefits for specific radiotherapy applications ${ }^{7-9}$. In those applications the identification of tissue properties (i.e. $\rho_{\mathrm{e}}$ and $Z_{\text {eff }}$ ) can be important for accurate radiotherapy treatment dose calculation. Currently, one of the main limitations of DECT scanning for radiotherapy where tissue composition is important is the high noise levels observed on the $Z_{\text {eff }}$ images ${ }^{8}$.

Sinogram affirmed iterative reconstruction (SAFIRE, Siemens Healthcare, Forchheim, Germany) has recently been introduced as an alternative to standard filtered backprojection reconstruction (FBP). SAFIRE has been shown to yield images of quality equivalent to those obtained from FBP but at a reduced radiation dose level ${ }^{10-12}$ and with improved low contrast detectability ${ }^{13}$. For radiology applications this leads to important dose savings for the patient population undergoing CT scanning. For radiotherapy purposes, where radiation dose should be evaluated in the context of the high treatment dose, the imaging dose can be higher than in a solely diagnostic setting. In radiotherapy, the noise reduction capabilities of SAFIRE at any dose level can be exploited to improve accuracy of radiotherapy treatment planning.

This work aimed at investigating the gain of improved $\rho_{\mathrm{e}}$ and $Z_{\text {eff }}$ estimation from SAFIRE over FBP by performing a phantom study. We compared quantitatively the performance of SAFIRE and FBP for DECT scanning at several dose levels by estimating the noise reduction from iterative reconstruction and its impact on material type assignment. The aim of this work is to provide a comprehensive source of information on DECT noise levels for FBP and SAFIRE reconstruction.

\section{MATERIALS AND METHODS}

\section{Phantom scans and reconstruction}

A phantom with an elliptical cross section $30 \mathrm{~cm}$ long, $30 \mathrm{~cm}$ wide and $20 \mathrm{~cm}$ thick (anterior-posterior) was employed in this study (Model 002H5, CIRS Inc., Norfolk, VA). The phantom consisted of tissue equivalent epoxy material and accommodated up to five cylindrical inserts with a diameter of $2.5 \mathrm{~cm}$. The inserts consisted of epoxy materials mimicking various tissues with respect to electron density: bone, lung (inhale), adipose, muscle and plastic water (background) (product 002ED, CIRS Inc., Norfolk, VA). A DECT image of the phantom is shown in Figure 7.1. The elemental composition 
and electron density of each insert as well as the bulk phantom material were provided by the manufacturer.

The phantom was scanned using a second generation dual source CT scanner (SOMATOM Definition FLASH, Siemens Healthcare). Two series of DECT scans were performed at $80 \mathrm{kVp} / 140 \mathrm{Sn} \mathrm{kVp}$ and $100 \mathrm{kVp} / 140 \mathrm{Sn} \mathrm{kVp}$; the $140 \mathrm{Sn} \mathrm{kVp}$ beam was filtered by a Sn filter which offers better spectral separation by preferentially attenuating low energy photons ${ }^{14}$. The exposure levels from the institutional DECT protocol for abdomen using $80 \mathrm{kVp}$ (238 mAs eff.) and 140Sn kVp (92 mAs eff.) with combined computed tomography dose index $\mathrm{CTDI}_{\mathrm{vol}}=9.2 \mathrm{mGy}$ (body phantom) were chosen as standard. Each $80 \mathrm{kVp} / 140 \mathrm{SnkVp}$ DECT scan series consisted of 4 different exposure levels $(0.5 \times, 1 \times, 2 \times$ and $4 \times$ the standard exposure described above). The $4 \times$ exposure was the maximum allowed by the scanner control software. For the $100 \mathrm{kVp} / 140 \mathrm{Sn} \mathrm{kVp}$ the standard exposures were chosen to yield the same $C_{T D I}{ }_{\mathrm{vol}}=9.2 \mathrm{mGy}$ and were 114 mAs eff. at $100 \mathrm{kVp}$ and $88 \mathrm{mAs}$ eff. at $140 \mathrm{kVp} / \mathrm{Sn}$. The $100 \mathrm{kVp} / 140 \mathrm{Sn} \mathrm{kVp}$ scan series consisted of three exposure levels $(0.5 \times, 1 \times$ and $2 \times$ the exposures corresponding to $\mathrm{CTDI}_{\mathrm{vol}}=9.2 \mathrm{mGy}$ ). . The collimation was set to $32 \times 0.6 \mathrm{~mm}, \mathrm{mAs}$ modulation was disabled and pitch was 0.2 . The low pitch value was necessary to perform scanning at the high exposure levels due to the limitations of the $x$-ray output. As this pitch is below the recommended minimum value (the low pitch value combined with the lowest dose can lead to a low $x$-ray fluence reaching the detector and may lead to non-linearity) we additionally scanned the phantom with a pitch of $0.4,0.6$ and 0.8 at the lowest exposures $(0.5 \times)$.

The data sets were reconstructed with $1 \mathrm{~mm}$ and $3 \mathrm{~mm}$ slice thicknesses at $1 \mathrm{~mm}$ and $3 \mathrm{~mm}$ intervals respectively with no overlap. For each exposure level of each series, images were reconstructed with a standard kernel (FBP: D30f for DECT) as well as with iterative reconstruction (SAFIRE: Q30f for DECT) using strengths of 1,3 and 5 . The SAFIRE strength governs the level of noise in the images, with higher strength resulting in lower noise. The $512 \times 512$ images covered a field of view of $35 \mathrm{~cm}$ diameter.

The Q30f kernel is designed in a way that the total modulation transfer function of a high contrast object, e.g. a thin wire, considering both the effect of the convolution kernel and of the regularization term in the iterative reconstruction, is similar to the modulation function of D30f. The $50 \%$ and $10 \%$ values of the modulation transfer function of the Q30f kernel are the same as for the D30f kernel. It is thus fair to directly compare the noise in the images obtained from FBP with D30f and SAFIRE using Q30f (private communication with Siemens Medical).

The time to reconstruct $1001 \mathrm{~mm}$ slices covering a $10 \mathrm{~cm}$ long scan volume using D30f and Q30f with strength 1, 3 and 5 was also measured at the standard dose for DECT acquisitions.

\section{Data processing}

We employed an in-house, radiotherapy oriented, image based $\rho_{\mathrm{e}}-Z_{\text {eff }}$ algorithm ${ }^{7}$ providing the electron density relative to water $\rho_{\mathrm{e}}$ and effective atomic number $Z_{\text {eff }}$ which was previously validated ${ }^{8}$. The algorithm relies on a parameterization of the 
linear attenuation coefficient $\mu(E)$ as a function of $\rho_{\mathrm{e}}, Z_{\text {eff }}$ and photon energy $E$. The algorithm solves for $\rho_{\mathrm{e}}$ and $Z_{\text {eff }}$ using the measured high (140Sn) kVp and low (80 or $100) \mathrm{kVp}$ images and prior knowledge of the photon spectra.

Regions of interest (ROI) in the central axial slice covering $90 \%$ of the image area of each tissue mimicking insert were used to assess the mean and standard deviation of the quantities of interest (CT numbers, $\rho_{\mathrm{e}}, Z_{\text {eff }}$ ) for all experiments. The ROls were not affected by edge effects. For the combination of one exposure level, slice thickness, image reconstruction algorithm and insert we thus have mean and standard deviation for $\mathrm{HU}_{140}, \mathrm{HU}_{100}, \mathrm{HU}_{80}, Z_{\text {eff }}$ and $\rho_{\mathrm{e}}$.

To evaluate the impact of noise on the assignment of material composition a procedure to link each voxel with a material composition was performed for each $\mathrm{kVp}$ pair, exposure level, slice thickness and reconstruction setting. A lookup table approach was employed where for each voxel, for which we have $\left[Z_{\text {eff, }}, \rho_{\text {e }}\right]_{\text {measured, }}$ the $2 \mathrm{D}$ Mahalanobis distance to the $\left[Z_{\text {eff, }}, \rho_{\mathrm{e}}\right]_{\text {reference }}$ of each reference material of Table 1 was computed. The covariance matrix required to compute the Mahalanobis distance was estimated from a distribution of voxels selected in the bulk material of the phantom. The reference material minimizing the Mahalanobis distance was assigned to voxels and the amount of misassignment was quantified.

\section{RESULTS AND DISCUSSION}

Figure 7.1(A,B) present DECT $\rho_{\mathrm{e}}$ images of the phantom reconstructed with D30f (FBP) and Q30f $\backslash 5$ (SAFIRE) and shows reduced noise amplitude in the Q30f $\backslash 5$ image. The location of the inserts and the ROls used in the data analysis are shown on Figure 7.1(A).

Image reconstruction timing was 0.34 s per slice for the DECT FBP protocol. Reconstruction time increased to $1.02 \mathrm{~s}$ per slice using the SAFIRE approach, with no differences at the different strength levels.

Figure 7.2 presents the results of the $80 \mathrm{kVp} / 140 \mathrm{SnkVp}$ DECT scan series for the muscle insert of the phantom. For $\mathrm{HU}_{140}, \mathrm{HU}_{80}, Z_{\text {eff, }}$ and $\rho_{\mathrm{e}}$, iterative reconstruction provided similar mean HU values (within standard deviation) as FBP. Within the standard deviations nearly all values agree with the expected values (full horizontal line). The Q30f $\backslash 1$ reconstruction provided similar standard deviations as D30f, while Q30f $\backslash 3$ and $Q 30 f \backslash 5$ reconstructions lowered the standard deviations in all cases. 

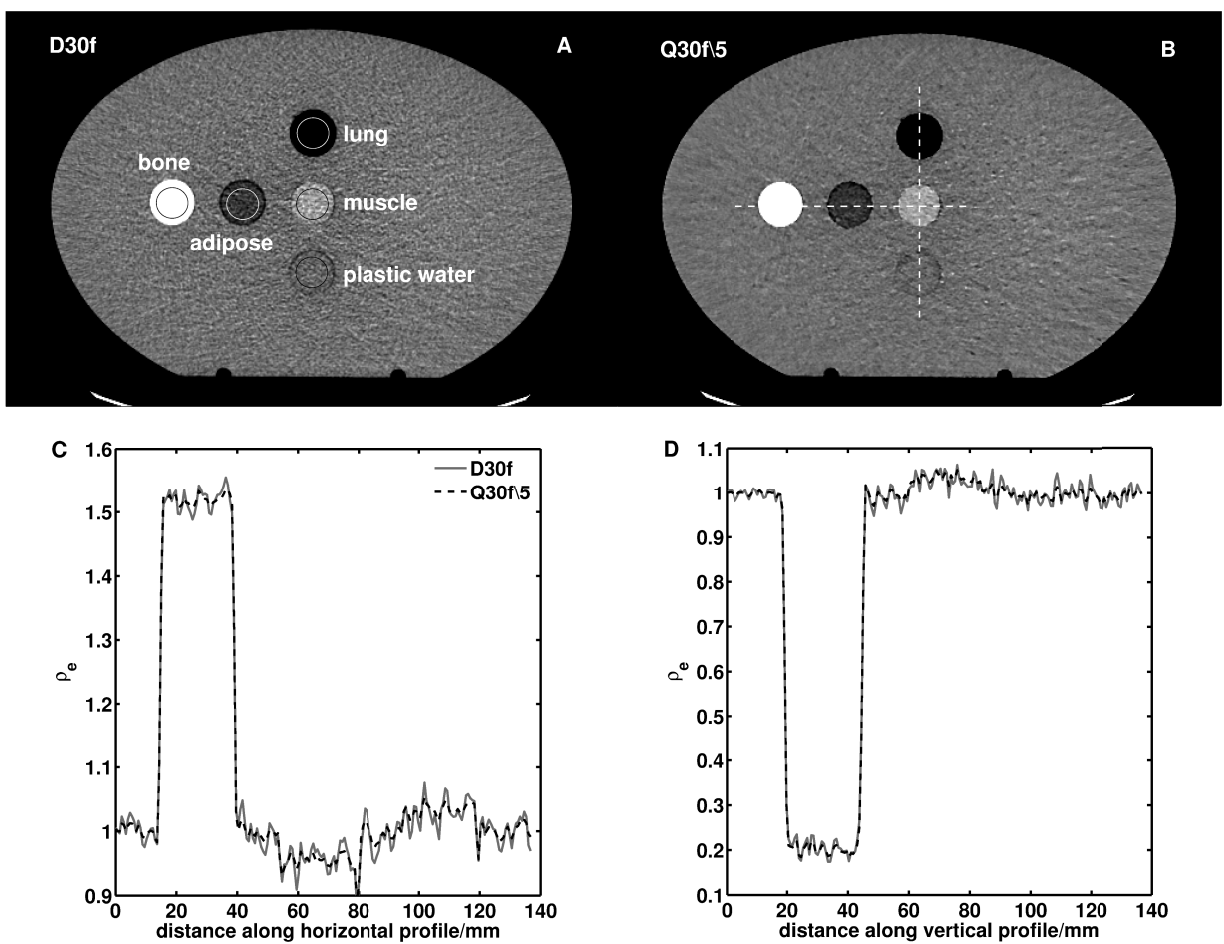

Figure 7.1 Central axial DECT $\rho_{e}$ image of the phantom reconstructed with (A) D30f and (B) Q30f $\backslash 5$ with standard dose and $3 \mathrm{~mm}$ slice thickness. Window and level set to 0.2 and 1 units of $\rho_{e}$. The circles indicate the ROls used to derive mean and standard deviation. (C) horizontal and (D) vertical profiles along the lines shown in (B) for the D30f and Q30f $\backslash 5$ images.

Figure 7.3 shows the standard deviations of $Z_{\text {eff }}$ and $\rho_{\mathrm{e}}$ as a function of reconstruction method and exposure for $1 \mathrm{~mm}$ slice thickness. The use of higher SAFIRE strength yielded lower standard deviations. For all reconstruction methods, increasing exposure or increasing slice thickness reduced the standard deviation. For $\mathrm{HU}_{140}, \mathrm{HU}_{80}, Z_{\text {eff }}$ and $\rho_{\mathrm{e}}$ it was found that the noise levels for the muscle insert obtained using a standard dose and the Q30f $\backslash 5$ reconstruction were equivalent to those obtained with the maximum exposure and the D30f reconstruction.

The lowest standard deviations for $\mathrm{HU}_{140}, \mathrm{HU}_{80}, Z_{\text {eff }}$ and $\rho_{\mathrm{e}}$ were $4 \mathrm{HU}, 5 \mathrm{HU}, 0.15$ and 0.006 respectively, obtained using a $3 \mathrm{~mm}$ slice thickness, the maximum exposure and the Q30f $\backslash 5$ reconstruction. Table 7.1 shows the results of $Z_{\text {eff }}$ and $\rho_{\mathrm{e}}$ for these conditions and compared to reference values calculated from the manufacturer provided composition. 

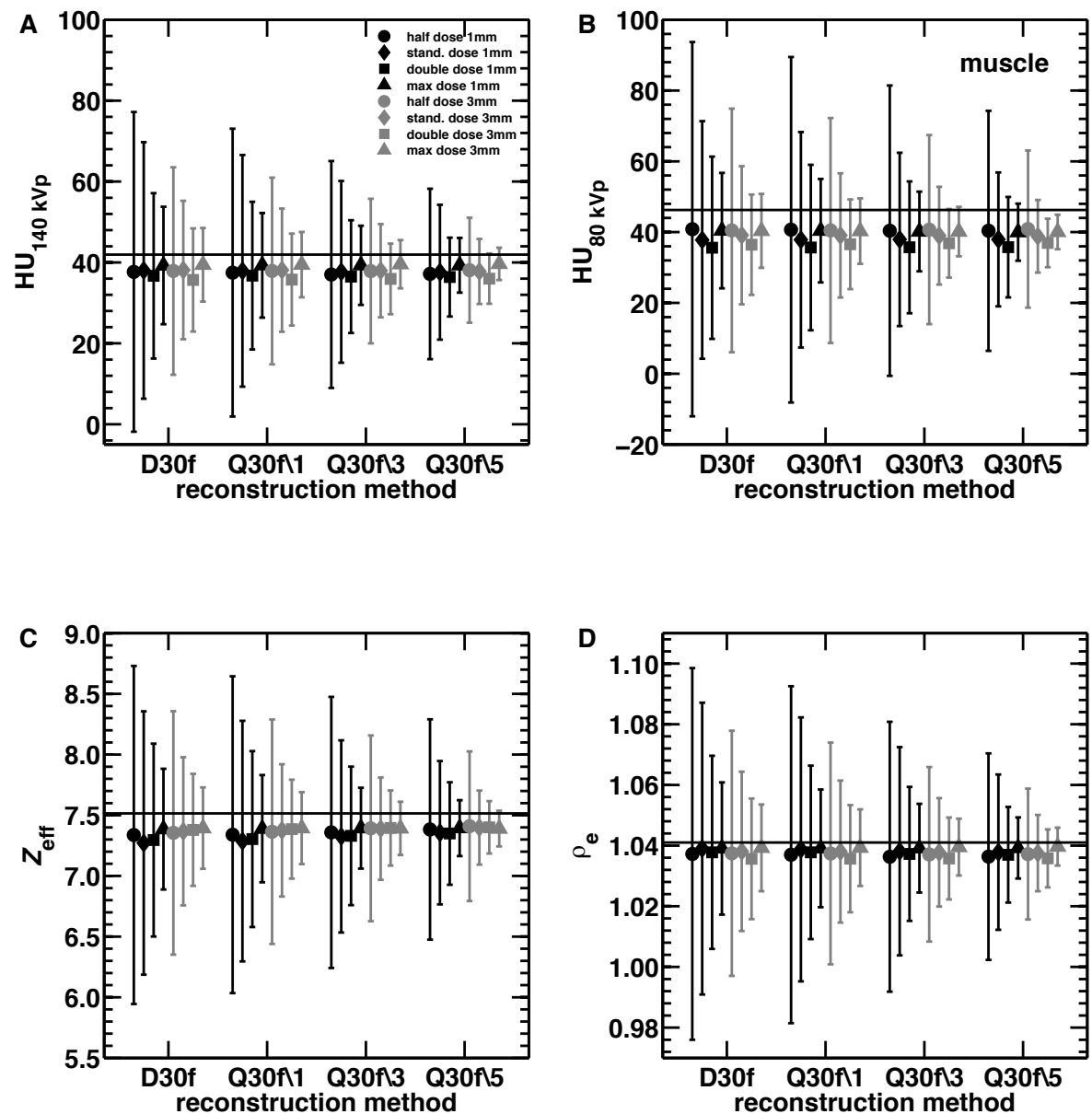

Figure 7.2: Mean and standard deviation (error bars) of CT numbers from the (A) $140 \mathrm{Sn} k \mathrm{~V} p$ and (B) $80 \mathrm{kVp}$ images, $(C) Z_{\text {eff }}$ and (D) $\rho_{e}$ of a ROI centered on the muscle insert of the phantom from the $80 \mathrm{kVp} / 140 \mathrm{SnkVp}$ series of DECT scans at four exposure levels (half, standard, double and maximum exposures) and various filters D30f and $Q 30 f \backslash N$ with $N=1,3,5$. Black/gray symbols indicate $1 \mathrm{~mm} / 3 \mathrm{~mm}$ slice thickness. The analysis was performed on a single, central axial $512 \times 512$ slice. Horizontal lines represent the reference values calculated using our calculation of the system's photon spectra and detector response and the manufacturerprovided insert composition data. 

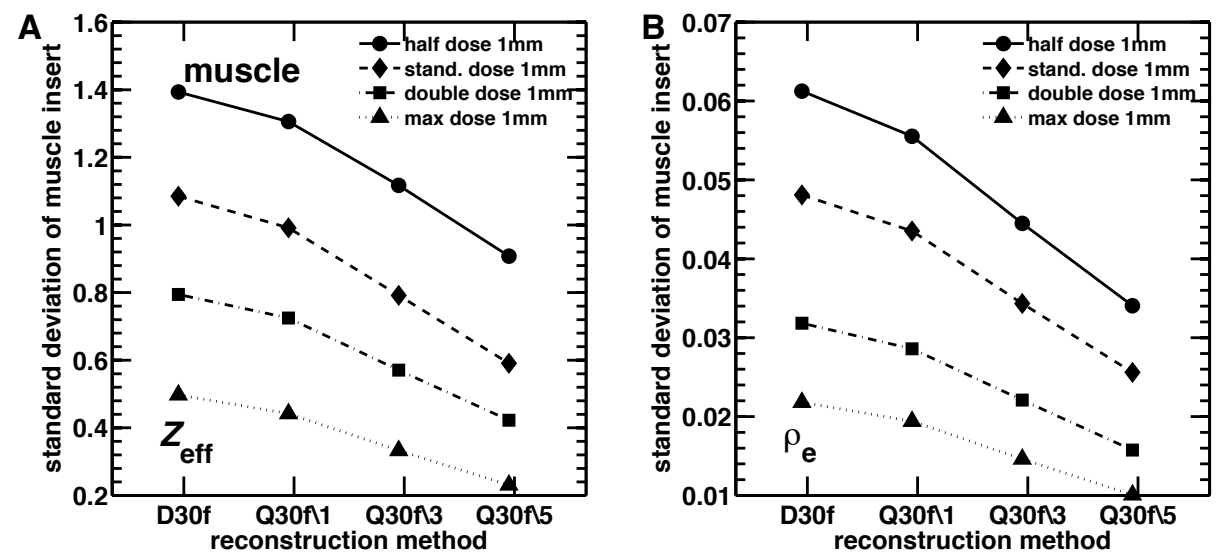

Figure 7.3: Standard deviations of the muscle insert of (A) $Z_{\text {eff }}$ and (B) $\rho_{e}$ images from the $80 \mathrm{kVp} / 140 \mathrm{SnkVp}$ DECT scan series. Each curve belongs to an exposure level and for each exposure level the standard deviation is presented as a function of reconstruction method. $1 \mathrm{~mm}$ slice thickness data is presented.

Table 7.1: Reference values for $Z_{\text {eff }}$ and $\rho_{e}$ for the phantom inserts compared to those obtained from the lowest standard deviation scans (columns 2 and 4).

\begin{tabular}{lllll}
\hline \hline insert & $Z_{\text {eff }}$ reference & $Z_{\text {eff }}$ measured & $\rho_{\mathrm{e}}$ reference & $\rho_{\mathrm{e}}$ measured \\
\hline lung & 7.24 & $7.25 \pm 0.67$ & 0.204 & $0.195 \pm 0.006$ \\
adipose & 6.44 & $6.49 \pm 0.18$ & 0.956 & $0.954 \pm 0.006$ \\
plastic water & 7.55 & $7.44 \pm 0.12$ & 1.000 & $0.992 \pm 0.005$ \\
muscle & 7.59 & $7.43 \pm 0.15$ & 1.041 & $1.039 \pm 0.006$ \\
bone & 11.90 & $11.05 \pm 0.05$ & 1.507 & $1.517 \pm 0.006$ \\
\hline \hline
\end{tabular}

When using a pitch of 0.2 , the half exposure DECT scans showed mean CT numbers which deviated from those obtained with higher exposures for all reconstruction methods. Employing a higher pitch corrected this problem, which was most severe for the bone insert at $80 \mathrm{kVp}$. For other exposures the results were independent of the pitch value.

Table 7.2 presents the results, for the muscle insert, of the $100 \mathrm{kVp} / 140 \mathrm{SnkVp}$ DECT scan series. In general the standard deviations of the DECT derived quantities $Z_{\text {eff, }}$ $\rho_{\mathrm{e}}$ at $100 \mathrm{kVp} / 140 \mathrm{SnkVp}$ were found to be higher than those obtained from the $80 \mathrm{kVp} / 140 \mathrm{SnkVp}$ DECT scan series. 
Table 7.2: Mean and standard deviation of $\mathrm{HU}_{140}, \mathrm{HU}_{100,} \mathrm{Z}_{\text {eff }}$ and $\rho_{e}$ of a ROI centered on the muscle insert of the phantom from the $100 \mathrm{kVp} / 140 \mathrm{SnkVp}$ series of DECT scans at three exposure levels: half, standard and double exposures. Data for $1 \mathrm{~mm} / 3 \mathrm{~mm}$ slice thickness are presented. The analysis was performed on a single, central axial $512 \times 512$ slice.

\begin{tabular}{|c|c|c|c|c|c|c|c|c|c|}
\hline & & HU140 & & HU100 & & $Z_{\text {eff }}$ & & $\rho_{\mathrm{e}}$ & \\
\hline $\begin{array}{l}\text { recon. } \\
\text { method }\end{array}$ & dose & $1 \mathrm{~mm}$ & $3 \mathrm{~mm}$ & $1 \mathrm{~mm}$ & $3 \mathrm{~mm}$ & $1 \mathrm{~mm}$ & $3 \mathrm{~mm}$ & $1 \mathrm{~mm}$ & $3 \mathrm{~mm}$ \\
\hline \multirow[t]{3}{*}{ D30f } & half & $38 \pm 43$ & $37 \pm 29$ & $39 \pm 43$ & $39 \pm 27$ & $7.31 \pm 1.68$ & $7.33 \pm 1.19$ & $1.039 \pm 0.075$ & $1.036 \pm 0.049$ \\
\hline & standard & $35 \pm 30$ & $35 \pm 19$ & $36 \pm 31$ & $37 \pm 21$ & $7.30 \pm 1.30$ & $7.36 \pm 0.95$ & $1.035 \pm 0.052$ & $1.034 \pm 0.034$ \\
\hline & double & $35 \pm 21$ & $35 \pm 13$ & $39 \pm 19$ & $41 \pm 11$ & $7.41 \pm 0.99$ & $7.50 \pm 0.57$ & $1.033 \pm 0.038$ & $1.032 \pm 0.023$ \\
\hline \multirow[t]{3}{*}{ Q30f/1 } & half & $38 \pm 39$ & $37 \pm 27$ & $39 \pm 39$ & $39 \pm 25$ & $7.29 \pm 1.58$ & $7.34 \pm 1.10$ & $1.039 \pm 0.068$ & $1.036 \pm 0.045$ \\
\hline & standard & $34 \pm 27$ & $35 \pm 17$ & $36 \pm 28$ & $37 \pm 19$ & $7.30 \pm 1.21$ & $7.37 \pm 0.86$ & $1.034 \pm 0.047$ & $1.034 \pm 0.031$ \\
\hline & double & $35 \pm 19$ & $35 \pm 11$ & $39 \pm 17$ & $41 \pm 10$ & $7.42 \pm 0.89$ & $7.51 \pm 0.50$ & $1.033 \pm 0.034$ & $1.032 \pm 0.020$ \\
\hline \multirow[t]{3}{*}{ Q30f/3 } & half & $38 \pm 32$ & $36 \pm 21$ & $39 \pm 32$ & $38 \pm 20$ & $7.29 \pm 1.37$ & $7.37 \pm 0.91$ & $1.039 \pm 0.055$ & $1.035 \pm 0.036$ \\
\hline & standard & $34 \pm 22$ & $35 \pm 13$ & $36 \pm 23$ & $37 \pm 15$ & $7.33 \pm 1.01$ & $7.39 \pm 0.68$ & $1.034 \pm 0.037$ & $1.034 \pm 0.024$ \\
\hline & double & $35 \pm 15$ & $35 \pm 8$ & $39 \pm 13$ & $40 \pm 7$ & $7.46 \pm 0.67$ & $7.52 \pm 0.37$ & $1.033 \pm 0.026$ & $1.033 \pm 0.015$ \\
\hline \multirow[t]{3}{*}{ Q30f/5 } & half & $38 \pm 24$ & $36 \pm 17$ & $39 \pm 25$ & $38 \pm 16$ & $7.30 \pm 1.12$ & $7.40 \pm 0.70$ & $1.038 \pm 0.043$ & $1.035 \pm 0.028$ \\
\hline & standard & $34 \pm 16$ & $35 \pm 10$ & $35 \pm 19$ & $37 \pm 12$ & $7.36 \pm 0.80$ & $7.39 \pm 0.51$ & $1.033 \pm 0.028$ & $1.035 \pm 0.018$ \\
\hline & double & $35 \pm 10$ & $36 \pm 6$ & $39 \pm 9$ & $41 \pm 5$ & $7.49 \pm 0.46$ & $7.52 \pm 0.23$ & $1.033 \pm 0.018$ & $1.033 \pm 0.010$ \\
\hline
\end{tabular}

Figure 7.4(A) presents the results of the material assignment exercise for the $80 \mathrm{kVp} / 140 \mathrm{SnkVp}$ DECT scan series. In general decreasing imaging noise by increasing the slice thickness or exposure reduces the number of voxels for which the material assignment is erroneous. When using the SAFIRE reconstruction method with strength 5 all voxels were correctly assigned with exposures yielding a CTDI ${ }_{\text {vol }}$ of about $40 \mathrm{mGy}$ and $3 \mathrm{~mm}$ slice thickness.

All voxels in the lung and bone inserts were correctly assigned for all the reconstruction methods, slice thicknesses, doses and pitches considered. This was expected as they are well isolated in the $\left[Z_{\text {eff }}, \rho_{\mathrm{e}}\right]$ space (see Table 7.1). Material assignation was not always exact for voxels in the muscle and water inserts, as they lie closer together in the $\left[Z_{\text {eff, }} \rho_{\mathrm{e}}\right]$ space. From Table 7.1 we see they have the same $Z_{\text {eff }}$ and a $4 \% \rho_{\mathrm{e}}$ difference. In particular, around $60 \%$ of the voxels were properly attributed in the worst case scenario (D30f, dose $=4.6 \mathrm{mGy}$, and slice thickness of $1 \mathrm{~mm}$ for the $100 \mathrm{kVp} / 140 \mathrm{SnkVp}$ DECT scan series) for both inserts. Material assignation was better for the adipose insert for which around $75 \%$ of the voxels were properly assigned for the same parameters.

Results of the percentage of voxels not properly assigned are shown in Figure 7.4(B) as a function of the standard deviation of $Z_{\text {eff }}$ averaged over all inserts for all the reconstruction methods considered. Increasing the dose or the slice thickness both decreases the standard deviation of $Z_{\text {eff }}$ and the percentage of voxels improperly assigned. In particular, the standard deviation of $Z_{\text {eff }}$ and the percentage of voxels not correctly assigned are respectively smaller than $2 \%$ and $5 \%$ for a slice thickness of $3 \mathrm{~mm}$ with the SAFIRE reconstruction method with strength 5 at all exposures.

Mahnken et al. ${ }^{15}$ state that a maximum noise level of 0.2 units in $Z_{\text {eff }}$ is required for differentiation of soft tissues, which is in agreement with our findings if we consider plastic water and muscle tissue substitute to be surrogates of closely related soft tissues. 

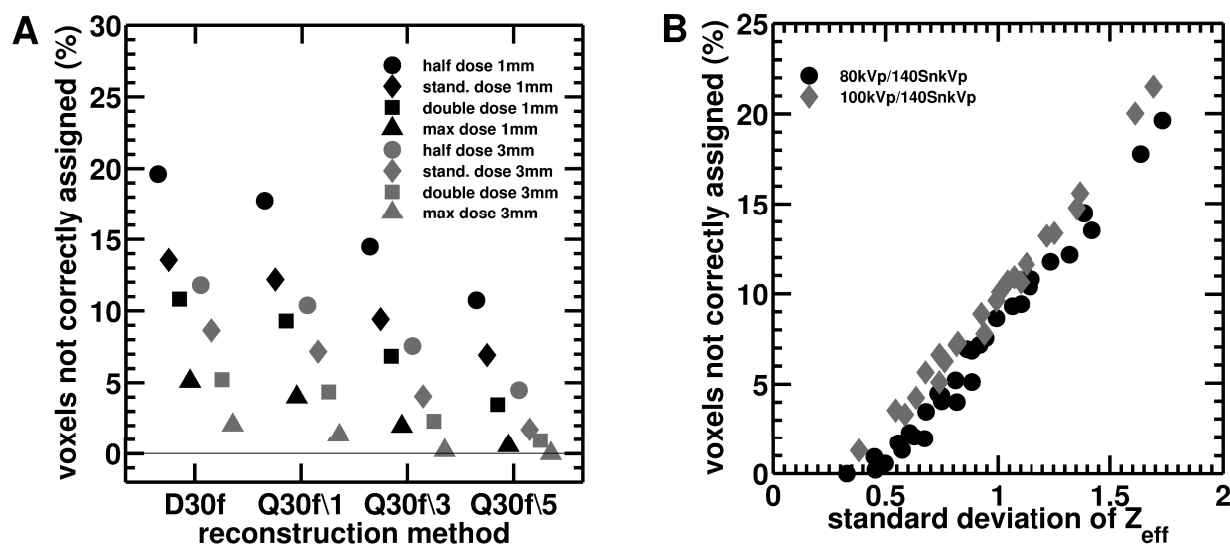

Figure 7.4: (A) Percentage of voxels in all ROIs from the 80kVp/140SnkVp DECT scan series which have been improperly assigned a material type. (B) Percentage of voxels in all ROls from the $80 \mathrm{kVp} / 140 \mathrm{SnkVp}$ and $100 \mathrm{kVp} / 140 \mathrm{SnkVp}$ DECT scan series which have not been correctly assigned a material type as a function of the standard deviation of $Z_{\text {eff }}$ averaged over all the inserts for all the reconstruction methods and scan settings considered.

\section{CONCLUSION}

Our results demonstrate that reconstructing images using SAFIRE yields similar mean CT numbers, $Z_{\text {eff }}$ and $\rho_{\mathrm{e}}$ when compared to FBP. This is important for DECT applications where accurate quantification is desired.

From a radiotherapy perspective, where minimal noise on $Z_{\text {eff }}$ and $\rho_{\mathrm{e}}$ is desired, SAFIRE can be combined with an imaging dose increase to reduce noise levels on these quantities to 0.15 and 0.006 respectively (maximum exposure, $3 \mathrm{~mm}$ slice thickness, SAFIRE strength 5, muscle insert) which yields highly accurate material assignment for this phantom.

This work provides a comprehensive and quantitative source of data on the current noise levels obtainable with SAFIRE reconstruction for DECT scanning.

\section{ACKNOWLEDGEMENTS}

We thank Dr. Bernhard Schmidt and Dr. Thomas Flohr from Siemens Medical for providing information on the operation of the DECT scanner. We acknowledge Vladimir Varchena of CIRS for divulging phantom compositions. G.L. would like to acknowledge the O'Brien Foundation for funding. 


\section{REFERENCES}

${ }^{1}$ T.R. Johnson, et al., "Material differentiation by dual energy CT: initial experience," Eur Radiol 17, 1510-1517 (2007).

${ }^{2}$ S. Feuerlein, T.J. Heye, M.R. Bashir, and D.T. Boll, "lodine quantification using dual-energy multidetector computed tomography imaging: phantom study assessing the impact of iterative reconstruction schemes and patient habitus on accuracy," Invest Radiol 47, 656661 (2012).

${ }^{3}$ W.H. Sommer, T.R. Johnson, C.R. Becker, E. Arnoldi, H. Kramer, M.F. Reiser, and K. Nikolaou, "The value of dual-energy bone removal in maximum intensity projections of lower extremity computed tomography angiography," Invest Radiol 44, 285-292 (2009).

${ }^{4}$ D. Morhard, C. Fink, A. Graser, M.F. Reiser, C. Becker, and T.R. Johnson, "Cervical and cranial computed tomographic angiography with automated bone removal: dual energy computed tomography versus standard computed tomography," Invest Radiol 44, 293-297 (2009).

${ }^{5} \mathrm{C}$. Zhou, et al., "Monoenergetic imaging of dual-energy CT reduces artifacts from implanted metal orthopedic devices in patients with factures," Acad Radiol 18, 1252-1257 (2011).

${ }^{6}$ F.G. Meinel, B. Bischoff, Q. Zhang, F. Bamberg, M.F. Reiser, and T.R. Johnson, "Metal artifact reduction by dual-energy computed tomography using energetic extrapolation: a systematically optimized protocol," Invest Radiol 47, 406-414 (2012).

${ }^{7}$ M. Bazalova, J.F. Carrier, L. Beaulieu, and F. Verhaegen, "Dual-energy CT-based material extraction for tissue segmentation in Monte Carlo dose calculations," Phys Med Biol 53, 2439-2456 (2008).

${ }^{8}$ G. Landry, B. Reniers, P.V. Granton, B. van Rooijen, L. Beaulieu, J.E. Wildberger, and F. Verhaegen, "Extracting atomic numbers and electron densities from a dual source dual energy CT scanner: Experiments and a simulation model," Radiotherapy and Oncology 100, 375-379 (2011).

${ }^{9}$ M. Yang, G. Virshup, J. Clayton, X.R. Zhu, R. Mohan, and L. Dong, "Theoretical variance analysis of single- and dual-energy computed tomography methods for calculating proton stopping power ratios of biological tissues," Phys Med Biol 55, 1343-1362 (2010).

${ }^{10} \mathrm{~A}$. Winklehner, et al., "Raw data-based iterative reconstruction in body CTA: evaluation of radiation dose saving potential," Eur Radiol 21, 2521-2526 (2011).

${ }^{11} \mathrm{~A}$. Moscariello, et al., "Coronary CT angiography: image quality, diagnostic accuracy, and potential for radiation dose reduction using a novel iterative image reconstruction technique-comparison with traditional filtered back projection," Eur Radiol 21, 2130-2138 (2011).

${ }^{12}$ J.G. Fletcher, et al., "Validation of Dual-Source Single-Tube Reconstruction as a Method to Obtain Half-Dose Images to Evaluate Radiation Dose and Noise Reduction: Phantom and Human Assessment Using CT Colonography and Sinogram-Affirmed Iterative Reconstruction (SAFIRE)," Journal of Computer Assisted Tomography 36, 560-569 510.1097/RCT.1090b1013e318263cc318261b (2012).

${ }^{13}$ C. von Falck, V. Bratanova, T. Rodt, B. Meyer, S. Waldeck, F. Wacker, and H.-o. Shin, "Influence of Sinogram Affirmed Iterative Reconstruction of CT Data on Image Noise Characteristics and Low-Contrast Detectability: An Objective Approach," PLoS ONE 8, e56875 (2013).

${ }^{14}$ A.N. Primak, J.C. Ramirez Giraldo, X. Liu, L. Yu, and C.H. McCollough, "Improved dual-energy material discrimination for dual-source $\mathrm{CT}$ by means of additional spectral filtration," Med Phys 36, 1359-1369 (2009).

${ }^{15}$ A.H. Mahnken, S. Stanzel, and B. Heismann, "Spectral rhoZ-projection method for characterization of body fluids in computed tomography: ex vivo experiments," Acad Radiol 16, 763-769 (2009). 



\section{CHAPTER}

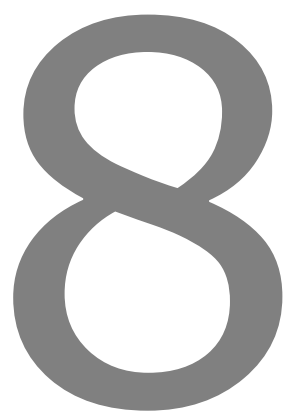

Deriving effective atomic numbers from DECT based on a parameterization of the ratio of high and low linear attenuation coefficients

Guillaume Landry, Joao Seco, Mathieu Gaudreault, Frank Verhaegen

Published in: Physics in Medicine and Biology 58:6851-6866, 2013. 


\section{ABSTRACT}

Introduction DECT can provide simultaneous estimation of relative electron density $\rho_{\mathrm{e}}$ and effective atomic number $Z_{\text {eff }}$. The ability to obtain these quantities $\left(\rho_{\mathrm{e}}, Z_{\text {eff }}\right)$ has been shown to benefit selected radiotherapy applications where tissue characterization is required. The conventional analysis method (spectral method) relies on knowledge of the CT scanner photon spectra which may be difficult to obtain accurately. We present an alternative approach based on a parameterization of the measured ratio of low and high $\mathrm{kVp}$ linear attenuation coefficients for deriving $Z_{\text {eff }}$ which does not require the estimation of the $\mathrm{CT}$ scanner spectra.

Materials and methods In a first approach, the tissue substitutes method (TSM), the Rutherford parameterization of the linear attenuation coefficients was employed to derive a relation between $Z_{\text {eff }}$ and the ratio of the linear attenuation coefficients measured at the low and high kVp of the CT scanner. A phantom containing 16 tissue mimicking inserts was scanned with a dual source DECT scanner at $80 \mathrm{kVp}$ and 140 $k V p$. The data from the 16 inserts phantom was used to obtain model parameters for the relation between $Z_{\text {eff }}$ and $\left.\mu\right|_{140 \mathrm{kvp}} ^{80 \mathrm{kvp}}$. The accuracy of the method was evaluated with a second phantom containing 4 tissue mimicking inserts.

The TSM was compared to a more complex approach, the reference tissues method (RTM), which requires the derivation of stoichiometric fit parameters. These were derived from the 16 inserts phantom scans and used to calculate CT numbers at $80 \mathrm{kVp}$ and $140 \mathrm{kVp}$ for a set of tabulated reference human tissues. Model parameters for the parameterization of $\left.\mu\right|_{140 \mathrm{kVp}} ^{80 \mathrm{kVp}}$ were estimated for this reference tissue dataset and compared to the results of the TSM.

Residuals on $Z_{\text {eff }}$ for the reference tissue dataset for both TSM and RTM were compared to those obtained from the spectral method.

Results The tissue substitutes were well fitted by the TSM with $R^{2}=0.9930$. Residuals on $Z_{\text {eff }}$ for the phantoms were similar between the TSM and spectral methods for $Z_{\text {eff }}<8$ while they were improved by the TSM for higher $Z_{\text {eff. }}$ The RTM fitted the reference tissue dataset well with $R^{2}=0.9999$. Comparing the $Z_{\text {eff }}$ extracted from TSM and the more complex RTM to the known values from the reference tissue dataset yielded errors of up to 0.3 and 0.15 units of $Z_{\text {eff }}$ respectively. The parameterization approach yielded standard deviations which were up to 0.3 units of $Z_{\text {eff }}$ higher than those observed with the spectral method for $Z_{\text {eff }}$ around 7.5.

Conclusion Procedures for the DECT estimation of $Z_{\text {eff }}$ removing the need for estimates of the CT scanner spectra have been presented. Both the TSM and the more complex RTM performed better than the spectral method. The RTM yielded the best results for the reference human tissue dataset reducing errors from up to 0.3 to 0.15 units of $Z_{\text {eff }}$ compared to the simpler TSM. Both TSM and RTM are simpler to implement than the spectral method which requires estimates of the CT scanner spectra. 


\section{INTRODUCTION}

Dual energy computed tomography (DECT), first discussed by Hounsfield ${ }^{1}$ and pioneered by Alvarez and Macovski ${ }^{2}$, is now available in clinical environments ${ }^{3,4}$. By acquiring images using a pair of $x$-ray spectra at well separated energies it is possible to derive the relative electron density $\rho_{\mathrm{e}}$ and effective atomic number $Z_{\text {eff }}$ of a substance. The work of Torikoshi et al. ${ }^{5}$, which relied on mono-energetic measurements, was extended by Bazalova et al. ${ }^{6}$ for the broad spectra typical of CT scanners in a similar fashion as Heismann et al. ${ }^{7}$ A series of articles showed that using $\rho_{\mathrm{e}}$ and $Z_{\text {eff }}$ benefits radiotherapy applications where tissue characterization in terms of density or composition is desirable $e^{6,-14}$. The method of Bazalova et al. requires estimates of the spectral distributions of the $x$-rays used for imaging. These estimates have been obtained by direct spectral measurements ${ }^{15}$ or, more commonly, by using predictions of the $x$-ray spectra validated by half value layer $(\mathrm{HVL})$ measurements ${ }^{12,16}$. The former method is cumbersome while the latter requires accurate knowledge of the CT scanner filtration. Due to the bowtie filter present in CT scanners as well as beam hardening in the object $\mathrm{x}$-ray spectra are not well defined. For these reasons Bazalova et al. recommended filtering the spectral distributions emitted by the $\mathrm{CT}$ scanner by a water thickness equivalent to the radius of the scanned object, to obtain the spectrum needed for the DECT method.

Saito proposed a calibration-based approach bypassing spectral knowledge for the measurement of $\rho_{\mathrm{e}}$ with $\mathrm{DECT}{ }^{17}$ based on the linearity of $\rho_{\mathrm{e}}$ with a weighted difference of the low and high kVp CT numbers. A fitting procedure was performed to find the optimal weighting factor.

In this work we investigate the use of a calibration procedure for the estimation of $Z_{\text {eff }}$ based on DECT scans of a widely used radiotherapy $\rho_{\mathrm{e}}$ calibration phantom. This approach is novel as it removes the need for CT scanner spectra estimation and subject attenuation, and thus facilitates the determination of $Z_{\text {eff }}$ for radiotherapy applications.

\section{MATERIALS AND METHODS}

\section{Relationship between $\mathrm{Z}_{\text {eff }}$ and the ratio of linear attenuation coefficients}

Rutherford et al. proposed the following parameterization for the linear attenuation coefficient $\mu$ of a material of density $\rho$ and single element $Z$ (with atomic mass $A$ ) as a function of energy $E$ :

$$
\mu=\rho N_{A} \frac{1}{A}\left[Z K^{\mathrm{KN}}(E)+Z^{n} K^{\mathrm{SCA}}(E)+Z^{m} K^{\mathrm{PE}}(E)\right],
$$


where $K^{\mathrm{KN}}$ is the Klein-Nishina coefficient ${ }^{18}$. The second term corresponds to the contributions of coherent scattering and the binding correction for incoherent scattering to attenuation while the third term corresponds to the photoelectric contribution to attenuation. The exponents $n$ and $m$ typically take the values 2.86 and 4.62, respectively. We make the assumption that this parameterization can be applied to a mixture by replacing $Z$ by a mixture specific non-integer effective atomic number $Z_{\text {eff }}$ and assuming that $(Z / A)_{\text {eff }}$ can be approximated by a constant for human tissues. We can thus write

$\mu_{\mathrm{kVp}} \approx \rho N_{\mathrm{A}}\left(A_{\mathrm{kVp}}+B_{\mathrm{kVp}} Z_{\mathrm{eff}}^{n-1}+C_{\mathrm{kVp}} Z_{\mathrm{eff}}^{m-1}\right)$,

where $\mu_{\mathrm{kVp}}$ corresponds to the average linear attenuation (weighted by the energy response) measured by a CT scanner operated at a certain $\mathrm{kVp}$. The constants $A_{\mathrm{kVp}}, B_{\mathrm{kVp}}$ and $C_{\mathrm{kVp}}$ correspond to the energy response weighted averages of the $K$ factors of equation 1 and are $k V p$ dependent. By taking the ratio of measured $\mu_{\mathrm{kVp}} / \mu_{\mathrm{kVp} \text {, water }}$ at a low (generally 80) and high (generally 140) kVp, defined as $\left.\mu\right|_{140 \mathrm{kVp}} ^{80 \mathrm{kVp}} \equiv\left(\mu_{80 \mathrm{kVp}} / \mu_{80 \mathrm{kVp}, \mathrm{water}}\right) /\left(\mu_{140 \mathrm{kVp}} / \mu_{140 \mathrm{kVp}, \text { water }}\right)$, we obtain:

$\left.\mu\right|_{140 \mathrm{kVp}} ^{80 \mathrm{kVp}} \approx \frac{A_{80 \mathrm{kVp}}+B_{80 \mathrm{kVp}} Z_{\mathrm{eff}}^{n-1}+C_{80 \mathrm{kVp}} Z_{\mathrm{eff}}^{m-1}}{A_{140 \mathrm{kVp}}+B_{140 \mathrm{kVp}} Z_{\mathrm{eff}}^{n-1}+C_{140 \mathrm{kVp}} Z_{\mathrm{eff}}^{m-1}}$,

where $1 / \mu_{\mathrm{kVp} \text {,water }}$ has been included in $A_{\mathrm{kVp}}, B_{\mathrm{kVp}}$ and $C_{\mathrm{kVp}}$. CT measurements $\left(\mathrm{HU}_{\mathrm{kVp}}\right)$ are related to $\mu_{\mathrm{kVp}} / \mu_{\mathrm{kVp} \text {, water }}$ by:

$\frac{\mu_{\mathrm{kVp}}}{\mu_{\text {water, kVp }}}=\mathrm{HU}_{\mathrm{kVp}} / 1000+1$.

We propose fitting Equation 8.3 to the measured $\left.\mu\right|_{140 \mathrm{kVp}} ^{80 \mathrm{kvp}}$ of a calibration phantom containing tissue mimicking materials for which elemental compositions are known. $Z_{\text {eff }}$ is generally defined as

$Z_{\text {eff }}=\sqrt[\beta]{\sum_{i}^{n} \frac{\omega_{i} Z_{i}}{A_{i}} Z_{i}^{\beta} / \sum_{i}^{n} \frac{\omega_{i} Z_{i}}{A_{i}}}$

where $i$ ranges over the $n$ elements composing a material and the weights $\omega_{i}$ correspond to the fractional mass of element $Z_{i}$ with atomic mass $A_{i}{ }^{19}$. The choice of $\beta$ will affect the value of $Z_{\text {eff }}$ and is further discussed below.

\section{Phantom measurements}

A Gammex RMI 467 (Gammex, Middleton, WI) electron density phantom was scanned at a dual source CT scanner (Siemens SOMATOM Definition FLASH, Siemens medical, Forchheim, Germany) operated in dual energy mode at $80 \mathrm{kVp}$ and $140 \mathrm{kVp}$. The 140 $k V p$ spectrum was filtered with a $\mathrm{Sn}$ filter $^{20}$. The CTDI ${ }_{\text {vol }}$ for the acquisition was 28.45 mGy (733 mAs at $80 \mathrm{kVp}$ and $233 \mathrm{mAs}$ at $140 \mathrm{kVp}$ ). Images were reconstructed with sinogram affirmed iterative reconstruction (SAFIRE) with a strength setting of 5 and 3 
$\mathrm{mm}$ slice thickness. It was verified that SAFIRE does not affect mean CT numbers in a region of interest (ROI) but reduces standard deviations in uniform materials, i.e. reduces imaging noise. The phantom loading pattern is illustrated in Figure 8.1.

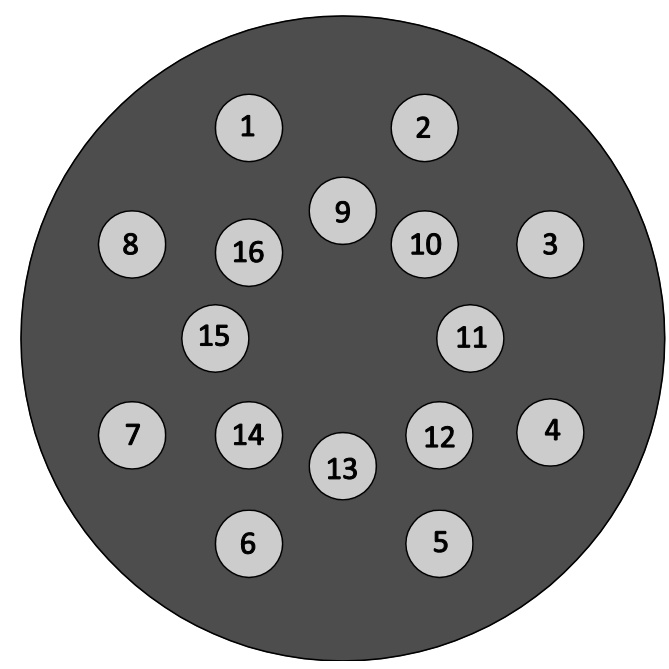

Figure 8.1: Insert loading pattern of the Gammex 467 phantom. Indices refer to the insert names of Table 8.3.

The Gammex 467 phantom CT images were imported in MATLAB (MathWorks, Inc., Natick, MA) where the mean and standard deviation of $\mathrm{HU}_{140 \mathrm{kVp},} \mathrm{HU}_{80 \mathrm{kVp}}$ and $\left.\mu\right|_{140 \mathrm{kVp}} ^{80 \mathrm{kVp}}$ were measured using ROls covering $90 \%$ of the uniform inserts in a central slice of the phantom.

A CIRS phantom (Model 002H5, CIRS Inc., Norfolk, VA) containing four inserts (lung, adipose, muscle and bone, product 002ED, CIRS Inc.) was scanned using the same settings (except CTDI $\mathrm{vol}=18 \mathrm{mGy}$ ). $\mathrm{HU}_{140 \mathrm{kVp},} \mathrm{HU}_{80 \mathrm{kVp}}$ and $\left.\mu\right|_{140 \mathrm{kVp}} ^{80 \mathrm{kvp}}$ were measured using ROIs covering $90 \%$ of the uniform inserts in a central slice of the phantom.

For both phantoms the compositions required to calculate $Z_{\text {eff }}$ were provided by manufacturers upon request and are presented in Tables 8.1 and 8.2. 
Table 8.1: Elemental weight fractions (\%) of the inserts of the Gammex 467 phantom, as well as their density $\rho$, electron density $\rho_{e}$ and effective atomic number $Z_{\text {eff }}$ calculated using $\beta=3.3$.

\begin{tabular}{|c|c|c|c|c|c|c|c|c|c|c|c|c|}
\hline $\begin{array}{l}\mathrm{Z} \\
\mathbf{A} \\
\end{array}$ & $\begin{array}{l}1 \\
1.01 \\
\end{array}$ & $\begin{array}{l}6 \\
12.01 \\
\end{array}$ & $\begin{array}{l}7 \\
14.01 \\
\end{array}$ & $\begin{array}{l}8 \\
15.99 \\
\end{array}$ & $\begin{array}{l}12 \\
24.3 \\
\end{array}$ & $\begin{array}{l}13 \\
26.98\end{array}$ & $\begin{array}{l}15 \\
30.97\end{array}$ & $\begin{array}{l}17 \\
35.45\end{array}$ & $\begin{array}{l}20 \\
40.08\end{array}$ & $\begin{array}{l}\rho \\
\mathrm{g} / \mathrm{cm}^{3}\end{array}$ & $\rho_{\mathrm{e}}$ & $Z_{\text {eff }}$ \\
\hline CT Solid Water & 8.00 & 67.3 & 2.39 & 19.87 & 0 & 0 & 0 & 0.14 & 2.31 & 1.022 & 0.992 & 7.74 \\
\hline LN-300 Lung & 8.46 & 59.38 & 1.96 & 18.14 & 11.19 & 0.78 & 0 & 0.10 & 0 & 0.3 & 0.292 & 7.55 \\
\hline LV1 Liver & 8.06 & 67.01 & 2.47 & 20.01 & 0 & 0 & 0 & 0.14 & 2.31 & 1.096 & 1.064 & 7.74 \\
\hline CB2-50\% CaCO3 & 4.77 & 41.63 & 1.52 & 32.00 & 0 & 0 & 0 & 0.08 & 20.02 & 1.559 & 1.469 & 12.54 \\
\hline B200 Bone Mineral & 6.65 & 55.52 & 1.98 & 23.64 & 0 & 0 & 3.24 & 0.11 & 8.87 & 1.152 & 1.103 & 10.42 \\
\hline SB3 Cortical Bone & 3.41 & 31.41 & 1.84 & 36.5 & 0 & 0 & 0 & 0.04 & 26.81 & 1.822 & 1.695 & 13.64 \\
\hline LN-450 Lung & 8.47 & 59.57 & 1.97 & 18.11 & 11.21 & 0.58 & 0 & 0.10 & 0 & 0.46 & 0.448 & 7.53 \\
\hline BRN-SR2 Brain & 10.83 & 72.54 & 1.69 & 14.86 & 0 & 0 & 0 & 0.08 & 0 & 1.051 & 1.047 & 6.09 \\
\hline AP6 Adipose & 9.06 & 72.3 & 2.25 & 16.27 & 0 & 0 & 0 & 0.13 & 0 & 0.947 & 0.928 & 6.21 \\
\hline IB Inner bone & 6.67 & 55.64 & 1.96 & 23.52 & 0 & 0 & 3.23 & 0.11 & 8.86 & 1.134 & 1.086 & 10.42 \\
\hline CB2-30\% $\mathrm{CaCO} 3$ & 6.68 & 53.48 & 2.12 & 25.61 & 0 & 0 & 0 & 0.11 & 12.01 & 1.331 & 1.276 & 10.90 \\
\hline Water Insert & 11.20 & 0 & 0 & 88.80 & 0 & 0 & 0 & 0 & 0 & 1.000 & 1.000 & 7.48 \\
\hline BR-12 Breast & 8.59 & 70.11 & 2.33 & 17.90 & 0 & 0 & 0 & 0.13 & 0.95 & 0.98 & 0.956 & 6.93 \\
\hline
\end{tabular}

Table 8.2: Elemental weight fractions (\%) of the inserts of the CIRS phantom as well as their density $\rho$, electron density $\rho_{e}$ and effective atomic number $Z_{\text {eff }}$ calculated using $\beta=3.3$.

\begin{tabular}{lllllllllllll}
\hline $\mathbf{Z}$ & $\mathbf{1}$ & $\mathbf{5}$ & $\mathbf{6}$ & $\mathbf{7}$ & $\mathbf{8}$ & $\mathbf{1 2}$ & $\mathbf{1 3}$ & $\mathbf{1 7}$ & $\mathbf{2 0}$ & $\boldsymbol{\rho}$ & $\rho_{\mathrm{e}}$ & $\mathbf{Z}_{\text {eff }}$ \\
$\mathbf{A}$ & $\mathbf{1 . 0 1}$ & $\mathbf{1 0 . 8 1}$ & $\mathbf{1 2 . 0 1}$ & $\mathbf{1 4 . 0 1}$ & $\mathbf{1 6}$ & $\mathbf{2 4 . 3}$ & $\mathbf{2 6 . 9 8}$ & $\mathbf{3 5 . 4 5}$ & $\mathbf{4 0 . 0 8}$ & $\mathbf{g} / \mathbf{c m}^{\mathbf{3}}$ & & \\
\hline lung (inhale) & 8.44 & 0 & 63.76 & 3.26 & 20.24 & 0 & 2.71 & 1.55 & 0 & 0.210 & 0.204 & 7.24 \\
average bone & 4.83 & 0 & 37.03 & 0.97 & 35.66 & 6.19 & 0 & 0.05 & 15.24 & 1.600 & 1.506 & 11.90 \\
muscle & 9.10 & 0 & 69.70 & 2.10 & 16.80 & 0 & 0 & 0.10 & 2.20 & 1.062 & 1.041 & 7.59 \\
plastic water-DT & 7.40 & 2.26 & 46.70 & 1.56 & 33.52 & 6.88 & 1.40 & 0.24 & 0 & 1.039 & 1.000 & 7.55 \\
adipose & 10.00 & 0 & 71.30 & 1.80 & 16.4 & 0 & 0 & 0.20 & 0.30 & 0.967 & 0.956 & 6.44 \\
\hline \hline
\end{tabular}

\section{Human tissue CT numbers}

In a second approach inspired from Schneider et al. ${ }^{21}$, the measurements of the Gammex RMI 467 phantom were used to derive the $k_{1, k v_{p}}$ and $k_{2, k v p}$ parameters ${ }^{22}$ of the stoichiometric fit procedure at $80 \mathrm{kVp}$ and $140 \mathrm{kVp}$. The parameters $k_{1}, \mathrm{kVp}$ and $k_{2, \mathrm{kVp}}$ are related to those of Equation 8.1 by:

$$
k_{1, \mathrm{kVp}}=\frac{\bar{K}^{\mathrm{SCA}}}{\bar{K}^{\mathrm{KN}}}, k_{2, \mathrm{kVp}}=\frac{\bar{K}^{\mathrm{PE}}}{\bar{K}^{\mathrm{KN}}},
$$

where the averaging is over the CT scanner spectral response at a given $\mathrm{kVp}$. The parameters were obtained by minimizing

$$
\sum_{\text {inserts }}\left[\frac{\mu_{\mathrm{kVp}}}{\mu_{\mathrm{kVp}, \text { water }}}\left(k_{1, \mathrm{kVp}}, k_{2, \mathrm{kVp}}\right)-\left(\frac{\mathrm{HU}_{\mathrm{kVp}}}{1000}+1\right)\right]^{2}
$$

with

$$
\frac{\mu_{\mathrm{kVp}}}{\mu_{\mathrm{kVp}, \text { water }}}\left(k_{1, \mathrm{kVp}}, k_{2, \mathrm{kVp}}\right)=\frac{\rho}{\rho_{\text {water }}} \frac{\left.\sum_{\mathrm{H}}^{n} w_{i=1} w_{i} / A_{i}\left(1+z_{i}+Z_{i}^{2.86} k_{1, \mathrm{kVp}}+k_{2, \mathrm{kVp}}\right)+w_{\mathrm{o}}^{4.62} k_{2, \mathrm{kVp}}\right)}{A_{\mathrm{O}}\left(8+8^{2.86} k_{1, \mathrm{kVp}}+8^{4.86} k_{2, \mathrm{kVp}}\right)},
$$

where the known elemental weights of the phantom inserts $w_{\mathrm{i}}$ and their mass densities $\rho$ are used. The Isqnonlin function of MATLAB's Optimization Toolbox was em- 
ployed to perform the minimization and initial values of the $k_{1, k v p}$ and $k_{2, k v p}$ parameters were set to $10^{-3}$ and $10^{-5}$ respectively for either values of $k V p$.

The parameters were used to generate a reference set of $\mathrm{HU}_{80 \mathrm{kV} p}, \mathrm{HU}_{140 \mathrm{kVp}}$ and $\left.\mu\right|_{140 \mathrm{kVp}} ^{80 \mathrm{kv}}$ for 73 human tissues using the reference compositions of Woodard and White ${ }^{23,24}$ and Equation 8.8. This list is the same as in Schneider et al. ${ }^{22}$ with the addition of two bladder tissues, filled and empty, and is the most recent data used in radiotherapy. This reference tissue dataset is investigated as the tissue mimicking plastics of the Gammex 467 phantom may not behave exactly as human tissue with respect to $Z_{\text {eff. }}$.

\section{Spectral method}

We implemented the method of Bazalova et al. ${ }^{6}$ to derive $Z_{\text {eff }}$ and $\rho_{\mathrm{e}}$ from $\mathrm{HU}_{80 \mathrm{kVp}}$ and $\mathrm{HU}_{140 \mathrm{kVp}}$ as described in the appendix of Landry et al. ${ }^{11}$ We made use of the HVLvalidated CT scanner spectra at $80 \mathrm{kVp}$ and $140 \mathrm{kVp}$, and the detector response described in Landry et al. ${ }^{12}$ These were estimated for the same CT scanner employed in this work. The spectra were filtered by an additional $16 \mathrm{~cm}$ of water as recommended in Bazalova et al. ${ }^{6}$ The method was applied to the images of the Gammex 467 and CIRS phantoms as well as the reference set of tissue $\mathrm{HU}_{80 \mathrm{kVp}}$ and $\mathrm{HU}_{140 \mathrm{kVp}}$.

\section{Evaluation of fitting procedure}

Two approaches were investigated in this work. In the simpler tissue substitutes method (TSM) model parameters for Equation 8.3 are obtained from fitting the Gammex 467 CT data. The more complex reference tissues method (RTM) makes use of model parameters derived form the reference tissue dataset generated with the stoichiometric calibration procedure described above. The steps involved in these two methods as well as the spectral method are summarized in Figure 8.2. For $\beta$ between 3 and $4, Z_{\text {eff }}$ was calculated with Equation 8.5 and fitted to Equation 8.3 using the fit function of the MATLAB curve fitting toolbox and $\left.\mu\right|_{140 \mathrm{kVp}} ^{80 \mathrm{kVp}}$ from the TSM and RTM. The fit parameter $A_{80 \mathrm{kVp}}$ was set to 1 . The remaining fit parameters $A_{\mathrm{kVp}}, B_{\mathrm{kVp}}$ and $C_{\mathrm{kVp}}$ were constrained to positive values. Initial estimates were calculated using the cross sections for water at the average energies of our estimates of the $80 \mathrm{kVp}$ and $140 \mathrm{kVp}$ spectra (52 keV and $93 \mathrm{keV}$ respectively) and a value of $Z_{\text {eff }}$ of 7.51 using $\beta=3.5$, taking into account that $A_{80 \mathrm{kV} p}$ was fixed at 1 . The exponents $m$ and $n$ were fixed at 4.62 and 2.86. For both the TSM and RTM the $\beta$ maximizing $R^{2}$ was determined. 
TSM

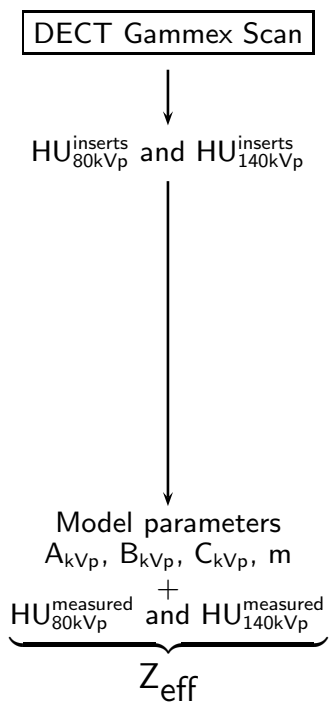

RTM

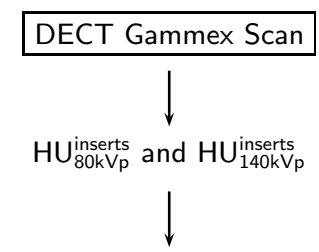

Stoichiometric parameters

$\mathrm{k}_{1, k \mathrm{p}_{\mathrm{p}}}$ and $\mathrm{k}_{2, \mathrm{k} \mathrm{v}_{\mathrm{p}}}$

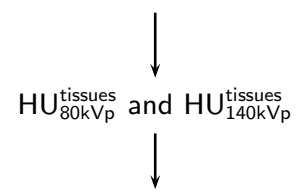

Model parameters

$A_{k V_{p}}, B_{k V_{p}}, C_{k V_{p}}, m$

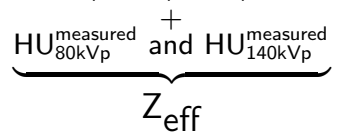

SPECTRAL METHOD
CT Scanner Spectra Detector response

NIST X-ray linear attenuation coefficients

Correction for attenuation through phantom

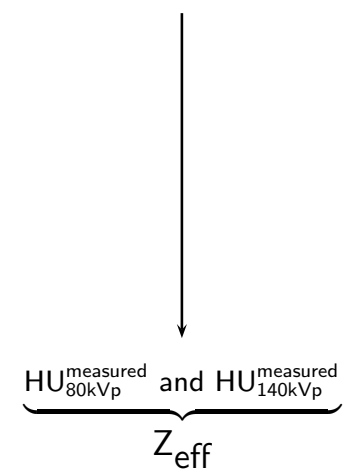

Figure 8.2: Summary of the three methods investigated in this work: the tissue substitute method (TSM), reference tissue method (RTM) and the spectral method. Items in boxes are required inputs. All methods are applied to measured CT numbers $\mathrm{HU}_{80 \mathrm{kVp}}^{\text {measured }}, \mathrm{HU}_{140 \mathrm{kVp}}^{\text {measured }}$ to yield an effective atomic number $Z_{\text {eff. }}$.

To avoid overfitting, the sensitivity of the fitting procedure to the parameters $A_{\mathrm{kVp}}$, $B_{\mathrm{kVp}}$ and $C_{\mathrm{kVp}}$ was investigated by sequentially setting fit parameters of terms with negligible contribution to zero and evaluating the residuals and confidence intervals for both the TSM and RTM. Additionally, a variant of equation (3) where $B_{\mathrm{kVp}}=0$ and $m$ was a variable was investigated as it provides a closed form for the inverse function $f$ :

$$
\begin{aligned}
& \left.\mu\right|_{140 \mathrm{kVp}} ^{80 \mathrm{kVp}} \rightarrow Z_{\mathrm{eff}}: \\
& \left.\mu\right|_{140 \mathrm{kVp}} ^{80 \mathrm{kVp}} \approx \frac{A_{80 \mathrm{kVp}}+C_{80 \mathrm{kVp}} Z_{\mathrm{eff}}^{m-1}}{A_{140 \mathrm{kVp}}+C_{140 \mathrm{kVp}} Z_{\mathrm{eff}}^{m-1}} .
\end{aligned}
$$

Equation 8.9 is equivalent to Equation 17 in Heismann et al. ${ }^{7}$ 


\section{Comparison with spectral method}

The residuals on $Z_{\text {eff }}$ of the TSM and RTM were evaluated by numerically inverting Equation 8.3 or Equation 8.9. The results of these two methods were compared to the $Z_{\text {eff }}$ obtained from the spectral approach described above. Two $Z_{\text {eff }}$ comparisons with reference values were performed to evaluate accuracy; TSM vs. the spectral method using the phantom data and TSM/RTM vs. the spectral method using the reference tissue dataset. The comparison using the reference tissue dataset was considered the most important test.

The noise levels resulting from the TSM/RTM were compared to those from the spectral method by calculating a $Z_{\text {eff }}$ map of the Gammex 467 phantom for each method and measuring the standard deviation in each insert.

\section{RESULTS AND DISCUSSION}

Table 8.3 presents the mean and standard deviations of the measured CT numbers for each insert of the Gammex 467 phantom as well as $\left.\mu\right|_{140 \mathrm{kVp}} ^{80 \mathrm{kvp}}$. Refer to Figure 8.1 for the insert loading configuration used for scanning. We verified that the use of the SAFIRE iterative reconstruction with strength 5 reduced the standard deviations on $\mathrm{HU}_{80 \mathrm{kVp}}$ and $\mathrm{HU}_{140 \mathrm{kVp}}$ by about a factor 2 when compared to filtered backprojection without altering mean values. Table 8.4 presents similar data for the CIRS phantom. 
Table 8.3: Measured CT numbers (mean HU \pm standard deviation) at $140 \mathrm{kVp}, 80 \mathrm{kVp}$ and $\left.\mu\right|_{140 \mathrm{kVp}} ^{80 \mathrm{kv}}$ of the inserts of the Gammex 467 phantom scanned in the configuration of Figure 8.1. Inserts ordered by increasing density. ID 17 corresponds to a solid water (same material as phantom) ROI in the centre of the phantom while ID 18 to a solid water ROI at the periphery.

\begin{tabular}{|c|c|c|c|c|}
\hline insert ID & material & $\mathrm{HU}_{140 \mathrm{kVp}}$ & $H U_{80 k V p}$ & $\left.\mu\right|_{140 \mathrm{kVp}} ^{80 \mathrm{kVp}}$ \\
\hline 2 & LN-300 Lung & $-708 \pm 12$ & $-700 \pm 15$ & $1.028 \pm 0.067$ \\
\hline 9 & LN-450 Lung & $-549 \pm 8$ & $-540 \pm 11$ & $1.020 \pm 0.031$ \\
\hline 11 & AP6 Adipose & $-78 \pm 8$ & $-110 \pm 12$ & $0.966 \pm 0.015$ \\
\hline 16 & BR-12 Breast & $-44 \pm 10$ & $-64 \pm 12$ & $0.979 \pm 0.016$ \\
\hline 15 & Water Insert & $-0 \pm 9$ & $-10 \pm 14$ & $0.990 \pm 0.017$ \\
\hline 1 & CT Solid Water & $3 \pm 6$ & $11 \pm 9$ & $1.008 \pm 0.011$ \\
\hline 3 & CT Solid Water & $4 \pm 7$ & $12 \pm 10$ & $1.008 \pm 0.012$ \\
\hline 5 & CT Solid Water & $2 \pm 7$ & $10 \pm 11$ & $1.008 \pm 0.013$ \\
\hline 13 & CT Solid Water & $-1 \pm 9$ & $3 \pm 12$ & $1.004 \pm 0.015$ \\
\hline 17 & CT Solid Water & $6 \pm 6$ & $10 \pm 8$ & $1.004 \pm 0.010$ \\
\hline 18 & CT Solid Water & $-2 \pm 12$ & $6 \pm 16$ & $1.007 \pm 0.020$ \\
\hline 10 & BRN-SR2 Brain & $35 \pm 9$ & $2 \pm 12$ & $0.968 \pm 0.014$ \\
\hline 4 & LV1 Liver & $77 \pm 7$ & $86 \pm 11$ & $1.009 \pm 0.012$ \\
\hline 12 & IB Inner bone & $152 \pm 9$ & $285 \pm 15$ & $1.116 \pm 0.015$ \\
\hline 7 & B200 Bone Mineral & $174 \pm 8$ & $309 \pm 12$ & $1.115 \pm 0.012$ \\
\hline 14 & CB2-30\% CaCO3 & $370 \pm 12$ & $565 \pm 20$ & $1.142 \pm 0.018$ \\
\hline 6 & $\mathrm{CB} 2-50 \% \mathrm{CaCO} 3$ & $663 \pm 10$ & $1067 \pm 17$ & $1.243 \pm 0.013$ \\
\hline 8 & SB3 Cortical Bone & $1000 \pm 12$ & $1658 \pm 26$ & $1.329 \pm 0.015$ \\
\hline
\end{tabular}

Table 8.4: Measured CT numbers (mean HU \pm standard deviation) at $140 \mathrm{kVp}, 80 \mathrm{kVp}$ and $\left.\mu\right|_{140 \mathrm{kVp}} ^{80 \mathrm{kV}}$ of the inserts of the CIRS phantom. Inserts ordered by increasing density.

\begin{tabular}{cccc}
\hline \hline material & $\mathbf{H U}_{140 \mathrm{kV} \mathbf{p}}$ & $\mathbf{H U}_{80 \mathrm{kVp}}$ & $\left.\mu\right|_{140 \mathrm{kVp}} ^{80 \mathrm{kV}}$ \\
\hline lung (inhale) & $-787 \pm 7$ & $-786 \pm 8$ & $1.005 \pm 0.049$ \\
adipose & $-61 \pm 5$ & $-95 \pm 8$ & $0.964 \pm 0.010$ \\
plastic water-DT & $-6 \pm 6$ & $-6 \pm 8$ & $1.001 \pm 0.010$ \\
muscle & $33 \pm 12$ & $33 \pm 13$ & $1.000 \pm 0.018$ \\
average bone & $671 \pm 5$ & $1037 \pm 12$ & $1.219 \pm 0.008$ \\
\hline \hline
\end{tabular}

Table 8.5: Results of the stoichiometric fit procedure for the Gammex 467 phantom

\begin{tabular}{lcc}
\hline \hline & $\boldsymbol{k}_{1, \mathrm{kvp}}$ & $\boldsymbol{k}_{2, \mathrm{kvp}}$ \\
\hline $80 \mathrm{kVp}$ & $5.168 \mathrm{E}-04$ & $4.649 \mathrm{E}-05$ \\
$140 \mathrm{kVp}$ & $5.000 \mathrm{E}-05$ & $1.445 \mathrm{E}-05$ \\
\hline \hline
\end{tabular}

The results of the stoichiometric calibration procedure are in Table 8.5. The largest residual error on $\mathrm{HU}_{80 \mathrm{kVp}}\left(\mathrm{HU}_{140 \mathrm{kVp}}\right)$ for the calibration phantom (Gammex 467) was 12 $\mathrm{HU}(8 \mathrm{HU})$, which is of the same order as the uncertainty on measured HU (see Table 8.3). 


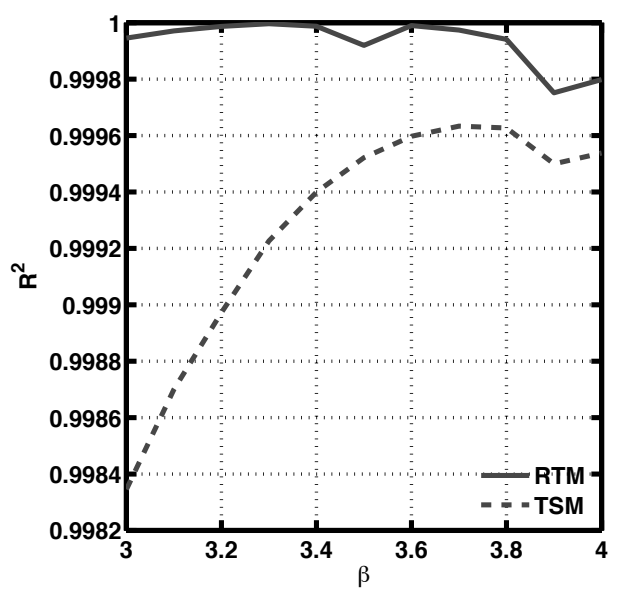

Figure 8.3: $R^{2}$ as a function of $\beta$ for the reference tissue data set and the Gammex 467 phantom fitted to Equation 8.3.

Figure 8.3 shows the behaviour of $R^{2}$ where the data of the Gammex 467 phantom (TSM) and the reference tissue dataset (RTM) were fitted with Equation 8.3 and $\beta$ varied between 3 and 4 . When performing the fitting it was observed that the lung inserts exhibited the largest residuals and dominated the behaviour of $R^{2}$ as a function of $\beta$, they were thus ignored in the fitting of Equation 8.3 to determine $\beta$ (further discussed below). A similar observation was made for the thyroid in the reference tissue dataset. The thyroid contains a relatively large amount of iodine and thus behaves differently than other tissues and was also ignored. From Figure 8.3 we see that the maximum $R^{2}$ for the Gammex 467 phantom occurred for $\beta=3.7$. For the tissues the maximum was at $\beta=3$.3. For ease of comparison we have decided to use the exponent value of $\beta=3.3$ as also recommended by Yang et al. ${ }^{10}$ and Landry et al. ${ }^{11}$ when using the spectral method. This approximation is further justified as the residuals do not vary significantly when $\beta$ is changed. From now on $Z_{\text {eff }}$ will refer to the result of Equation 8.5 using $\beta=3.3$.

Equation 8.3 was investigated to determine the relevance of the parameters $A_{\mathrm{kvp}}$, $B_{\mathrm{kVp}}$ and $C_{\mathrm{kVp}}$ for both the Gammex 467 phantom and the reference tissues. The values of the parameters are presented in Table 8.6. It has been observed from these results that the photoelectric term at high energy, $\mathrm{C}_{140 \mathrm{kvp}}$, can be omitted. The fitting procedure has been repeated with this condition. The values of the resulting parameters are also shown in Table 8.6. The sum of squared difference and the $R^{2}$ obtained with the two equations are almost identical to each other as applied to either Gammex 467 or the reference tissues. 
Table 8.6: Fit parameters for Equation 8.3.

\begin{tabular}{lllll}
\hline \hline & Equation (3) & & \multicolumn{2}{c}{ Equation (3) with $C_{140 \mathrm{kvp}=0}$} \\
& Gammex 467 & Reference tissues & Gammex 467 & Reference tissues \\
\hline $\boldsymbol{A}_{\mathbf{8 0 \mathrm { kVp }}}$ & 1 & 1 & 1 & 1 \\
$\boldsymbol{B}_{\mathbf{8 0 \mathrm { kvp }}}$ & 0.001916 & 0.001194 & 0.001924 & 0.01118 \\
$\boldsymbol{C}_{80 \mathrm{kVp}}$ & $1.707 \mathrm{E}-05$ & $1.083 \mathrm{E}-04$ & $1.701 \mathrm{E}-05$ & $1.016 \mathrm{E}-04$ \\
$\boldsymbol{A}_{140 \mathrm{kVp}}$ & 1.104 & 1.026 & 1.104 & 1.031 \\
$\boldsymbol{B}_{140 \mathrm{kVp}}$ & $2.109 \mathrm{E}-10$ & 0.01505 & $7.747 \mathrm{E}-12$ & 0.01393 \\
$\boldsymbol{C}_{140 \mathrm{kVp}}$ & $1.777 \mathrm{E}-10$ & $2.696 \mathrm{E}-08$ & 0 & 0 \\
\hline \hline
\end{tabular}

Another trial was performed in order to reduce the parameters of Equation 8.3 by setting to zero the coherent scattering term $B_{\mathrm{k} V \mathrm{p}}$ for the two energies (Equation 8.9). The results of the fitting procedure showed that the accuracy of the fit is not degraded while the confidence intervals is greatly reduced. The exponent $m$ was chosen to be a parameter as well in the fitting procedure. The parameters obtained with this choice are presented in Table 8.7. The resulting equation can be inverted allowing a direct determination of $Z_{\text {eff }}$ given the values of $\mathrm{HU}_{80 \mathrm{kVp}}$ and $\mathrm{HU}_{140 \mathrm{kVp}}$ as opposed to the other two equations above. Because of the practical use of this equation, we used it in the rest of this article. However, all conclusions drawn for this choice also apply for the other two equations discussed above.

Table 7. Fit parameters for the Gammex 467 phantom and the reference tissues based on Equation 8.9.

\begin{tabular}{llll}
\hline \hline & Gammex 467 & Gammex 467 excl. lung & reference tissues \\
\hline $\boldsymbol{A}_{\text {8okvp }}$ & 1 & 1 & 1 \\
$\boldsymbol{B}_{\text {8okvp }}$ & 0 & 0 & 0 \\
$\boldsymbol{C}_{\text {80kvp }}$ & 0.0002249 & 0.00015428 & 0.00013166 \\
$\boldsymbol{A}_{140 \mathrm{kVp}}$ & 1.0748 & 1.0743 & 1.0668 \\
$\boldsymbol{B}_{140 \mathrm{kVp}}$ & 0 & 0 & 0 \\
$\boldsymbol{C}_{140 \mathrm{kVp}}$ & $4.31 \mathrm{E}-05$ & $4.93 \mathrm{E}-05$ & $4.27 \mathrm{E}-05$ \\
$\boldsymbol{m}$ & 4.0008 & 4.2429 & 4.2961 \\
\hline \hline
\end{tabular}

Figure 8.4 presents the results of the fitting procedure for the Gammex 467 phantom using Equation 8.9. The fitting procedure yielded $R^{2}=0.9930$; the $95 \%$ confidence intervals are also plotted in Figure 8.4. Equation 8.9 appears to capture the behaviour of $Z_{\text {eff }}$ vs. $\left.\mu\right|_{140 \mathrm{kVp}} ^{80 \mathrm{kvp}}$ well. The two data points with the largest residuals are the LN-300 and LN-450 lung inserts. Table 8.3 shows that these two inserts have significantly higher standard deviations for $\left.\mu\right|_{140 \mathrm{kVp}} ^{80 \mathrm{kVp}}$ because of their low CT numbers. When excluding the lung inserts from the fitting procedure, $R^{2}$ was 0.9992 . Excluding the lung 
inserts caused the fitted curve to be slightly lower for $Z_{\text {eff }}<8$ than the one obtained when the lung inserts included. These inserts have a $Z_{\text {eff }}$ very similar to the water insert (7.55 for LN-300 and LN-450 vs. 7.48 for water). Given the uncertainty on the lung inserts ( 4 times higher than water, see table 3 ) and their impact on the fitting procedure it is questionable whether they should be included and it can be argued that a measurement of the water insert is sufficient.
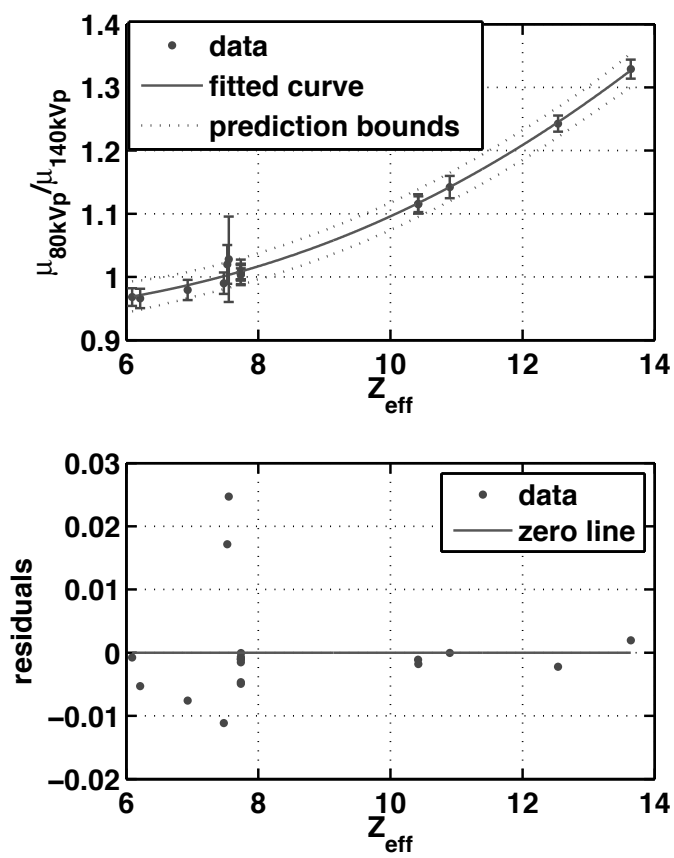

Figure 8.4: Result of fitting procedure for the Gammex 467 phantom with Equation 8.9 (TSM). Error bars represent standard deviation. Top Data and fitted curve, as well as $95 \%$ prediction bounds. Bottom Residuals. $R^{2}=0.9930$.

Figure 8.5 presents the $Z_{\text {eff }}$ errors (measurement - reference) for the Gammex 467 and CIRS phantoms. $Z_{\text {eff }}$ was calculated from $\mathrm{HU}_{80 \mathrm{kVp}}$ and $\mathrm{HU}_{140 \mathrm{kVp}}$ images using the spectral method and using the parameters from the TSM excluding lung inserts. For each insert of each phantom the mean value and standard deviation of $Z_{\text {eff }}$ was calculated in ROIs. For the Gammex 467 phantom we saw a trend of underestimation for $Z_{\text {eff }}>7.5$ when using the spectral method. This trend is not present with the TSM. As expected from the residuals of Figure 8.4, the lung inserts prove again problematic. Besides the lung inserts, we see that errors of the order of -0.3 units of $Z_{\text {eff }}$ are present. The errors on the CIRS phantom when using the spectral method appear to follow a similar trend as those of the Gammex 467 phantom. For the CIRS phantom it appears the TSM is reversing this trend. It is possible that a phantom size effect is present and that calibra- 
tion should be body part specific. The Gammex 467 phantom is $33 \mathrm{~cm}$ in diameter while the CIRS phantom is elliptical with a thickness of $20 \mathrm{~cm}$ and a width of $30 \mathrm{~cm}$. Figure 8.6 shows $Z_{\text {eff }}$ images of both phantoms obtained from the TSM and spectral methods. Qualitatively the TSM yields a higher intensity in high $Z_{\text {eff }}$ materials, as well as slightly higher noise levels.

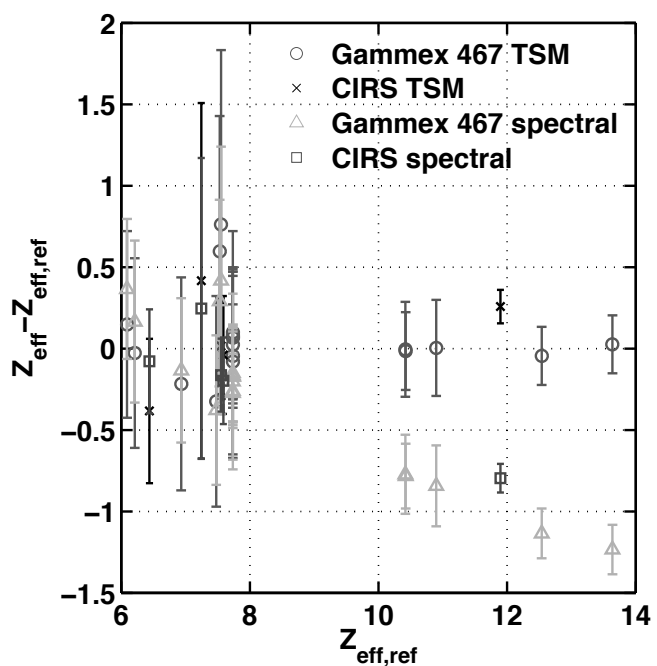

Figure 8.5: $Z_{\text {eff }}$ residuals for the Gammex 467 and CIRS phantoms. $Z_{\text {eff }}$ has been calculated using both spectral approach and the TSM for each phantom. We used the fit obtained excluding the lung inserts. Error bars represent standard deviation in ROls covering the inserts in a central slice of each phantom. 


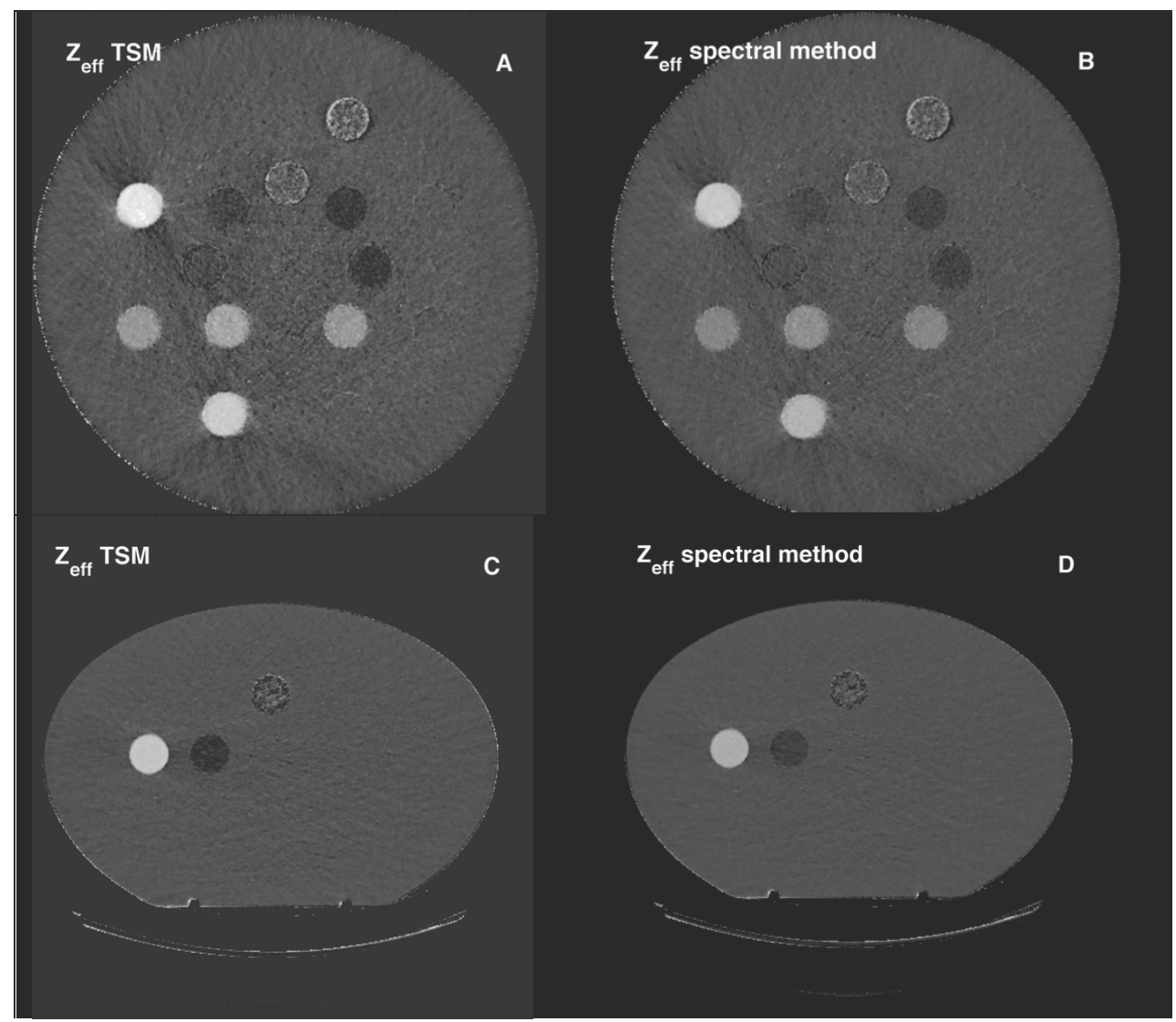

Figure 8.6: $Z_{\text {eff }}$ images of the $(A, B)$ Gammex 467 phantom from the (A) TSM and (B) spectral method. $(C, D)$ images of the CIRS phantom. Grayscale range of $Z_{\text {eff }}$ from 5 to 14 . All images at same scale.

Figure 8.7 presents the result of the RTM which has been calculated using the stoichiometric fit parameters of Table 8.5. Here $R^{2}=0.9999$, the largest residual is for the thyroid, which contains iodine. The fit parameters corresponding to the curves of Figures 8.4 and 8.7 as well as those from excluding the lung inserts are presented in Table 8.7. Figure 8.8 plots the three fits (phantom including lung, phantom excluding lung, and tissues) as well as the data points used to obtain the fits for $Z_{\text {eff }}<8$ where differences are the largest. We observe that there is a difference between the data points of the tissue mimicking inserts of the phantoms and the reference tissues. When including the lung inserts in the TSM the curve is shifted upwards, while excluding them gives a curve which is more different than the RTM. The data shown in Figure 8.7 suggest that it is likely necessary to adopt the RTM as the composition of the phantom inserts may not perfectly mimic the behaviour of human tissues with respect to $Z_{\text {eff. }}$. 

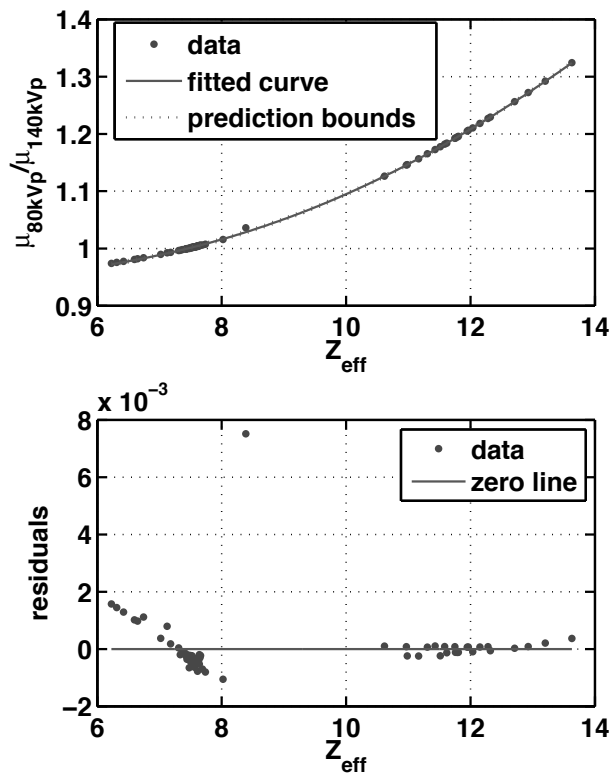

Figure 8.7: Result of fitting procedure for the reference tissues (RTM). Top Data and fitted curve, as well as $95 \%$ prediction bounds (overlapping the curve). Bottom Residuals. $R^{2}=0.9999$.

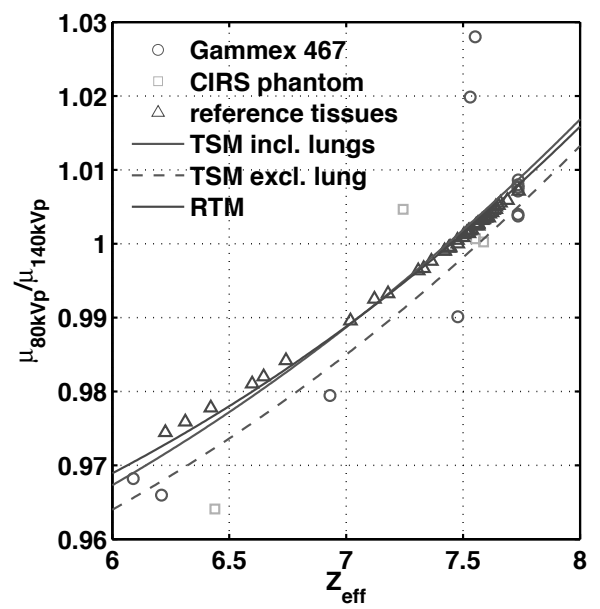

Figure 8.8: Difference between fitted curves for the Gammex 467 phantom and the reference tissues for TSM with and without the lung inserts and RTM. Green data points are for the second phantom used in this study. 
Figure 8.9 plots the $Z_{\text {eff }}$ residuals for the reference tissues (measurement - reference) where $Z_{\text {eff }}$ has been calculated with the spectral method, the TSM and the RTM. There are two TSM fits; including and excluding the lung inserts. When using the spectral method the same error trend as observed in Figure 8.5 is present. The RTM mitigates this trend and brings errors well within 0.2 units of $Z_{\text {eff }}$ when using the tissue fit. For the TSM with the lung inserts excluded, the residuals are higher due to the difference between the phantom and tissue curves (Figure 8.8).

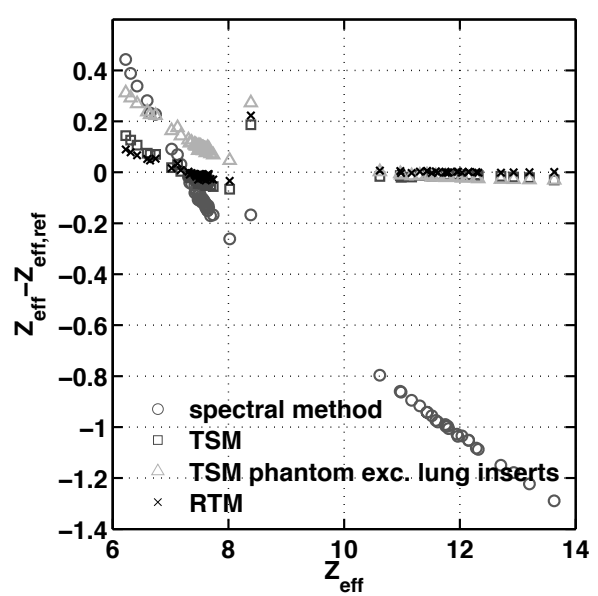

Figure 8.9: $Z_{\text {eff }}$ residuals for the reference tissues for the spectral method and the fitting method. 
Table 8.8: Measured $Z_{\text {eff }}$ from the fitting $\left(Z_{\text {effffitting }}\right)$ with and without the lung inserts and spectral $\left(Z_{\text {eff,spectral }}\right)$ procedures for each insert of the Gammex 467 phantom (mean \pm standard deviation) compared to the reference $Z_{\text {eff,ref. }}$ Insert numbers correspond to those of Figure 8.1 and Table 8.3.

\begin{tabular}{lcccc}
\hline \hline insert ID & $\boldsymbol{Z}_{\text {eff,ref }}$ & $\mathbf{Z}_{\text {eff,fitting }}$ & $\mathbf{Z}_{\text {eff,fiting }}$ excl. lung & $\mathbf{Z}_{\text {eff,spectral }}$ \\
\hline 2 & 7.55 & $8.2 \pm 1.1$ & $8.3 \pm 1.1$ & $8.0 \pm 0.8$ \\
9 & 7.53 & $8.0 \pm 0.9$ & $8.1 \pm 0.8$ & $7.8 \pm 0.6$ \\
11 & 6.21 & $6.1 \pm 0.5$ & $6.2 \pm 0.6$ & $6.4 \pm 0.5$ \\
16 & 6.93 & $6.6 \pm 0.7$ & $6.7 \pm 0.7$ & $6.8 \pm 0.4$ \\
15 & 7.48 & $7.0 \pm 0.7$ & $7.2 \pm 0.6$ & $7.1 \pm 0.5$ \\
1 & 7.74 & $7.7 \pm 0.4$ & $7.8 \pm 0.4$ & $7.6 \pm 0.3$ \\
3 & 7.74 & $7.7 \pm 0.4$ & $7.8 \pm 0.4$ & $7.6 \pm 0.3$ \\
5 & 7.74 & $7.7 \pm 0.4$ & $7.8 \pm 0.4$ & $7.6 \pm 0.3$ \\
13 & 7.74 & $7.5 \pm 0.6$ & $7.7 \pm 0.6$ & $7.5 \pm 0.4$ \\
17 & 7.74 & $7.6 \pm 0.3$ & $7.7 \pm 0.3$ & $7.5 \pm 0.2$ \\
18 & 7.74 & $7.6 \pm 0.7$ & $7.8 \pm 0.7$ & $7.5 \pm 0.5$ \\
10 & 6.09 & $6.1 \pm 0.6$ & $6.2 \pm 0.6$ & $6.5 \pm 0.4$ \\
4 & 7.74 & $7.7 \pm 0.4$ & $7.8 \pm 0.4$ & $7.6 \pm 0.3$ \\
12 & 10.42 & $10.4 \pm 0.3$ & $10.4 \pm 0.3$ & $9.6 \pm 0.2$ \\
7 & 10.42 & $10.4 \pm 0.2$ & $10.4 \pm 0.2$ & $9.6 \pm 0.2$ \\
14 & 10.9 & $10.9 \pm 0.3$ & $10.9 \pm 0.3$ & $10.1 \pm 0.2$ \\
6 & 12.54 & $12.5 \pm 0.2$ & $12.5 \pm 0.2$ & $11.4 \pm 0.2$ \\
8 & 13.64 & $13.7 \pm 0.2$ & $13.7 \pm 0.2$ & $12.4 \pm 0.2$ \\
\hline \hline
\end{tabular}

Table 8.8 presents the mean and standard deviations of $Z_{\text {eff }}$ for each insert of the Gammex 467 phantom for TSM (including and excluding lung inserts) and spectral methods. The standard deviations of the TSM are generally higher by about 0.1 to 0.3 units of $Z_{\text {eff }}$ than those of the spectral method for $Z_{\text {eff }}$ around 7, where the slope of the $\left.Z_{\text {eff }} \rightarrow \mu\right|_{140 \mathrm{kvp}} ^{80 \mathrm{kvp}}$ relation is shallower. For the higher values of $Z_{\mathrm{eff}}(>9)$ the two methods yield similar standard deviations. Inserts $1,3,5,13,17$ and 18 are solid water positioned at different locations in the phantom and can be used to assess the uncertainties associated with beam hardening variations in the phantom. We observe that the mean values in the different inserts vary from $7.5 \pm 0.6$ to $7.7 \pm 0.4$ for the TSM (including lung inserts) and from $7.5 \pm 0.4$ to $7.6 \pm 0.3$ for the spectral method. Similar standard deviations are expected from the RTM.

\section{CONCLUSION}

We have presented methods where a DECT scan of the Gammex RMI 467 scan is used to obtain a calibration of $Z_{\text {eff }}$ Vs. $\left.\mu\right|_{140 \mathrm{kVp}} ^{80 \mathrm{kVp}}$ using a functional form derived from the parameterization of Rutherford et al. For the Gammex 467 phantom the errors on $Z_{\text {eff }}$ are at most 0.3 units of $Z_{\text {eff }}$ with TSM compared to errors of up to -1.2 units of $Z_{\text {eff }}$ with the spectral method. When applied to reference tissues generated with the stoichiometric fit procedure (RTM) it was found that the accuracy on $Z_{\text {eff }}$ was superior to that from the spectral method, with residuals smaller than 0.15 units of $Z_{\text {eff. }}$ From the 
Gammex 467 phantom it was observed that the RTM produces higher noise levels than the spectral method (standard deviation of ROls covering uniform inserts). Given the uncertainty of $\left.\mu\right|_{140 \mathrm{kVp}} ^{80 \mathrm{kVp}}$ for the lung inserts of the Gammex 467 phantom we recommend excluding them from the TSM. As the fitted curves for the TSM and RTM differ for $Z_{\text {eff }}<8$ we recommend employing the RTM.

\section{ACKNOWLEDGEMENTS}

Dr Joachim E. Wildberger is acknowledged for providing access to the DECT scanner. We thank Dr. Bernhard Schmidt from Siemens Medical for providing information on the operation of the DECT scanner. Frank P. Eschbach and Jasper P. Jansen are acknowledged for adapting scripts to analyse the Gammex 467 phantom measurements. We acknowledge Gammex RMI and Vladimir Varchena of CIRS for divulging phantom compositions. G.L. would like to acknowledge the O'Brien Foundation for funding. Patrick V. Granton is acknowledged for commenting on the manuscript.

\section{REFERENCES}

${ }^{1}$ G.N. Hounsfield, "Computerized transverse axial scanning (tomography): Part 1. Description of system," British Journal of Radiology 46, 1016-1022 (1973).

${ }^{2}$ R.E. Alvarez and A. Macovski, "Energy-selective reconstructions in X-ray computerized tomography," Phys Med Biol 21, 733-744 (1976).

${ }^{3}$ T.G. Flohr, et al., "Dual-source spiral CT with pitch up to 3.2 and $75 \mathrm{~ms}$ temporal resolution: image reconstruction and assessment of image quality," Med Phys 36, 5641-5653 (2009).

${ }^{4}$ M.M. Goodsitt, E.G. Christodoulou, and S.C. Larson, "Accuracies of the synthesized monochromatic CT numbers and effective atomic numbers obtained with a rapid $\mathrm{kVp}$ switching dual energy CT scanner," Med Phys 38, 2222-2232 (2011).

${ }^{5} \mathrm{M}$. Torikoshi, et al., "Electron density measurement with dual-energy $\mathrm{x}$-ray CT using synchrotron radiation," Phys Med Biol 48, 673-685 (2003).

${ }^{6} \mathrm{M}$. Bazalova, J.F. Carrier, L. Beaulieu, and F. Verhaegen, "Dual-energy CT-based material extraction for tissue segmentation in Monte Carlo dose calculations," Phys Med Biol 53, 2439-2456 (2008).

${ }^{7}$ B.J. Heismann, J. Leppert, and K. Stierstorfer, "Density and atomic number measurements with spectral x-ray attenuation method," Journal of Applied Physics 94, 2073-2079 (2003).

${ }^{8}$ J.F. Williamson, S. Li, S. Devic, B.R. Whiting, and F.A. Lerma, "On two-parameter models of photon cross sections: application to dual-energy CT imaging," Med Phys 33, 4115-4129 (2006).

${ }^{9}$ B. Heismann and M. Balda, "Quantitative image-based spectral reconstruction for computed tomography," Med Phys 36, 4471-4485 (2009).

${ }^{10} \mathrm{M}$. Yang, G. Virshup, J. Clayton, X.R. Zhu, R. Mohan, and L. Dong, "Theoretical variance analysis of single- and dual-energy computed tomography methods for calculating proton stopping power ratios of biological tissues," Phys Med Biol 55, 1343-1362 (2010). 
${ }^{11}$ G. Landry, P.V. Granton, B. Reniers, M.C. Ollers, L. Beaulieu, J.E. Wildberger, and F. Verhaegen, "Simulation study on potential accuracy gains from dual energy CT tissue segmentation for low-energy brachytherapy Monte Carlo dose calculations," Physics in medicine and biology 56, 6257-6278 (2011).

${ }^{12}$ G. Landry, B. Reniers, P.V. Granton, B. van Rooijen, L. Beaulieu, J.E. Wildberger, and F. Verhaegen, "Extracting atomic numbers and electron densities from a dual source dual energy CT scanner: Experiments and a simulation model," Radiotherapy and Oncology 100, 375-379 (2011).

${ }^{13}$ M. Yang, Dual Energy Computed Tomography for Proton Therapy Treatment Planning, in Graduate School of Biomedical Sciences. 2011, The University of Texas: Houston.

${ }^{14}$ A. Malusek, M. Karlsson, M. Magnusson, and G.A. Carlsson, "The potential of dual-energy computed tomography for quantitative decomposition of soft tissues to water, protein and lipid in brachytherapy," Phys Med Biol 58, 771-785 (2013).

${ }^{15} \mathrm{M}$. Bazalova and F. Verhaegen, "Monte Carlo simulation of a computed tomography $\mathrm{x}$-ray tube," Phys Med Biol 52, 5945-5955 (2007).

${ }^{16}$ R.L. Kruger, C.H. McCollough, and F.E. Zink, "Measurement of half-value layer in x-ray CT: a comparison of two noninvasive techniques," Med Phys 27, 1915-1919 (2000).

${ }^{17} \mathrm{M}$. Saito, "Potential of dual-energy subtraction for converting CT numbers to electron density based on a single linear relationship," Med Phys 39, 2021-2030 (2012).

${ }^{18}$ R.A. Rutherford, B.R. Pullan, and I. Isherwood, "Measurement of effective atomic number and electron density using an EMI scanner," Neuroradiology 11, 15-21 (1976).

${ }^{19}$ H.E. Johns and J.R. Cunningham, The physics of radiology. 4th ed. 1983, Springfield, III., U.S.A.: Charles C. Thomas. xix, $796 \mathrm{p}$.

${ }^{20}$ A.N. Primak, J.C. Ramirez Giraldo, X. Liu, L. Yu, and C.H. McCollough, "Improved dual-energy material discrimination for dual-source $\mathrm{CT}$ by means of additional spectral filtration," Med Phys 36, 1359-1369 (2009).

${ }^{21}$ U. Schneider, E. Pedroni, and A. Lomax, "The calibration of CT Hounsfield units for radiotherapy treatment planning," Phys Med Biol 41, 111-124 (1996).

${ }^{22}$ W. Schneider, T. Bortfeld, and W. Schlegel, "Correlation between CT numbers and tissue parameters needed for Monte Carlo simulations of clinical dose distributions," Phys Med Biol 45, 459-478 (2000).

${ }^{23}$ D.R. White, H.Q. Woodard, and S.M. Hammond, "Average soft-tissue and bone models for use in radiation dosimetry," Br J Radiol 60, 907-913 (1987).

${ }^{24}$ H.Q. Woodard and D.R. White, "The composition of body tissues," Br J Radiol 59, 1209-1218 (1986). 


\section{CHAPTER}

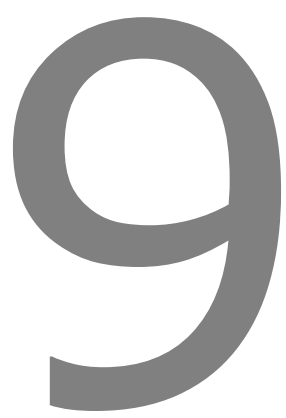

Deriving concentrations of oxygen and carbon in human tissues using single and dual energy CT for ion therapy applications

Guillaume Landry, Katia Parodi, Joachim E. Wildberger, Frank Verhaegen

Published in: Physics in Medicine and Biology 58(15):5029-5048, 2013. 


\section{ABSTRACT}

Introduction Dedicated methods of in-vivo verification of ion treatment based on the detection of secondary emitted radiation, such as Positron-Emission-Tomography (PET) and prompt gamma detection require high accuracy in the assignment of the elemental composition. This especially concerns the content in carbon and oxygen, which are the most abundant elements of human tissue. The standard single energy CT (SECT) approach to carbon and oxygen concentration determination has been shown to introduce significant discrepancies in the carbon and oxygen content of tissues. We propose a dual energy CT (DECT) based approach for carbon and oxygen content assignment and investigate the accuracy gains of the method.

Materials and methods SECT and DECT Hounsfield units (HU) were calculated using the stoichiometric calibration procedure for a comprehensive set of human tissues. Fit parameters for the stoichiometric calibration were obtained from phantom scans. Gaussian distributions with standard deviations equal to those derived from phantom scans were subsequently generated for each tissue for several values of the computed tomography dose index (CTDI ${ }_{\text {vol }}$ ). The assignment of \%weight carbon and oxygen (\%wC, $\%$ wO) was performed based on SECT and DECT. The SECT scheme employed a HU vs $\% w C, O$ approach while for DECT we explored a $Z_{\text {eff }}$ vs \%wC,O approach and a $\left(Z_{\text {eff, }}, \rho_{\mathrm{e}}\right)$ space approach. The accuracy of each scheme was estimated by calculating the root mean square (RMS) error on \%wC,O derived from the input gaussian distribution of $\mathrm{HU}$ for each tissue and also for the noiseless case as a limiting case. The $\left(Z_{\text {eff, }} \rho_{\mathrm{e}}\right)$ space approach was also compared to SECT by comparing RMS error for hydrogen and nitrogen (\%wH, \%wN). Systematic shifts were applied to the tissue HU distributions to assess the robustness of the method against systematic uncertainties in the stoichiometric calibration procedure.

Results In the absence of noise the $\left(Z_{\text {eff, }}, \rho_{\mathrm{e}}\right)$ space approach showed more accurate $\% w C, O$ assignment (largest error of $2 \%$ ) than the $Z_{\text {eff }}$ vs \%wC,O and $\mathrm{HU}$ vs \%wC,O approaches (largest errors of $15 \%$ and $30 \%$ respectively). When noise was present, the accuracy of the $\left(Z_{\mathrm{eff}}, \rho_{\mathrm{e}}\right)$ space (DECT approach) was decreased but the RMS error over all tissues was lower than the HU vs \%wC,O (SECT approach) $(5.8 \% w C$ vs. $7.5 \% w C$ at $\mathrm{CTDI}_{\mathrm{vol}}=20 \mathrm{mGy}$ ). The DECT approach showed decreasing RMS error with decreasing image noise (or increasing $C T D I_{\text {vol }}$ ). At $C T_{D I} I_{\text {vol }}=80 \mathrm{mGy}$ the RMS error over all tissues was $3.7 \%$ for DECT and $6.2 \%$ for SECT approaches. However, systematic shifts greater than $\pm 5 \mathrm{HU}$ undermined the accuracy gains afforded by DECT at any dose level.

Conclusion DECT provides more accurate \%wC,O assignment than SECT when imaging noise and systematic uncertainties in $\mathrm{HU}$ values are not considered. The presence of imaging noise degrades the $\mathrm{DECT}$ accuracy on \%wC,O assignment but it remains superior to SECT. However, DECT was found to be sensitive to systematic shifts of human tissue $\mathrm{HU}$. 


\section{INTRODUCTION}

Since more than a decade, single energy computed tomography (SECT) is routinely used to provide the basic anatomical information for realistic three-dimensional modelling of the patient geometry in clinical radiotherapy practice. In particular, patient-specific CT data are used by analytical treatment planning systems to deduce the electron density which is required for correction of tissue inhomogeneities in dose calculation of photon therapy. Moreover, they provide the basis for a semi-empirical calibration of the water-equivalent pathlength in order to account for the longitudinal influence of tissue heterogeneities in ion beam therapy treatment planning ${ }^{1,2}$.

In specialized applications such as Monte Carlo calculations, additional information on the elemental tissue composition and mass density is required. This can also be extracted from stoichiometric calibration of the patient CT data, as addressed by several studies ${ }^{3,4}$ and reviewed in this chapter. For dose calculations in high-energy photon therapy, a coarse segmentation in four different tissue types (air, lung, soft tissue and bone) and a piecewise linear interpolation of the mass density is typically sufficient, as utilized in well established Monte Carlo codes such as BEAMnrc ${ }^{5}$. However, misassignment of media may still result in significant dose errors ${ }^{6}$.

For ion beams, a finer subdivision of at least 13 different tissue types but continuous density variation is required for accurate Monte Carlo dosimetric computations in proton therapy ${ }^{7}$. However, for dedicated methods of in-vivo verification of ion treatment based on the detection of secondary emitted radiation, such as PositronEmission-Tomography ${ }^{8,9}$ and prompt gamma imaging ${ }^{10,11}$, the accuracy in the assignment of the elemental composition becomes more crucial. This especially concerns the content in carbon and oxygen, which are the most abundant elements of human tissue. In fact, the cross section data for positron-emission and prompt gamma production in ion interactions with carbon and oxygen nuclei exhibit a non-negligible difference both in terms of magnitude and energy dependence ${ }^{12,13}$. Moreover, considerable differences in the half-lives of the resulting $\beta$ + radionuclides (from 2 min for ${ }^{15} \mathrm{O}$ up to 20 min for ${ }^{11} \mathrm{C}$ ) and in the prominent emission lines of the characteristic prompt gamma (4.44 MeV for carbon and 6.13 MeV, 6.92 MeV and 7.12 MeV for oxygen) result in a quite different activation pattern or prompt gamma signal depending on the elemental composition of the traversed tissue. It should be mentioned that in PET monitoring the correct determination of the tissue composition is more critical for lighter ion beams like protons, which are only capable of producing positron-emitting target fragments.

In the Monte Carlo investigations reported so far, the assessment of tissue elemental composition from CT images alone has been mostly based on the method of Schneider et al. ${ }^{3}$ Despite the promising results, major shortcomings were reported in several PET studies due to the non-unique correlativity of $\mathrm{HU}$ and tissue parameters especially for abdomino-pelvic anatomical locations ${ }^{14}$ and the missing separation of white and grey matter as well as cerebrospinal fluid with considerably different carbon content in the head ${ }^{9}$. For the latter problem, more recent approaches proposed the 
usage of additional magnetic resonance imaging for improved tissue classification ${ }^{15}$ but this is, however, limited to the brain region.

Therefore, in this work we investigate the impact of the recent availability of commercial dual-energy CT (DECT) scanners ${ }^{16}$, which has enabled routine extraction of additional information on the effective atomic number $Z_{\text {eff }}$ besides electron density $\rho_{\mathrm{e}}$. So far, the impact of DECT in radiation therapy has been explored in terms of improved dosimetric calculation for external photon beam radiotherapy ${ }^{17}$ and brachytherapy ${ }^{18}$, as well as improved CT-based ion range calibration in ion beam therapy ${ }^{19}$. Therefore, this work investigates the possibility of an improved identification of the carbon and oxygen constituents, together with calcium and phosphor which are abundant in bony structures. An improved tissue classification is expected to increase the accuracy in the prediction of radiation-induced positron emitters and prompt gamma production, thus reducing one of the largest sources of uncertainty for in-vivo range verification in clinical ion beam therapy ${ }^{20}$.

\section{PROPERTIES OF HUMAN TISSUES}

For this study we considered 73 human tissues whose density and elemental composition were obtained from Woodard and White ${ }^{21,22}$. This list is the same as in Schneider et $a l^{3}{ }^{3}$ with the addition of two bladder tissues, filled and empty, and is the most recent data used in radiotherapy. In this section we briefly illustrate how the elemental components of tissues correlate with $\mathrm{HU}, Z_{\text {eff }}$ and $\rho_{\mathrm{e}}$. Figure 9.1 shows how the carbon, oxygen, phosphorous and calcium concentrations of each tissue are distributed as a function of $\mathrm{HU}$ (data from Schneider et al. ${ }^{3}$ ). As mentioned in this reference, there is considerable spread of the data and no simple one-to-one relationship with $\mathrm{HU}$ can be established. Plotting the same data as a function of $Z_{\text {eff }}$ :

$$
Z_{\text {eff }}=\sqrt[n]{\sum_{i} \frac{Z_{i}}{A_{i}} \omega_{i} Z_{i}^{n} / \sum_{i} \frac{Z_{i}}{A_{i}} \omega_{i}}
$$

calculated using the mass fraction $\omega_{i}$ of element $i$ having atomic number $Z_{i}$ and atomic mass $A_{i}^{23}$ ), with $n=3.3$, shows that for tissues with $Z_{\text {eff }}$ between 6 and 8 there appears to be a better correlation of the data (Figure 9.2), although there is still a significant spread of the data. We also observe a better correlation between the concentration of phosphorous and calcium with $Z_{\text {eff }}$ than with $\mathrm{HU}$, which will be discussed later. If one wishes to employ all data obtained from DECT scanning, then it is necessary to take $\rho_{\mathrm{e}}$ into account. Figure 9.3 shows a scatter plot of all tissues in a $\left(Z_{\text {eff }}, \rho_{\mathrm{e}}\right)$ space. Tissues have been labelled as belonging either to the head, thorax or abdomen regions. $A$ tissue can belong to several regions. While there is a general correlation between $Z_{\text {eff }}$ and $\rho_{\mathrm{e}}$ for human tissues, we see that for soft tissues there is added separation by employing the two quantities to identify tissues. In this study we attempted to im- 
prove the assignment of carbon and oxygen concentrations by developing schemes based on $Z_{\text {eff }}$ and $\rho_{\mathrm{e}}$ and the data presented in Figures 9.2 and 9.3. These schemes are presented in the next section.
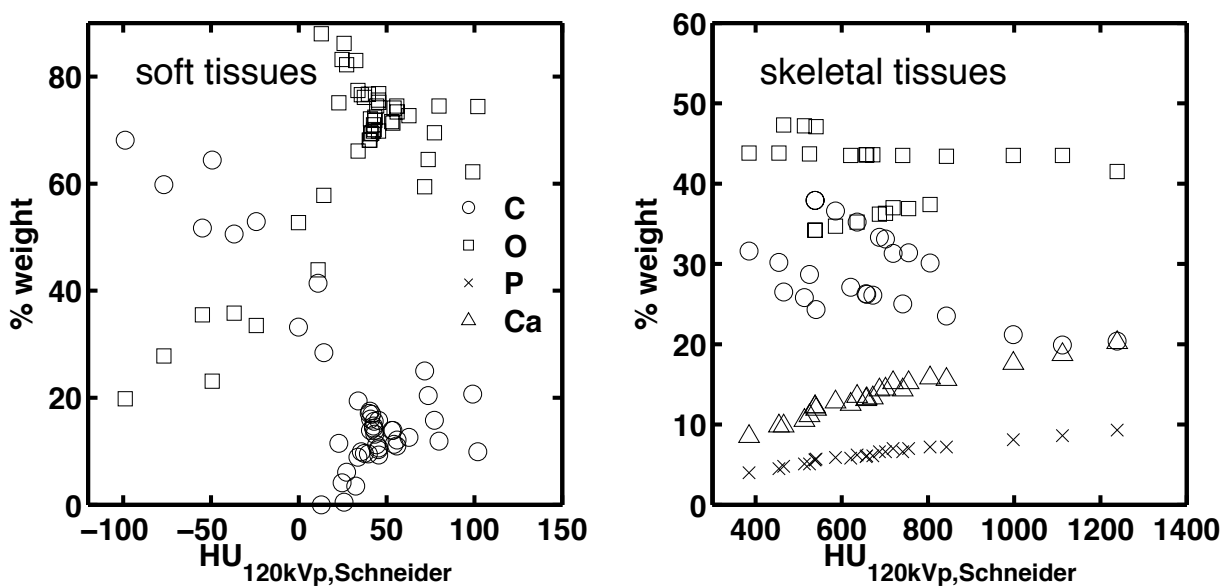

Figure 9.1: Left The \% weight of elements $C$ and $O$ for human soft tissues vs. the HU at $120 \mathrm{kVp}$ calculated using the formalism and fit parameters of Schneider et al. Right The \% weight of elements C, O, P and Ca for human skeletal tissues vs. the HU at $120 \mathrm{kVp}$ calculated as in Left.
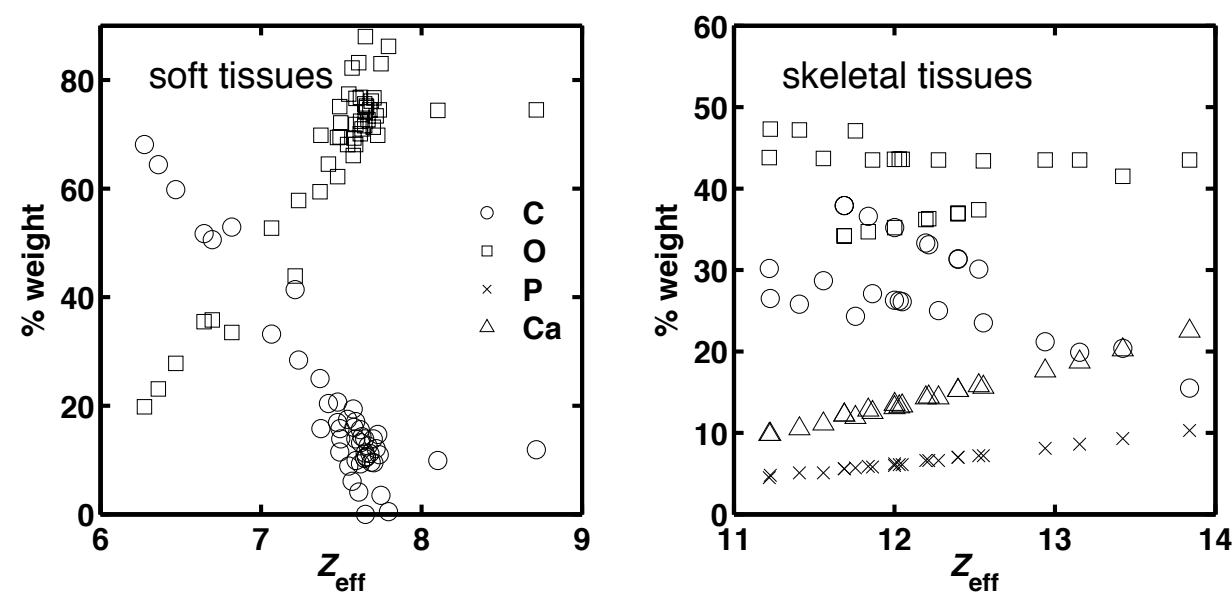

Figure 9.2: Left The \% weight of elements $C$ and $O$ for human soft tissues vs. the effective atomic number $Z_{\text {eff }}$ calculated from the tabulated compositions. Right The \% weight of elements $C, O, P$ and $C a$ for human skeletal tissues vs. $Z_{\text {eff. }}$. 

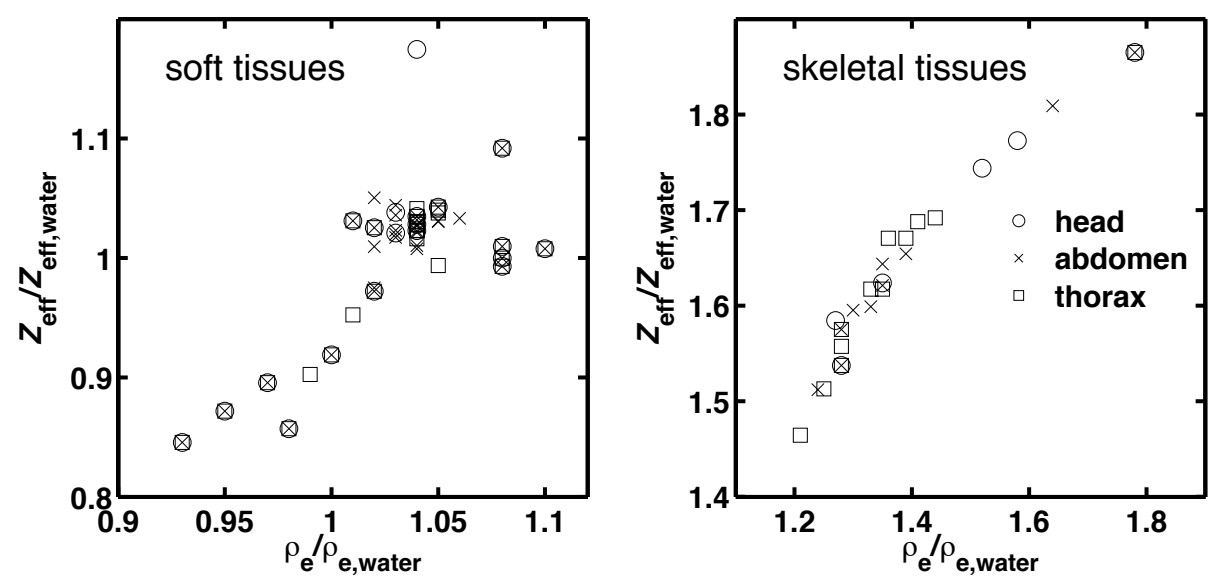

Figure 9.3: Left $Z_{\text {eff }} / Z_{\text {eff,water }} v s . \rho_{e} / \rho_{e, \text { water }}$ scatter plot for soft tissues and Right skeletal tissues showing the potential separation of human tissues afforded by DECT scanning.

\section{MATERIALS AND METHODS}

In this section we present the assignment schemes we developed for the concentrations of carbon and oxygen based on DECT scanning. We also present the SECT approach of Schneider et al. for comparison. For all schemes, it was necessary to explore the influence of image noise and generate $\mathrm{HU}$ for reference human tissues. Parameters were thus derived from phantom measurements. These experiments are described below.

\section{Estimating noise levels in SECT and DECT scanning}

A CIRS (Norfolk, VA) model 002H5 IMRT phantom made of water equivalent plastic representing average patient body size was scanned at a dual source DECT scanner (SOMATOM Definition FLASH, Siemens Healthcare, Forchheim, Germany). Scans were taken at different imaging dose levels (reported using the computed tomography dose index $\mathrm{CTDI}_{\text {vol }}$ ) with a single energy of $120 \mathrm{kVp}$ and in dual energy mode with the low energy beam set to $80 \mathrm{kVp}$ and the high energy beam set to $140 \mathrm{kVp}$ with a Tin filter. The SECT and DECT scans had the same CTDI ${ }_{\text {vol }}=10 \mathrm{mGy}, 20 \mathrm{mGy}$ and $40 \mathrm{mGy}$. Tube current modulation (CareDose) was disabled. Images were reconstructed with $3 \mathrm{~mm}$ slice thickness using either filtered backprojection (FBP) (B30f filter for SECT, D30f for $D E C T$ ) or by using Siemens' iterative reconstruction (IR) algorithm (SAFIRE, I30f $\backslash 5$ for SECT, Q30f $\backslash 5$ for DECT). Noise was estimated by measuring the standard deviation of $\mathrm{HU}$ for each $\mathrm{kVp}$ and $\mathrm{CTDI}_{\mathrm{vol}}$ in a circular region of interest at the center of the phantom $\left(2.2 \mathrm{~cm}^{2}\right)$. 


\section{Generating a human tissue dataset}

\section{Stoichiometric parameters}

For SECT, we employed the stoichiometric fit parameters from Schneider et $a l^{3}$ to calculate $\mathrm{HU}_{120 \mathrm{kV} \text {, Schneider }}$ for the human tissues considered in this study. We used these parameters instead of our own to enable us to make direct use of the segmentation procedure of Schneider et al. (further explained below). To calculate $\mathrm{HU}_{80 \mathrm{kVp}}$ and $\mathrm{HU}_{140 \mathrm{kVp}}$ for DECT it was necessary to obtain the fit parameters $k_{1,140 \mathrm{kV},}, k_{2,140 \mathrm{kV},}, k_{1,80 \mathrm{kVp}}$, $k_{2,80 \mathrm{kV} \text {. }}$. This was achieved by scanning a RMI 465 phantom (Gammex Inc., Middleton, WI) containing several tissue mimicking inserts with the Siemens DECT scanner ${ }^{24}$ and performing the stoichiometric calibration. The stoichiometric calibration method is well accepted and has been used in similar work before ${ }^{19}$.

\section{HU dataset}

To obtain a noisy dataset representative of a SECT and DECT scan, we performed the following at each kVp. For each tissue, a HU Gaussian distribution of 500 simulated measurements was generated with mean $\mathrm{HU}$ equal to that calculated with the stoichiometric procedure and standard deviation equal to that derived in the noise estimation experiment described above (using IR data). It was assumed that the noise in the high and low kVp DECT images was uncorrelated as the scanner has dual x-ray sources. To explore the effect of increasing the imaging dose, we used the standard deviations obtained with $\mathrm{CTDI}_{\mathrm{vol}}=20 \mathrm{mGy}$ and scaled them according to $\sigma_{\mathrm{HU}} \propto \mathrm{CTDI}_{\mathrm{vol}}{ }^{-2}$. This relation was experimentally verified for $\mathrm{CTDI}_{\mathrm{vol}}=10 \mathrm{mGy}, 20 \mathrm{mGy}$ and $40 \mathrm{mGy}$.

For each noise level, secondary datasets were generated where the mean $\mathrm{HU}$ were shifted by $-20,-10,-5,5,10$ and $20 \mathrm{HU}$ to investigate the robustness against intrinsic uncertainties in the $\mathrm{HU}$ calibration.

\section{Assigning \%weight $\mathrm{C}$ and \%weight $\mathrm{O}$ based on SECT}

For SECT the carbon and oxygen concentrations were assigned for a given $\mathrm{HU}_{120 \mathrm{kV} p}$ following the work of Schneider et al. They proposed two approaches, one based on piecewise fits of the data presented in Figure 9.1 and one based on HU bins (Table 6 of Schneider et al. $^{3}$ ). The table approach makes use of the average elemental composition of all tissues in a bin. These two schemes are called SECT-fits and SECT-table and are shown in Figure 9.4. Other publications have generally made use of the SECT-table approach $^{14,26}$ for its simplicity of implementation. The two approaches are very similar, with main differences at the sharp peak/valley of the SECT-fits approach. The differ- 
ence between the two approaches was investigated with results presented in a later section.
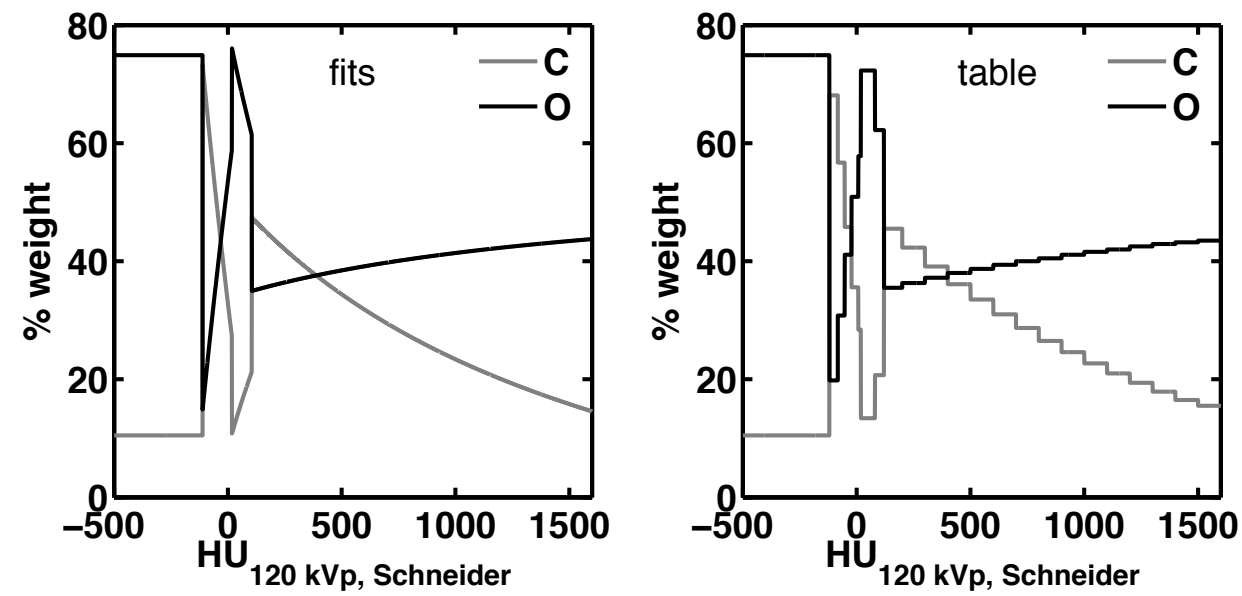

Figure 9.4: Left The \%weight assignment for carbon and oxygen vs. HU at $120 \mathrm{kVp}$ corresponding to the fits proposed in Schneider et al. Right The \%weight assignment for carbon and oxygen vs. $\mathrm{HU}$ at $120 \mathrm{kVp}$ corresponding to Table 6 in Schneider et al.

\section{Assigning \%weight $\mathrm{C}$ and \%weight $\mathrm{O}$ based on DECT}

\section{Extracting $Z_{\text {eff }}$ and $\rho_{e}$ from DECT scans}

In the following we employed the quantity $Z_{\text {eff, }}$ obtained from DECT scanning. Plotting $Z_{\text {eff }}$ vs. $\mu_{80 \mathrm{kVp}} / \mu_{140 \mathrm{kVp}}$ for human tissues (using the stoichiometric fit parameters to generate linear attenuation coefficients relative to water $\mu_{80 \mathrm{kVp}}$ and $\mu_{140 \mathrm{kVp}}$ ) shows a convenient one-to-one relationship between the two (Figure 9.5). We used this relationship to convert simulated DECT measurements to $Z_{\text {eff. }}$. 


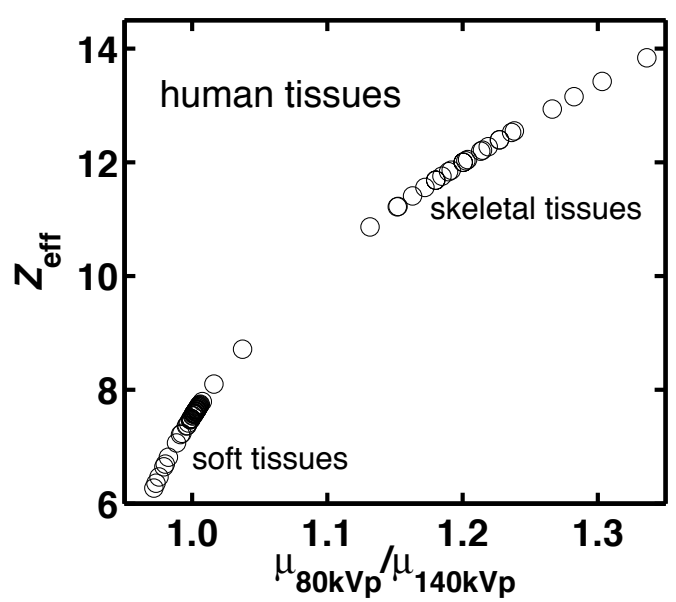

Figure 9.5: $Z_{\text {eff }}$ calculated from tabulated human tissue compositions vs. the ratio of the linear attenuation coefficient $\mu$ obtained at $80 \mathrm{kVp}$ and $140 \mathrm{kVp}$. The HU for human tissues have been calculated using the stoichiometric calibration procedure using data from a Gammex RMI phantom scanned at a dual source $D E C T$ scanner. The excellent one-to-one relation of $Z_{\text {eff }}$ and $\mu_{80 \mathrm{kv}} / \mu_{140 \mathrm{kvp}}$ for human tissues permits conversion between these two quantities.

We derived $\rho_{\mathrm{e}}$ from the simulated measurements using the dual energy subtraction method of Saito ${ }^{25}$, using the fit parameters (Table 3 in Saito, 2012) which were derived from the data of Landry et $a .^{24}$. These data were obtained with the same scanner and Gammex RMI 465 phantom used in this study and used to obtain the stoichiometric fit parameters. The dual energy subtraction method exploits the excellent linearity between $\rho_{\mathrm{e}}$ and a weighted subtraction of the high and low $\mathrm{kVp} H \mathrm{HU}$.

\%weight $\mathrm{C}, \mathrm{O}$ vs. $Z_{\text {eff }}$

Our first DECT assignment scheme used fits to the data of Figure 9.2 for $Z_{\text {eff }}$ between 6 and 8 . Outside this range there appears to be little added benefit to using $Z_{\text {eff }}$ instead of $\mathrm{HU}$ since the two are well correlated for skeletal tissues. While intuitive, this approach does not make full use of all the information afforded by DECT scanning as it neglects $\rho_{\mathrm{e}}$. 


\section{$\left(Z_{\text {eff }}, \rho_{e}\right)$ space approach}

In this approach we use the composition of human tissues to generate a tissue $\left(Z_{\text {eff }}, \rho_{\mathrm{e}}\right)$ space (Figure 9.3). In this space it is possible to minimize the distance between a measurement $\left[Z_{\text {eff, }}, \rho_{\mathrm{e}}\right]$ and each reference tissue (we refer to the tissue space as $\left(Z_{\text {eff }}\right.$ ,$\rho_{\mathrm{e}}$ ) and measurements as $\left.\left[Z_{\mathrm{eff}}, \rho_{\mathrm{e}}\right]\right)$. We assign the tissue with the least $2 \mathrm{D}$ distance to the measurement. As there is a degree of correlation between $Z_{\text {eff }}$ and $\rho_{\mathrm{e}}$ (see Figure 12 in Landry et $a .^{18}$ ) we make use of the Mahalanobis distance instead of the Euclidian distance. To achieve this we need an estimate of the covariance matrix in the $\left(Z_{\text {eff }}, \rho_{\mathrm{e}}\right)$ space for each tissue type. We derived it using our simulated HU dataset. When minimizing the distance between measurements and reference tissues in the map, we restricted the tissue list to those relevant to one of three anatomical regions; the head, abdomen and thorax.

\section{Assessing accuracy of \%weight carbon and oxygen assignment for SECT and DECT}

The accuracy of SECT and DECT assignment schemes was assessed by the root mean square (RMS) error on \%weight C (\%wC) and \%weight O (\%wO) over the 500 simulated measurements of each tissue. Additionally the maximum RMS error and total RMS error over the $73 \times 500$ simulated measurements were reported for each dose level and assignment scheme. As hydrogen and nitrogen are major constituents of human tissues, their maximum RMS error and total RMS error were also reported using SECT and the DECT $\left(Z_{\text {eff }}, \rho_{\mathrm{e}}\right)$ space approach. The RMS errors are always reported as \%weight.

The robustness to systematic shift of $\mathrm{HU}$ which may result from the stoichiometric calibration procedure was evaluated by performing the same procedure for the shifted HU distributions.

\section{RESULTS AND DISCUSSION}

\section{Noise levels}

The noise levels from SECT and DECT scans are reported in Table 9.1. The noise levels of the $120 \mathrm{kVp}$ scan (SECT) are lower than those of the $80 \mathrm{kVp}$ and $140 \mathrm{kVp}$ scans (DECT) because DECT scanning is performed at equivalent dose as SECT scanning. Each image from DECT scanning is thus acquired at roughly half the dose. Noise levels from IR were roughly half of those from FBP. 
Table 9.1: Noise levels from SECT and DECT scans with FPB and IR image reconstruction. For both SECT and DECT scans CTDI $\mathrm{vol}=20 \mathrm{mGy}$.

\begin{tabular}{|c|c|c|c|}
\hline Image reconstruction & $\sigma_{120 \mathrm{kV}, \text { SECT }}(\mathrm{HU})$ & $\sigma_{80 \mathrm{k} \mathrm{V}_{\mathrm{D}, \mathrm{DECT}}}(\mathrm{HU})$ & $\sigma_{140 \mathrm{kV}, \mathrm{DECT}}(\mathrm{HU})$ \\
\hline $\mathrm{FBP}$ & 8.4 & 14 & 13 \\
\hline IR & 4.4 & 6.5 & 6.1 \\
\hline
\end{tabular}

\section{Stoichiometric fit parameters}

The stoichiometric fit parameters derived from our DECT scans are reported in Table 9.2. The largest difference between the HU predicted by the stoichiometric procedure and the ones we measured for the Gammex RMI 465 phantom were $55 \mathrm{HU}$ at $80 \mathrm{kVp}$ $\left(\mathrm{CB} 2-50 \% \mathrm{CaCO}_{3}\right)$ and $30 \mathrm{HU}$ at $140 \mathrm{kVp}\left(\mathrm{CB} 2-30 \% \mathrm{CaCO}_{3}\right)$. The average absolute differences over all inserts were $24 \mathrm{HU}$ and $16 \mathrm{HU}$ respectively.

Table 9.2: Stoichiometric fit parameters from scanning the Gammex RMI 465 phantom at a DECT scanner at $80 \mathrm{kVp}$ and $140 \mathrm{kVp}$ with Tin filter.

\begin{tabular}{ccc}
\hline \hline$k V p$ & $k_{1}$ & $k_{2}$ \\
\hline 80 & 0.002225 & $4.315 \times 10^{-5}$ \\
140 & 0.001448 & $8.927 \times 10^{-6}$ \\
\hline \hline
\end{tabular}

\section{Accuracy of SECT and DECT approaches in the absence of noise}

SECT

Figure 9.6 plots the residuals on \% weight of carbon and oxygen from applying the SECT-table scheme of Figure 9.4 for all tissues except lung, which is correctly assigned (lung is not shown because its $\mathrm{HU}=-744$ ). We see that in the soft tissue range $[-100 \mathrm{HU}$, $100 \mathrm{HU}$ ] there are errors of up to $30 \%$ weight on the assignment of carbon and oxygen. This is expected from Figure 9.1. For skeletal tissues the errors are confined to $\pm 10 \%$. Similar results are obtained with the SECT-fits scheme. 


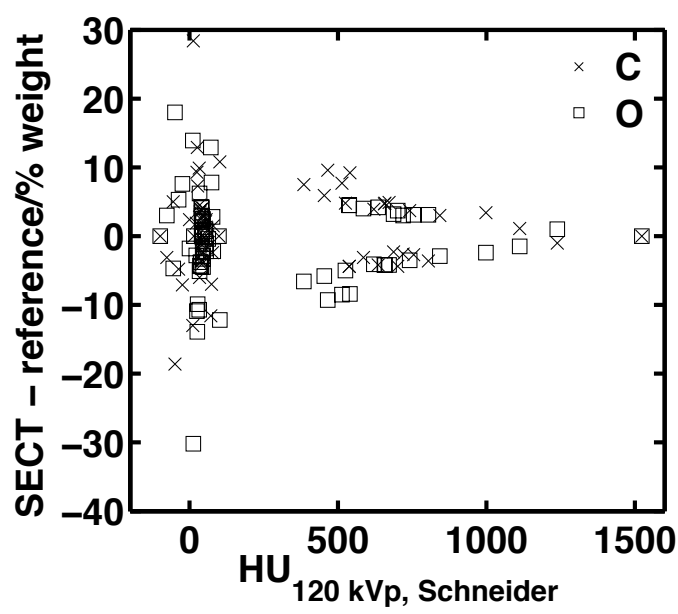

Figure 9.6: Residuals on \%weight for carbon and oxygen derived from $\mathrm{HU}$ at $120 \mathrm{kVp}$ SECT in the absence of noise using the stoichiometric calibration approach.

\section{$D E C T$}

$\%$ weight $\mathrm{C}, \mathrm{O}$ vs. $Z_{\text {eff }}$

The fitting of \%wC vs. $Z_{\text {eff }}$ yielded $\% w C=-42.27 \times Z_{\text {eff }}+334.2$ with $R^{2}=0.9326$. For oxygen we obtained $\% w O=-41.15 \times Z_{\text {eff }}-239.9$ with $R^{2}=0.9087$. Figure 9.7 plots the residuals for carbon and oxygen \%weight fractions derived from $Z_{\text {eff }}$ for soft tissues between $Z_{\text {eff }}$ 6 and 8. The maximum residuals are lower than those for the SECT-table approach shown in Figure 9.6 (15\% instead of 30\%). Comparing Figures 9.6 and 9.7 does not show a clear advantage of representing the data vs. $Z_{\text {eff }}$ instead of HU. Additionally, at the same imaging dose, $Z_{\text {eff }}$ is inherently noisier than $\mathrm{HU}$ as it is derived from a ratio of the high and low linear attenuation coefficient measurements (Figure 9.5). 


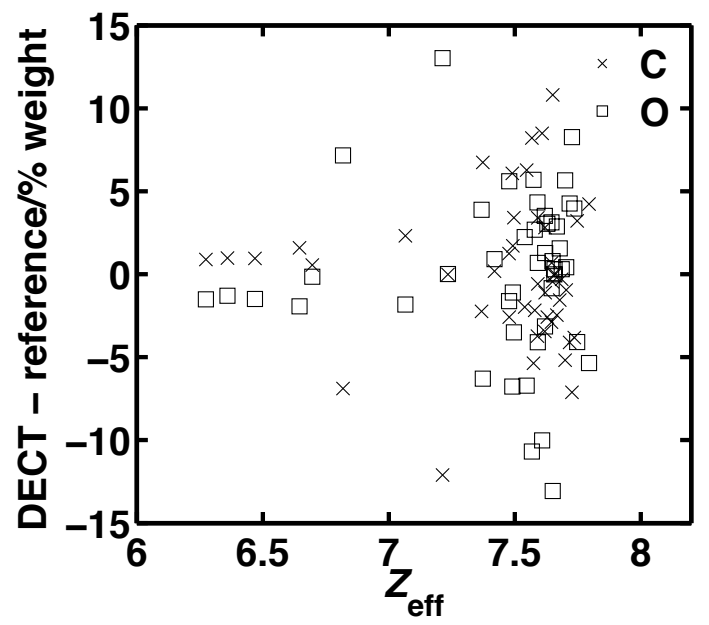

Figure 9.7: Residuals on \%weight for carbon and oxygen derived from $Z_{\text {eff }}$ from $D E C T$ in the absence of noise. The data is restricted to soft tissues with $Z_{\text {eff }}$ between 6 and 8 .

$\%$ weight $P, C a$ vs. $Z_{\text {eff }}$ and $H U_{120 k v p}$

A similar exercise as above was performed for the concentrations of phosphorous and calcium. These were fitted against $\mathrm{HU}_{120 \mathrm{kVp}}(\mathrm{SECT})$ and $Z_{\text {eff }}(\mathrm{DECT})$. In this case we did not use SECT-fits or SECT-table but a rational function with a first order polynomial as numerator and denominator. For DECT we used a power function of the form $a x^{b}+c$. For both phosphorous and calcium the maximum residual and the RMS error on all skeletal tissues was reduced by a factor 2 when using DECT (0.18 RMS error \%weight $P$, 0.09 RMS error \%weight $\mathrm{Ca}$ ) instead of SECT (0.43 RMS error \%weight $\mathrm{P}, 0.2 \mathrm{RMS}$ error \%weight (Ca). These findings are similar to those reported by Yang et al. ${ }^{27}$ Whether this is a relevant improvement needs evaluation which is outside the scope of this study. 


\section{$\left(Z_{\text {eff }}, \rho_{e}\right)$ space approach}

In the absence of noise it is possible to assign the carbon and oxygen concentration within an accuracy of $2 \%$ weight fraction when using the $\left(Z_{\text {eff }}, \rho_{\mathrm{e}}\right)$ space, indicating that there is little overlap of the average $\left[Z_{\text {eff, }}, \rho_{\mathrm{e}}\right]$ values for human tissues. This is the limiting case of the DECT approach based on the properties of the reference human tissues.

\section{Assessing accuracy in the presence of realistic imaging noise levels}

The presence of image noise greatly impacts the results of the previous sections. The three following sections present the influence of simulated image noise on the accuracy of SECT and DECT based \%weight assignment for carbon and oxygen.

\section{Comparing SECT-fits and SECT-table}

Figure 9.8 presents the RMS error for each tissue at the $C T D I_{\text {vol }}=20 \mathrm{mGy}$ dose level for the two SECT based schemes for \%weight carbon and oxygen. The tissues are ordered by mass density. We see that the two schemes yield similar results across the tissues investigated, as can be expected when comparing them in Figure 9.4. Several tissues have RMS error $>10 \%$ weight, as expected from Figure 9.6. Red bone marrow, yellow/red bone marrow yellow bone marrow, brain (or cerebrospinal) fluid, urine and cartilage yielded the largest errors. The largest error (30\%weight) was for brain fluid which is similar to water and contains no carbon. The schemes of Figure 9.4 always yield \%wC $>15 \%$ for any $\mathrm{HU}$, thus any water-like substance within the body will suffer from misassignment. As SECT-fits and SECT-table are similar, we present results for SECT-table in the following section. 

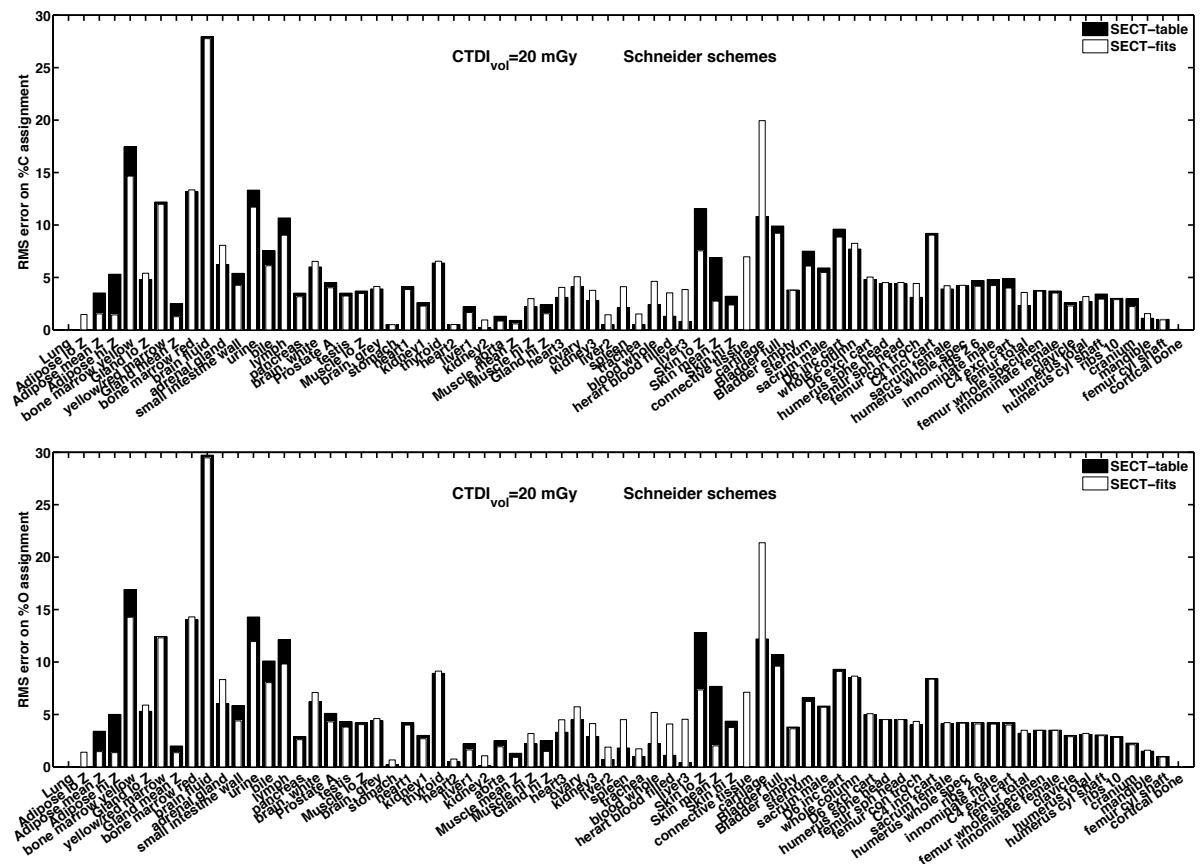

Figure 9.8: Top RMS error on \%weight C for SECT-fits and SECT-table for CTDI ${ }_{\text {vol }}=20 \mathrm{mGy}$. Bottom Same for \%weight $O$.

Comparing SECT-table and DECT- $\left(Z_{\text {eff }}, \rho_{e}\right)$

The RMS errors for the SECT-table and DECT approaches are compared in Figures 9.99.12 for two imaging dose levels (CTDI ${ }_{\text {vol }}=20 \mathrm{mGy}$ and $80 \mathrm{mGy}$ ). For DECT only results of the $\left(Z_{\text {eff }}, \rho_{\mathrm{e}}\right)$ map approach are reported as the \%C,O vs. $Z_{\text {eff }}$ approach yield only a slight reduction of residual errors when compared to SECT in the noiseless scenario (Figure 9.7). Here the results are separated into the three anatomical regions (head, thorax, abdomen).

At $20 \mathrm{mGy}$ we see some improvement from DECT for some tissues (brain fluid, yellow/red marrow, yellow bone marrow, skin lo $Z$ as well as high density bones). The presence of imaging noise greatly reduces the performance obtained in the noiseless case. For red bone marrow the RMS error is higher for DECT than SECT. When decreasing the noise to the level achieved with a CTDI ${ }_{\text {vol }}$ of $80 \mathrm{mGy}$ we see that DECT performs better than SECT for all tissues except adrenal gland and small intestine wall. At that noise level RMS errors for DECT are $<10 \%$ weight for most tissues. 

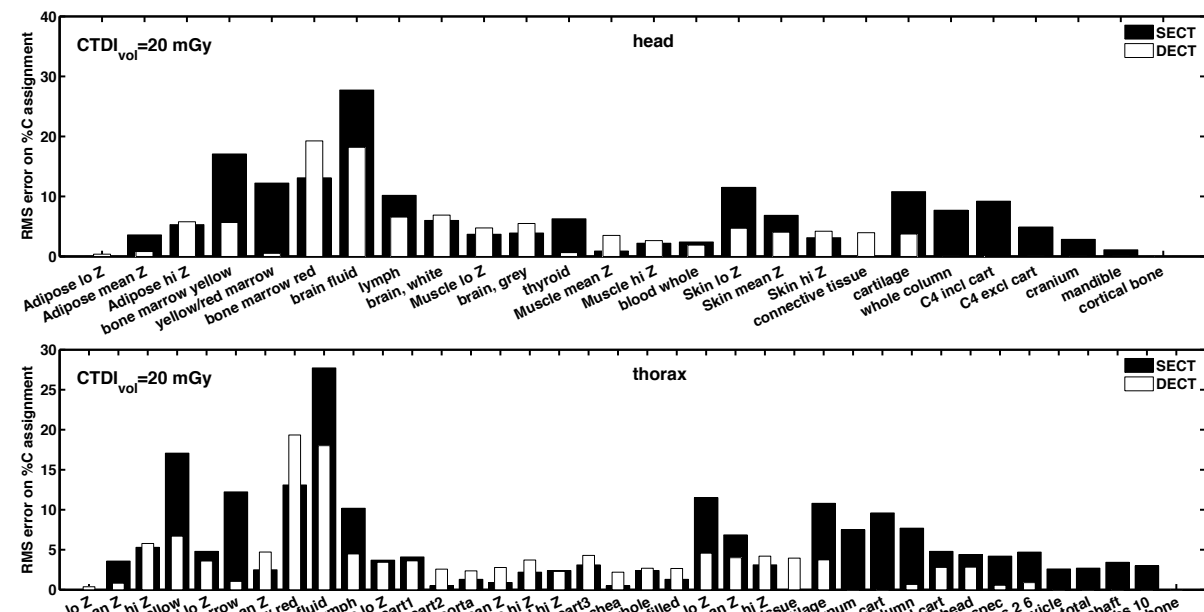

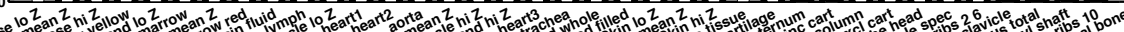

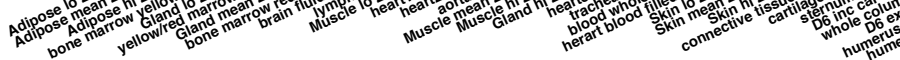

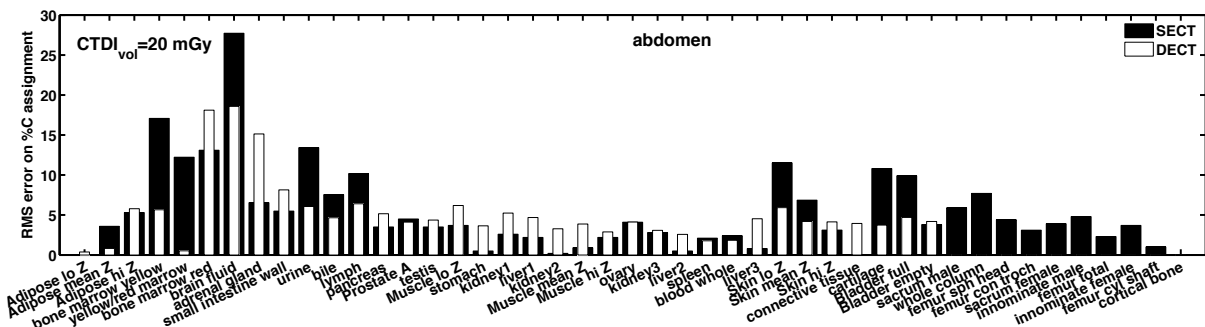

Figure 9.9: RMS error on \%C for a SECT and DECT scan (( $\left.Z_{\text {eff }}, \rho_{e}\right)$ approach) with CTDI vol $=20 \mathrm{mGy}$ separated into the head, thorax and abdomen regions. 


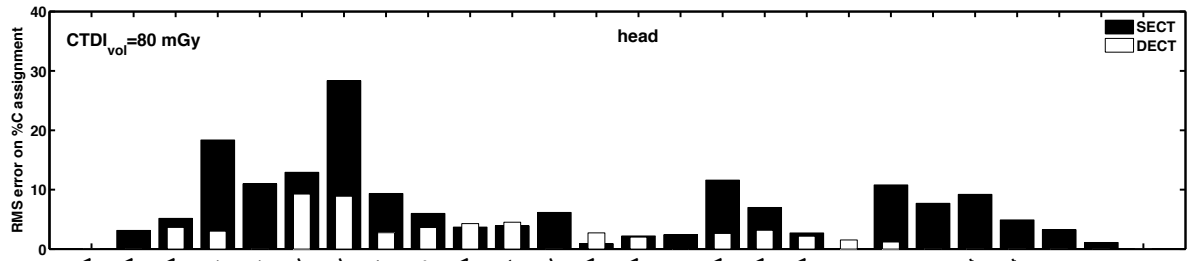

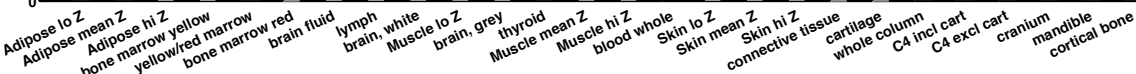

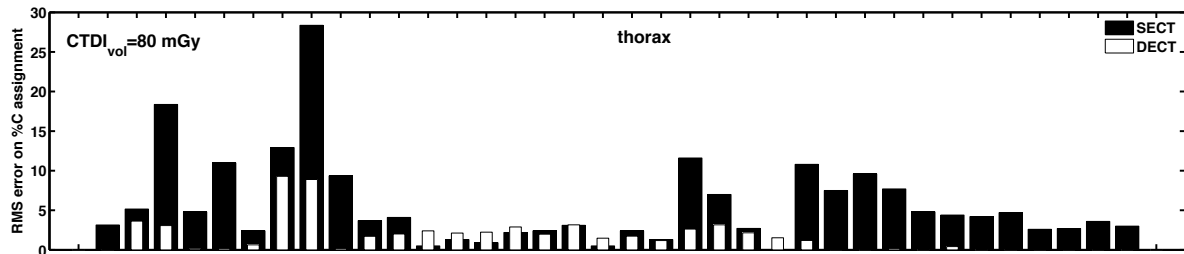

$2{ }^{2} 2$ w 2 w 2 did 2
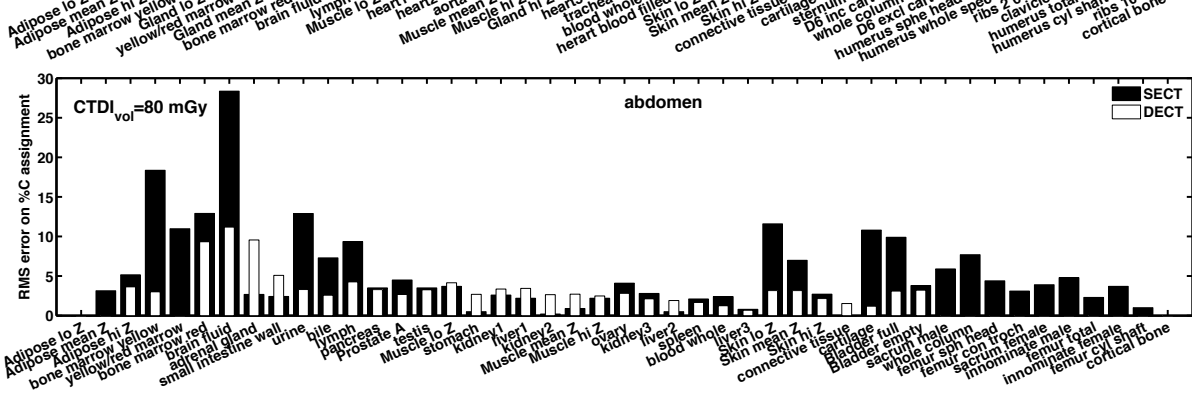

Figure 9.10: RMS error on \%C for a SECT and DECT scan $\left(\left(Z_{\text {eff }}, \rho_{e}\right)\right.$ approach) with $C T D I_{\text {vol }}=80 \mathrm{mGy}$ separated into the head, thorax and abdomen regions. 

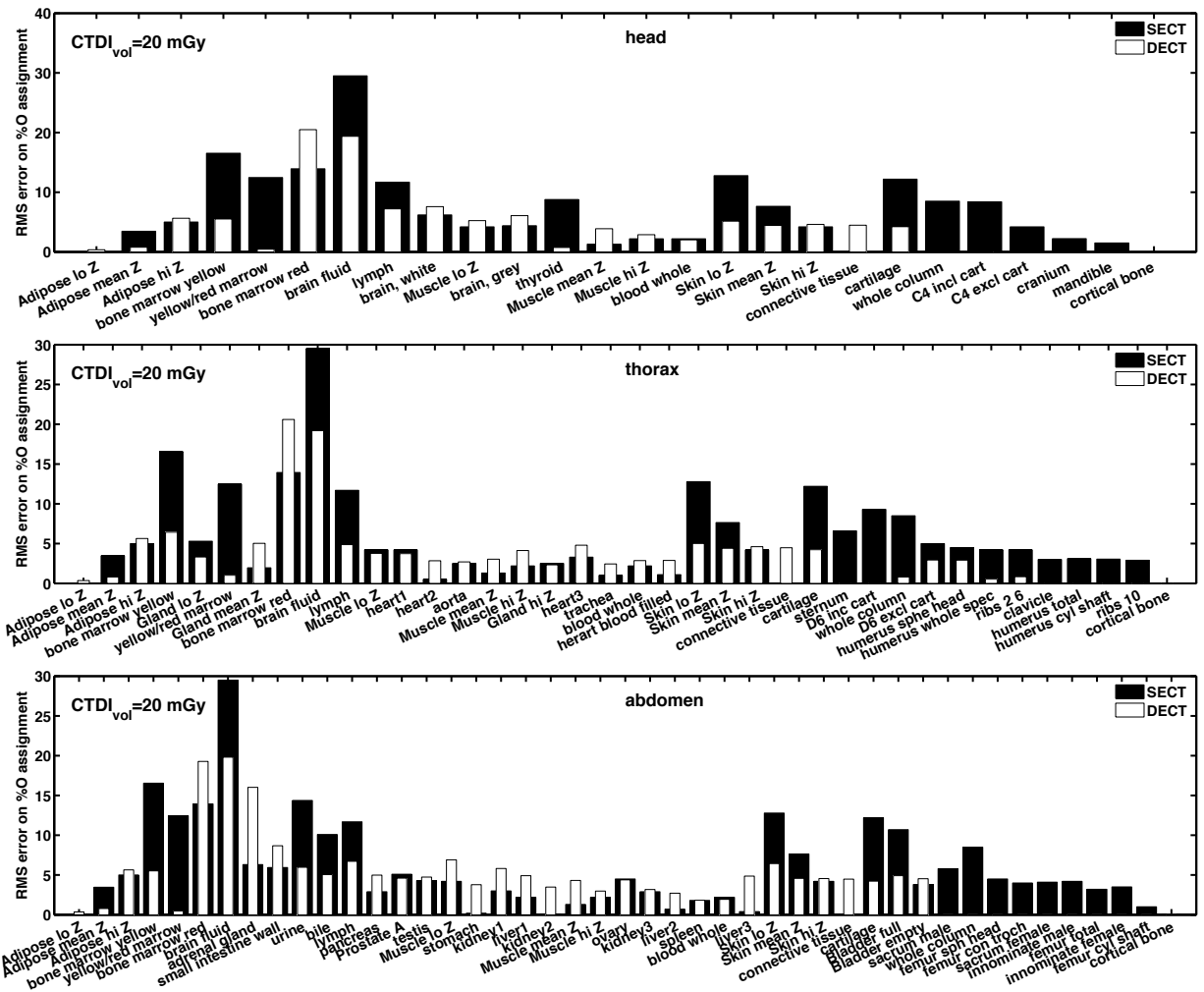

Figure 9.11: $R M S$ error on \%O for a SECT and DECT scan $\left(\left(Z_{\text {eff }}, \rho_{e}\right)\right.$ approach) with $C T D I_{\text {vol }}=20 \mathrm{mGy}$ separated into the head, thorax and abdomen regions. 

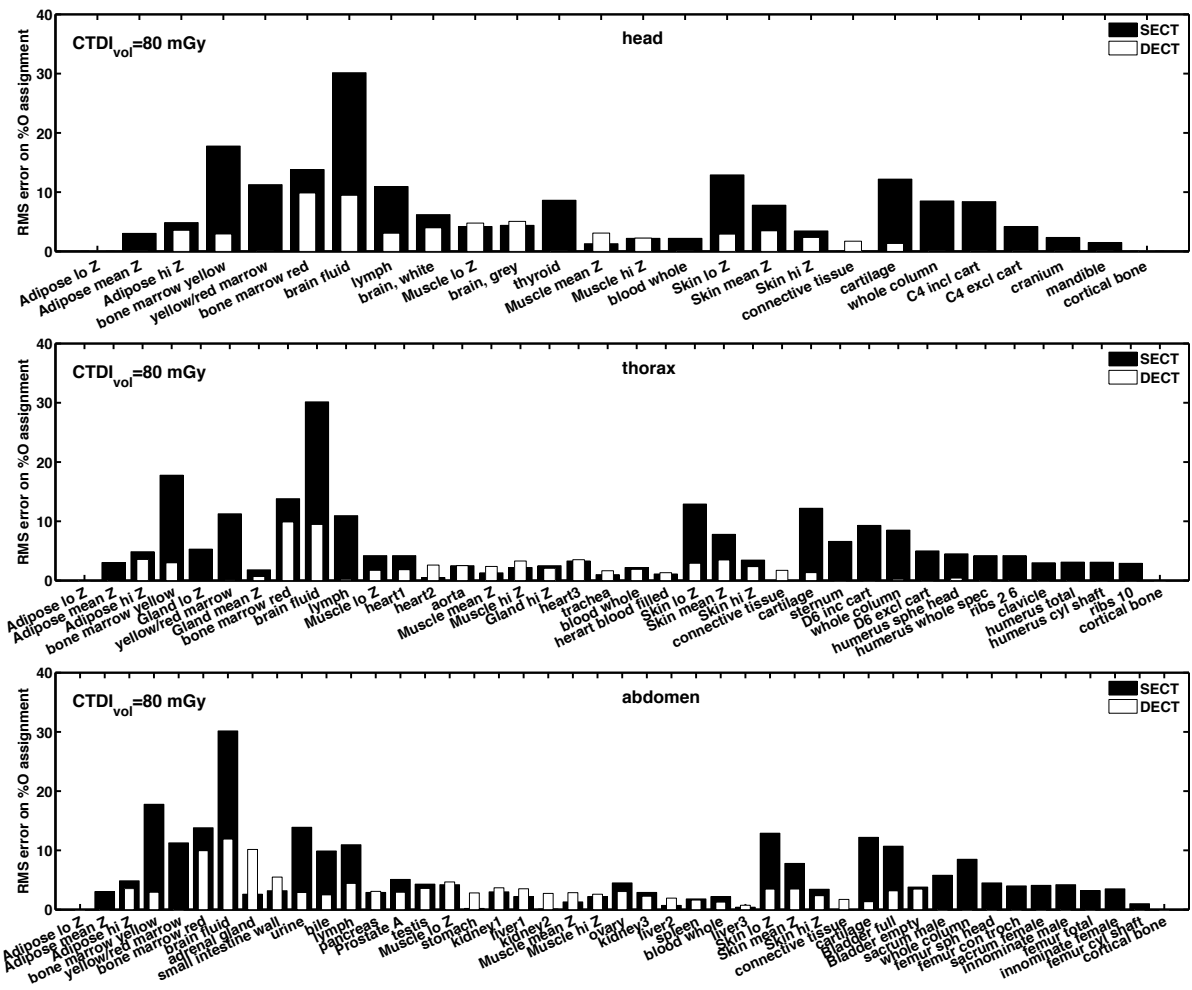

Figure 9.12: $R M S$ error on \%O for a SECT and DECT scan $\left(\left(Z_{e f f}, \rho_{e}\right)\right.$ approach) with $C T D I_{\text {vol }}=80 m G y$ separated into the head, thorax and abdomen regions.

RMS error on \%weight at different dose levels

While Figures 9.9-9.12 showed data calculated with doses of $20 \mathrm{mGy}$, and $80 \mathrm{mGy}$, maximum and total RMS errors were also calculated for $40 \mathrm{mGy}, 120 \mathrm{mGy}$ and 160 mGy. Figure 9.13 shows the maximum RMS error and the total RMS error on carbon \%weight for varying CT imaging doses while Figure 9.14 shows the same data for oxygen. For Figures 9.13 and 9.14 we only considered the abdomen region when calculating RMS errors for DECT. It was verified that the head and thorax showed similar results. The figures show that at all noise levels the DECT approach yields lower maximum and total RMS errors than the SECT approach. The CT dose scaling factor of 2 corresponds to $C T D I_{\text {vol }}=40 \mathrm{mGy}$ and the tube loading corresponding to that dose level was roughly the maximum available with our current CT protocol. Thus to achieve the improvements observed with higher dose scaling factors it may be necessary to reduce image noise by other means than varying imaging dose. While the DECT approach benefits from reduced image noise, the SECT approach shows limited $(<1 \%)$ improve- 
ment on RMS error over all tissues across the imaging dose range investigated. This is due to the intrinsic limitations of the approach presented in Figure 9.6.
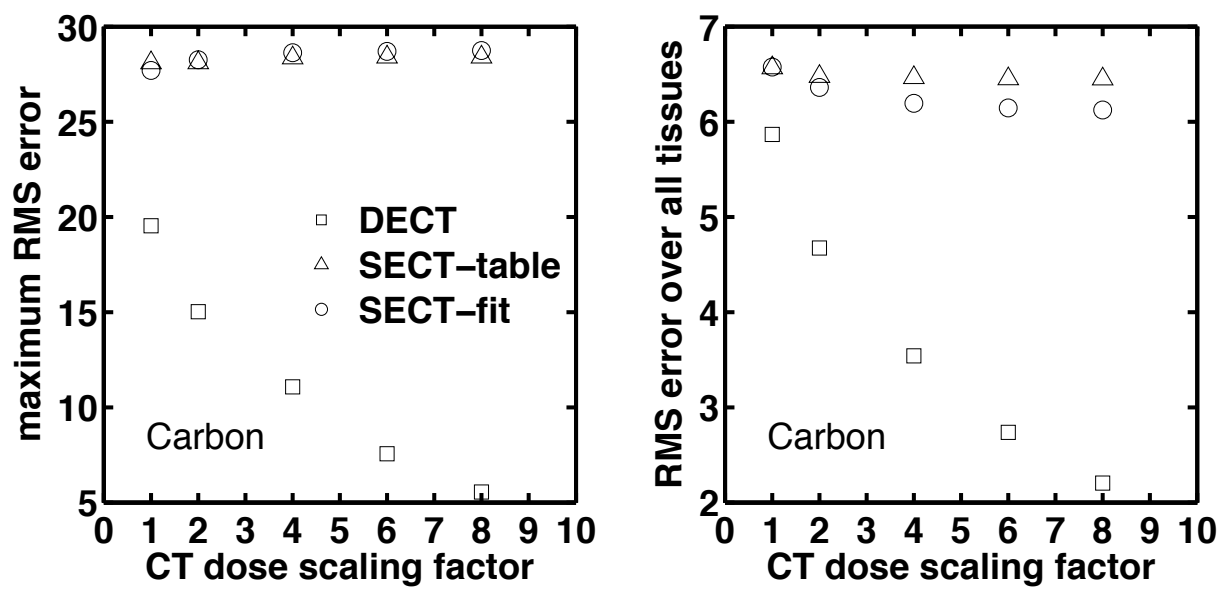

Figure 9.13: Left The maximum RMS error on \%weight $C$ assignment for DECT and SECT as a function of dose level. A CT dose scaling factor of 1 corresponds to a CTDI $\mathrm{vol}=20 \mathrm{mGy}$. Right The RMS error on \%weight $C$ for all tissues as a function of dose level. The DECT data is for the abdomen region. The behaviour of the thorax and head regions was similar.
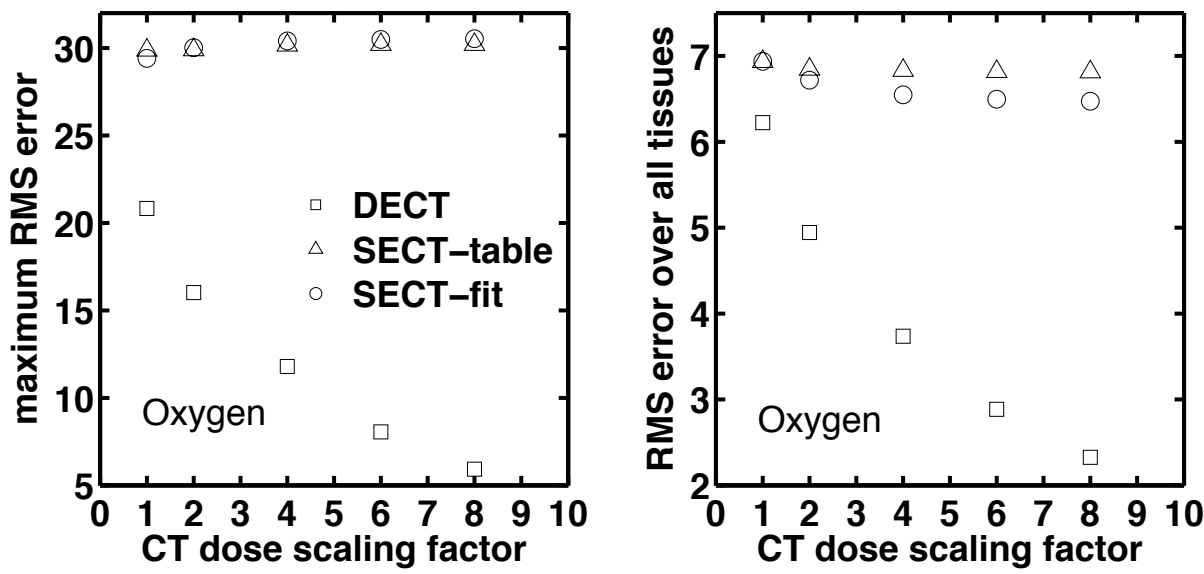

Figure 9.14: Left The maximum RMS error on \%weight $O$ assignment for DECT and SECT as a function of dose level. A CT dose scaling factor of 1 corresponds to a CTDI vol $=20 \mathrm{mGy}$. Right The RMS error on \%weight $O$ for all tissues as a function of dose level. The DECT data is for the abdomen region. The behaviour of the thorax and head regions was similar.

Figures 9.15 and 9.16 show similar data and trends for $\mathrm{H}$ and $\mathrm{N}$. As the \%weight of $\mathrm{H}$ and $\mathrm{N}$ combined is about $10 \%$ for all tissues, the magnitude of absolute errors is lower than for $\mathrm{C}$ and $\mathrm{O}$. DECT provided better results than SECT. 

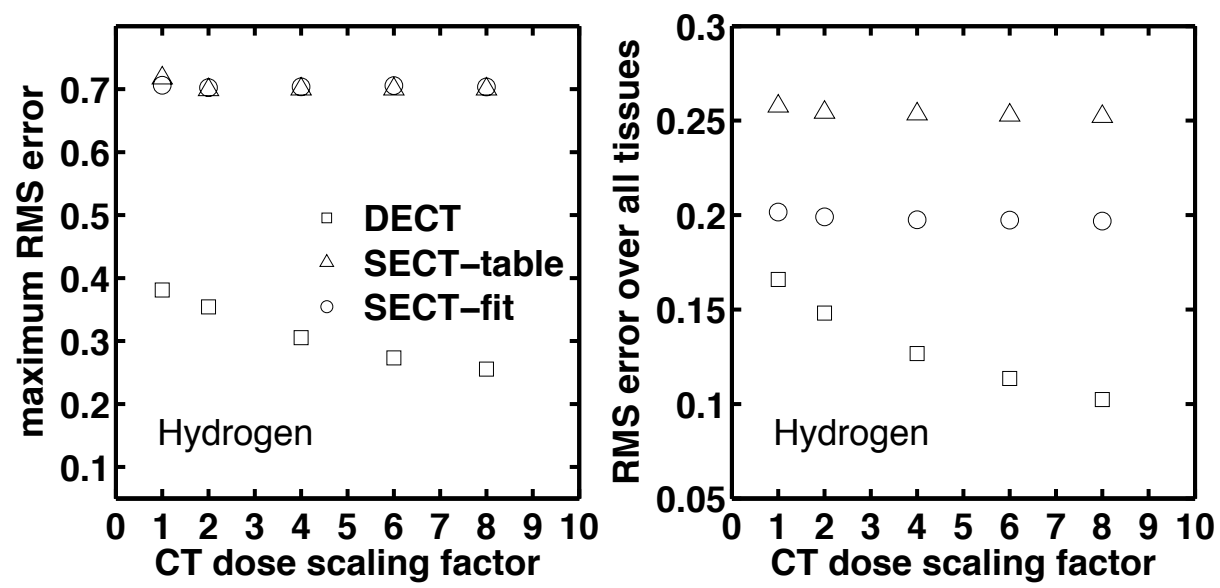

Figure 9.15: Left The maximum RMS error on \%weight $H$ assignment for DECT and SECT as a function of dose level. A CT dose scaling factor of 1 corresponds to a CTDI $\mathrm{vol}=20 \mathrm{mGy}$. Right The $R M S$ error on \%weight $H$ for all tissues as a function of dose level. The DECT data is for the abdomen region. The behaviour of the thorax and head regions was similar.
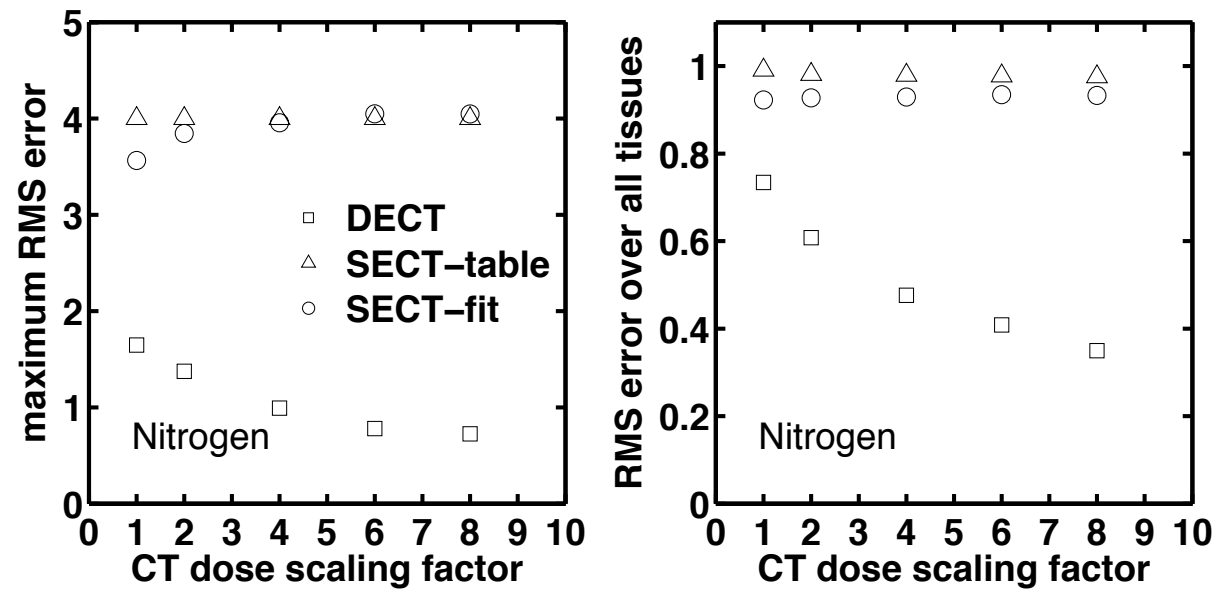

Figure 9.16: Left The maximum RMS error on \%weight $N$ assignment for DECT and SECT as a function of dose level. A CT dose scaling factor of 1 corresponds to a CTDI $\mathrm{vol}=20 \mathrm{mGy}$. Right The $R M S$ error on \%weight $N$ for all tissues as a function of dose level. The DECT data is for the abdomen region. The behaviour of the thorax and head regions was similar.

Our results indicate that DECT provides improved accuracy in \%weight assignment for carbon and oxygen over SECT. The improvement is conditional to reducing the noise levels in DECT images. We used IR which provided a reduction of noise by a factor $\sim 2$ over FBP. Whether increasing imaging dose to further reduce noise levels is acceptable needs to be evaluated in the context of the radiation dose to be delivered during treatment and the added benefits of an improved \%weight carbon and oxygen assignment. Investigating the impact through predicted distributions of beta emitters 
or prompt gamma emitters, based on SECT and DECT imaging is required. AAPM Task Group 75 reports planning CT CTDI ${ }_{\text {vol }}$ ranging from 4 to $116 \mathrm{mGy}$, with average values for the head, chest and abdomen of 79, 10 and 12 respectively (Table VII in that publication $)^{28}$. This range approximately covers the dose levels investigated in this work, except 160 mGy.

Figure 9.17 shows the effect of applying a systematic HU shift on the assignment of \%weight $\mathrm{O}$. We see that beyond a systematic shift of $5 \mathrm{HU}$ the gains observed by performing DECT scanning at low noise levels are lost.

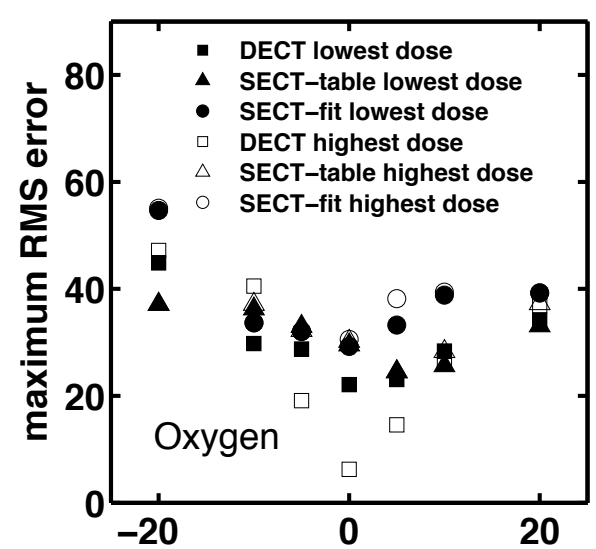

systematic HU shift

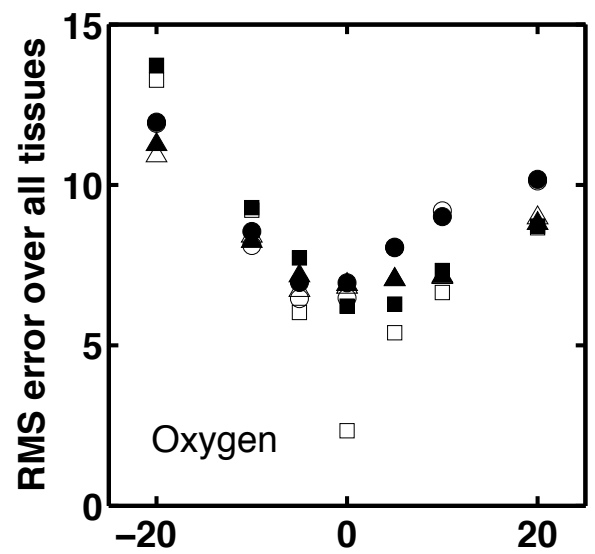

systematic HU shift

Figure 9.17: Left Maximum RMS error on \%weight $O$ assignment for DECT and SECT as a function systematic HU shift. The lowest dose corresponds to $20 \mathrm{mGy}$ and the highest dose to $160 \mathrm{mGy}$. Right RMS error on \%weight $O$ over all tissues. The DECT data is for the abdomen region. The behaviour of the thorax and head regions was similar.

A potential weakness of our study is the reliance on the stoichiometric calibration method to predict the measured $\mathrm{HU}$ of human tissues. The largest difference between the $\mathrm{HU}$ predicted by the stoichiometric procedure and the ones we measured for the Gammex RMI 465 phantom were $60 \mathrm{HU}$ at $80 \mathrm{kVp}$ and $45 \mathrm{HU}$ at $140 \mathrm{kVp}$. It is not straightforward to perform such an estimation for the reference human tissues as they are not readily measured.

The reason we used the stoichiometric procedure to calculate HU was the one-toone relationship shown in Figure 9.5. This let us bypass the calculation of $Z_{\text {eff }}$ from DECT data using knowledge of the DECT scanner spectra ${ }^{17}$, which can be difficult to obtain. With this spectral knowledge $Z_{\text {eff }}$ can be calculated for scanned media without making use of the stoichiometric procedure. Our $\left(Z_{\text {eff }}, \rho_{\mathrm{e}}\right)$ space approach is thus dependent on the accuracy with which DECT scanning can provide estimates of the effective atomic number and electron density. 


\section{CONCLUSION}

In the absence of image noise, DECT provides excellent assignment of \%wC,O when using the $\left(Z_{\text {eff, }}, \rho_{\mathrm{e}}\right)$ space approach (errors $\left.<2 \%\right)$. The $Z_{\text {eff }}$ vs $\% w C, O$ and $\mathrm{HU}$ vs $\% w C, O$ approaches, based on fits of reference human tissue data, have considerable residuals on \%wC,O (up to $15 \%$ and $30 \%$ respectively). The $\left(Z_{\text {eff, }}, \rho_{\mathrm{e}}\right)$ space approach is sensitive to image noise which degrades its accuracy. At $20(80)$ mGy the RMS error over all tissues was 5.8(3.7)\%wC for DECT and 7.5(6.2)\%wC for SECT. At all dose levels investigated in this study, the accuracy of the DECT approach was superior to the SECT approach when evaluated by the RMS error over all tissues and the maximum RMS error. This was also true for \%wH and \%wN assignment. The SECT approach showed little sensitivity to image noise while the DECT approach showed improved accuracy at lower noise levels which correspond to higher imaging doses or improved image reconstruction schemes. However, the DECT method lost its advantage when systematic shifts of more than $\pm 5 \mathrm{HU}$ were applied to the human tissue distributions.

\section{ACKNOWLEDGEMENTS}

The staff of the Maastricht University Medical Center Department of Radiology is gratefully acknowledged for assistance with the operation of the DECT scanner. G.L. acknowledges financial support from the O'Brien Foundation. 


\section{REFERENCES}

${ }^{1}$ U. Schneider, E. Pedroni, and A. Lomax, "The calibration of CT Hounsfield units for radiotherapy treatment planning," Phys Med Biol 41, 111-124 (1996).

${ }^{2}$ M. Kramer, O. Jakel, T. Haberer, G. Kraft, D. Schardt, and U. Weber, "Treatment planning for heavy-ion radiotherapy: physical beam model and dose optimization," Phys Med Biol 45, 3299-3317 (2000).

${ }^{3}$ W. Schneider, T. Bortfeld, and W. Schlegel, "Correlation between CT numbers and tissue parameters needed for Monte Carlo simulations of clinical dose distributions," Phys Med Biol 45, 459-478 (2000).

${ }^{4}$ B. Vanderstraeten, et al., "Conversion of CT numbers into tissue parameters for Monte Carlo dose calculations: a multi-centre study," Phys Med Biol 52, 539-562 (2007).

${ }^{5}$ D.W.O. Rogers, C.M. Ma, B. Waters, G.X. Ding, D. Sheikh-Bagheri, and D. Zhang, BEAMnrc User Manual NRCC Report No PIRS-0509. 2002.

${ }^{6} \mathrm{~F}$. Verhaegen and S. Devic, "Sensitivity study for CT image use in Monte Carlo treatment planning," Phys Med Biol 50, 937-946 (2005).

${ }^{7} \mathrm{~S}$. Espana and H. Paganetti, "The impact of uncertainties in the CT conversion algorithm when predicting proton beam ranges in patients from dose and PET-activity distributions," Phys Med Biol 55, 7557-7571 (2010).

${ }^{8}$ W. Enghardt, P. Crespo, F. Fiedler, R. Hinz, K. Parodi, J. Pawelke, and F. Ponisch, "Charged hadron tumour therapy monitoring by means of PET," Nucl Instrum Meth A 525, 284-288 (2004).

${ }^{9} \mathrm{~K}$. Parodi, et al., "Patient study of in vivo verification of beam delivery and range, using positron emission tomography and computed tomography imaging after proton therapy," Int J Radiat Oncol Biol Phys 68, 920-934 (2007).

${ }^{10}$ C.H. Min, C.H. Kim, M.Y. Youn, and J.W. Kim, "Prompt gamma measurements for locating the dose falloff region in the proton therapy," Appl Phys Lett 89, (2006).

${ }^{11} \mathrm{E}$. Testa, et al., "Monitoring the Bragg peak location of $73 \mathrm{MeV} / \mathrm{u}$ carbon ions by means of prompt gamma-ray measurements," Appl Phys Lett 93, (2008).

${ }^{12} \mathrm{~K}$. Parodi, F. Ponisch, and W. Enghardt, "Experimental study on the feasibility of in-beam PET for accurate monitoring of proton therapy," leee T Nucl Sci 52, 778-786 (2005).

${ }^{13}$ J.C. Polf, S. Peterson, G. Ciangaru, M. Gillin, and S. Beddar, "Prompt gamma-ray emission from biological tissues during proton irradiation: a preliminary study," Phys Med Biol 54, 731-743 (2009).

${ }^{14}$ A. Knopf, K. Parodi, T. Bortfeld, H.A. Shih, and H. Paganetti, "Systematic analysis of biological and physical limitations of proton beam range verification with offline PET/CT scans," Phys Med Biol 54, 4477-4495 (2009).

${ }^{15}$ J. Bauer, D. Unholtz, F. Sommerer, C. Kurz, T. Haberer, J. Debus, and K. Parodi, Experimental validation of simulated positron emitter yields and refined tissue classification for improved Monte Carlo modeling towards offline PET/CT-based verification of proton therapy, in Third European Workshop on Monte Carlo Treatment Planning of the European Workgroup on MCTP. 2012: Sevilla, Spain.

${ }^{16}$ R.A. Rutherford, B.R. Pullan, and I. Isherwood, "Measurement of effective atomic number and electron density using an EMI scanner," Neuroradiology 11, 15-21 (1976).

${ }^{17}$ M. Bazalova, J.F. Carrier, L. Beaulieu, and F. Verhaegen, "Dual-energy CT-based material extraction for tissue segmentation in Monte Carlo dose calculations," Phys Med Biol 53, 2439-2456 (2008).

${ }^{18}$ G. Landry, P.V. Granton, B. Reniers, M.C. Ollers, L. Beaulieu, J.E. Wildberger, and F. Verhaegen, "Simulation study on potential accuracy gains from dual energy CT tissue segmentation for 
low-energy brachytherapy Monte Carlo dose calculations," Physics in medicine and biology 56, 6257-6278 (2011).

${ }^{19}$ M. Yang, G. Virshup, J. Clayton, X.R. Zhu, R. Mohan, and L. Dong, "Theoretical variance analysis of single- and dual-energy computed tomography methods for calculating proton stopping power ratios of biological tissues," Phys Med Biol 55, 1343-1362 (2010).

${ }^{20} \mathrm{~K}$. Parodi, Monte Carlo calculations for PET-based treatment verification of ion beam therapy, in Monte Carlo Techniques in Radiation Therapy. 2013, Taylor Francis: Boca Raton London New York.

${ }^{21}$ H.Q. Woodard and D.R. White, "The composition of body tissues," Br J Radiol 59, 1209-1218 (1986).

${ }^{22}$ D.R. White, H.Q. Woodard, and S.M. Hammond, "Average soft-tissue and bone models for use in radiation dosimetry," Br J Radiol 60, 907-913 (1987).

${ }^{23}$ H.E. Johns and J.R. Cunningham, The physics of radiology. 4th ed. 1983, Springfield, III., U.S.A.: Charles C. Thomas. xix, 796 p.

${ }^{24}$ G. Landry, B. Reniers, P.V. Granton, B. van Rooijen, L. Beaulieu, J.E. Wildberger, and F. Verhaegen, "Extracting atomic numbers and electron densities from a dual source dual energy CT scanner: Experiments and a simulation model," Radiotherapy and Oncology 100, 375-379 (2011).

${ }^{25} \mathrm{M}$. Saito, "Potential of dual-energy subtraction for converting CT numbers to electron density based on a single linear relationship," Med Phys 39, 2021-2030 (2012).

${ }^{26}$ K. Parodi, A. Ferrari, F. Sommerer, and H. Paganetti, "Clinical CT-based calculations of dose and positron emitter distributions in proton therapy using the FLUKA Monte Carlo code," Phys Med Biol 52, 3369-3387 (2007).

${ }^{27}$ M. Yang, Dual Energy Computed Tomography for Proton Therapy Treatment Planning, in Graduate School of Biomedical Sciences. 2011, The University of Texas: Houston.

${ }^{28} \mathrm{M} . J$. Murphy, et al., "The management of imaging dose during image-guided radiotherapy: report of the AAPM Task Group 75," Med Phys 34, 4041-4063 (2007). 



\section{CHAPTER}
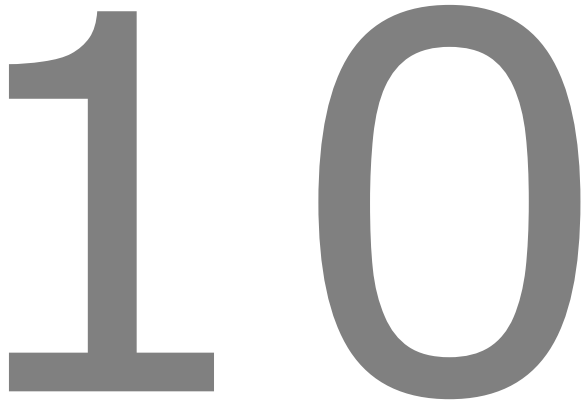

General discussion 



\section{GENERAL DISCUSSION}

A large body of literature, reviewed in Chapter 2 and with contributions from this thesis, now supports the practice of accounting for heterogeneities in brachytherapy. This is endorsed by the recommendations of the recently released report of the AAPM TG-186 which recommends the use of Monte Carlo techniques or equivalent dose calculation methods when computing the radiation dose delivered to patients during the course of brachytherapy treatments.

The patient specific information required to achieve this goal is currently obtained from CT imaging as recommended by TG-186. The standard practice of assigning population averaged tissue composition from $\mathrm{HU}$ can introduce significant uncertainties. This was shown in Chapter 3 where variations in tissue compositions led to significant dose differences when considering brachytherapy based on low energy sources $(<50 \mathrm{keV})$.

The idea of using DECT to refine tissue assignment was first explored by Bazalova et al. for external beam radiotherapy using kilovoltage ( $\mathrm{kV}$ ) and megavoltage (MV) photon beams as well as MV electron beams. DECT showed significant improvement potential for $\mathrm{kV}$ beams where the photoelectric effect plays a more important role than in MV beams. This finding suggested that similar or even greater improvements could be achieved in low-energy brachytherapy where the photoelectric effect plays an even more important role in dose deposition.

We explored the idea in Chapter 5 , using a tissue lookup table based on $Z_{\text {eff }}$ and $\rho_{\mathrm{e}}$ to assign tissue composition based on simulated DECT data for a test geometry containing tissues relevant for brachytherapy. That study showed that DECT provided superior dose calculation accuracy when compared to a conventional SECT approach. We also observed that the noise levels on DECT data can degrade the dose calculation accuracy by reducing the resolving power of the method with respect to identifying tissues based on their $Z_{\text {eff }}$ and $\rho_{\mathrm{e}}$. Initial DECT scans of patients quickly showed that noise levels obtained from standard protocols and reconstruction techniques yielded DECT data of too low quality for use in MC dose calculations.

These findings motivated chapters 7 and 8 which aimed at improving the DECT method. In the case of noise reduction three approaches can be considered: imaging protocol modification such as varying slice thickness or imaging dose, improved image reconstruction and image processing. The approach taken in chapter 7 dealt with improved image reconstruction and afforded a factor 2 reduction of noise levels. Increasing dose levels can also be envisaged in the context of radiotherapy given the high radiation dose delivered during treatment. Chapter 8 made the estimation of $Z_{\text {eff }}$ 
more robust by using a calibration method that obviates the need to have knowledge of the $\mathrm{x}$-ray photon spectrum.

This thesis has aimed at demonstrating that DECT imaging is well understood and can provide the necessary inputs for improved dose calculations in brachytherapy. The question of the optimal use of these inputs in MC applications however remains open. In this thesis we investigated one avenue which consists of using knowledge of reference tissues' $Z_{\text {eff }}$ and $\rho_{\mathrm{e}}$ to compare to estimated $Z_{\text {eff }}$ and $\rho_{\mathrm{e}}$ from DECT scans to assign the properties of the closest reference tissue in the two-dimensional $\left[Z_{\text {eff, }}, \rho_{\mathrm{e}}\right]$ space. This was the method employed in Chapters 5 and 9 . However it is questionable whether reference tissues truly correspond to real human tissues and whether they cover all the possible variations which could be encountered in a patient to patient basis. An alternative would be to directly employ $Z_{\text {eff }}$ and $\rho_{\mathrm{e}}$ to generate a tissue equivalent MC input. $\mathrm{MC}$ requires mass density $\rho$ and elemental composition for each voxel. Density can easily be derived from $\rho_{\mathrm{e}}$ using the relation ${ }^{1}$ :

$\rho=-0.1746+1.176 \rho_{\mathrm{e}}$

which has a maximum error of less than 1\% (does not apply for lung tissues) and is shown in Figure 10.1.

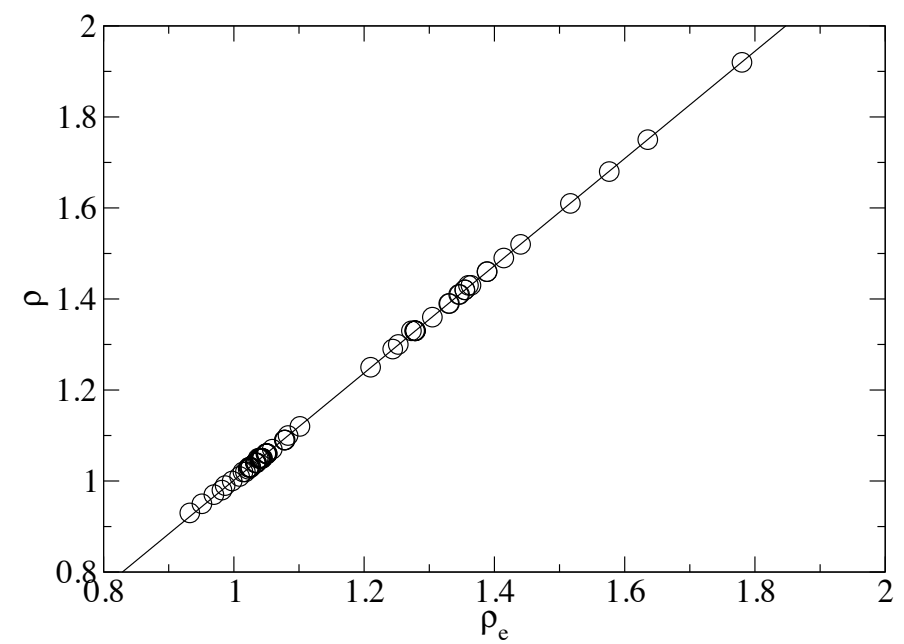

Figure 10.1: Relationship between $\rho$ and $\rho_{e}$ for reference human tissues.

Elemental composition is closely linked with $Z_{\text {eff }}$ and this parameter should be used to derive it. Generally the full composition of human tissue contains a range of elements $(\mathrm{H}, \mathrm{C}, \mathrm{N}, \mathrm{O}, \mathrm{Na}, \mathrm{P}, \mathrm{S}, \mathrm{Cl}, \mathrm{K}, \mathrm{Ca}, \mathrm{Fe})$. Their importance varies with $\mathrm{H}$ and $\mathrm{N}$ adding up to about $10 \%-15 \%, C$ and $O$ to about $80-90 \%$ and the rest present in trace levels of 
a total of about $0.3-1 \%{ }^{2}$. We can use the following relations to derive a $Z_{\text {eff }}$ equivalent composition:

$\sum_{\text {elements }} \omega_{i}=1$

$Z_{\text {eff }}=\sqrt[3.3]{\frac{\sum_{\text {elements }} \omega_{i}\left(Z_{i} / A_{i}\right) Z_{i}^{3.3}}{\sum_{\text {elements }} \omega_{i}\left(Z_{i} / A_{i}\right)}}$

From these two equations we can thus vary the concentrations of two elements to generate a $M C$ tissue substitute with a $Z_{\text {eff }}$ equivalent to that provided by DECT scanning. These two elements could complement a fixed background composition containing $\sim 10 \% \mathrm{H}$ for soft tissues (for these $\omega_{\mathrm{H}} \in[0.09,0.11]$ ). As $\mathrm{N}$ lies between $\mathrm{C}$ and $\mathrm{O}$ and $\omega_{N} \in[0,0.06]$ we can assume that a combination of the two elements $C, O$ should be sufficient. Using $\omega_{\mathrm{H}}=0.1$ and varying $\omega_{\mathrm{C}}$ and $\omega_{\mathrm{O}}$ according to $\omega_{\mathrm{C}}+\omega_{\mathrm{O}}=0.9$ we can thus cover $Z_{\text {eff }} \in[6.75,7.53]$. This range can be extended by allowing a fraction of trace elements to be present in the MC tissue substitute. It is important to note that in this application we do not aim at reproducing a real composition but at obtaining one which yields dose distributions equivalent to that obtained in real tissues with same $Z_{\text {eff. }}$ Figure 10.2 shows radial dose distributions from an ${ }^{125}$ I source in reference muscle tissue and in $\mathrm{MC}$ tissue substitutes generated with various amounts of baseline $\mathrm{H}$ and reproducing the $Z_{\text {eff }}$ of the reference tissue. The RMSE for each concentration of $\mathrm{H}$ are also presented. While all tissues of relevance should be investigated, these preliminary results indicate that this is a viable approach. While White et al presented data where variations of trace elements can affect low-energy brachytherapy dosimetry by a few percents ${ }^{3}$, it is likely that the trace variations will influence $Z_{\text {eff }}$ as well and be compensated by adjusting the amounts of $C$ and $O$. 

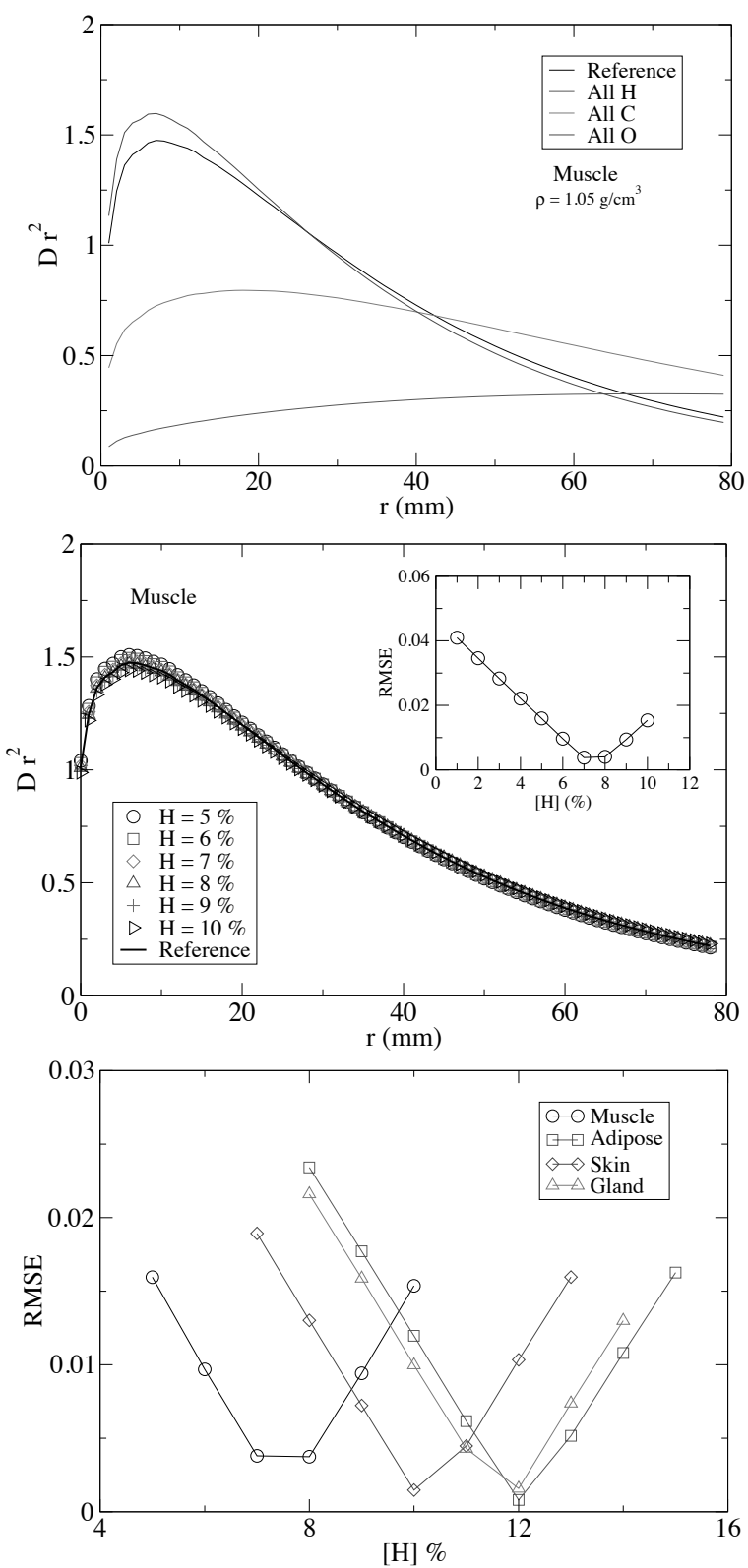

Figure 10.2: Top: Radial dose profile $\mathrm{Dr}^{2}$ from a point source with the inverse square dependence factored out for reference muscle tissue as well as for tissues composed of $100 \% \mathrm{H}, \mathrm{C}$ and $\mathrm{O}$. Middle: Radial dose profiles for reference muscle tissue compared to profiles obtained using equations 10.2 and 10.3 to generate compositions (with $\mathrm{H}, \mathrm{C}$ and $\mathrm{O}$ only) with different levels of $\mathrm{H}$. Bottom: RMSE as a function of $\% \mathrm{H}$ for some reference tissues. 
An alternative to the $Z_{\text {eff }}$ and $\rho_{\mathrm{e}}$ approach is the basis vector approach where measured high and low kVp attenuation coefficients are considered the sum of attenuation coefficients of basis materials. Malusek et al. performed a theoretical investigation of this approach for brachytherapy using 2 and 3 basis materials such as water, lipid and protein ${ }^{4}$. They found relative differences of at most $2 \%$ for mass attenuation and mass energy absorption coefficients at energies above $10 \mathrm{keV}$.

These methods should be compared in terms of accuracy. Of interest would be an experimental validation based on the tissue substitutes of the Gammex RMI 467 phantom. Radiation dose from a brachytherapy source could be measured using radiochromic film or TLDs through a controlled thickness of tissue mimicking material. It may be necessary to shorten the inserts as their length is $7 \mathrm{~cm}$. The inserts would be scanned using DECT to provide voxel estimates of $Z_{\text {eff }}$ and $\rho_{\mathrm{e}}$. The methods described above would then be used to assign compositions and densities to a MC dose calculation algorithm with the aim of reproducing the measured dose distributions.

Chapter 9 explored the use of DECT for assigning the concentrations of $\mathrm{C}$ and $\mathrm{O}$ accurately for particle therapy applications. This is an application where knowledge of the amounts of $\mathrm{C}$ and $\mathrm{O}$ can be useful for comparison of predicted vs. measured positron emitter or prompt gamma distributions. A more broad application in proton therapy is the determination of stopping power ratio (SPR) maps employed in dose calculation and treatment planning. Proton SPR is calculated by:

$$
S P R=\rho_{\mathrm{e}} \frac{\ln \left[2 m_{e} c^{2} \beta^{2} /\left(1-\beta^{2}\right)\right]-\beta^{2}-\ln /}{\ln \left[2 m_{e} c^{2} \beta^{2} /\left(1-\beta^{2}\right)\right]-\beta^{2}-\ln I_{\text {water }}}
$$

where $m_{\mathrm{e}}$ is the electron mass, $c \beta$ is the velocity of the proton and $I$ and $I_{\text {water }}$ are the mean ionization potentials of the material and water respectively. SPR is currently derived from SECT clinically but Yang et al. showed, with a theoretical study, that DECT is superior, in terms of accuracy, than SECT to assign stopping power values ${ }^{5}$. They presented a piecewise linear relationship between $I$ and $Z_{\text {eff }}$ which allowed to use DECT output $\left(Z_{\text {eff }}\right.$ and $\left.\rho_{\mathrm{e}}\right)$ to insert in equation 10.XX. Hunemohr et al. performed an experimental study where the water equivalent path length (WEPL) was calculated for the inserts of the Gammex RMI 467 phantom used in Chapter 8 based on SECT and DECT measurements. They found that the average WEPL residuals for tissue substitutes were $-1.0 \pm 1.8 \%$ for SECT compared to $0.1 \pm 0.7 \%$ for DECT. PMMA, a non tissue substitute, had residuals of $-7.8 \%$ with SECT and $-1.0 \%$ with DECT. Our own measurements (see Chapter 8) showed similar trends when comparing stopping power ratio (SPR) (related to WEPL). We performed two DECT analyses, one using the spectral method and one using the approach of Saito et al. to derive $\rho_{\mathrm{e}}$ and the method of chapter 8 to derive $Z_{\text {eff. }}$ This later method uses the data from the Gammex RMI 467 scans to derive parameters to convert the high and low $\mathrm{kVp} \mathrm{HU}$ to $Z_{\text {eff }}$ and $\rho_{\mathrm{e}}$. The SECT data was 
converted to SPR by fitting two linear SPR vs. HU segments (standard proton therapy procedure). Figure 10.3 confirms that the DECT method is more accurate than the SECT approach.
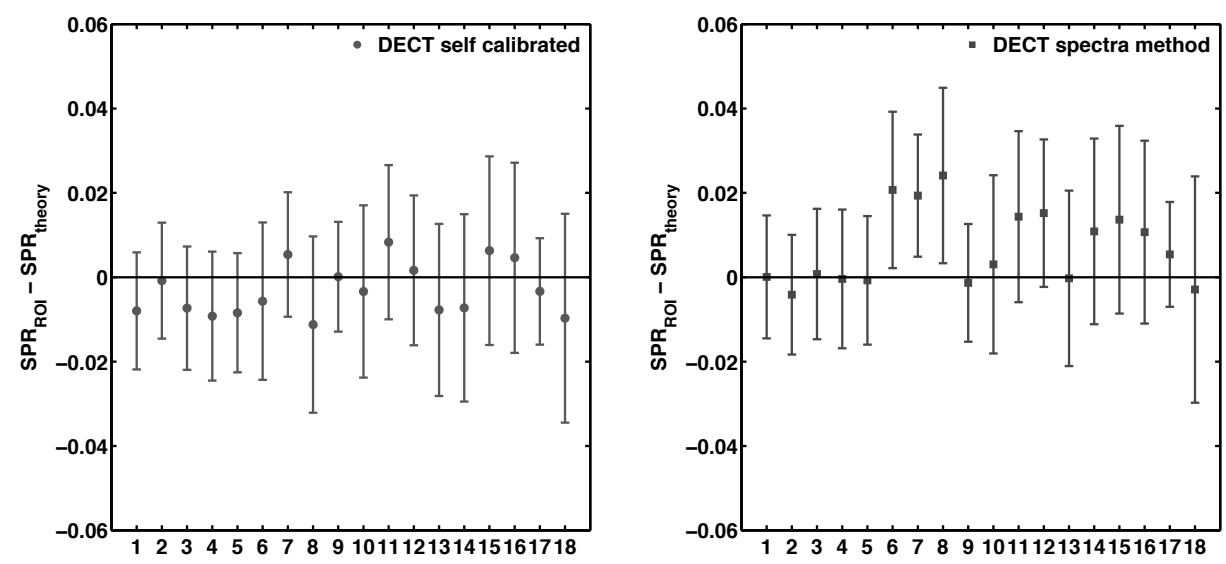

$\begin{array}{cl}1 & \text { solid water } \\ 2 & \text { LN-300 Lung } \\ 3 & \text { solid water } \\ 4 & \text { LV1 Liver } \\ 5 & \text { solid water } \\ 6 & \text { CB2-50\% CaCO3 } \\ 7 & \text { B200 Bone Mineral } \\ 8 & \text { SB3 Cortical Bone } \\ 9 & \text { LN-450 Lung } \\ 10 & \text { BRN-SR2 Brain } \\ 11 & \text { AP6 Adipose } \\ 12 & \text { IB Inner bone } \\ 13 & \text { solid water } \\ 14 & \text { CB-250\% CaCO3 } \\ 15 & \text { Water insert } \\ 16 & \text { BR-12 Breast } \\ 17 & \text { solid water } \\ 18 & \text { solid water }\end{array}$

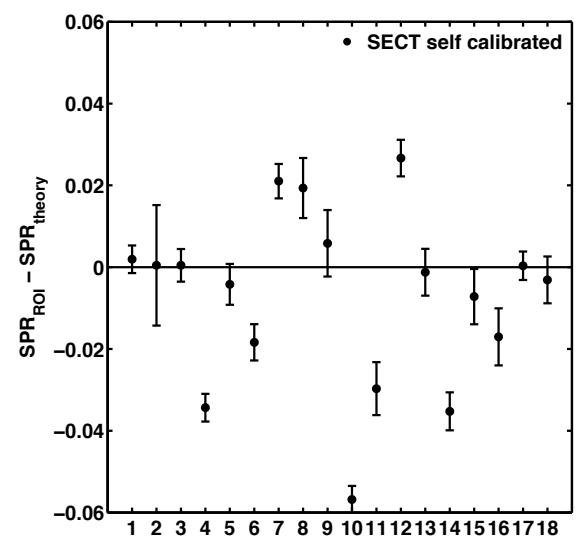

Figure 10.3: SPR differences (measurement - reference) for inserts of the Gammex RMI 467 phantom. Symbols indicate mean differences in a region of interest (ROI) while error bars represent one standard deviation. Left SPR derived from DECT using the method of Saito et al. and Chapter 8. Centre Using the spectral method. Right From SECT using two linear segments to convert HU to SPR.

DECT thus shows promise of improving accuracy in selected radiotherapy applications where sensitivity to tissue composition is important. 
DECT applications such as the derivation of concentrations of $\mathrm{C}$ and $\mathrm{O}$ presented in Chapter 9 rely on prior tissue composition knowledge. Indeed it is not possible to derive the concentrations of all elements present in human tissues with two measurements. Thus the accuracy of this prior knowledge is quite essential. Current reference tissue composition dates from the 1970s and its validation and extension by a measurement campaign where the composition of tissues are measured for several individuals with techniques such as mass spectrometry would be beneficial. For example, a better understanding of the variation of the composition of prostate tissue across individuals and between healthy and cancerous tissue would help understanding what precision and accuracy target should be set for imaging.

Alternatively the use of spectral CT where attenuation can be measured at more than two energies could also yield more information on tissue composition than DECT. Bornefalk showed that the intrinsic dimensionality of low-Z (soft tissues) linear attenuation coefficients is $4^{6}$. Thus it would be reasonable to expect that spectral CT could yield the concentrations of 4 elements.

\section{REFERENCES}

${ }^{1}$ L. Beaulieu, et al., "Report of the Task Group 186 on model-based dose calculation methods in brachytherapy beyond the TG-43 formalism: Current status and recommendations for clinical implementation," Med Phys 39, 6208-6236 (2012).

${ }^{2}$ H.Q. Woodard and D.R. White, "The composition of body tissues," Br J Radiol 59, 1209-1218 (1986).

${ }^{3}$ S.A. White, G. Landry, F. van Gils, F. Verhaegen, and B. Reniers, "Influence of trace elements in human tissue in low-energy photon brachytherapy dosimetry," Phys Med Biol 57, 35853596 (2012).

${ }^{4}$ A. Malusek, M. Karlsson, M. Magnusson, and G.A. Carlsson, "The potential of dual-energy computed tomography for quantitative decomposition of soft tissues to water, protein and lipid in brachytherapy," Phys Med Biol 58, 771-785 (2013).

${ }^{5}$ M. Yang, G. Virshup, J. Clayton, X.R. Zhu, R. Mohan, and L. Dong, "Theoretical variance analysis of single- and dual-energy computed tomography methods for calculating proton stopping power ratios of biological tissues," Phys Med Biol 55, 1343-1362 (2010).

${ }^{6} \mathrm{H}$. Bornefalk, "XCOM intrinsic dimensionality for low-Z elements at diagnostic energies," Med Phys 39, 654-657 (2012). 

SUMMARY 



\section{SUMMARY}

Dose distributions from brachytherapy procedures employing low energy photon sources $(<50 \mathrm{keV})$ are significantly modulated by the composition of human tissues due to the photoelectric effect. This fact was well known at the onset of this thesis and motivated an investigation on methods to better assess the composition of tissues. During this thesis the AAPM Task Group 186 published its report recommending the use of model based dose calculation methods (such as the Monte Carlo simulations heavily used in this thesis) capable of accounting for variations of tissue composition to determine brachytherapy dose distributions, supporting the ideas presented in this thesis. Some of the work of this thesis was cited and included in the report.

This thesis explored two main research lines: the use of Monte Carlo methods in brachytherapy dose calculations to assess their sensitivity with respect to tissue composition variations and the role of dual energy CT (DECT) in the estimation of three dimensional low energy photon cross section maps of patients by identifying tissue types.

A general introduction explaining the need to account for tissue heterogeneities is presented in Chapter 1 along with the objectives of this thesis and an outline of it. The use of Monte Carlo methods in brachytherapy is reviewed in Chapter 2. In that chapter it is shown how the use of Monte Carlo methods evolved in the field of brachytherapy, starting from the calculation of dose distributions around a single brachytherapy source to advanced dose calculation platforms designed for dose calculation of brachytherapy treatments. The chapter also explains why it is not desirable to use water based kernels for low energy brachytherapy dose calculations (as recommended by the now superseded AAPM Task Group report 43). Also covered is the standard use of single energy CT (SECT) to assign to density and tissue composition.

Chapter 3 makes use of tabulated tissue composition to assess the impact of population variations in composition on brachytherapy dose distributions using low energy sources. For several tissues it is possible to find in the literature average tissue composition as well as compositions considered \pm one standard deviation away from the mean. Dose distributions in uniform tissues were calculated to evaluate the impact of these composition variations. A similar procedure was repeated for prostate and breast brachytherapy cases. Effects ranging from $4 \%$ to $30 \%$ were observed indicating that using mean tissue compositions may not be sufficiently accurate.

In Chapter 4 a similar dataset was used to investigate the difference between scoring dose from photons transported in human tissue and depositing their energy in either that tissue or in a reference material which has historically been water. Both quantities can be reported by model based dose calculation algorithms. Furthermore it has not been definitively decided which quantity should be reported. Indeed TG-186 recommends reporting both quantities. Because of the behavior of mass energy absorption coefficients these two quantities are very different (up to $30 \%$ ) for low 
energy photons. In the chapter it is shown that the conversion from one quantity to the other does not vary significantly with distance from a given source. This fact simplifies the conversion from one quantity to the other.

DECT is first introduced in Chapter $\mathbf{5}$ where it is used to extract the effective atomic number $Z_{\text {eff }}$ and relative electron density $\rho_{\mathrm{e}}$ of tissues from CT images taken at low and high $\mathrm{kVp}$. The simulation study presented made use of a simulation package, ImaSim, developed during the MSc thesis of the candidate and further optimized during the PhD work, to generate $\mathrm{CT}$ images at different $\mathrm{kVp}$ settings of virtual calibration phantoms and tissue phantoms. In that chapter a SECT-based method of tissue assignement is compared to a method based on DECT using a $Z_{\text {eff }}$ and $\rho_{\mathrm{e}}$ lookup table. Dose calculations using low energy sources are used as the metric to assess whether tissue assignment is successful. DECT was found to be superior to SECT, although adding noise on the CT images used for DECT analysis decreased the accuracy benefits. This is mostly due to the high noise levels found in $Z_{\text {eff }}$ images which are proportional to the ratio of $\mathrm{CT}$ images.

In Chapter 6 measurements taken at a clinical, dual source DECT scanner are compared to ImaSim simulations. That chapter serves as a validation of the methods presented in Chapter 5. A calibration phantom was thus scanned at the DECT scanner and simulated in ImaSim where models of the CT scanner spectra and detector response were employed. These models were validated by half value measurements at the scanner. Agreement within 5\% was obtained between ImaSim and measurements, validating the methods of Chapter 5.

The next two chapters are dedicated to improving the DECT method to resolve some of the issues observed in chapters 5 and 6 , namely the underestimation of $Z_{\text {eff }}$ for dense materials and the high noise levels on $Z_{\text {eff }}$ images. Chapter 7 presents a method for estimating $Z_{\text {eff }}$ from a pair of high and low $k V p$ CT images based on a calibration procedure using a $\rho_{\mathrm{e}}$ calibration phantom frequently used in radiation therapy quality assurance. A parameterization of the ratio of high and low attenuation coefficients vs. $Z_{\text {eff }}$ is used to fit the measured data. The fit parameters can subsequently be used to convert any measurement to $Z_{\text {eff. }}$ The method is simpler than the method employed in chapters 5 and 6 and provides more accurate values for dense materials.

Chapter 8 presents the results of the evaluation of a commercial iterative image reconstruction method. Phantom scans were used to assess the noise reduction afforded by the algorithm. It was found that for a given imaging dose level the reconstruction method could reduce the standard deviation of $Z_{\text {eff }}$ by a factor two. Combining this method with an increase of imaging dose (which may be justifiable in the case of radiotherapy patients who will receive a much higher curative dose) can bring $Z_{\text {eff }}$ noise levels to an acceptable level of around 0.2 units of $Z_{\text {eff. }}$.

The focus on dose distributions is relaxed in Chapter 9 where an attempt was made to estimate concentrations of carbon and oxygen in human tissues using DECT methods. This application is related to particle therapy where there is potention to estimate the range of protons or carbon ions during delivery using secondary particles emitted following nuclear reactions in the body. The two main avenues of research are the detection of annihilation photons following the emission of positrons using PET cameras and the detection of prompt gammas resulting from the relaxation of excited 
nuclei. As measured distributions are generally compared to predictions and production rates are dependent on the amounts of specific elements (namely $C$ and $O$ ) present in the body, there is an interest in accurately estimating the concentration of $\mathrm{C}$ and $\mathrm{O}$. Using the lookup table technique of Chapter 5 DECT-derived $Z_{\text {eff }}$ and $\rho_{\mathrm{e}}$ are converted into a tissue type whose composition is used to assign concentrations of $C$ and $\mathrm{O}$. The method was found to be superior to the state of the art SECT-based stoichiometric calibration procedure.

Finally Chapter 10 gives a general discussion of the use of DECT in brachytherapy and proposes methods for clinical implementation. Besides the lookup table approach of Chapter 5 a MC tissue substitute method based on $Z_{\text {eff }}$ is also suggested. Future perspectives in the field of particle therapy, namely estimation of stopping power ratio for dose calculation, are also discussed. 



\section{LIST OF PUBLICATIONS}





\section{PUBLICATIONS}

\section{Published articles}

T. Benmessaoud, G. Landry, J Gariepy, B. Ramamoorthy, P. Ashrit, A. Hache. High contrast optical switching in vanadium dioxide thin films. Optics Communications (2008) vol. 281 (24) pp. 6024-6027

G. Poludniowski, G. Landry, F. DeBlois, P.M. Evans, F. Verhaegen. SpekCalc: a program to calculate photon spectra from tungsten anode $x$-ray tubes. Physics in medicine and biology (2009) vol. 54 (19) pp. N433-8

G. Landry, B. Reniers, L. Murrer, L. Lutgens, E.B. Gurp, J.P. Pignol, B. Keller, L. Beaulieu, F. Verhaegen. Sensitivity of low energy brachytherapy Monte Carlo dose calculations to uncertainties in human tissue composition. Medical physics (2010) vol. 37 (10) pp. 5188-98

G. Landry, B. Reniers, J.P. Pignol, L. Beaulieu, F. Verhaegen. The difference of scoring dose to water or tissues in Monte Carlo dose calculations for low energy brachytherapy photon sources. Med Phys (2011) vol. 38 (3) pp. 1526-33

G. Landry, B. Reniers, P.V. Granton, B. van Rooijen, L. Beaulieu, J.E. Wildberger, F. Verhaegen. Extracting atomic numbers and electron densities from a dual source dual energy CT scanner: experiments and a simulation model. Radiotherapy and oncology : journal of the European Society for Therapeutic Radiology and Oncology (2011) vol. 100 (3) pp. $375-9$

G. Landry, P. Granton, B. Reniers, M.C. Ollers, L. Beaulieu, J.E. Wildberger, F. Verhaegen. Simulation study on potential accuracy gains from dual energy CT tissue segmentation for low-energy brachytherapy Monte Carlo dose calculations. Physics in medicine and biology (2011) vol. 56 pp. 6257-78

H. Afsharpour, G. Landry, B. Reniers, J.P. Pignol, L. Beaulieu, F. Verhaegen. Tissue modeling schemes in low energy breast brachytherapy. Physics in medicine and biology (2011) vol. 56 (22) pp. 7045-7060

H. Afsharpour, B. Reniers, G. Landry, J.P. Pignol, B.M. Keller, F. Verhaegen, L. Beaulieu. Consequences of dose heterogeneity on the biological efficiency of (103)Pd permanent breast seed implants. Physics in medicine and biology (2012) vol. 57 (3) pp. 809-23

G. Landry, B. Reniers, L. Lutgens, L. Murrer, H. Afsharpour, D. De Haas-Kock, P. Visser, F. Van Gils, F. Verhaegen. Dose reduction in LDR brachytherapy by implanted prostate gold fiducial markers. Medical physics (2012) vol. 39 (3) pp. 1410-7 
B. Reniers, G. Landry, R. Eichner, A. Hallil, F. Verhaegen. In vivo dosimetry for gynaecological brachytherapy using a novel position sensitive radiation detector: Feasibility study. Medical physics (2012) vol. 39 (4) pp. 1925-35

H. Afsharpour, G. Landry, M. D’Amours, S. Enger, B. Reniers, E. Poon, J.F. Carrier, F. Verhaegen, L. Beaulieu. ALGEBRA: ALgorithm for the heterogeneous dosimetry based on GEANT4 for BRAchytherapy. Physics in medicine and biology (2012) vol. 57 (11) pp. $3273-80$

S White, G. Landry, F. Van Gils, F. Verhaegen, B. Reniers. Influence of trace elements in human tissue in low-energy photon brachytherapy dosimetry. Physics in medicine and biology (2012) vol. 57 (11) pp. 3585-96

S. J. van Hoof, P.V. Granton, G. Landry, M. Podesta, F. Verhaegen. Evaluation of a novel triple-channel radiochromic film analysis procedure using EBT2. Physics in medicine and biology (2012) vol. 57 (13) pp. 4353-4368

P.V. Granton, M. Podesta, G. Landry, S. Nijsten, G. Bootsma, F. Verhaegen. A combined dose calculation and verification method for a small animal precision irradiator based on onboard imaging. Medical physics (2012) vol. 39 (7) pp. 4155

${ }^{1}$ S. Enger, ${ }^{*}$ G. Landry, M. D'Amours, F. Verhaegen, L. Beaulieu, M. Asai, J. Perl. Layered Mass Geometry: a novel technique to overlay seed or applicator onto patient geometry in Geant4 brachytherapy simulations. Physics in medicine and biology (2012) vol. 57 (19) pp. 6269-77

G. Landry, K. Parodi, J.E. Wildberger, F. Verhaegen. Deriving concentrations of oxygen and carbon in human tissues using single- and dual-energy CT for ion therapy applications. Physics in medicine and biology (2013) vol. 58 (15) pp. 5029-5048

G. Landry, J. Seco, M. Gaudreault, F. Verhaegen. Deriving effective atomic numbers from DECT based on a parameterization of the ratio of high and low linear attenuation coefficients. Physics in medicine and biology (2013) vol. 58 (19) pp. 6851-6866

\section{Book chapters}

G. Landry, M.R. Rivard, J.F. Williamson, F. Verhaegen, Monte Carlo methods and applications for brachytherapy dosimetry and treatment planning. In "Monte Carlo Techniques in Radiotherapy", Editors Joao Seco and Frank Verhaegen, Taylor \& Francis, 2013.

\footnotetext{
${ }^{1}$ Authors contributed equally
} 


\section{Published abstracts}

G. Poludniowski, F. deBlois, G. Landry, P. Evans, F. Verhaegen. SpekCalc: a free and user-friendly software program for calculating x-ray tube spectra. $51^{\text {st }}$ Meeting of the American Association of Physicists in Medicine (AAPM), Anaheim, California, July 2630, 2009.

M. Bazalova, G. Landry, Beaulieu L, Reniers B, Verhaegen F. An algorithm for metal streaking artifact reduction in cone beam CT. $51^{\text {st }}$ Meeting of the American Association of Physicists in Medicine (AAPM), Anaheim, California, July 26-30, 2009.

G. Landry, F. deBlois, F. Verhaegen. ImaSim, an animated tool for teaching imaging. $51^{\text {st }}$ Meeting of the American Association of Physicists in Medicine (AAPM), Anaheim, California, July 26-30, 2009.

M. Bazalova, G. Landry, L. Beaulieu, F. Verhaegen. An algorithm for metal streaking artifact reduction in cone beam CT for brachytherapy. $10^{\text {th }}$ Biennial ESTRO Physics Meeting, Maastricht, Aug 30-Sept 3, 2009.

G. Landry, F. DeBlois, M. Bazalova, F. Verhaegen. Teaching imaging made easy: the interactive software tool ImaSim. $10^{\text {th }}$ Biennial ESTRO Physics Meeting, Maastricht, Aug 30-Sept 3, 2009.

G. Poludniowski, G. Landry, F, deBlois, P. Evans, F. Verhaegen. The SpekCalc utility: educational software for modelling x-ray tube spectra in the range $40-300 \mathrm{kV} .10^{\text {th }}$ Biennial ESTRO Physics Meeting, Maastricht, Aug 30-Sept 3, 2009.

E. Bloemen-van Gurp E, J. Wittych, G. Landry, B. Reniers, L. Murrer, F. Verhaegen. 4D in vivo dosimetry in brachytherapy using the RADPOS system: characterization of a positioning system. $29^{\text {th }}$ ESTRO meeting, Barcelona, sept 12-16, 2010.

G. Landry, B. Reniers, B. Keller, E Bloemen van Gurp, L. Murrer, J.P. Pignol, F. Verhaegen. Influence of the compositional uncertainty of human tissues on LDR brachytherapy Monte Carlo dose calculations. $29^{\text {th }}$ ESTRO meeting, Barcelona, sept 12-16, 2010.

H. Afsharpour, G. Landry, B. Reniers, J.P. Pignol, L. Beaulieu, F. Verhaegen. Different tissue modeling schemes in post-implant assessment of breast LDR brachytherapy dosimetry. Annual meeting of the American Brachytherapy Society, San Diego, April 14-16, 2011. 
H. Afsharpour, G. Landry, B. Reniers, J.P. Pignol, L. Beaulieu, F. Verhaegen. Breast tissue modeling in Monte Carlo post-implant evaluation of breast LDR brachytherapy. ESTRO meeting, London, May 8-12, 2011.

B. Reniers B, G. Landry, R. Eichner, E. Bloemen-van Gurp, L. Murrer, F. Verhaegen. In vivo dosimetry for gynaecological brachytherapy based on a novel RADPOS system. ESTRO meeting, London, May 8-12, 2011.

G. Landry, B. Reniers, J.P. Pignol, L. Beaulieu, F. Verhaegen. Dose to medium and waer in low energy brachytherapy. ESTRO meeting, London, May 8-12, 2011.

G. Landry, B. Reniers, P. Granton, F. Verhaegen. On the potential of dual energy CT tissue segmentation in low energy brachytherapy dose calculations. ESTRO meeting, London, May 8-12, 2011.

B. Reniers, G. Landry, R. Eichner, A. Hallil, F. Verhaegen. Use of the novel RadPos system for in-vivo dose verification in gynaecological brachytherapy treatment. AAPM meeting, Vancouver, Jul 31-Aug 4, 2011.

H. Afsharpour, J.F. Carrier, M. D’Amours, S. Enger, J. Perl, M. Asai, G. Landry, F. Verhaegen, L. Beaulieu. G4DBR: A fast Geant4-based Monte Carlo Dosimetry platform for Brachytherapy. AAPM meeting, Vancouver, Jul 31-Aug 4, 2011.

G. Landry, P. Granton, B. Reniers, B. van Rooien, L. Beaulieu, J. Wildberger, F. Verhaegen. Evaluating the impact of dual energy CT on LDR brachytherapy dose calculations. AAPM meeting, Vancouver, Jul 31-Aug 4, 2011.

F. Verhaegen, G. Landry, E. Seravalli. The sensitivity of tissue assignment in Monte Carlo dose calculations. Fourth International Workshop entitled: "International Workshop on Recent Advances in Monte Carlo Techniques for Radiation Therapy, Montreal, June 8-10, 2011.

B. Reniers, G. Landry, R. Eichner, H. Abdelbasset, F. Verhaegen. In-vivo dosimetry for gynaecological brachytherapy using a novel detector system. ICTR conference, Geneva, feb 27-march 22012.

J.E. Wildberger, R.G.H. Beets-Tan, F. Verhaegen, G. Landry, D. de Ruysscher, E. Klotz, M. Das. Dual energy CT: current clinical status. ESTRO meeting, Barcelona, May 9-13, 2012.

S. White, G. Landry, F. van Gils, F. Verhaegen, B. Reniers. Influence of high atomic number elements in human tissue in dosimetry for low energy photon brachytherapy. ESTRO meeting, Barcelona, May 9-13, 2012. 
E. Adolfsson, S. White, G. Landry, G.A. Carlsson, H. Gustafsson, E. Lund, B. Reniers, F. Verhaegen, A. Carlsson Tedgren. Measurements around an electronic brachytherapy source using lithium formate EPR dosimeters and GafChromic film. ESTRO meeting, Barcelona, May 9-13, 2012.

G. Landry, B. Reniers, L. Lutgens, L. Murrer, H. Afsharpour, D. de Haas-Kock, P. Visser, F. van Gils, F. Verhaegen. Fiducial markers in combined EBRT-LDR therapy of prostate cancer: influence on LDR dose. ESTRO meeting, Barcelona, May 9-13, 2012.

B. Reniers, G. Janssens, J. Orban de Xivry, G. Landry, F. Verhaegen. Dose distribution for gynaecological brachytherapy with dose accumulation between insertions: feasibility study. ESTRO meeting, Geneva, April 19-23, 2013.

S. White, G. Landry, R. Holt, B. Neslissen, T. Rusch, L. Beaulieu, F. Verhaegen, B. Reniers. Comparison of TG-43 and TG-186 in breast irradiation using a low energy electronic brachytherapy source. ESTRO meeting, Geneva, April 19-23, 2013.

G. Landry, B. Reniers, E. Vigneault, L. Beaulieu, J. Wildberger, F. Verhaegen. Dual energy CT facilitates LDR brachytherapy seed metal artefact reduction. ESTRO meeting, Geneva, April 19-23, 2013.

S. White, G. Landry, R.W. Holt, T. Rusch, L. Beaulieu, F. Verhaegen, B. Reniers. Dosimetric impact of tissue heterogeneity in low energy accelerated partial breast irradiation: a Monte Carlo study. ABS Annual Meeting, New Orleans, April 18-20, 2013.

G. Landry, K. Parodi, F. Verhaegen. Single and dual energy CT assignment of carbon and oxygen contents of human tissues for ion therapy applications. PTCOG meeting, Essen, June 2-8, 2013. 

ACKNOWLEDGEMENTS 



\section{ACKNOWLEDGEMENTS}

I would first like to acknowledge my promotor and copromotor Prof. Dr. Frank Verhaegen and Prof. Dr. Luc Beaulieu.

Frank, thank you for providing me with the opportunity to pursue graduate studies in Europe. This experience changed my perception of life and opened up the world. Thank you also for teaching me how to be a researcher; from writing papers to preparing conference presentations. I hope we will continue collaborating. Thanks to both you and Brigitte for many pleasant evenings, great food and wine.

Luc, thank you for the many comments and suggestions throughout the years which always improved the manuscripts we collaborated on. These collaborations greatly improved my thesis and were a beneficial experience.

I owe a lot to Dr. Brigitte Reniers who gave me guidance and advice in the first year of my PhD. Your vast expertise enabled me to "hit the ground running" and was a major force in our publications.

I thank Dr. Mark Rivard for guiding me in the process of writing the brachytherapy chapter for Frank's book.

I would like to thank the Brachytherapy Group for supporting my research throughout my stay at Maastro; Lars, Francis, Ludy, Raymond, Danielle, Pieter, Esther thank you. Lars, thank you, as well as Ann, for being great friends at and outside work.

I would like to acknowledge the support of the staff of the Radiology Department led by Prof. Dr. Joachim Wildberger. Access to the dual energy scanner was essential for the completion of this thesis and I am grateful for it.

Thank you Shirin for our collaborations, the LMG paper was quite some fun.

To my colleagues; Skadi, Karen, Sara, Ralph, Stefan, Shane, Davide, Daniela, Erik, Lucas, Davy it was great to work in your company for the last few years. I wish you all the best with your respective projects. Skadi and Geert thanks again for that great sailing trip. Daniela and Anne-Marie thanks for those nice wine-evenings.

Also thanks to my early-days colleagues, Steven, Hugo, Enrica, Miguel and Marco for welcoming me at Maastro. Steven, I am looking forward to more Rotterdam evenings with you and Violet.

Maaike, III miss those mountain bike rides, especially those rare occasions when Wouter and Michel finally showed up. Thanks Wouter for answering my many questions relating to the graduation procedure, as well as our collaboration and some good laughs.

Guys; Mark, Emmanuel, Patrick, Sean, Ruud, Pablo, Gabriel, Mathieu, Leo, Fabien, Raghu - thanks for all the drinks. I cannot say you helped this thesis happen faster, but you definitely made it more fun. Next round is on me.

Mark and Patrick, I greatly appreciate your support as "paranimfen". You both joined the physics research group not long after me and were a source of invaluable feedback. 
Thank you Céline for accompanying me along the way, we shared great experiences. I hope you keep enjoying Europe.

Finally thanks to my parents, Jean-Paul and Violette and my brother Jean-Gabriel for supporting my eandeavours abroad and for never pressuring me to finally return to Canada. Your visits and many video conversations helped reduce the distance. 
CURRICULUM VITAE 



\section{CURRICULUM VITAE}

Born in Caraquet, Canada, Guillaume Landry graduated from the Polyvalente LouisMailloux in 2003 as recipient of the Governor General's Academic Medal. He moved on to study undergraduate physics at the Université de Moncton, developing an interest for research fostered by the National Sciences and Engineering Research Council of Canada (NSERC) Undergraduate Student Research Awards Program. He completed his bachelor studies in 2007, obtaining a second Governor General's Academic Medal. Funded by the New-Brunswick Medical Physics Bursary Program and an NSERC Graduate Scholarship, he was accepted in the Medical Radiation Physics program at McGill University. He studied two years in Montreal, developing software tools for medical imaging research for his Master's thesis. Following a 2009 McGill graduation, he left Canada and joined the research team of the MAASTRO radiation oncology clinic in Maastricht, The Netherlands, as a PhD candidate under supervision of Prof. Dr. Frank Verhaegen, again with NSERC support. His research there touched on topics such as the calculation of radiation dose from radiotherapy and computed tomography imaging. During his MAASTRO period he obtained financial support from the O'Brien Foundation which helped him, along with an ESTRO technology transfer grant and a KWF scholarship, to spend 3 months at the Massachussetts General Hospital in Boston as a visiting scientist to work on proton therapy topics. In 2013 he joined the research group of Prof. Dr. Katia Parodi of the Ludwig Maximilians University in Munich. 
